\title{
ॠUSGS
}

science for a changing world

\section{Digital Database of Microfossil Localities in Alameda and Contra Costa Counties, California}

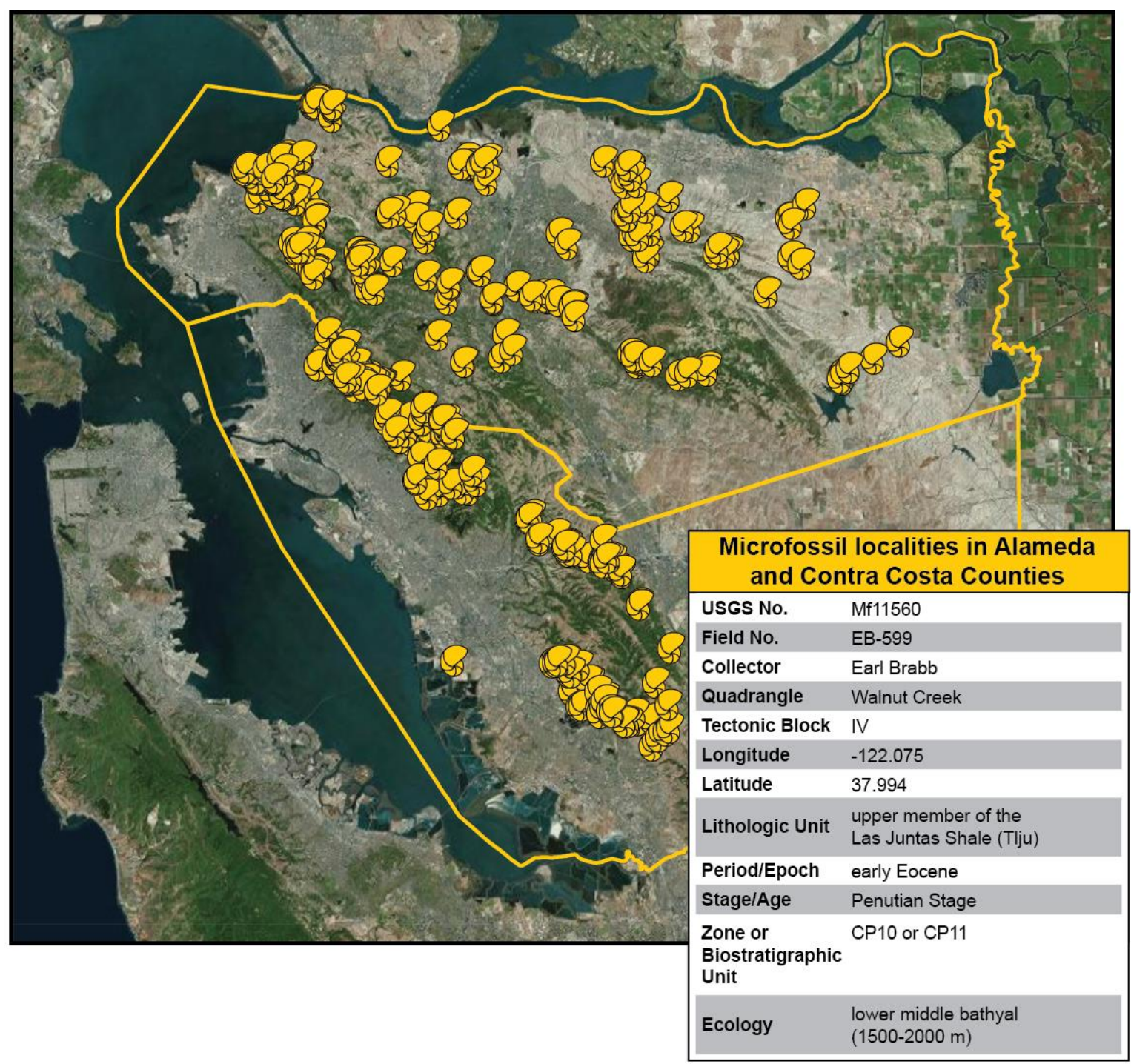

Scientific Investigations Report 2014-5120

U.S. Department of the Interior

U.S. Geological Survey 


\section{COVER}

Image showing microfossil localities in Google Earth with example balloon containing abbreviated sample description. 


\section{Digital Database of Microfossil Localities in Alameda and Contra Costa Counties, California}

By Kristin McDougall and Debra Block

Scientific Investigations Report 2014-5120

U.S. Department of the Interior

U.S. Geological Survey 


\section{U.S. Department of the Interior \\ SALLY JEWELL, Secretary}

\section{U.S. Geological Survey \\ Suzette M. Kimball, Acting Director}

U.S. Geological Survey, Reston, Virginia: 2014

For more information on the USGS-the Federal source for science about the Earth, its natural and living resources, natural hazards, and the environment-visit http://www.usgs.gov or call 1-888-ASK-USGS

For an overview of USGS information products, including maps, imagery, and publications, visit $h$ ttp://www.usgs.gov/pubprod

To order this and other USGS information products, visit http://store.usgs.gov

Suggested citation:

McDougall, Kristin, and Block, Debra, 2014, Digital database of microfossil localities in Alameda and Contra Costa Counties, California: U.S. Geological Survey Scientific Investigations Report 2014-5120, 108 p., http://dx.doi.org/10.3133/sir20145120.

ISSN 2328-0328 (online)

Any use of trade, product, or firm names is for descriptive purposes only and does not imply endorsement by the U.S. Government.

Although this report is in the public domain, permission must be secured from the individual copyright owners to reproduce any copyrighted material contained within this report. 


\section{Contents}

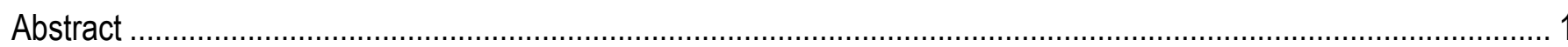

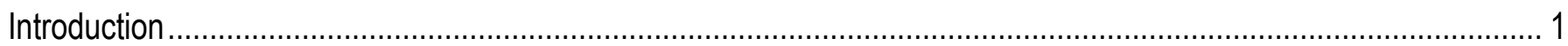

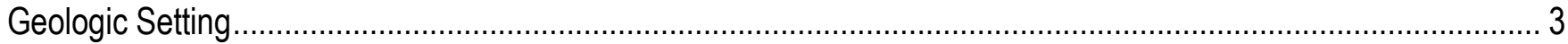

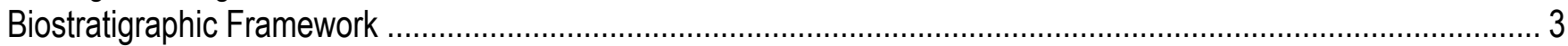

Ecologic Framework

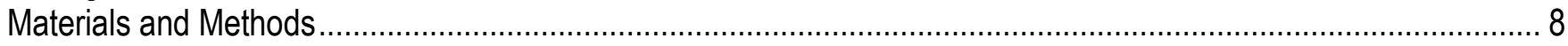

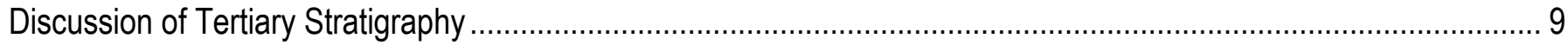

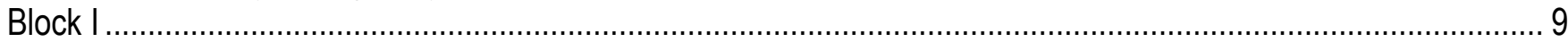

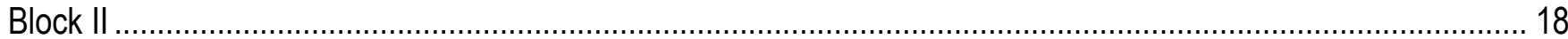

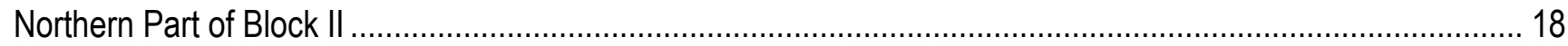

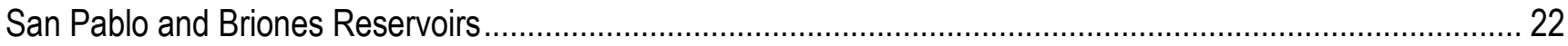

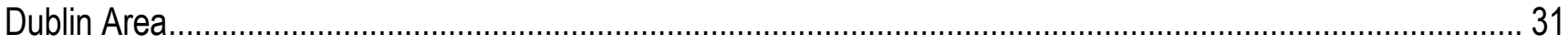

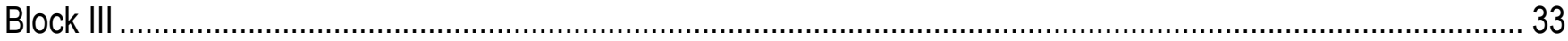

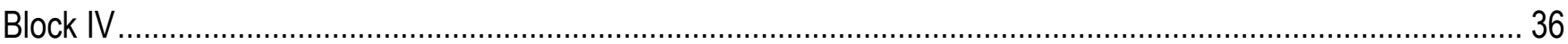

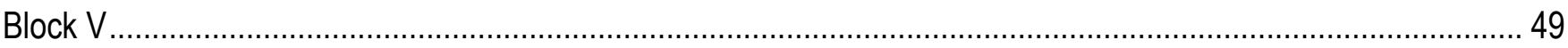

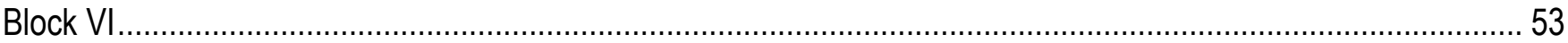

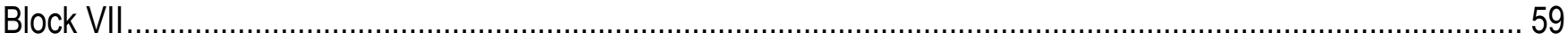

Block IX

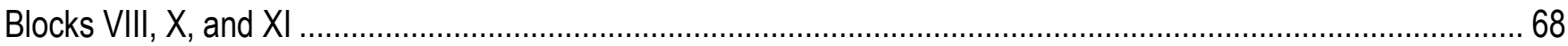

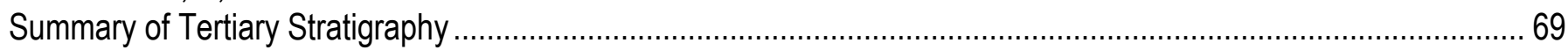

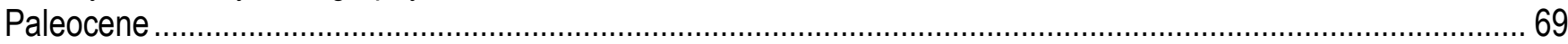

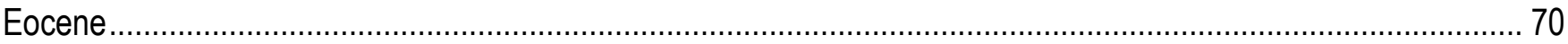

Oligocene

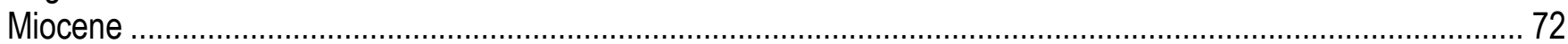

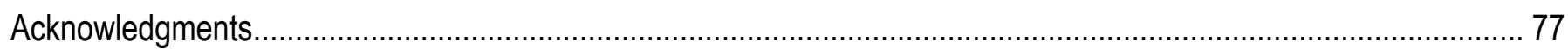

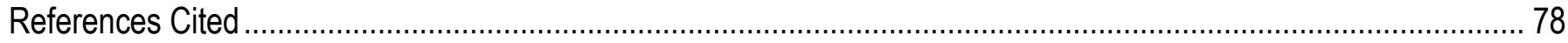

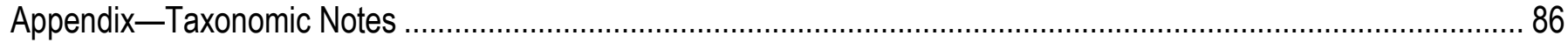

\section{Plate}

Plate 1. Chart showing correlation of stratigraphic units in the tectonic blocks identified in Contra Costa and Alameda Counties. (Available for download at http://pubs.usgs.gov/sir/2014/5120/.)

\section{Figures}

1. Map of the San Francisco Bay area showing the tectonic blocks into which the area is subdivided by faults ........... 4

2. California Cenozoic biostratigraphic framework from McDougall (2008).......................................................... 6

3. Ranges of Paleocene and some Eocene age-diagnostic benthic foraminifers ................................................... 6

4. Ranges of Eocene age-diagnostic benthic foraminifers ............................................................................

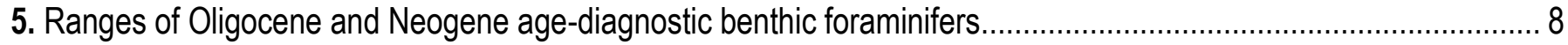

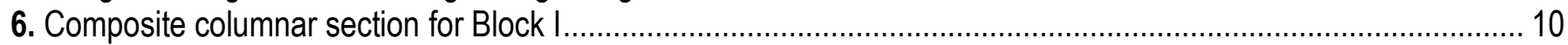

7. Composite columnar section for Block II, northern part and San Pablo and Briones Reservoirs ........................... 19

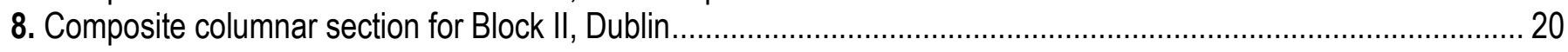

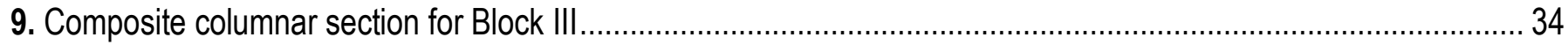

10. Correlation of Pacheco syncline and Selby Point sections ……................................................................. 37 


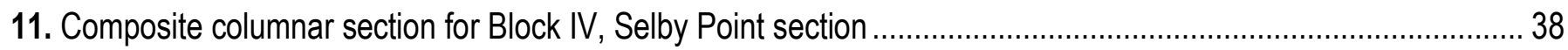

12. Composite columnar section for Block IV, Pacheco syncline..................................................................... 42

13. Composite columnar section for Block V ..................................................................................... 50

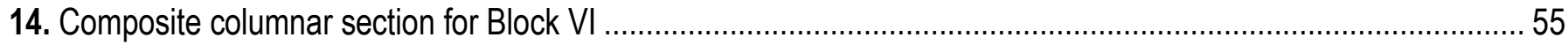

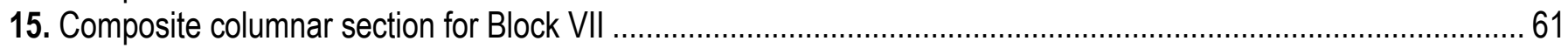

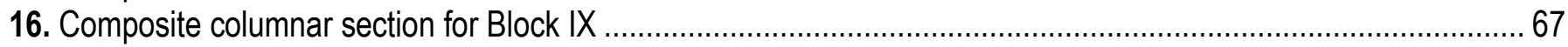

17. Map showing outcrop distribution of Paleocene strata (green) in Alameda and Contra Costa Counties ...................................................................................................................... 70

18. Map showing outcrop distribution of Eocene strata (yellow) in Alameda and

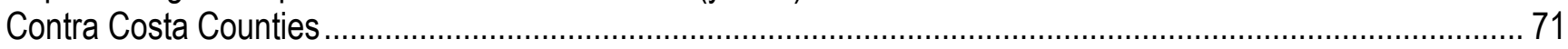

19. Map showing outcrop distribution of Oligocene (pink) and Miocene (teal) strata in Alameda and Contra Costa Counties, not including the Monterey Group ........................................................................ 73

20. Map showing outcrop distribution of units comprising the Miocene Monterey Group in Alameda and Contra Costa Counties

21. Map showing distribution of middle and upper Miocene marine strata (blue areas) in Contra Costa and Alameda Counties, not including the Monterey Group.....

\section{Tables}

1. List of samples without enough information to locate

2. Samples taken from Cretaceous units. .............................................................................................. 2

3. Benthic foraminifers from unit Tes/Tsh unit of Graymer and others (1994a, 1996, 2000) of Block I. ...................... 11

4. Benthic foraminifers from unit Tsm of Graymer and others (1996; Graymer, 2000) of Block I............................. 14

5. Benthic foraminifers from the Claremont chert and rocks mapped as the Tice Shale (Tt), Orinda Formation (Tor), and Moraga Formation (Tms) in Block I.............................................................. 17

6. Benthic foraminifers from unit Tshc of Graymer and others $(1994 a, 1996)$ in the northern part of Block II.

7. Benthic foraminifers from unit Tshc of Graymer and others $(1994 a, 1996)$ near San Pablo Reservoir in Block II.

8. Benthic foraminifers from unit Tshc of Graymer and others (1994a, 1996) near Briones Reservoir

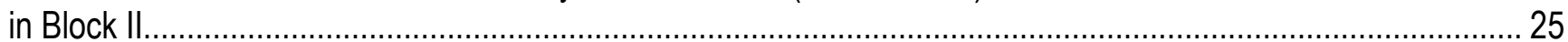

9. Benthic foraminifers from the San Ramon Sandstone (?) in Block II ............................................................. 27

10. Benthic foraminifers from the Neogene Sobrante Sandstone, unit Tus of Graymer and others (1994a, 1996), Claremont Shale, and Oursan Sandstone near San Pablo and Briones Reservoirs in Block II .... 28

11. Benthic foraminifers from the Neogene Tice Shale, Hambre Sandstone and Rodeo Shale near

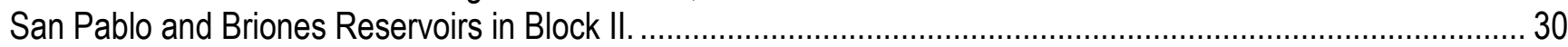

12. Benthic foraminifers from the Neogene units Tus and Tms near San Pablo and Briones Reservoirs in Block II.

13. Benthic foraminifers from Neogene units (Graymer and others, 1994a, 1996) near Dublin in Block II ................. 32

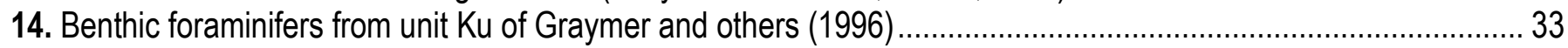

15. Benthic foraminifers in unit Tshc of Graymer and others $(1994 a, 1996)$ in Block III ......................................... 35

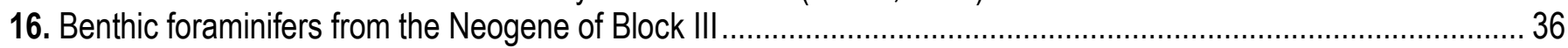

17. Benthic foraminifers from the Vine Hill Sandstone in Block IV .................................................................... 39

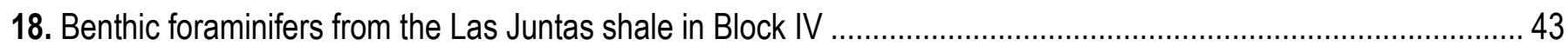

19. Benthic foraminifers from the Muir Sandstone in Block IV ..................................................................... 48

20. Benthic foraminifers from the Domengine Formation and unit Kss of Graymer and others (1994a, 1996) in Block V. 
21. Benthic foraminifers from the Cierbo Sandstone in Block V....................................................................... 53

22. Benthic foraminifers from Paleogene units of Block VI ....................................................................... 56

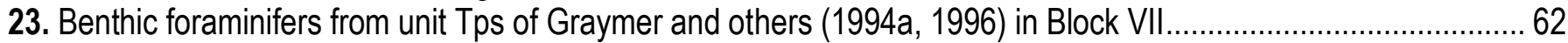

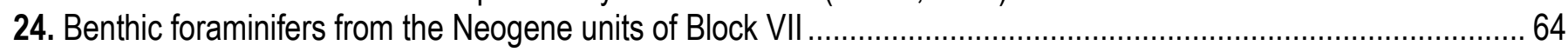

25. Benthic foraminifers from the Neogene Briones Sandstone and Claremont Shale(?) of Block VII......................... 65

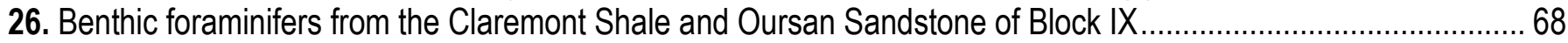


This page left intentionally blank 


\title{
Digital Database of Microfossil Localities in Alameda and Contra Costa Counties, California
}

\author{
By Kristin McDougall and Debra Block
}

\begin{abstract}
The eastern San Francisco Bay region (Contra Costa and Alameda Counties, California) is a geologically complex area divided by faults into a suite of tectonic blocks. Each block contains a unique stratigraphic sequence of Tertiary sediments that in most blocks unconformably overlie Mesozoic sediments. Age and environmental interpretations based on analysis of microfossil assemblages are key factors in interpreting geologic history, structure, and correlation of each block. Much of this data, however, is distributed in unpublished internal reports and memos, and is generally unavailable to the geologic community. In this report the U.S. Geological Survey microfossil data from the Tertiary sediments of Alameda and Contra Costa Counties are analyzed and presented in a digital database, which provides a user-friendly summary of the micropaleontologic data, locality information, and biostratigraphic and ecologic interpretations.
\end{abstract}

\section{Introduction}

The purpose of this paper is to provide age and environmental interpretations of marine strata in Alameda and Contra Costa Counties based on microfossils. The purpose of the associated digital database is to provide a quick and easy means of accessing micropaleontologic data, locality information, and a summary of the interpretations. Data for this study are derived from microfossil samples in the U.S. Geological Survey collections, including new samples collected during field work related to the production of U.S. Geological Survey Open-File Reports and Miscellaneous Field Maps (Blake and others, 2000; Graymer, 2000; Graymer and others, 1994a, b, 1996, 2002a; Helley and Graymer, 1997a, b) and samples that were collected to support earlier mapping efforts (Hill, 1983; Radbruch-Hall, 1957, 1969; Radbruch and Case, 1967). The older samples were reviewed for consistency with modern biostratigraphic understanding and revisions made as appropriate. Locality descriptions of some of the older samples were inadequate and the samples could not be located with any certainty. These samples are excluded from the database (table 1). Although Cretaceous samples are included in the database, they are not discussed in this paper (table 2).

This paper describes the age and ecology of the Tertiary samples and marine units based on microfossils (primarily benthic foraminifers), correlates the various units, and provides a brief geologic history of the eastern San Francisco Bay. We have used an internationally accepted time scale (Gradstein and others, 2004; Gradstein and Ogg, 2005) to which the local California stages and zones are correlated (McDougall, 2008) and an ecological framework that includes local as well as cosmopolitan species. Because the Tertiary section in Alameda and Contra Costa Counties is divided into tectonic blocks (Graymer and others, 1994a, 1996), microfossil assemblages in each block are examined to develop intrablock and regional stratigraphic relationships. The microfossil assemblages 
are also analyzed to determine the ecology of the block through time and develop a means to correlate basins or parts of basins that have been displaced by the faults.

Table 1. List of samples without enough information to locate.

\begin{tabular}{|c|c|c|}
\hline Mf591 & Mf 776 & Mf 2029 \\
\hline Mf 618 & Mf 777 & Mf 2030 \\
\hline Mf 758 & Mf 793 & Mf 2031 \\
\hline Mf 760 & Mf 795 & Mf 2032 \\
\hline Mf 763 & Mf 796 & Mf 2034 \\
\hline Mf 764 & Mf 881 & Mf 2035 \\
\hline Mf 766 & Mf 2025 & Mf 3259 \\
\hline Mf 767 & Mf 2026 & Mf 3260 \\
\hline Mf 769 & Mf 2027 & Mf 8516 \\
\hline Mf 772 & Mf 2028 & \\
\hline
\end{tabular}

Table 2. Samples taken from Cretaceous units.

[Asterisks $(*)$ indicate samples taken from units mapped as Cretaceous that contain a Tertiary microfauna. A solid circle $(\bullet)$ indicates samples that are mapped as a Tertiary unit but contain a Cretaceous microfauna. Many of these assemblages are discussed in this paper]

\begin{tabular}{|c|c|c|c|c|c|c|c|}
\hline Mf522* & Mf613 & Mf797 & Mf7781 & Mf7934 & Mf7955 & Mf8008 & Mf8512 \\
\hline Mf523 & Mf614 & Mf798 & Mf7782 & Mf7935 & Mf7956 & Mf8017 & Mf8514 \\
\hline Mf524 & Mf615 & Mf799 & Mf7783 & Mf7936 & Mf7966* & Mf8122* & Mf8515 \\
\hline Mf525 & Mf616 & Mf804* & Mf7784 & Mf7937 & Mf7985* & Mf8186* & Mf8532 \\
\hline Mf526• & Mf617 & Mf809 & Mf7788 & Mf7938 & Mf7991 & Mf8189 & Mf8533 \\
\hline Mf528 & Mf619 & Mf810 & Mf7789 & Mf7939 & Mf7992 & Mf8195* & Mf8534 \\
\hline Mf529 & Mf620 & Mf811 & Mf7790 & Mf7940 & Mf7993 & Mf8202 & Mf8535 \\
\hline Mf530 & Mf637• & Mf812 & Mf7791 & Mf7941 & Mf7994 & Mf8240 & Mf8536 \\
\hline Mf531 & Mf638• & Mf813 & Mf7792 & Mf7942• & Mf7995 & Mf8242 & Mf8537 \\
\hline Mf534 & Mf759 & Mf880 & Mf7795 & Mf7943 & Mf7996 & Mf8288 & Mf8538 \\
\hline Mf537 & Mf761* & Mf1096 & Mf7796 & Mf7944 & Mf7997 & Mf8289 & Mf8541 \\
\hline Mf538 & Mf762* & Mf1656 & Mf7797 & Mf7945 & Mf7998 & Mf8290 & Mf8605* \\
\hline Mf539• & Mf765* & Mf2047 & Mf7799 & Mf7946 & Mf7999 & Mf8317 & Mf8606 \\
\hline Mf540 & Mf768* & Mf3264* & Mf7800 & Mf7947 & Mf8000 & Mf8318 & Mf8781 \\
\hline Mf541 & Mf770* & Mf3272* & Mf7801 & Mf7948 & Mf8001 & Mf8411 & Mf8785 \\
\hline Mf542 & Mf773* & Mf3335 & Mf7802 & Mf7949 & Mf8002 & Mf8421 & Mf8786 \\
\hline Mf543 & Mf774* & Mf3337* & Mf7803 & Mf7950 & Mf8003 & Mf8427* & Mf8787 \\
\hline Mf544 & Mf775* & MF5007• & Mf7870 & Mf7951 & Mf8004 & Mf8437 & Mf10232 \\
\hline Mf545• & Mf791* & Mf7766* & Mf7886 & Mf7952 & Mf8005 & Mf8459 & Mf10251 \\
\hline Mf590 & Mf792(A1)* & Mf7776• & Mf7932 & Mf7953 & Mf8006 & Mf8462• & Mf11561* \\
\hline Mf592 & Mf792(B1)* & Mf7777 & Mf7933 & Mf7954 & Mf8007 & Mf8463• & \\
\hline Mf612 & Mf794* & Mf7778 & & & & & \\
\hline
\end{tabular}


This report includes a KMZ file for presenting the data digitally. A KMZ file is a compressed archive consisting of a KML file along with supporting files. KML is a file format used to display geographic data in a geospatial data viewer such as Google Earth. Download the KMZ file onto your hard drive, launch Google Earth, and open the database. Hundreds of location placemarks will pop up on the screen. Each placemark, or microfossil icon, marks a sample location on the Earth's surface. When the cursor rolls over an icon, the associated USGS sample number pops up. Clicking on an icon generates a balloon containing an abbreviated description of the associated sample. If more than one sample is geographically colocated, a click will result in a branchlike structure enumerating the USGS sample numbers. An additional click on the sample of interest will engender the description balloon. Alternatively, a double-click on one of the USGS sample numbers listed under the "Microfossil Data" folder will cause Google Earth to fly to that location. The description balloon contains a link to a PDF file with further sample data as well as a link to the written component of this report. Because the microfossil assemblages are analyzed with respect to the fault-bounded tectonic block in which they occur, the folder "Tectonic Blocks" contains a subfolder entitled "Names and Stratigraphic Columns" with related balloons and containing a link to a PDF file of the corresponding stratigraphic column and paleontologic interpretation, where defined. Supplementary information regarding the structure and content of the digital data may be accessed through the metadata link on the KMZ header balloon.

\section{Geologic Setting}

The geologic structure of the eastern San Francisco Bay region is a complex series of folds and faults that divide the region into subterranes or tectonic blocks (fig. 1; Huey, 1948; Robinson, 1956; Hall, 1958; Colburn, 1961; Bailey and others, 1964; Case, 1968; Nielsen and Brabb, 1979; Graham and others, 1984; Jones and Curtis, 1991; Buising and Walker, 1995; Graymer, 2000; Graymer and others, 1994a, b, 1996, 2005). These subdivisions are defined as having unique stratigraphic sequences that result from differences in environment conditions. The various depositional basins or parts of basins are now juxtaposed by offsets along faults attesting to the complex tectonic history of this region (Graymer and others, 1994a, 1996). Graham and others (1984) and Buising and Walker (1995) identified 4 blocks in the San Francisco Bay region whereas the more detailed studies by Graymer and others (1994a, 1996) identified 10 blocks in Alameda and Contra Costa Counties alone. In the blocks defined by Graymer and others (1994a, 1996), the Tertiary strata rest with angular unconformity on two deformed Mesozoic rock complexes: the Franciscan Complex and a complex that includes the Great Valley sequence, volcanic rocks, and an older accreted oceanic crust (Graymer and others, 1994a, 1996). Although the Tertiary section is unique to each block, the similarities between blocks increase in the younger sedimentary units.

\section{Biostratigraphic Framework}

The California Cenozoic biostratigraphic framework (fig. 2) is a composite of the provincial benthic foraminiferal stages of Kleinpell (1938) and Mallory (1959), with modifications as proposed by Almgren and others (1988), McDougall (1980, 1989, 2008), and Blake (1991). Benthic foraminiferal stage names follow the modifications proposed by McDougall $(1980,1988,1989,1993,2008)$ for the Paleocene and Eocene, and by Blake (1991) for the late Neogene. Zonation of the Oligocene and Miocene primarily follows Kleinpell (1938) with modifications suggested by Addicott and others (1980), Finger (1990), and McDougall (1983; unpublished). Correlation of the benthic foraminiferal stages and zones to the international time scale is discussed in McDougall (2008). Where possible, benthic foraminiferal age interpretations are given in terms of coeval planktic foraminiferal zones or 
calcareous nannofossil zones. Ranges of diagnostic benthic foraminifers which have been compiled from these various studies are given in the appendix and summarized in figures 3, 4, and 5 .

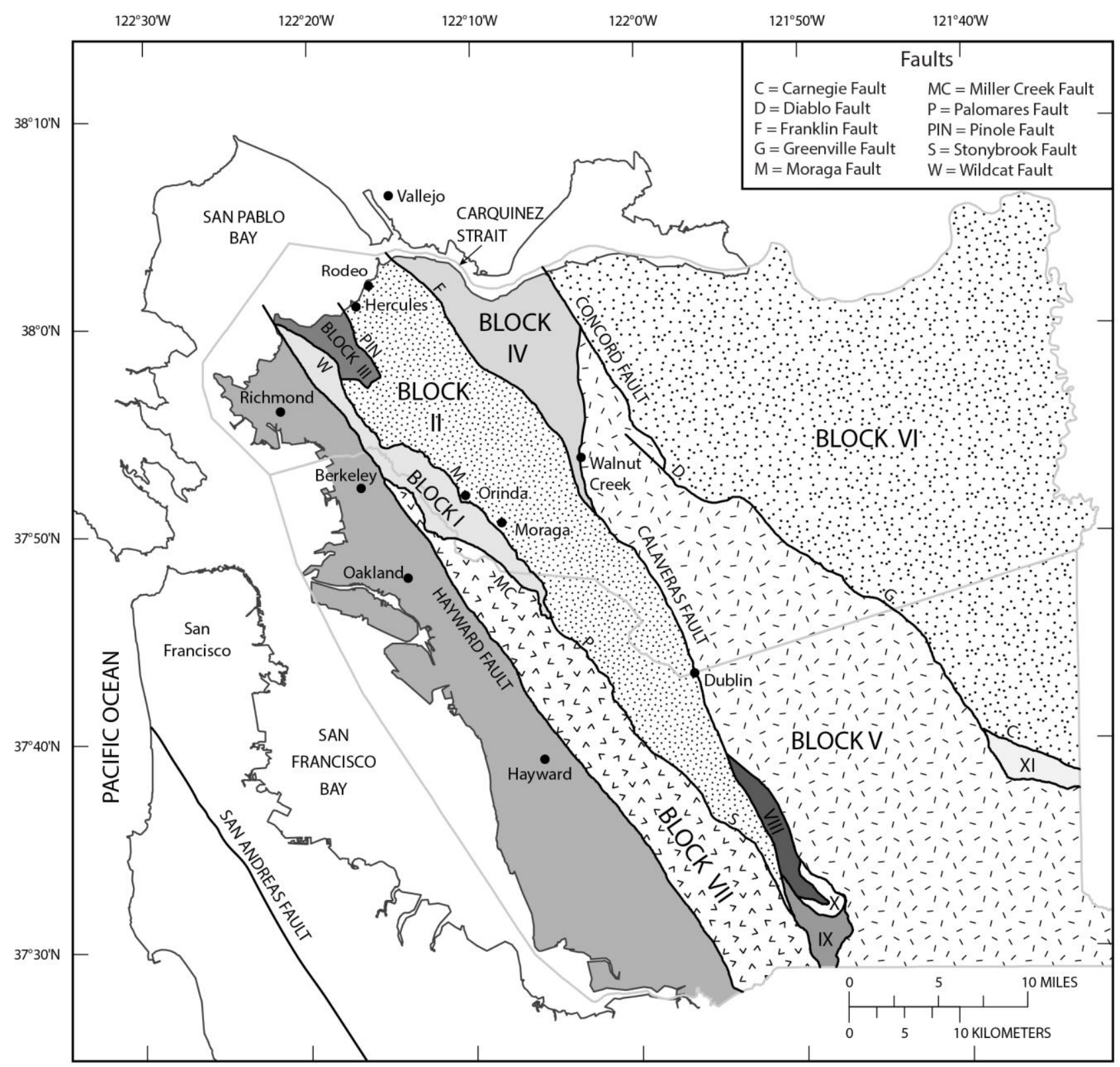

Figure 1. Map of the San Francisco Bay area showing the tectonic blocks into which the area is subdivided by faults (modified from Graymer and others, 1994a, b, 1996). 


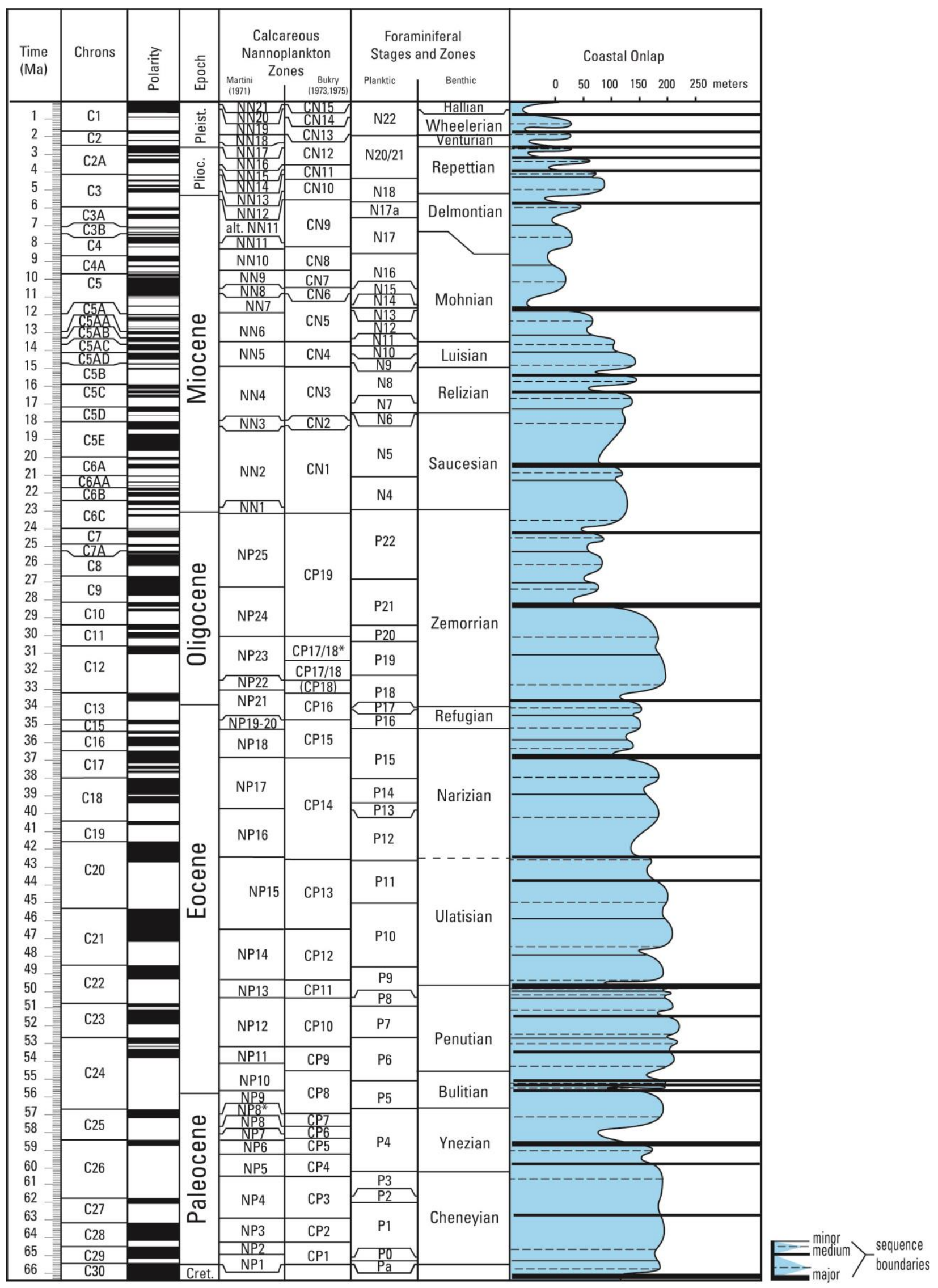


Figure 2. (Preceding page) California Cenozoic biostratigraphic framework from McDougall (2008). The international time scale shown here is from Gradstein and others (2004), Gradstein and Ogg (2005), and the International Commission on Stratigraphy (www.stratigraphy.org) with modifications of the Pliocene/Pleistocene boundary after Gibbard and others (2010). The international time scale is correlated with the paleomagnetic chrons (Berggren, 1972; Berggren and others, 1985, 1985, 2000; Berggren and Pearson, 2005); calcareous nannofossil zones of Bukry $(1973,1975)$, Okada and Bukry (1980), and Martini $(1970,1971)$; planktic foraminiferal zones of Blow (1969, 1979), Berggren and Miller (1989), and Berggren (1972); California benthic foraminiferal zonations as proposed in this study and derived from the work of Schenck and Kleinpell (1936), Laiming (1939), Goudkoff (1945), Loeblich (1958), Natland (1952), Wissler (1943, 1958), Kleinpell (1938), Mallory (1959), McDougall (1980, 1988, 1989, 1991, 1993), Almgren and others (1988), Blake (1991), Bartow (1992), and Sliter and others (1993); and the coastal onlap fluctuations of Haq and others (1987a,b, 1988), modified by Johnson and others (2005). Lines on coastal onlap curve indicate sequence boundaries-heavy solid lines are major sequence boundaries, moderate solid lines are medium sequence boundaries, and thin solid lines are minor sequence boundaries. Dashed lines indicate maximum coastal onlap. Pleist., Pleistocene, Plioc., Pliocene.

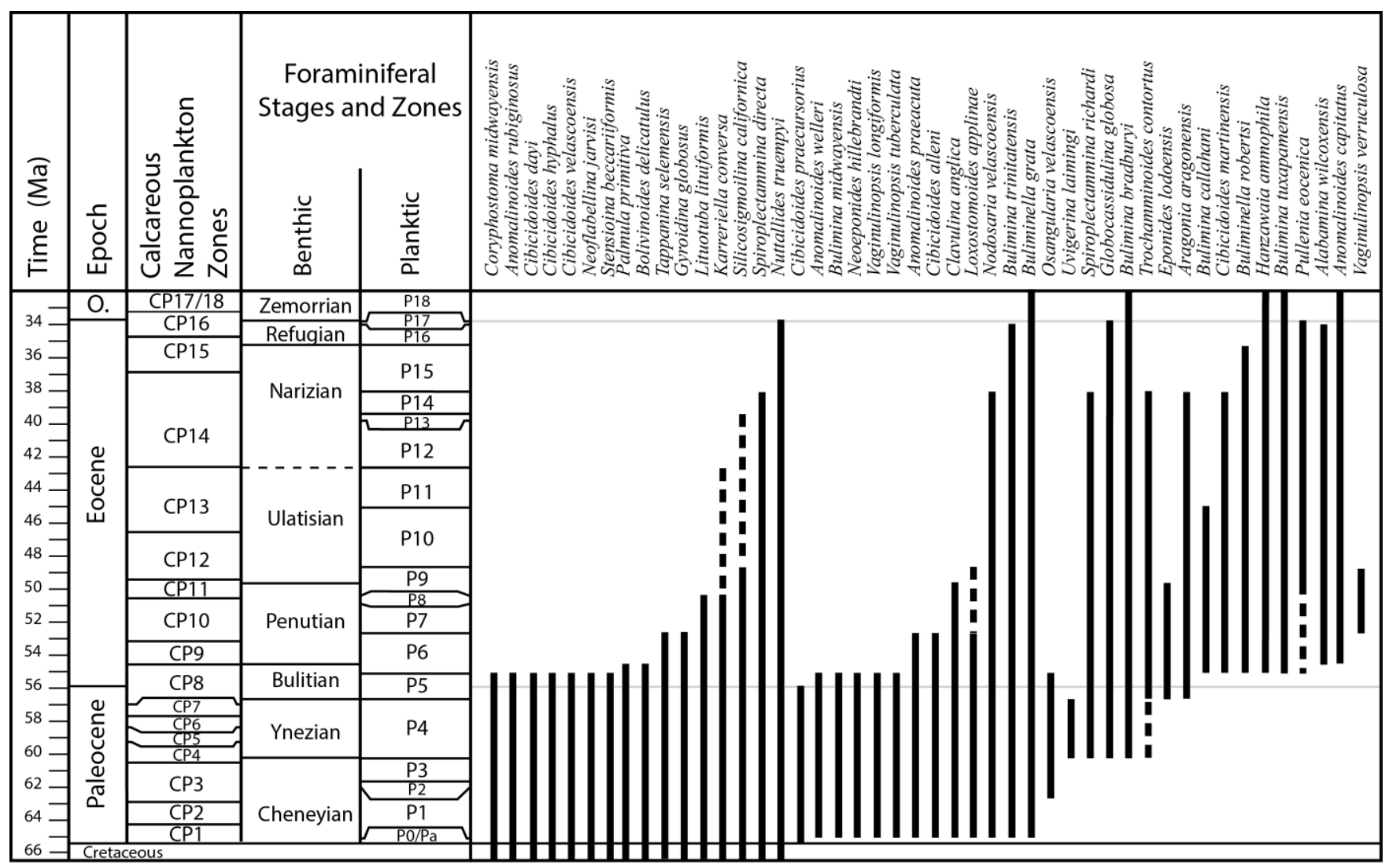

Figure 3. Ranges of Paleocene and some Eocene age-diagnostic benthic foraminifers. The ranges of benthic foraminiferal species are plotted against the time scale shown in figure 2. The ranges of the species are compiled from various literature sources given in the appendix. Solid line indicates documented range of the species; dashed line indicates questionable range of species. O., Oligocene. Foraminiferal stages and zones are given in figure 2. 


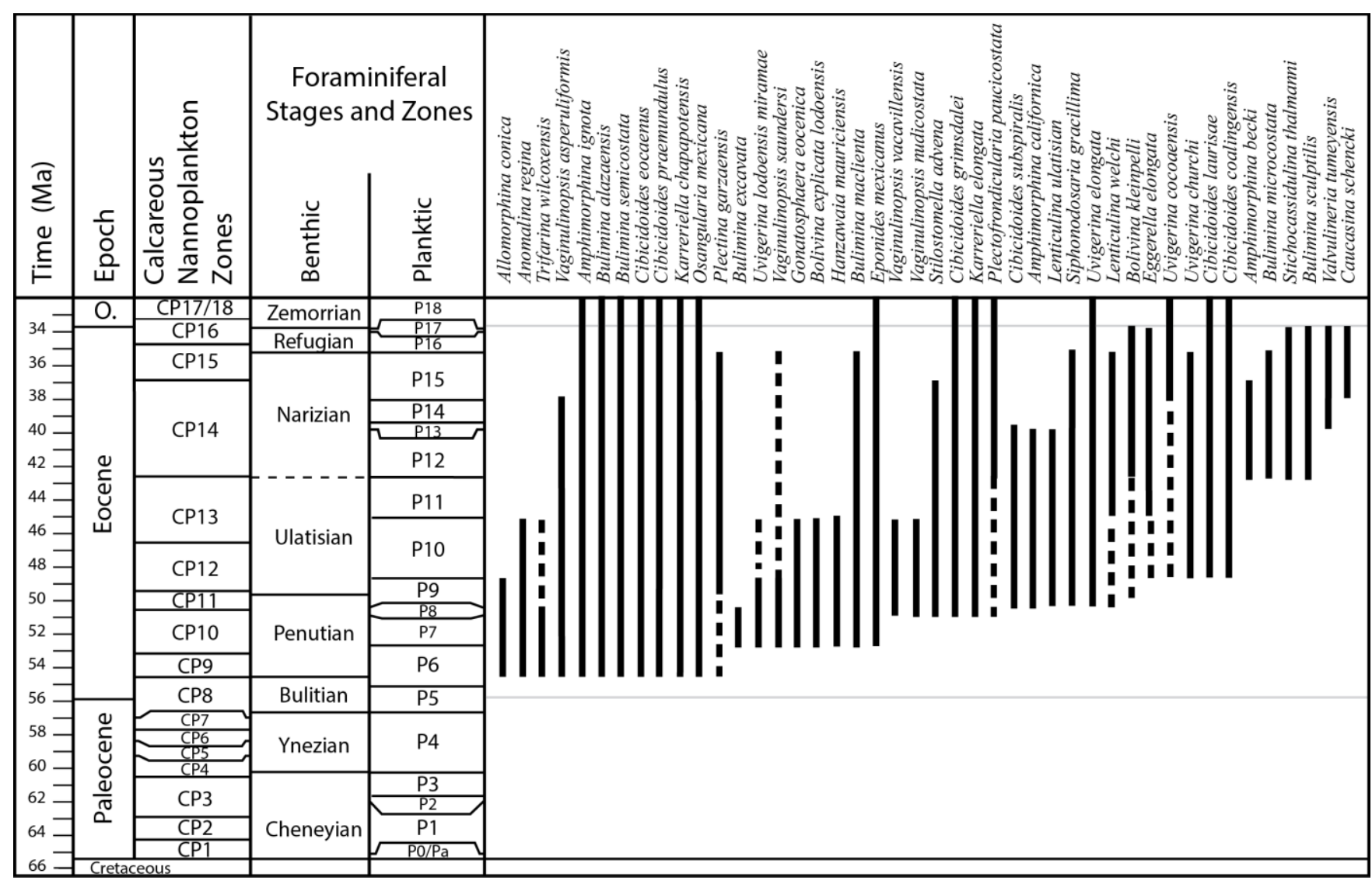

Figure 4. Ranges of Eocene age-diagnostic benthic foraminifers. The ranges of benthic foraminiferal species are plotted against the time scale shown in figure 2 . The ranges of the species are compiled from various literature sources given in the appendix. Solid line indicates documented range of the species; dashed line indicates questionable range of species. O., Oligocene. Foraminiferal stages and zones are given in figure 2.

\section{Ecologic Framework}

Cenozoic environmental interpretations are based on overviews of California benthic foraminifers by Ingle (1980), Ingle and Keller (1980), and Blake (1981, 1991); a study of Atlantic Paleogene benthic foraminifers by Tjalsma and Lohmann (1983); and studies of cosmopolitan benthic foraminifers by Douglas (1981), Woodruff (1985), and van Morkhoven and others (1986). Along the continental margin, these environmental interpretations indicate depth, water mass, or various physical properties such as temperature, salinity, or oxygen content. Depths associated with the bathymetric biofacies follows Ingle (1980): inner neritic (0-50 m), outer neritic (50-150 m), upper bathyal (150-500 m), upper middle bathyal (500-1,500 m), lower middle bathyal (1,500-2,000 m), lower bathyal (2,000-4,000 m), and abyssal (>4,000 m). Ecologic preferences of diagnostic benthic foraminifers are given in the appendix. Correlation of the California biostratigraphic framework to the international time scale (fig. 2) also allows correlation to the global sea level curve (Haq and others, 1987a, b, 1988; Johnson and others 2005) and thus inferences can be made about the global sea level. 


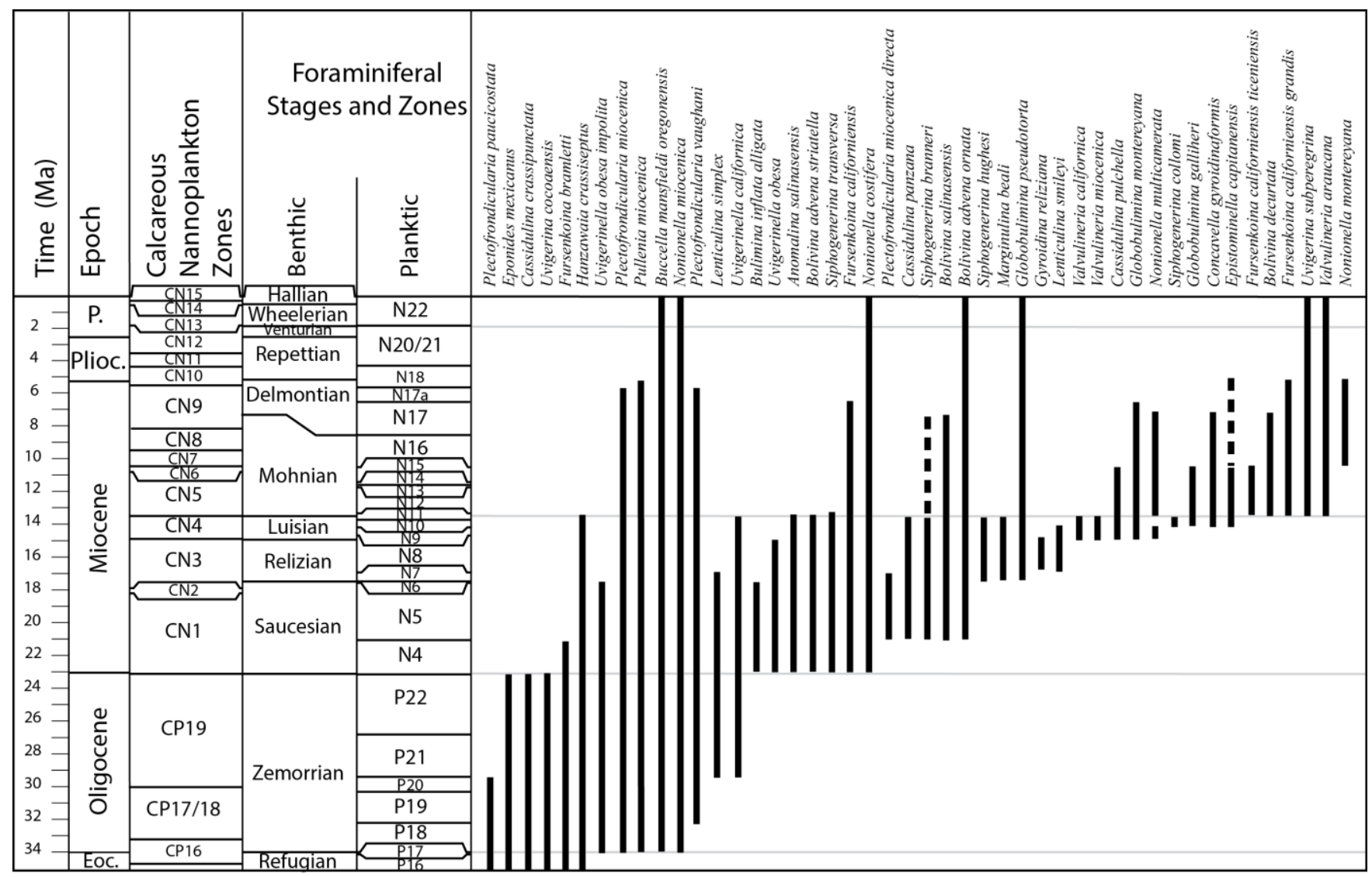

Figure 5. Ranges of Oligocene and Neogene age-diagnostic benthic foraminifers. The ranges of benthic foraminiferal species are plotted against the time scale shown in figure 2 . The ranges of the species are compiled from various literature sources given in the appendix. Solid line indicates documented range of the species; dashed line indicates questionable range of species. P., Pleistocene; Plioc., Pliocene; Eoc., Eocene. Foraminiferal stages and zones are given in figure 2.

\section{Materials and Methods}

Benthic foraminifers samples collected for this study were processed with kerosene and Quaternary-O, washed through a $250-\mu \mathrm{m}$ screen, and dried at low temperatures $\left(<40{ }^{\circ} \mathrm{C}\right)$. The entire sample or a known split was picked and benthic foraminifers identified from the $>150-\mu \mathrm{m}$ fraction. Other samples examined in this study were collected primarily by U.S. Geological Survey geologists and processed in the micropaleontology laboratory. Although the processing and picking techniques have varied over the years, samples processed and examined since approximately 1980 used the techniques discussed above. All picked slides were reexamined in order to provide consistent taxonomy. A comprehensive list of species identified in this study is given in the Taxonomic Notes. Information given for each species includes the first describer, a recent or thorough taxonomic reference, age range, and ecologic attributes. The presence of planktic foraminifers and other organic remains were also noted. Slides are on file with the U.S. Geological Survey Micropaleontology Laboratory in Flagstaff, Arizona. 


\section{Discussion of Tertiary Stratigraphy}

\section{Block I}

Although the Hayward Fault Zone is a major feature in this block, Graymer and others (1994a) show the western boundary of Block I extending across this fault zone into San Pablo and San Francisco Bay. The Wildcat and Moraga Faults mark the eastern boundary (fig. 1). The north boundary is along the Carquinez Strait and the south boundary is the intersection of the Moraga and Miller Creek Faults. This block corresponds to the western portion of the East Bay Hills block of Buising and Walker (1995) and the northern portion of the Caldecott block (Graham and others, 1984). The Tertiary section of Block I is thought to unconformably overlie the Great Valley sequence of Jurassic and Late Cretaceous age based on the presence of fault-bounded slivers of Great Valley sequence intercalated within the Tertiary section. The Paleogene section of Block I is composed of three fault bounded, unnamed units (Ta, Tes/Tsh, and Tsm/Tgs), whereas the Neogene section is composed of six units (unnamed unit Tush, Claremont chert of Graymer, 2000, Orinda, Moraga, Siesta Formations and Bald Peak Basalt; fig. 6) (Graymer and others, 1994a, 1996). The bases of the Claremont chert and Orinda Formation are fault bounded everywhere (Graymer, 2000). No microfossil samples were examined from the portion of the block west of the Hayward Fault Zone.

Unit Ta, an unnamed glauconitic sandstone interbedded with mudstone and fine-grained sandstone, is restricted to a small, fault-bounded area in the Oakland Hills (Graymer and others, 1996; Graymer, 2000). This unit was assigned to the Paleocene based on stratigraphic position and the presence of Paleocene-like mollusks and corals (D.L. Jones, oral commun., 1996). No microfossil samples were examined from this unit.

Unit Tes is an unnamed green and maroon foraminifer-rich mudstone (Graymer and others, 1996; Graymer, 2000); it includes unit Tsh of Graymer and others (1994a), an unnamed greenish gray shale in Contra Costa County. Unit Tes/Tsh is considered Eocene in age but its relationship to the adjacent units is unknown as it is bounded above and below by faults (Graymer and others, 1996). Sixteen microfossil samples were examined from this unit: five samples contained no benthic foraminifers or nondiagnostic arenaceous foraminifers, nine samples contained foraminifers, and two samples were problematic (table 3). The problematic samples were taken from sediments identified as unit Tes by Graymer and others (1996; Graymer, 2000). Sample Mf526 contains Globotruncana arca and is therefore Cretaceous in age. Sample Mf7869 was identified as the Sobrante Sandstone by D.H. Radbruch (unpublished notes) and contains specimens identified as Uvigerina and Uvigerinella, which indicate a Miocene age.

Fossiliferous samples from unit Tes/Tsh contain microfossil assemblages that are late early Eocene in age and assigned to the Penutian through early Ulatisian Stages coeval with planktic foraminiferal zones P7 through P10 (table 3). The early Eocene age is based on the first, last, or restricted appearances of Bulimina callahani, B. macilenta, Clavulina anglica, Glomospira charoides, Karreriella conversa, Loxostomoides applinae, Plectofrondicularia paucicostata, Trifarina wilcoxensis, and Uvigerina lodoensis miriamae. In addition to the early Eocene species, samples Mf7843, Mf7844, and Mf7846, which are from the same general location, contain species that first appear in the latest early Eocene Ulatisian Stage. These age-diagnostic species include Bulimina microcostata, Cibicidoides laurisae, Eggerella cf. E. elongata, Spiroplectammina tejonensis, and Vaginulinopsis mudicostata (table 3). Deposition of unit Tes/Tsh occurred at lower bathyal to abyssal depths $(>2,000 \mathrm{~m})$, although transported shelf specimens and arenaceous species are more common in the younger samples of this unit. 


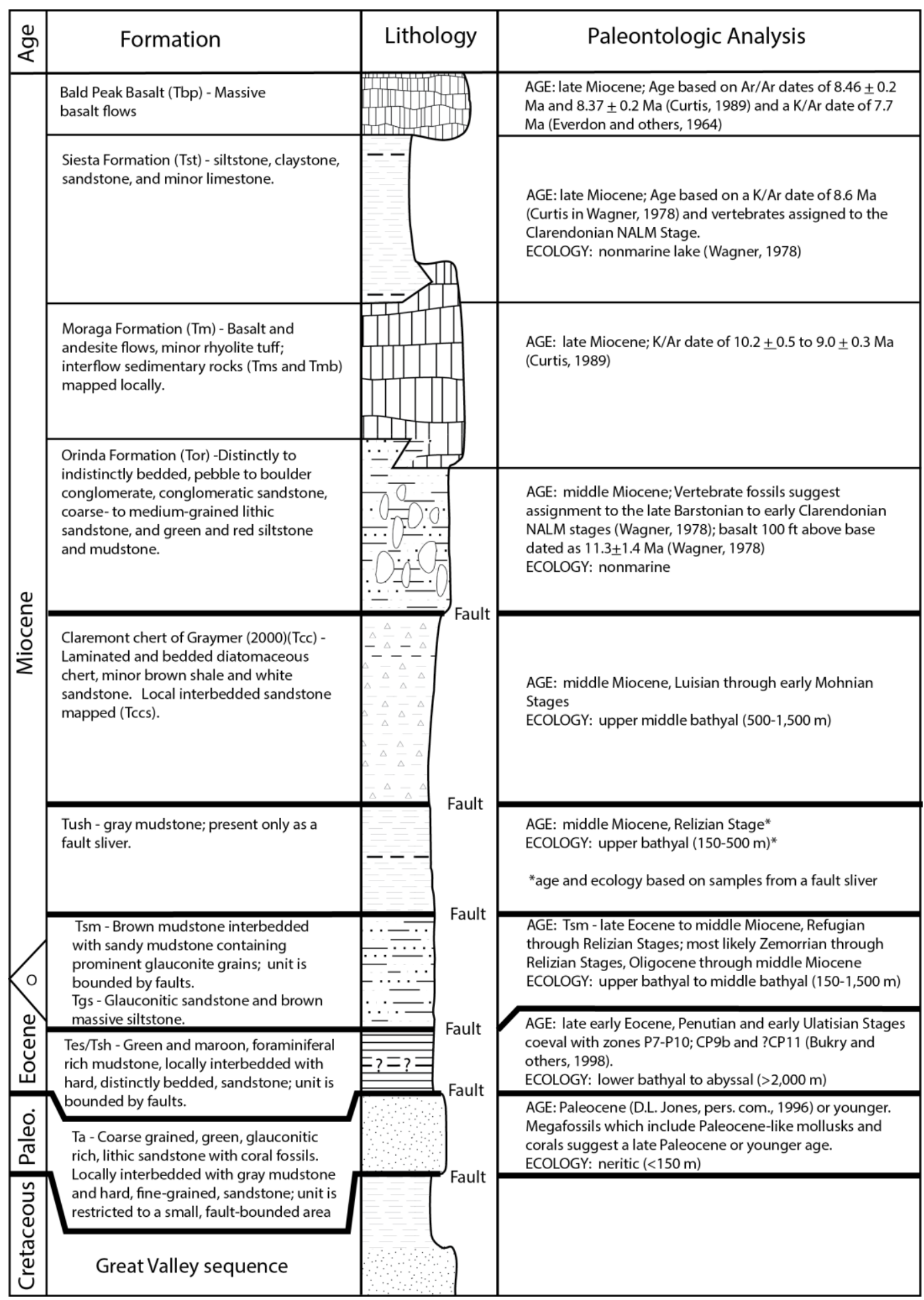

Figure 6. Composite columnar section for Block I. Formation names are from Graymer and others (1994a,b, 1996; Graymer, 2000). Age and environmental interpretations are summarized from the text. O, Oligocene. 
Calcareous nannoplankton from unit Tes samples also group into two distinct early Eocene ages. Samples Mf8575, Mf8576, and Mf8601 are assigned to the early Eocene zone CP9b and samples Mf7843, Mf7844, and Mf7846 are questionably assigned to the early Eocene zone CP11 (Bukry and others, 1998). These interpretations support the early Eocene, Penutian through early Ulatisian Stage assignment of unit Tes by foraminifers.

The next unit in the Block I sequence is comprised of unnamed brown mudstone and sandstone (Tsm of Graymer and others, 1996; Tgs of Graymer and others, 1994a). Three microfossil samples were examined from unit Tgs and 47 samples from unit Tsm of Graymer and others (1996) and Graymer (2000). All samples from unit Tgs (Mf8091, Mf8092, and Mf8093) and 32 samples from the unit Tsm contained no foraminifers or contained only long-ranging nondiagnostic foraminifers. Fifteen samples, which contained foraminiferal assemblages, range in age from Eocene to middle Miocene (table 4).

Three samples collected from unit Tsm suggest an Eocene age: Mf8834, Mf8120, and Mf8412. The foraminiferal fauna in Mf8834 is composed primarily of arenaceous species that commonly occur in the early Eocene but are not restricted to this interval, and Bulimina macilenta, which ranges throughout the Eocene. Sample Mf8120, which contains no foraminifers, is assigned to the middle Eocene zone CP13 based on calcareous nannoplankton (Bukry and others, 1998). This sample was taken adjacent to a fault that separates units Tes and Tsm and may actually be from unit Tes. Sample Mf8412 is late Eocene in age and assigned to the Refugian Stage based on the presence of Uvigerina cocoaensis and Stichocassidulina thalmani, whose ranges overlap in the Refugian Stage. It was taken near the contact between units Tsm and Tush of Graymer and others $(1996,2000)$ and is described as a gray mudstone rather than a glauconitic mudstone; it may be from unit Tush of Graymer and others (1996) and Graymer (2000).

Table 3. Benthic foraminifers from unit Tes/Tsh unit of Graymer and others (1994a, 1996, 2000) of Block I.

[Taxonomy of species is given in the section entitled "Taxonomic Notes." $\mathrm{X}$ indicates the species is present; cf. indicates that the specimens resemble or compare to the species. Samples that are barren of foraminifers or contain only long-ranging nondiagnostic arenaceous foraminifers include: Mf7845, Mf8551, Mf8602, Mf8603, and Mf8604. Problematic samples include Mf526, which is Cretaceous in age, and Mf7869, which is Miocene in age]

\begin{tabular}{|c|c|c|c|c|c|c|c|c|c|}
\hline $\begin{array}{c}\text { Benthic foraminifers from unit } \\
\text { Tes/Tsh unit of Graymer and others } \\
\text { (1994a, 1996; Graymer, 2000) in } \\
\text { Block I }\end{array}$ & 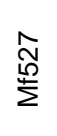 & $\begin{array}{l}\mathscr{N} \\
\stackrel{\rho}{\rho} \\
\sum\end{array}$ & $\begin{array}{l}8 \\
0 \\
\sum^{\circ}\end{array}$ & 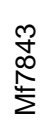 & $\begin{array}{l}\stackrel{+}{+} \\
\stackrel{\infty}{*} \\
\stackrel{2}{\Sigma}\end{array}$ & $\begin{array}{l}0 \\
+ \\
\infty \\
\stackrel{N}{L} \\
\sum\end{array}$ & $\begin{array}{l}\stackrel{10}{N} \\
\stackrel{\infty}{2} \\
\stackrel{\infty}{\Sigma}\end{array}$ & $\begin{array}{l}0 \\
2 \\
\infty \\
\infty \\
\sum\end{array}$ & $\begin{array}{l}\bar{\delta} \\
\mathbb{0} \\
\dot{\infty} \\
\Sigma\end{array}$ \\
\hline Alabamina wilcoxensis & - & - & $x$ & - & - & - & - & - & - \\
\hline Allomorphina conica & - & - & - & - & - & $x$ & - & $x$ & $x$ \\
\hline Ammodiscus incertus & - & - & - & $x$ & - & $x$ & - & $x$ & $x$ \\
\hline Ammodiscus spp. & $x$ & - & - & - & - & $x$ & - & - & - \\
\hline Amphimorphina californica & - & - & $x$ & - & - & - & - & - & - \\
\hline Amphimorphina ignota & - & $x$ & $x$ & $x$ & - & $x$ & - & - & $x$ \\
\hline Anomalinoides capitatus & - & - & - & - & - & $x$ & $x$ & $x$ & $x$ \\
\hline Anomalina garzaensis & - & - & - & $x$ & - & - & - & - & - \\
\hline Anomalina keenae & - & - & - & - & $x$ & $x$ & - & $x$ & $x$ \\
\hline Anomalina regina & - & - & - & $x$ & $x$ & $x$ & - & $x$ & - \\
\hline Aragonia aragonensis & - & $x$ & - & - & - & - & - & - & $x$ \\
\hline Bathysiphon eocenicus & - & $x$ & - & - & - & - & - & - & - \\
\hline Bathysiphon santecruis & - & - & - & - & - & $x$ & - & - & - \\
\hline Bathysiphon spp. & - & - & - & $x$ & $x$ & $\mathrm{x}$ & $x$ & $x$ & $x$ \\
\hline Boldia hodgei & - & - & - & - & $x$ & - & - & - & - \\
\hline Bolivina explicata lodoensis & - & $x$ & - & - & - & - & - & - & - \\
\hline Bolivina incrassata of Mallory & - & - & - & - & - & - & - & $x$ & - \\
\hline Bulimina alazaensis & - & - & - & $x$ & - & $x$ & - & - & $x$ \\
\hline
\end{tabular}




\begin{tabular}{|c|c|c|c|c|c|c|c|c|c|}
\hline $\begin{array}{l}\text { Benthic foraminifers from unit } \\
\text { Tes/Tsh unit of Graymer and others } \\
\text { (1994a, 1996; Graymer, 2000) in } \\
\text { Block I }\end{array}$ & 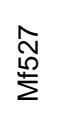 & 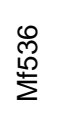 & $\begin{array}{l}8 \\
\stackrel{\infty}{\Sigma} \\
\sum^{L}\end{array}$ & 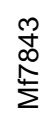 & $\begin{array}{l}\text { 寸 } \\
\text { D } \\
\stackrel{1}{\Sigma}\end{array}$ & $\begin{array}{l}0 \\
+ \\
\infty \\
1 \\
\sum \\
\Sigma\end{array}$ & 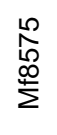 & $\begin{array}{l}0 \\
1 \\
10 \\
\infty \\
\Sigma\end{array}$ & $\begin{array}{l}\bar{\delta} \\
\varnothing \\
\infty \\
\Sigma\end{array}$ \\
\hline Bulimina bradburyi & - & - & - & $x$ & $x$ & - & - & $x$ & - \\
\hline Bulimina callahani & - & - & - & - & - & $x$ & - & $x$ & - \\
\hline Bulimina macilenta & - & - & - & $x$ & $x$ & $x$ & $x$ & $x$ & $x$ \\
\hline Bulimina microcostata & - & - & - & - & $x$ & - & - & - & - \\
\hline Bulimina spp. & - & $x$ & - & - & - & - & - & - & - \\
\hline Bulimina trinitatensis & - & - & - & $x$ & - & $x$ & - & $x$ & - \\
\hline Buliminella grata & - & - & - & - & - & - & $x$ & $x$ & $x$ \\
\hline Chrysalongonium elongatum & - & - & - & $x$ & - & $x$ & - & $x$ & $x$ \\
\hline Chrysalongonium longiscatatum & - & - & - & - & - & $x$ & - & - & - \\
\hline Chrysalongonium spp. & - & - & - & $x$ & - & - & - & - & - \\
\hline Chrysalongonium tenuicostatum & - & - & - & $x$ & - & $x$ & $x$ & $x$ & $x$ \\
\hline Cibicides beatus & - & - & - & $X$ & - & - & - & - & - \\
\hline Cibicides felix & - & - & - & $x$ & $x$ & - & - & - & - \\
\hline Cibicides martinezensis & - & - & - & - & - & $x$ & - & - & - \\
\hline Cibicidoides coalingensis & - & - & - & $x$ & - & - & - & - & - \\
\hline Cibicidoides eocaenus & - & $x$ & - & - & - & - & $x$ & $x$ & $x$ \\
\hline Cibicidoides eponidiformis & - & - & - & - & - & - & - & $x$ & $x$ \\
\hline Cibicidoides laurisae & - & - & - & - & - & $x$ & - & - & - \\
\hline Cibicidoides praemundulus & - & - & - & $x$ & $x$ & - & - & - & $x$ \\
\hline Cibicidoides subspiratus & - & - & - & $x$ & $x$ & $x$ & - & - & $x$ \\
\hline Cibicidoides spp. & - & - & $x$ & $x$ & - & - & - & $x$ & $x$ \\
\hline Cibicidoides venzuelanus & - & - & - & $x$ & - & - & - & - & - \\
\hline Clavulina anglica & - & $x$ & - & - & $x$ & $x$ & $x$ & $x$ & $x$ \\
\hline Clavulinoides califonicus (C. sp. A) & - & $x$ & - & - & - & - & - & - & - \\
\hline Cribrostomoides trintatensis & - & - & - & - & - & - & $x$ & - & $x$ \\
\hline Cyclammina samanica & - & - & - & - & - & - & $x$ & - & $x$ \\
\hline Cyclammina simiensis & - & - & - & - & - & $x$ & - & - & - \\
\hline Dentalina colei & - & - & - & $x$ & $x$ & $x$ & - & - & - \\
\hline Dentalina communis & - & - & - & $x$ & $x$ & - & $x$ & $x$ & $x$ \\
\hline Dentalina consobrina & - & - & - & $x$ & - & - & - & - & - \\
\hline Dentalina delicatula & - & - & - & $x$ & - & - & - & - & - \\
\hline Dentalina jacksonensis & - & - & - & - & $x$ & - & - & - & - \\
\hline Dentalina spp. & - & - & $x$ & - & - & $x$ & - & - & - \\
\hline Dorothia bulletta & - & - & - & - & $x$ & $x$ & - & - & - \\
\hline Dorothia principiensis & - & $x$ & - & - & - & $x$ & $x$ & $x$ & $x$ \\
\hline Eggerella elongata & - & - & - & - & - & cf. & - & - & - \\
\hline Eponides lodoensis & - & - & - & $x$ & $x$ & $x$ & - & - & - \\
\hline Eponides lotus & - & - & - & - & - & - & - & - & $x$ \\
\hline Fissurina marginata & - & - & - & - & - & $\mathrm{x}$ & - & - & - \\
\hline Fursenkoina bramletti & - & - & - & - & - & $x$ & - & $x$ & - \\
\hline Gaudryina coalingensis & - & $x$ & - & $x$ & - & - & - & - & - \\
\hline Gaudryina pyramidata & - & $x$ & - & - & - & - & - & - & - \\
\hline Globulina lacrima & - & $x$ & - & - & - & - & - & - & - \\
\hline Glomospira charoides & $x$ & - & - & - & - & - & $x$ & $x$ & $x$ \\
\hline Gonatosphaera eocenica & - & - & - & $x$ & $x$ & - & - & - & - \\
\hline Guttulina irregularis & - & - & - & $x$ & - & - & - & - & - \\
\hline Guttulina spp. & - & - & - & - & - & $x$ & - & - & - \\
\hline Gyroidina soldanii & - & $x$ & - & - & - & - & $x$ & $x$ & $x$ \\
\hline Hanzawaia ammophila & - & - & - & $x$ & - & - & - & - & - \\
\hline Hanzawaia mauricensis & - & $x$ & - & - & $x$ & - & - & - & - \\
\hline Haplophragmoides eggeri & - & - & - & - & - & $\mathrm{x}$ & $x$ & - & - \\
\hline Haplophragmoides glabra & - & - & - & - & - & - & - & - & $x$ \\
\hline
\end{tabular}




\begin{tabular}{|c|c|c|c|c|c|c|c|c|c|}
\hline $\begin{array}{l}\text { Benthic foraminifers from unit } \\
\text { Tes/Tsh unit of Graymer and others } \\
(1994 a, 1996 ; \text { Graymer, 2000) in } \\
\text { Block I }\end{array}$ & 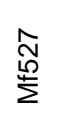 & $\begin{array}{l}\mathscr{N} \\
\stackrel{\rho}{\rho} \\
\stackrel{\Sigma}{\Sigma}\end{array}$ & $\begin{array}{l}8 \\
\stackrel{\infty}{\Sigma} \\
\sum^{L}\end{array}$ & 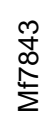 & 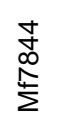 & $\begin{array}{l}0 \\
+ \\
\infty \\
1 \\
\sum \\
\Sigma\end{array}$ & $\begin{array}{l}n \\
\stackrel{1}{n} \\
\infty \\
\infty \\
\sum\end{array}$ & $\begin{array}{l}0 \\
1 \\
10 \\
\infty \\
\Sigma\end{array}$ & $\begin{array}{l}\bar{\delta} \\
\varnothing \\
\infty \\
\Sigma\end{array}$ \\
\hline Haplophragmoides spp. & - & - & $x$ & $x$ & - & $x$ & - & - & - \\
\hline Hyperammina elongata & - & - & - & - & $x$ & - & - & - & - \\
\hline Karreriella conversa & - & - & - & - & - & - & $x$ & $x$ & $x$ \\
\hline Karreriella elongata & - & - & - & $x$ & - & - & - & - & - \\
\hline Lagena spp. & - & - & - & - & - & - & $x$ & $x$ & $x$ \\
\hline Lagena vulgaris & - & - & - & $x$ & - & $x$ & - & - & - \\
\hline Lenticulina arcuatostriata & - & - & - & - & - & - & - & - & $x$ \\
\hline Lenticulina caritae & - & - & - & - & $x$ & - & - & - & $x$ \\
\hline Lenticulina limbosa & - & - & - & - & - & - & - & - & $x$ \\
\hline Lenticulina limbosa hockleyensis & - & - & - & - & $x$ & - & - & - & - \\
\hline Lenticulina pseudocultrata & - & - & - & $x$ & $x$ & - & - & - & $x$ \\
\hline Lenticulina pseudovortex & - & - & - & - & - & - & - & - & $x$ \\
\hline Lenticulina spp. & - & - & $x$ & $x$ & $x$ & $x$ & - & - & $x$ \\
\hline Lenticulina terryi & - & - & - & $x$ & - & - & - & - & $x$ \\
\hline Lenticulina vortex & - & - & - & - & - & $x$ & - & - & - \\
\hline Lituotuba lituiformis & - & - & - & - & - & $x$ & - & - & - \\
\hline Loxostomoides applinae & - & - & - & $x$ & - & - & - & $x$ & - \\
\hline Marginulina exima & - & - & - & $x$ & - & $x$ & - & - & - \\
\hline Marginulina subbullata & - & - & - & $x$ & - & - & - & - & - \\
\hline Marginulina spp. & - & - & - & $x$ & - & - & - & - & $x$ \\
\hline Nonionella spp. & - & - & - & - & - & $x$ & - & $x$ & $x$ \\
\hline Nodosaria latejugata & - & - & - & $x$ & - & - & - & $x$ & - \\
\hline Nodosaria longiscata & - & - & - & $x$ & $x$ & - & $x$ & $x$ & - \\
\hline Nodosaria spp. & - & - & $x$ & - & - & - & $x$ & - & - \\
\hline Nodosarella advena & - & - & - & $x$ & $x$ & - & - & $x$ & - \\
\hline Nodosarella atlantisae hispidula & - & - & - & - & - & $x$ & - & - & - \\
\hline Nuttaloides truempyi & - & - & - & $x$ & - & $x$ & $x$ & $x$ & $x$ \\
\hline Oridorsalis umbonatus & - & - & - & $x$ & - & $x$ & $x$ & $x$ & $x$ \\
\hline Orthomorphina rohri & - & - & - & $x$ & - & - & - & - & - \\
\hline Osangularia mexicana & - & $x$ & - & $x$ & $x$ & $x$ & $x$ & $x$ & $x$ \\
\hline Planularia spp. & - & - & - & - & - & - & - & $x$ & - \\
\hline Planulina truncana & - & - & - & - & - & - & - & - & $x$ \\
\hline Plectina garzaensis & - & - & - & - & - & $x$ & - & - & $x$ \\
\hline Plectofrondicularia paucicostata & - & - & - & $x$ & $x$ & - & - & - & - \\
\hline Pleurostomella acuta & - & $x$ & - & $x$ & - & $x$ & - & $x$ & $x$ \\
\hline Pleurostomella gredalensis & - & - & - & $x$ & - & - & - & $x$ & $x$ \\
\hline Pleurostomella spp. & - & $x$ & - & - & $x$ & $x$ & - & $x$ & - \\
\hline Pseudonodosaria conica & - & - & - & $x$ & $x$ & $x$ & $x$ & $x$ & $x$ \\
\hline Pseudonodosaria inflata & - & - & - & $x$ & $x$ & $x$ & - & - & - \\
\hline Pseudonodosaria spp. & - & - & - & $x$ & - & - & - & - & - \\
\hline Pullenia eocenica & - & $x$ & - & - & - & $x$ & - & $x$ & $x$ \\
\hline Pullenia eocenica & - & - & - & cf. & - & - & - & - & - \\
\hline Pullenia quinqueloba & - & - & - & $x$ & - & $x$ & - & - & $x$ \\
\hline Pullenia salisburyi & - & - & - & - & - & - & $x$ & $x$ & $x$ \\
\hline Pyrulina cylindroides & - & - & - & - & - & - & - & $x$ & $x$ \\
\hline Pyrulina spp. & - & - & - & - & - & - & - & - & $x$ \\
\hline Reophax pilulifera & - & - & - & - & - & $x$ & - & - & - \\
\hline Saccammina spp. & - & - & - & - & - & $x$ & - & $x$ & - \\
\hline Schenckiella spp. & - & - & - & - & $x$ & - & - & - & - \\
\hline Silicosigmoilina californica & $x$ & - & - & - & $x$ & $x$ & $x$ & $x$ & $x$ \\
\hline Siphonodosaria gracillima & - & - & - & - & - & - & - & - & $x$ \\
\hline Siphonodosaria spp. & - & - & - & - & - & - & - & - & $x$ \\
\hline
\end{tabular}




\begin{tabular}{|c|c|c|c|c|c|c|c|c|c|}
\hline $\begin{array}{c}\text { Benthic foraminifers from unit } \\
\text { Tes/Tsh unit of Graymer and others } \\
\text { (1994a, 1996; Graymer, 2000) in } \\
\text { Block I }\end{array}$ & $\begin{array}{l}\hat{N} \\
\stackrel{N}{N} \\
\sum\end{array}$ & 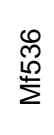 & $\begin{array}{l}\stackrel{\bigcirc}{\$} \\
\stackrel{\infty}{\Sigma}\end{array}$ & 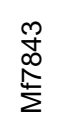 & $\begin{array}{l}\text { J } \\
\stackrel{D}{\infty} \\
\stackrel{p}{\Sigma}\end{array}$ & $\begin{array}{l}\varphi \\
+ \\
\infty \\
\stackrel{p}{\Sigma} \\
\Sigma\end{array}$ & $\begin{array}{l}\stackrel{1}{N} \\
\stackrel{0}{\infty} \\
\stackrel{\infty}{\Sigma}\end{array}$ & $\begin{array}{l}\mathscr{Q} \\
\stackrel{2}{ } \\
\infty \\
\Sigma\end{array}$ & $\begin{array}{l}\bar{\delta} \\
\mathbb{\infty} \\
\stackrel{\infty}{\Sigma}\end{array}$ \\
\hline Spiroplectammina directa & $x$ & - & - & - & - & $x$ & - & $x$ & $x$ \\
\hline Spiroplectammina richardi & - & $x$ & $x$ & $x$ & - & - & $x$ & $x$ & $x$ \\
\hline Spiroplectammina tejonensis & - & - & - & - & - & $x$ & - & - & - \\
\hline Stilostomella adolphina & - & - & - & - & - & $x$ & - & - & - \\
\hline Stilostomella lepidula & - & - & - & - & $x$ & - & - & $x$ & $x$ \\
\hline Textularia adalta & - & - & - & - & - & - & - & - & $x$ \\
\hline Textularia adalta & - & - & - & - & - & cf. & - & - & - \\
\hline Textularia conica & - & - & - & - & - & - & $x$ & - & $x$ \\
\hline Textularia plummerae & - & - & - & - & - & - & $x$ & $x$ & - \\
\hline Trifarina advena californica & - & $x$ & - & $x$ & - & - & - & - & - \\
\hline Trifarina wilcoxensis & - & - & - & - & $x$ & - & - & - & - \\
\hline Tritaxilina colei & - & - & - & $x$ & - & $x$ & - & - & - \\
\hline Trochammina spp. & - & - & $x$ & - & - & - & - & - & - \\
\hline Trochamminoides contortus & - & - & - & - & - & - & $x$ & $x$ & $x$ \\
\hline Uvigerina lodoensis miriamae & - & - & - & - & - & - & - & - & $x$ \\
\hline Vaginulinopsis asperuliformis & - & $x$ & - & $x$ & $x$ & - & - & - & - \\
\hline Vaginulinopsis nudicostata & - & - & - & - & $x$ & - & - & - & - \\
\hline Verneuilina triangulata & - & $x$ & - & $x$ & $x$ & - & - & - & - \\
\hline Vulvulina curta & - & $x$ & - & $x$ & - & - & - & - & - \\
\hline
\end{tabular}

Table 4. Benthic foraminifers from unit Tsm of Graymer and others (1996; Graymer, 2000) of Block I.

[Taxonomy of species is given in the section entitled "Taxonomic Notes." $\mathrm{X}$ indicates the species is present; cf. indicates that the specimens resemble or compare to the species. Samples from the Tsm unit that are barren of foraminifers or contain only long-ranging, nondiagnostic arenaceous foraminifers include: Mf539, Mf545, Mf637, Mf638, Mf801, Mf808, Mf814, Mf848, Mf849, Mf7785, Mf7786, Mf7787, Mf7839, Mf8094, Mf8096, Mf8097, Mf8098, Mf8099, Mf8100, Mf8101, Mf8118, Mf8119, Mf8120, Mf8123, Mf8124, Mf8382, Mf8383, Mf8384, Mf8385, Mf8386, Mf8413, and Mf8457. Samples Mf8091, Mf8092, and Mf8093 from the Tgs unit are barren of microfossils]

\begin{tabular}{|c|c|c|c|c|c|c|c|c|c|c|c|c|c|c|c|}
\hline $\begin{array}{l}\text { Benthic foraminifers from unit Tsm of } \\
\text { Graymer and others (1996; Graymer, } \\
\text { 2000) of Block I }\end{array}$ & $\begin{array}{l}\text { W } \\
\infty \\
\infty \\
\infty\end{array}$ & 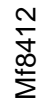 & 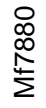 & $\begin{array}{l}\infty \\
\infty \\
\infty \\
\stackrel{\infty}{\Sigma}\end{array}$ & 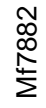 & $\begin{array}{l}\infty \\
\infty \\
\infty \\
\infty \\
\stackrel{\infty}{2} \\
\sum\end{array}$ & 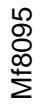 & $\begin{array}{l}\text { N } \\
\stackrel{\infty}{\infty} \\
\stackrel{\infty}{\Sigma}\end{array}$ & $\begin{array}{l}\mathscr{m} \\
\stackrel{\infty}{\infty} \\
\sum_{\Sigma}^{\infty}\end{array}$ & $\begin{array}{l}\stackrel{2}{\infty} \\
\stackrel{\infty}{\Sigma}\end{array}$ & $\begin{array}{l}\hat{\circ} \\
\stackrel{\infty}{\infty} \\
\sum^{\infty}\end{array}$ & $\begin{array}{c}\hat{M} \\
\sum_{\infty}^{\infty}\end{array}$ & $\begin{array}{l}\infty \\
\stackrel{\infty}{\infty} \\
\stackrel{\infty}{\infty}\end{array}$ & $\begin{array}{l}\text { N } \\
\infty \\
\stackrel{\infty}{\Sigma}\end{array}$ & $\begin{array}{l}\mathscr{m} \\
\infty \\
\infty \\
\infty \\
\sum\end{array}$ \\
\hline Bathysiphon eocenicus & $\mathrm{X}$ & $\mathrm{X}$ & - & - & - & - & - & - & - & - & - & - & - & - & - \\
\hline Bathysiphon spp. & - & $x$ & $x$ & - & - & - & $x$ & - & - & - & - & - & - & - & - \\
\hline Bolivina advena striatella & - & - & - & - & - & - & - & - & $\mathrm{X}$ & - & - & - & - & - & - \\
\hline Bolivina cuneiformis & - & - & - & - & - & - & - & - & - & - & - & - & - & $x$ & - \\
\hline Bolivina marginata & - & - & - & - & - & - & - & - & - & - & - & - & - & $x$ & - \\
\hline Bolivina spp. & - & - & - & - & - & - & - & - & - & - & $x$ & $x$ & $x$ & - & - \\
\hline Bulimina lirata & - & $x$ & - & - & - & - & - & - & - & - & - & - & - & - & - \\
\hline Bulimina macilenta & cf. & - & - & - & - & - & - & - & - & - & - & - & - & - & - \\
\hline Buliminella subfusiformis & - & - & - & - & - & - & - & - & - & - & $x$ & - & $x$ & - & - \\
\hline Cassidulina crassipunctata & - & - & - & - & - & - & $x$ & - & - & - & - & - & - & - & - \\
\hline Cibicides spp. & - & - & - & - & - & - & $x$ & - & - & - & - & - & - & - & - \\
\hline Clavulina anglica & - & $x$ & - & - & - & - & - & - & - & - & - & - & - & - & - \\
\hline Cyclammina pacifica & - & - & $x$ & $X$ & - & $x$ & - & - & - & - & - & - & - & - & - \\
\hline Cyclammina spp. & - & - & - & - & $x$ & - & - & - & - & - & - & - & - & - & - \\
\hline Dentalina consobrina & - & $x$ & - & - & - & - & - & - & - & - & - & - & - & - & - \\
\hline Dentalina roemeri & - & & - & - & - & - & - & - & - & - & - & - & $x$ & - & - \\
\hline Dentalina spp. & - & - & - & - & - & - & - & - & - & - & $x$ & - & & $x$ & \\
\hline Epistominella subperuviana & - & - & - & - & - & - & - & - & - & - & - & - & $x$ & - & - \\
\hline Eponides mexicanus & - & $x$ & - & - & - & - & - & - & - & - & - & - & & - & - \\
\hline Fursenkoina californiensis & - & - & - & - & - & - & - & - & $x$ & - & - & $x$ & $x$ & - & - \\
\hline Globobulimina pacifica & - & $x$ & - & - & - & - & - & - & - & - & - & - & & - & - \\
\hline
\end{tabular}




\begin{tabular}{|c|c|c|c|c|c|c|c|c|c|c|c|c|c|c|c|}
\hline $\begin{array}{l}\text { Benthic foraminifers from unit Tsm of } \\
\text { Graymer and others (1996; Graymer, } \\
\text { 2000) of Block I }\end{array}$ & $\begin{array}{l}\underset{N}{ \pm} \\
\infty \\
\infty \\
\sum_{\Sigma}^{\infty}\end{array}$ & $\frac{N}{\underset{\infty}{\infty}}$ & $\begin{array}{l}\infty \\
\infty \\
\infty \\
\stackrel{\infty}{L} \\
\sum\end{array}$ & $\begin{array}{l}\infty \\
\infty \\
\infty \\
\infty \\
\sum \\
\sum\end{array}$ & $\begin{array}{l}\stackrel{N}{\infty} \\
\infty \\
\infty \\
\stackrel{2}{\Sigma}\end{array}$ & $\begin{array}{l}\infty \\
\infty \\
\infty \\
\stackrel{\infty}{\Sigma} \\
\stackrel{L}{\Sigma}\end{array}$ & $\begin{array}{l}\mathscr{2} \\
\stackrel{\circ}{\infty} \\
\sum_{\Sigma}^{\infty}\end{array}$ & $\begin{array}{l}\widetilde{N} \\
\stackrel{\infty}{\Sigma} \\
\sum\end{array}$ & $\begin{array}{l}\mathscr{m} \\
\infty \\
\infty_{\infty}^{\infty}\end{array}$ & $\begin{array}{l}\stackrel{2}{\infty} \\
\stackrel{\infty}{\Sigma} \\
\sum^{\infty}\end{array}$ & $\begin{array}{l}\hat{o} \\
\underbrace{\infty}\end{array}$ & $\begin{array}{l}\hat{m} \\
\infty_{\Sigma}^{L}\end{array}$ & $\begin{array}{l}\infty \\
\stackrel{\infty}{\infty} \\
\infty_{\Sigma}^{\infty}\end{array}$ & $\begin{array}{l}\infty \\
\infty \\
\infty \\
\Sigma \\
\Sigma\end{array}$ & $\begin{array}{l}\infty \\
\infty \\
\infty \\
\stackrel{\infty}{\Sigma}\end{array}$ \\
\hline Globobulimina pseudoaffinis & - & - & - & - & - & - & - & - & - & - & - & $x$ & $\mathrm{x}$ & - & - \\
\hline Globobulimina spp. & - & - & - & - & - & - & $\mathrm{x}$ & - & - & - & - & - & - & - & - \\
\hline Gyroidina octocameratus & - & $\mathrm{x}$ & - & - & - & - & - & - & - & - & - & - & - & - & - \\
\hline Gyroidina spp. & - & - & - & - & - & - & - & - & - & - & - & - & - & $\mathrm{X}$ & $\mathrm{x}$ \\
\hline Haplophragmoides spp. & - & $\mathrm{x}$ & $\mathrm{x}$ & - & - & - & - & - & - & - & - & - & - & - & - \\
\hline Hormosina spp. & $\mathrm{x}$ & - & - & - & - & - & - & - & - & - & - & - & - & - & - \\
\hline Karreriella horrida & $\mathrm{x}$ & - & - & - & - & - & - & - & - & - & - & - & - & - & - \\
\hline Karreriella spp. & - & $\mathrm{x}$ & - & - & - & - & - & - & - & - & - & - & - & - & - \\
\hline Lenticulina inornata & - & $\mathrm{x}$ & - & - & - & - & - & - & - & - & - & - & - & - & - \\
\hline Lenticulina spp. & - & - & - & - & - & - & $\mathrm{x}$ & - & - & $\mathrm{x}$ & - & - & - & - & - \\
\hline Nonionella costifera & - & - & - & - & - & - & - & - & $x$ & - & - & - & - & - & - \\
\hline Nonionella miocenica & - & - & - & - & - & - & - & - & $\mathrm{x}$ & - & - & - & - & - & - \\
\hline Nonionella spp. & - & $\mathrm{x}$ & - & - & - & - & - & - & - & - & - & - & - & - & - \\
\hline Nodosaria longiscata & - & $\mathrm{x}$ & - & - & - & - & - & - & - & - & - & - & - & - & - \\
\hline Nodosaria spp. & - & $\mathrm{x}$ & - & - & - & - & - & - & - & - & - & - & - & - & - \\
\hline Oridorsalis umbonatus & - & $\mathrm{x}$ & - & - & - & - & - & - & - & - & - & - & - & - & - \\
\hline Plectofrondicularia miocenica & - & - & - & - & - & - & - & - & - & - & - & - & - & $\mathrm{x}$ & - \\
\hline Plectofrondicularia miocenica directa & - & - & - & - & - & - & - & - & - & $\mathrm{x}$ & - & - & - & - & - \\
\hline Pseudonodosaria inflata & - & $\mathrm{x}$ & - & - & - & - & - & - & - & - & - & - & - & - & - \\
\hline Praeglobobulimina pupoides & - & $\mathrm{x}$ & - & - & - & - & - & - & - & - & - & - & - & - & - \\
\hline Quinqueloculina spp. & $\mathrm{x}$ & - & - & - & - & - & - & - & - & - & - & - & - & - & - \\
\hline Siphogenerina branneri & - & - & - & - & - & - & - & - & - & - & - & - & $x$ & $\mathrm{x}$ & - \\
\hline Siphogenerina hughesi & - & - & - & - & - & - & - & $\mathrm{x}$ & - & - & - & - & - & - & - \\
\hline Siphogenerina transversa & - & - & - & - & - & - & - & - & - & - & $\mathrm{x}$ & - & - & - & - \\
\hline Stichocassidulina thalmani & - & $\mathrm{x}$ & - & - & - & - & - & - & - & - & - & - & - & - & - \\
\hline Uvigerina cocoaensis & - & $\mathrm{x}$ & - & - & - & - & - & - & - & - & - & - & - & - & - \\
\hline Uvigerina hispida & - & $\mathrm{x}$ & - & - & - & - & - & - & - & - & - & - & - & - & - \\
\hline Uvigerinella californica & - & - & - & - & - & - & - & - & - & - & - & - & - & $\mathrm{x}$ & $\mathrm{x}$ \\
\hline Uvigerinella obesa & - & - & - & - & - & - & - & - & - & - & $\mathrm{x}$ & - & - & $\mathrm{x}$ & - \\
\hline Uvigerinella obesa impolita & - & - & - & - & - & - & $\mathrm{x}$ & - & - & - & - & - & - & - & - \\
\hline Uvigerinella spp. & - & - & - & - & - & - & - & - & - & $\mathrm{x}$ & - & - & - & - & - \\
\hline Valvulineria sp. & & - & & & & & & - & - & - & & & $x$ & & \\
\hline
\end{tabular}

Microfossil samples Mf7880, Mf7881, Mf7882, and Mf7883 are from an isolated outcrop of unit Tsm and removed from other samples in this unit. These samples contain a few arenaceous benthic foraminiferal species, which are primarily long-ranging Tertiary forms. The presence of Cyclammina pacifica implies an Eocene or younger age in California strata and lower middle bathyal depths (1,500-2,000 m). None of the other assemblages from unit Tsm contained similar faunas.

The remaining fossiliferous samples from unit Tsm range in age from Oligocene through middle Miocene. Sample Mf8095 is assigned to the Oligocene Zemorrian Stage based on a unique assemblage, which contains Cassidulina crassipunctata and Uvigerinella obesa impolita. This assemblage also suggests that deposition occurred at upper middle bathyal depths (500-1,500 m). Samples Mf805, Mf807, Mf837, Mf838, Mf882, and Mf883 contain benthic foraminiferal assemblages diagnostic of an early to middle Miocene age. These assemblages are assigned to the late Saucesian through Relizian Stages based on the ranges of Fursenkoina californiensis, Plectofrondicularia miocenica, P. miocenica directa, Siphogenerina branneri, S. transversa, Uvigerinella obesa, and U. obesa impolita. Lower bathyal depths are indicated for Mf805 but the other samples were deposited at upper bathyal (150-500 m) or upper middle bathyal (500-1,500 m) depths. 
Faunas from unit Tsm of Graymer and others (1994b, 1996) and Graymer (2000) range in age from early Eocene to middle Miocene. This age spans an interval with considerable biostratigraphic, ecologic, and tectonic changes and therefore, strata assigned to this unit may represent more than one unit. Location of the samples may be part of the problem, as many of the older samples were described without coordinates and the location was approximate. Several of the samples are located near fault boundaries or within fault zones so the sample may be a sliver of another unit. This appears to be the case for the Eocene samples (Mf8834, Mf8120, Mf8412, Mf7880, Mf7881, Mf7882, and Mf7883), which represent the early Eocene, late Eocene, and Eocene or younger, and have few to no species in common. The fauna in the Zemorrian Stage assemblage is not found in any other unit in the east San Francisco Bay region. The late Saucesian through Relizian Stage assemblages are well represented. Zemorrian and late Saucesian through Relizian Stage assemblages are located in the same general area of Block I and are assumed to represent the age of the Tsm unit.

No samples were collected from the overlying unnamed mudstone unit, Tush of Graymer and others (1994b, 1996), in Block I. Two samples (Mf802 and Mf803) were collected from rocks mapped as unnamed Cretaceous sedimentary rocks (unit Ku of Graymer and others, 1994b, 1996, and Graymer, 2000), in Block VII, across the Miller Creek Fault from unit Tush in Block I; these rocks probably represent a fault sliver of unit Tush. Field locations of these samples cannot be verified, but D. Radbruch (unpublished Examination and Report, 1963) believed the samples were from a Miocene unit. Benthic foraminifers present in this sample include several species that suggest a Miocene age and Siphogeneria hughesi, which restricts this assemblage to the middle Miocene Relizian Stage. None of the specimens are common to the underlying Tsm assemblages and represent deposition in upper bathyal biofacies $(150-500 \mathrm{~m})$.

Several samples were examined from the Claremont chert of Graymer (2000), which overlies unit Tush although the base is faulted (fig. 6). Most of the Claremont chert samples were barren or not diagnostic in age, containing either poorly preserved arenaceous species or long-ranging Miocene species such as Fursenkoina californiensis and Siphogenerina spp. (table 5). Sample Mf806 contains Siphogenerina hughesi, which restricts that sample to the early Relizian Stage. In addition, one sample each was taken from strata mapped as the Tice Shale (Mf8087), Orinda Formation (Mf1528), and Moraga Formation (Mf556) (Graymer, 2000). The foraminiferal assemblages from these samples indicate that they probably represent the Claremont chert. Sample Mf8087 contains only one species, Fursenkoina californiensis, which ranges throughout the Miocene; Mf1528 contains Valvulineria miocenica, which suggests a middle Miocene age and the Luisian Stage; and Mf556 contains Valvulineria californica, which indicates the middle Miocene Luisian Stage. Species with upper depth limits in the neritic biofacies are rare in these assemblages and downslope transport, if any, was from the upper slope only. Foraminiferal assemblages suggest deposition occurred at upper bathyal depths (150-500 m) based on the upper depth limits of the Fursenkoina, Siphogenerina, Uvigerina, and Valvulineria.

Sample Mf555 from the Claremont chert contains a fauna that is characteristic of the Mohnian Stage and is late middle to late Miocene in age. Age diagnostic species include Bolivina decurtata, Concavella gyoidinaformis, Epistominella capitanensis, and Fursenkoina californiensis grandis. The assemblage also suggests deposition occurred at upper middle bathyal depths (500-1,500 m). Because the age and bathymetry of Mf555 is different from the other Claremont chert assemblages and because this sample was taken near a fault, it may represent a younger unit such as the thin unit described by Jones and Curtis (1991) near Round Top, which lies between the Claremont chert and the Orinda Formation and is composed of a fine grained marine sandstone and conglomerate with clasts of 
Claremont chert. This Mohnian assemblage may also be part of a slightly younger, deeper water facies of the Claremont chert.

In Block I, the nonmarine Contra Costa Group discordantly overlies the Claremont chert and consists of the Orinda, Moraga, and Siesta Formations, and the Bald Peak Basalt. (Jones and Curtis, 1991; Wagner, 1978). No microfossil assemblages are known from these units except those discussed earlier which probably actually represent the Claremont chert.

The Orinda Formation, which is interpreted as nonmarine, contains vertebrate fossils assigned to the latest Barstovian to earliest Clarendonian North American land mammal (NALM) Stages (late middle to late Miocene in age). A basalt 100 feet above the base of the formation has been dated as 11.3 $\pm 1.4 \mathrm{Ma}$ (Lindquist and Morganthaler, 1991) and this age supports the late Miocene age. Dates from basalts in the overlying Moraga Formation (9.0 \pm 0.3 to 10.2 $\pm 0.5 \mathrm{Ma}$; Curtis, 1989) and Bald Peak Basalt $(8.37 \pm 0.2$ and $8.46 \pm 0.2 \mathrm{Ma}$; Curtis, 1989) as well as Clarendonian NALM Stage vertebrate fossils in the Siesta Formation further support the termination of marine sedimentation in the late middle or early late Miocene.

Table 5. Benthic foraminifers from the Claremont chert and rocks mapped as the Tice Shale (Tt), Orinda Formation (Tor), and Moraga Formation (Tms) in Block I.

[Taxonomy of species is given in the section entitled "Taxonomic Notes." $\mathrm{X}$ indicates the species is present; cf. indicates that the specimens resemble or compare to the species. Samples that are barren of microfossils include Mf7840, Mf7841, Mf7842, Mf8082, Mf8083, Mf8085, Mf8089, and Mf8090, all from Claremont chert; Mf8086 and Mf8088, Tice Shale; Mf3354, Oursan Sandstone]

\begin{tabular}{|c|c|c|c|c|c|c|c|c|c|c|c|c|}
\hline \multirow{2}{*}{$\begin{array}{l}\text { Benthic foraminifers from the } \\
\text { Claremont chert and rocks mapped } \\
\text { as the Tice Shale (Tt), Orinda } \\
\text { Formation (Tor), and Moraga } \\
\text { Formation (Tms) in Block I }\end{array}$} & \multicolumn{9}{|c|}{ Claremont chert } & \multirow{2}{*}{ 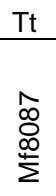 } & \multirow{2}{*}{$\begin{array}{l}\text { Tor } \\
\stackrel{\infty}{N} \\
\stackrel{\infty}{\infty} \\
\stackrel{\infty}{\Sigma}\end{array}$} & \multirow{2}{*}{ 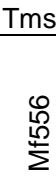 } \\
\hline & 員 & $\begin{array}{l}\stackrel{N}{2} \\
\stackrel{?}{\Sigma}\end{array}$ & 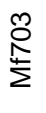 & $\begin{array}{l}0 \\
\stackrel{\infty}{\infty} \\
\stackrel{\infty}{\perp}\end{array}$ & 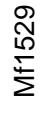 & $\begin{array}{l}\text { 离 } \\
\stackrel{0}{0} \\
\stackrel{1}{\perp}\end{array}$ & 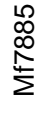 & $\begin{array}{l}\bar{\infty} \\
\infty \\
\infty \\
\sum^{\infty}\end{array}$ & 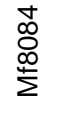 & & & \\
\hline Bolivina brevior & - & - & - & - & - & - & - & - & - & - & - & cf. \\
\hline Bolivina decurtata & $\mathrm{x}$ & - & - & - & - & - & - & - & - & - & - & - \\
\hline Bolivina marginata & - & - & - & - & - & - & - & - & - & - & - & - \\
\hline Bolivina salinasensis & - & - & - & $\mathrm{x}$ & - & - & - & - & - & - & - & - \\
\hline Bolivina spp. & - & $\mathrm{x}$ & - & - & $\mathrm{x}$ & - & - & - & - & - & - & - \\
\hline Bolivina vaughani & - & - & - & - & - & - & - & - & - & - & - & cf. \\
\hline Buliminella curta & - & - & - & $\mathrm{x}$ & - & - & - & - & - & - & - & - \\
\hline Buliminella subfusiformis & $\mathrm{x}$ & - & - & $\mathrm{x}$ & $\mathrm{x}$ & - & - & - & - & - & $\mathrm{x}$ & - \\
\hline Concavella gyroidinaformis & $\mathrm{x}$ & - & - & 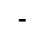 & - & 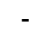 & - & - & - & - & - & - \\
\hline Cyclammina spp. & - & - & - & - & - & $\mathrm{x}$ & - & - & - & - & - & - \\
\hline Dentalina spp. & - & - & - & $\mathrm{x}$ & - & - & - & - & - & - & - & - \\
\hline Epistominella capitanensis & $\mathrm{x}$ & - & - & - & - & - & - & - & - & - & - & - \\
\hline Eponides spp. & - & $\mathrm{x}$ & - & - & - & - & - & - & - & - & - & - \\
\hline Fursenkoina californiensis & - & - & - & - & - & - & $\mathrm{x}$ & cf. & cf. & cf. & $\mathrm{x}$ & - \\
\hline Fursenkoina californiensis grandis & $\mathrm{x}$ & - & - & - & - & - & - & - & - & - & - & - \\
\hline Marginulina beali & - & - & - & - & - & - & - & - & - & - & - & - \\
\hline Nonionella costifera & - & - & - & - & $\mathrm{x}$ & - & - & - & - & - & - & - \\
\hline Nonionella spp. & $\mathrm{x}$ & - & - & - & - & - & - & - & - & - & - & - \\
\hline Siphogenerina hughesi & - & - & - & $\mathrm{x}$ & - & - & - & - & - & - & - & - \\
\hline Siphogenerina spp. & - & $\mathrm{x}$ & $x$ & - & - & - & - & - & - & - & - & - \\
\hline Uvigerina spp. & - & - & $\mathrm{x}$ & - & $x$ & - & - & - & - & - & - & $\mathrm{x}$ \\
\hline Valvulineria californica & - & - & - & - & 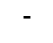 & - & - & - & - & - & - & $\mathrm{X}$ \\
\hline Valvulineria miocenica & - & - & - & - & - & - & - & - & - & - & $\mathrm{x}$ & - \\
\hline Valvulineria spp. & - & - & - & - & $\mathrm{X}$ & - & - & - & - & - & - & - \\
\hline
\end{tabular}




\section{Block II}

Block II is bounded to the west by Block III and the Pinole, Moraga, Palomares, and Storybrook Faults; and bounded to the east by the Franklin and Calaveras Faults (fig. 1). Block II, as defined herein, corresponds to a portion of the East Bay Hills block of Buising and Walker (1995) and most of the Lafayette block of Graham and others (1984). The only Paleogene unit present is unit Tshc of Graymer and others (1994b, 1996) which unconformably overlies the Cretaceous Great Valley sequence and underlies the Neogene units. Although the Neogene section in Block II includes many units (figs. 7, 8), only the San Ramon Sandstone, Sobrante Sandstone, Claremont Shale, Oursan Sandstone, Tice Shale, Hambre Sandstone, Rodeo Shale, and Briones Sandstone were sampled. Samples from the Tertiary section were collected in three geographic areas: northern, central near the San Pablo and Briones reservoirs, and southern near Dublin. These areas are discussed separately. Samples Mf3265, Mf3266, Mf3267, from the middle of Block II, do not contain diagnostic microfossils or are barren of microfossils.

\section{Northern Part of Block II}

Several samples were collected from the northern part of Block II in a unit mapped as Qpaf (alluvial fan and stream deposits; Graymer, 2000), but most likely represent the unnamed Paleogene unit Tshc (fig. 7). All five samples (table 6) are located adjacent to Block III and probably are within the fault zone. The location of the samples in a unit identified as Qpaf and in an area surrounded by Miocene units indicates that the samples were taken in fault slivers of the unnamed unit Tshc. The nearest exposure of unit Tshc is south of the sample localities.

Both Mf1099 samples (2N/3W-27B3 and 2N/3W-27B4) are assigned to the early Eocene Penutian Stage based on the appearance of Bulimina macilenta, Cibicidoides eocenicus, C. praemundulus, Uvigerina lodoensis mirmirae, and Vaginulopsis asperuliformis (table 6). Additionally, the presence of Bulimina callahani, Silicosigmoilina californica, and Uvigerina lodoensis mirmarae suggest that the assemblage is coeval with planktic foraminiferal zones P7 through P9. Although samples Mf1085B, Mf1097, and Mf1098 contain many of the same species as Mf1099 (field numbers 2N/3W-27B3 and 2N/3W-27B4), the presence of Cibicidoides subspiratus, Eponides mexicanus, Lenticulina cf. L. welchi, and Siphonia wilcoxensis, suggest that these assemblages are middle Eocene (Ulatisian) in age, coeval with planktic foraminiferal zones late P9 through P10. Sample Mf1085A contains only two long-ranging Eocene species and provides little additional information to the age interpretation. Deposition occurred at lower bathyal to abyssal depths $(\geq 2,000 \mathrm{~m})$. 


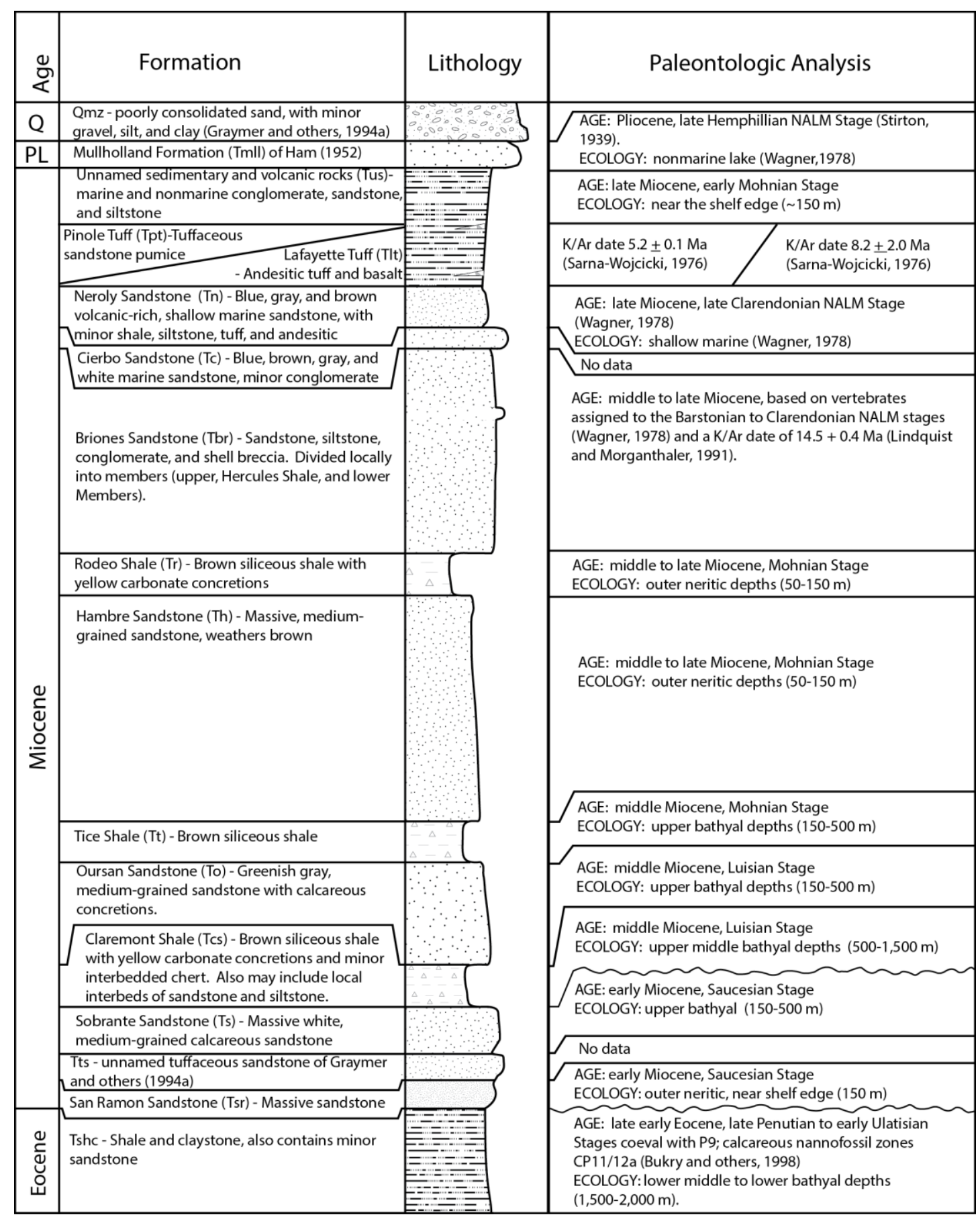

Figure 7. Composite columnar section for Block II, northern part and San Pablo and Briones Reservoirs. Age and environmental interpretations are summarized from the text. Montezuma Formation (Qmz of Graymer and others, 1994a) considered Pleistocene alluvium by Helley and Graymer (1997) and Blake and others (2000). Q, Quaternary; PL, Pliocene. 


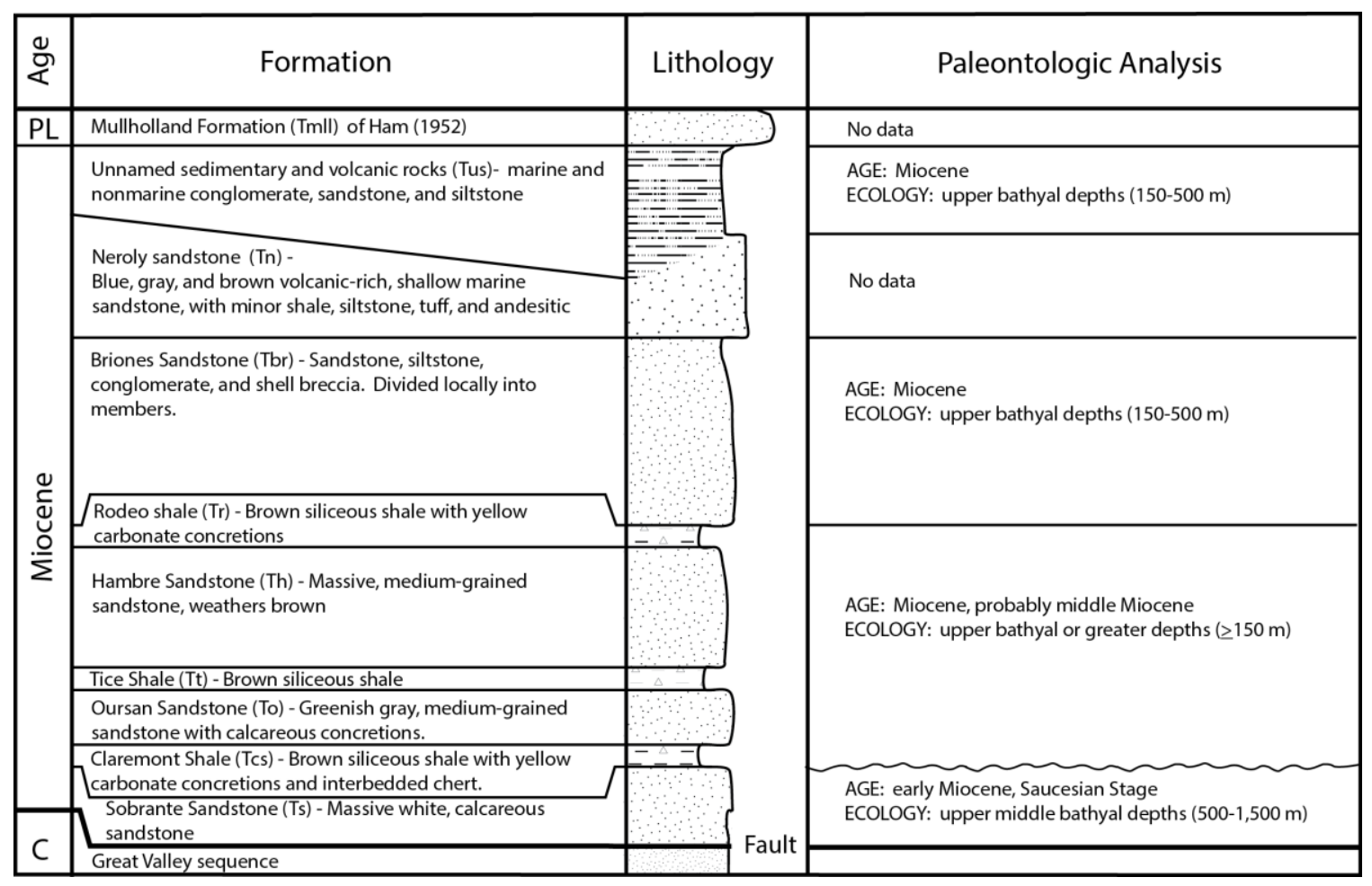

Figure 8. Composite columnar section for Block II, Dublin. Age and environmental interpretations are summarized from the text. Q, Quaternary; PL, Pliocene; C, Cretaceous.

Table 6. Benthic foraminifers from unit Tshc of Graymer and others $(1994 a, 1996)$ in the northern part of Block II. [Taxonomy of species is given in the section entitled "Taxonomic Notes." $\mathrm{X}$ indicates the species is present; cf. indicates that the specimens resemble or compare to the species]

\begin{tabular}{|c|c|c|c|c|c|}
\hline $\begin{array}{c}\text { Benthic foraminifers in unit } \\
\text { Tshc of Graymer and others } \\
\text { (1994a, 1996) in northern } \\
\text { Block II }\end{array}$ & 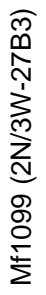 & 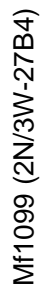 & 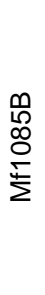 & $\begin{array}{l}\text { के } \\
\text { Oे } \\
\frac{1}{\Sigma}\end{array}$ & 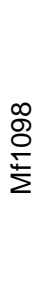 \\
\hline Alabamina wilcoxensis & $x$ & $x$ & - & - & - \\
\hline Allomorphina conica & $\mathrm{X}$ & - & - & - & - \\
\hline Ammobacculites spp. & $X$ & - & - & - & - \\
\hline Ammodiscus incertus & $\mathrm{X}$ & $\mathrm{X}$ & - & - & - \\
\hline Amphimorphina spp. & - & $x$ & - & - & - \\
\hline Anomalinoides capitatus & $X$ & $X$ & $X$ & - & $X$ \\
\hline Anomalina regina & $x$ & $x$ & $x$ & $X$ & - \\
\hline Aragonia aragonensis & - & - & $\mathrm{X}$ & - & - \\
\hline Bathysiphon santecruis & $x$ & $x$ & - & - & - \\
\hline Bathysiphon spp. & $X$ & $X$ & $X$ & - & $X$ \\
\hline Bifarina eleganta & $X$ & - & - & - & - \\
\hline Bolivina explicata lodoensis & - & $X$ & - & $X$ & - \\
\hline
\end{tabular}

\begin{tabular}{|c|c|c|c|c|c|}
\hline $\begin{array}{c}\text { Benthic foraminifers in unit } \\
\text { Tshc of Graymer and others } \\
\text { (1994a, 1996) in northern } \\
\text { Block II }\end{array}$ & 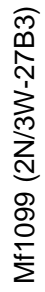 & 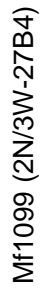 & $\begin{array}{l}\mathscr{M} \\
\mathscr{L} \\
0 \\
0 \\
\sum^{+}\end{array}$ & $\begin{array}{l}\text { 용 } \\
\text { 올 }\end{array}$ & $\begin{array}{l}\stackrel{\infty}{\circ} \\
\frac{0}{0} \\
\frac{1}{\Sigma}\end{array}$ \\
\hline Bulimina alazaensis & - & - & $x$ & - & - \\
\hline Bulimina callahani & $\mathrm{X}$ & $X$ & - & - & $X$ \\
\hline Bulimina macilenta & $X$ & $X$ & $X$ & - & $X$ \\
\hline Bulimina trinitatensis & - & $\mathrm{X}$ & - & - & - \\
\hline Bulimina tuxapamensis & - & - & $X$ & - & - \\
\hline Buliminella robertsi & $\mathrm{X}$ & $\mathrm{X}$ & - & - & $X$ \\
\hline Chilostomella cylindroides & - & $X$ & - & - & - \\
\hline Chrysalongonium elongatum & $X$ & $X$ & - & - & $X$ \\
\hline Chrysalongonium laeve & $X$ & $X$ & - & - & - \\
\hline Chrysalongonium tenuicostatum & $\mathrm{X}$ & $\mathrm{X}$ & $\mathrm{X}$ & - & - \\
\hline Cibicides felix & - & - & - & $X$ & - \\
\hline Cibicides fortunatus & - & - & - & $\mathrm{X}$ & - \\
\hline
\end{tabular}




\begin{tabular}{|c|c|c|c|c|c|}
\hline $\begin{array}{c}\text { Benthic foraminifers in unit } \\
\text { Tshc of Graymer and others } \\
(1994 a, 1996) \text { in northern } \\
\text { Block II }\end{array}$ & 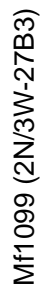 & 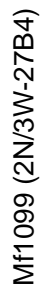 & $\begin{array}{l}\infty \\
\infty \\
\infty \\
\infty \\
\stackrel{+}{\Sigma}\end{array}$ & $\begin{array}{l}\hat{O} \\
\text { O } \\
\frac{1}{\Sigma}\end{array}$ & $\begin{array}{l}\infty \\
\text { o } \\
\stackrel{\Xi}{\Sigma}\end{array}$ \\
\hline Cibicidoides eocaenus & $x$ & $x$ & $\mathrm{X}$ & $\mathrm{X}$ & $\mathrm{X}$ \\
\hline Cibicidoides eponidiformis & - & $x$ & - & - & - \\
\hline Cibicidoides pachecoensis & - & $x$ & - & - & - \\
\hline Cibicidoides praemundulus & $x$ & $x$ & - & - & - \\
\hline Cibicidoides subspiratus & - & - & $\mathrm{X}$ & $\mathrm{X}$ & $x$ \\
\hline Cibicidoides spp. & $x$ & $x$ & - & - & $\mathrm{X}$ \\
\hline Cibicidoides venzuelanus & - & $x$ & $\mathrm{X}$ & - & - \\
\hline Clavulina anglica & - & $x$ & - & - & $\mathrm{X}$ \\
\hline Clavulinoides californicus & - & $x$ & - & - & - \\
\hline Cyclammina pacifica & - & $x$ & - & - & - \\
\hline Cyclammina simiensis & $x$ & - & $\mathrm{X}$ & - & $\mathrm{x}$ \\
\hline Dentalina approximata & - & - & - & $x$ & - \\
\hline Dentalina catenula & $\mathrm{X}$ & $x$ & - & - & - \\
\hline Dentalina colei & $x$ & $x$ & - & $x$ & - \\
\hline Dentalina communis & $x$ & - & - & - & - \\
\hline Dentalina globulicauda & $x$ & - & $x$ & - & - \\
\hline Dorothia principiensis & - & $x$ & $\mathrm{X}$ & - & $x$ \\
\hline Eponides lodoensis & - & $x$ & - & - & - \\
\hline Eponides mexicanus & - & - & - & $?$ & - \\
\hline Fissurina marginata & $x$ & $x$ & - & - & - \\
\hline Gaudryina laevigata & $x$ & $x$ & - & $x$ & $x$ \\
\hline Glandulina laevigata & $x$ & $x$ & - & - & - \\
\hline Globocassidulina globosa & $\mathrm{x}$ & - & - & - & $x$ \\
\hline Globulina spp. & $x$ & $x$ & - & - & - \\
\hline Glomospira charoides & $x$ & - & - & - & - \\
\hline Guttulina problema & $x$ & - & - & - & - \\
\hline Gyroidina octocameratus & - & $x$ & - & - & - \\
\hline Gyroidina soldanii & $x$ & $x$ & - & - & - \\
\hline Haplophragmoides eggeri & $x$ & $x$ & $\mathrm{X}$ & - & $x$ \\
\hline Karreriella conversa & $x$ & $x$ & - & - & $x$ \\
\hline Karreriella elongata & $x$ & $x$ & - & - & - \\
\hline Lagena becki & - & $x$ & - & - & - \\
\hline Lagena costata & $x$ & - & - & - & - \\
\hline Lagena spp. & $\mathrm{X}$ & - & $\mathrm{X}$ & - & - \\
\hline Lagena vulgaris & $x$ & $x$ & - & - & - \\
\hline Lenticulina midwayensis & - & - & - & $X$ & $\mathrm{X}$ \\
\hline Lenticulina pseudovortex & - & - & - & $\mathrm{X}$ & $\mathrm{x}$ \\
\hline Lenticulina spp. & $x$ & $x$ & $\mathrm{X}$ & - & $\mathrm{X}$ \\
\hline Lenticulina welchi & - & - & - & cf. & - \\
\hline Lituotuba lituiformis & $\mathrm{x}$ & - & - & - & $x$ \\
\hline
\end{tabular}

\begin{tabular}{|c|c|c|c|c|c|}
\hline $\begin{array}{c}\text { Benthic foraminifers in unit } \\
\text { Tshc of Graymer and others } \\
(1994 a, 1996) \text { in northern } \\
\text { Block II }\end{array}$ & 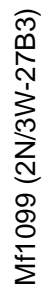 & 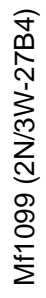 & $\begin{array}{l}\infty \\
10 \\
\infty \\
0 \\
0 \\
\sum\end{array}$ & $\begin{array}{l}\hat{\circ} \\
\frac{1}{2} \\
\frac{1}{\Sigma}\end{array}$ & $\begin{array}{l}\infty \\
\text { ᄋ } \\
\text { ○ } \\
\frac{ \pm}{\Sigma}\end{array}$ \\
\hline Marginulina adunca & $x$ & - & - & - & - \\
\hline Marginulina exima & $x$ & $x$ & - & - & - \\
\hline Marginulina spp. & $x$ & $x$ & - & - & - \\
\hline Marginulina subbullata & $x$ & $x$ & - & $x$ & - \\
\hline Nonionella ansata & $x$ & - & - & - & - \\
\hline Nodosaria delicata & - & - & - & - & $x$ \\
\hline Nodosaria latejugata & - & $x$ & $x$ & $x$ & - \\
\hline Nodosaria longiscata & - & $x$ & $x$ & - & $x$ \\
\hline Nodosaria spp. & - & $x$ & - & - & - \\
\hline Nodosarella advena & - & $x$ & - & - & - \\
\hline Nodosarella constricta & $x$ & - & - & - & - \\
\hline Nuttaloides truempyi & $x$ & $x$ & $x$ & - & $x$ \\
\hline Oridorsalis umbonatus & $x$ & $x$ & $x$ & - & - \\
\hline Osangularia mexicana & $x$ & $x$ & - & $x$ & $x$ \\
\hline Pleurostomella nuttalli & $x$ & - & - & - & - \\
\hline Pseudonodosaria inflata & $x$ & $x$ & - & - & - \\
\hline Pullenia eocenica & $x$ & $x$ & - & - & $x$ \\
\hline Pullenia spp. & $x$ & - & - & - & - \\
\hline Pyrulina cylindroides & $x$ & $x$ & - & - & - \\
\hline Saracenaria spp. & $x$ & - & - & - & $x$ \\
\hline Silicosigmoilina californica & $x$ & $x$ & - & - & - \\
\hline Siphonia wilcoxensis & - & - & - & $x$ & - \\
\hline Spiroloculina texana & - & $x$ & - & - & - \\
\hline Spiroplectammina directa & - & - & $x$ & - & - \\
\hline Spiroplectammina richardi & $X$ & $X$ & - & - & - \\
\hline Stilostomella adolphina & - & $x$ & - & - & - \\
\hline Stilostomella lepidula & $x$ & $x$ & - & - & $\mathrm{x}$ \\
\hline Textularia adalta & $x$ & - & - & - & - \\
\hline Trifarina advena californica & $x$ & $x$ & - & - & - \\
\hline Tritaxilina colei & $x$ & $x$ & - & - & - \\
\hline Trochammina globigeriniformis & $x$ & $x$ & $x$ & - & - \\
\hline Uvigerina alabamensis & - & - & - & cf. & - \\
\hline Uvigerina hispida & cf. & cf. & - & - & - \\
\hline Uvigerina lodoensis miriamae & $x$ & $x$ & - & - & - \\
\hline Vaginulinopsis asperuliformis & $x$ & $x$ & $x$ & $x$ & $\mathrm{X}$ \\
\hline Vaginulinopsis nudicostata & - & - & - & $x$ & - \\
\hline Verneuilina triangulata & $x$ & $x$ & - & - & - \\
\hline Vulvulina curta & $\mathrm{x}$ & $\mathrm{x}$ & - & - & - \\
\hline
\end{tabular}




\section{San Pablo and Briones Reservoirs}

The Tertiary section near the San Pablo and Briones Reservoirs is composed of an unnamed Paleogene unit (Tshc) and Neogene units that unconformably overlie the Paleogene and Cretaceous units. The unnamed Paleogene unit (Tshc), Sobrante Sandstone, Claremont Shale, Oursan Sandstone, Tice Shale, Hambre Sandstone, Rodeo Shale, and unnamed sedimentary and volcanic rocks (Tus) were sampled for microfossils, whereas no new microfossils samples were taken from the unnamed tuffaceous sandstone (Tts), San Ramon Sandstone, Briones Sandstone, Cierbo Sandstone, Neroly Sandstone, and the overlying nonmarine strata and tuffs (fig. 7).

Unit Tshc of Graymer and others (1994b) was sampled just north of San Pablo Reservoir (Mf1095, Mf8030, Mf8389 to Mf8402) (table 7) and just north of Briones Reservoir (Mf7813 to Mf7816, and Mf7825) (table 8). This unit contains similar benthic foraminiferal assemblages in both locations, which are late early Eocene in age and assigned to the late Penutian and early Ulatisian Stages, coeval with planktic foraminiferal zone P9. The early Eocene age of unit Tshc is constrained by the last appearance of Allomorphina conica, Bolivina kleinpelli, Hanzawaia mauricensis, Lenticulina cf. L. welchi, and Loxostomoides applinae; the first appearance Cibicidoides grimsdalei, C. subspiratus, Karreriella elongata, Plectina garzaensis, Pullenia eocenica, Plectofrondicularia paucicostata, and Vaginulinopsis saundersi; and the range of Bolivina excavata, and Uvigerina lodoensis miriamae. The presence of arenaceous foraminifers such as Hormosina sp., Lituituba lituiformis, Silicosigmoilina californica, Spiroplectammina directa, and Trochamminoides contortus suggest an early Eocene age whereas the presence of Eggerella elongata implies middle Eocene. Although not restricted, the presence of Hoeglundina eocenica, Siphonia wilcoxensis, and Elphidium californicum also support a middle rather than an early Eocene age. This mix of bathyal early Eocene species and neritic to upper bathyal middle Eocene species is characteristic of the sea level change and reworking of faunas in planktic foraminiferal zone P9. Calcareous nannofossils present in many of the samples in this unit are assigned to the early to middle Eocene zones CP11/12a, undifferentiated (Bukry and others, 1998), which supports the middle Eocene age suggested by the benthic foraminifers. 
Table 7. Benthic foraminifers from unit Tshc of Graymer and others (1994a, 1996) near San Pablo Reservoir in Block II.

[Taxonomy of species is given in the section entitled "Taxonomic Notes." $\mathrm{X}$ indicates the species is present, cf. indicates that the specimens resemble or compare to the species]

\begin{tabular}{|c|c|c|c|c|c|c|c|c|c|c|c|c|c|c|c|c|}
\hline $\begin{array}{c}\text { Benthic foraminifers from unit Tshc } \\
\text { of Graymer and others } \\
\text { (1994a,1996), near San Pablo } \\
\text { Reservoir in Block II }\end{array}$ & $\begin{array}{l}\text { 용 } \\
\text { O } \\
\frac{1}{2}\end{array}$ & 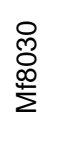 & $\begin{array}{l}\mathscr{P} \\
\infty \\
\infty \\
\stackrel{\infty}{\Sigma}\end{array}$ & 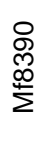 & $\begin{array}{l}\bar{\infty} \\
\infty \\
\infty \\
\Sigma\end{array}$ & $\begin{array}{l}\text { N } \\
\text { ্ֻ } \\
\stackrel{\infty}{\Sigma}\end{array}$ & $\begin{array}{l}\text { ల్ల } \\
\stackrel{\infty}{\infty} \\
\stackrel{\infty}{\Sigma}\end{array}$ & 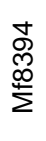 & 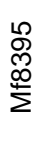 & 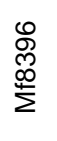 & 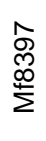 & $\begin{array}{l}\infty \\
\stackrel{\infty}{\infty} \\
\infty \\
\Sigma \\
\Sigma\end{array}$ & 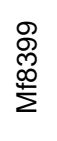 & 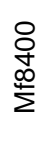 & 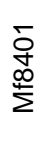 & 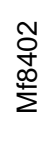 \\
\hline Alabamina wilcoxensis & - & - & - & - & - & - & - & - & - & - & - & - & - & $x$ & - & - \\
\hline Allomorphina conica & $x$ & - & - & - & - & - & - & - & - & - & $x$ & - & - & - & - & - \\
\hline Allomorphina spp. & - & - & - & - & - & - & - & - & - & - & - & - & - & - & $x$ & - \\
\hline Ammobacculites spp. & - & - & - & - & - & - & - & - & - & - & - & - & - & - & - & - \\
\hline Ammodiscus incertus & $x$ & - & - & $x$ & - & - & - & - & - & - & - & $x$ & - & $x$ & - & $X$ \\
\hline Anomalinoides capitatus & - & $X$ & - & $x$ & - & - & - & - & - & - & - & - & - & - & - & - \\
\hline Anomalinoides semicribratus & $x$ & - & - & - & - & - & - & - & - & - & - & - & - & - & - & - \\
\hline Anomalina keenae & - & - & - & $x$ & - & - & - & $x$ & - & - & - & - & - & - & - & - \\
\hline Anomalina regina & $x$ & $\mathrm{X}$ & $x$ & - & $\mathrm{x}$ & - & $x$ & $x$ & $x$ & $x$ & - & - & $\mathrm{X}$ & $x$ & - & - \\
\hline Aragonia aragonensis & $x$ & - & - & - & - & - & - & - & - & - & - & - & - & - & - & - \\
\hline Bathysiphon eocenicus & - & $\mathrm{X}$ & $x$ & $x$ & - & - & $x$ & $x$ & - & $x$ & - & $x$ & $X$ & $x$ & $x$ & $\mathrm{X}$ \\
\hline Bathysiphon santecruis & $\mathrm{X}$ & - & $x$ & $x$ & - & - & $x$ & $x$ & $x$ & - & - & - & $\mathrm{X}$ & $x$ & - & $X$ \\
\hline Bathysiphon spp. & $x$ & - & - & $x$ & $x$ & $x$ & - & $x$ & - & $x$ & $x$ & $\mathrm{X}$ & - & - & $x$ & - \\
\hline Bolivina kleinpelli & - & - & - & - & - & - & $x$ & $x$ & - & - & - & - & - & - & - & - \\
\hline Bolivina spp. & - & $x$ & - & - & - & - & - & - & - & - & - & - & - & - & - & - \\
\hline Bulimina excavata & $x$ & - & - & - & - & - & - & - & - & - & - & - & - & - & - & - \\
\hline Bulimina macilenta & $X$ & - & - & - & - & - & - & - & - & - & $x$ & - & - & - & - & - \\
\hline Bulimina trinitatensis & $x$ & - & - & - & - & - & - & - & - & - & - & - & - & - & - & - \\
\hline Buliminella robertsi & $x$ & - & - & - & - & - & - & - & - & - & - & - & - & - & - & - \\
\hline Chrysalongonium elongatum & $x$ & - & - & - & - & - & - & $x$ & - & $x$ & - & $x$ & - & - & - & - \\
\hline Chrysalongonium laeve & $X$ & - & - & - & - & - & - & - & - & - & - & - & - & - & - & - \\
\hline Chrysalongonium spp. & $x$ & - & - & - & - & - & $x$ & - & - & - & - & - & - & - & - & - \\
\hline Chrysalongonium teniucostatum & $x$ & - & $x$ & - & - & - & - & - & - & - & - & - & - & - & - & - \\
\hline Cibicides martinezensis & $x$ & - & - & - & - & - & - & - & - & - & - & - & - & - & - & - \\
\hline Cibicidoides cocoaensis & $x$ & - & - & - & - & - & - & - & - & - & - & - & - & - & - & - \\
\hline Cibicidoides eocaenus & - & $x$ & - & $x$ & $x$ & $x$ & $x$ & $x$ & $x$ & $x$ & $x$ & $x$ & - & $x$ & - & $x$ \\
\hline Cibicidoides eponidiformis & $x$ & - & - & - & $x$ & - & $x$ & - & - & - & $x$ & - & - & - & - & - \\
\hline Cibicidoides grimsdalei & - & - & - & - & $x$ & - & - & - & - & - & - & - & $\mathrm{X}$ & $x$ & $x$ & $\mathrm{X}$ \\
\hline Cibicidoides praemundulus & $\mathrm{X}$ & - & - & - & - & - & - & - & - & - & - & - & - & - & - & - \\
\hline Cibicidoides subspiratus & - & - & $x$ & $x$ & $x$ & - & - & $x$ & - & - & - & $x$ & - & - & - & - \\
\hline Cibicidoides spp. & - & - & - & - & $\mathrm{X}$ & $x$ & $x$ & $X$ & $x$ & - & - & - & - & $x$ & - & $\mathrm{X}$ \\
\hline Cibicidoides venezuelanus & - & $\mathrm{X}$ & - & $x$ & $x$ & $x$ & $x$ & - & $x$ & $x$ & $x$ & $x$ & - & - & $x$ & - \\
\hline Clavulina anglica & $x$ & - & - & - & - & - & - & - & - & - & - & - & - & - & - & - \\
\hline Cyclammina simiensis & $x$ & - & - & - & - & - & - & - & - & - & - & - & - & - & - & - \\
\hline Dentalina colei & $x$ & - & $x$ & - & - & - & - & - & - & - & $x$ & $x$ & $\mathrm{X}$ & $x$ & $x$ & - \\
\hline Dentalina communis & $x$ & - & - & - & - & - & $x$ & - & $x$ & - & $x$ & - & - & - & - & - \\
\hline Dentalina consobrina & - & $\mathrm{X}$ & - & - & $x$ & - & - & $x$ & - & - & $x$ & $x$ & - & $x$ & - & - \\
\hline Dentalina globulicauda & $x$ & - & - & - & - & - & - & - & - & - & - & - & - & - & - & - \\
\hline Dentalina intorta & $x$ & - & - & - & - & - & - & - & - & - & - & - & - & - & - & - \\
\hline Dentalina spp. & - & - & - & - & $x$ & - & - & $x$ & $x$ & $x$ & $x$ & $x$ & $\mathrm{X}$ & $x$ & - & $X$ \\
\hline Dorothia bulletta & $x$ & - & - & - & - & - & - & - & - & - & - & - & - & - & - & - \\
\hline Dorothia principiensis & - & - & - & - & $x$ & - & - & - & - & - & - & - & - & - & $x$ & - \\
\hline Dorothia spp. & - & - & - & - & - & - & $x$ & - & - & - & - & $x$ & - & - & - & - \\
\hline Ellipsoglandulina labiata & $\mathrm{X}$ & - & - & - & - & - & - & - & - & - & - & - & - & - & - & - \\
\hline Elphidium californicum & - & - & - & - & - & - & - & - & $\mathrm{x}$ & - & - & - & - & - & - & - \\
\hline Eponides lodoensis & - & - & - & - & - & - & - & $X$ & - & - & - & - & - & $x$ & - & $\mathrm{X}$ \\
\hline Glandulina laevigata & $\mathrm{X}$ & - & - & - & - & - & - & - & - & - & - & - & - & - & - & - \\
\hline
\end{tabular}




\begin{tabular}{|c|c|c|c|c|c|c|c|c|c|c|c|c|c|c|c|c|}
\hline $\begin{array}{c}\text { Benthic foraminifers from unit Tshc } \\
\text { of Graymer and others } \\
\text { (1994a,1996), near San Pablo } \\
\text { Reservoir in Block II }\end{array}$ & $\begin{array}{l}\text { Lᄋ } \\
\text { O } \\
\frac{+}{2}\end{array}$ & 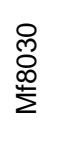 & $\begin{array}{l}\infty \\
\infty \\
\infty \\
\infty \\
\infty\end{array}$ & $\begin{array}{l}\text { ㅇ } \\
\text { ల } \\
\stackrel{\infty}{\Sigma}\end{array}$ & $\begin{array}{l}\bar{\infty} \\
\infty \\
\infty \\
\Sigma\end{array}$ & $\begin{array}{l}\text { N } \\
\text { } \\
\infty \\
\Sigma \\
\Sigma\end{array}$ & $\begin{array}{l}\text { ळ్ } \\
\stackrel{\infty}{\infty} \\
\stackrel{\infty}{\Sigma}\end{array}$ & $\begin{array}{l}\text { D } \\
\text { ల } \\
\infty \\
\Sigma\end{array}$ & $\begin{array}{l}\stackrel{L}{\infty} \\
\stackrel{\infty}{\infty} \\
\stackrel{\infty}{\Sigma}\end{array}$ & $\begin{array}{l}\mathscr{D} \\
\stackrel{\infty}{\infty} \\
\stackrel{\infty}{\Sigma}\end{array}$ & $\begin{array}{l}\hat{\infty} \\
\text { D } \\
\infty \\
\Sigma\end{array}$ & $\begin{array}{l}\infty \\
\text { O } \\
\substack{\infty \\
\Sigma}\end{array}$ & $\begin{array}{l}\text { D } \\
\text { O } \\
\stackrel{\infty}{\Sigma}\end{array}$ & 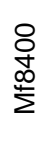 & $\begin{array}{l}\overline{0} \\
\text { + } \\
\infty \\
\Sigma\end{array}$ & $\begin{array}{l}\mathcal{N} \\
\stackrel{+}{+} \\
\infty \\
\stackrel{\infty}{\Sigma}\end{array}$ \\
\hline Globocassidulina globosa & $x$ & - & - & - & - & - & - & $x$ & - & - & - & - & - & $x$ & - & - \\
\hline Glomospira charoides & $x$ & - & - & - & - & - & - & - & - & - & - & - & - & - & - & - \\
\hline Guttulina spp. & - & - & - & - & - & - & - & - & $x$ & - & - & - & - & - & - & - \\
\hline Gyroidina octocameratus & $x$ & - & - & - & - & - & - & - & - & - & - & - & - & - & - & - \\
\hline Gyroidina soldanii & $x$ & - & - & - & - & - & - & $x$ & - & - & - & - & - & $x$ & - & $\mathrm{X}$ \\
\hline Gyroidina spp. & - & - & - & - & $x$ & - & - & - & - & - & - & - & - & - & - & - \\
\hline Hanzawaia mauricensis & - & - & - & - & $x$ & $x$ & - & $x$ & $x$ & - & $x$ & - & - & - & - & - \\
\hline Hanzawaia spp. & - & $\mathrm{X}$ & - & - & - & - & - & $x$ & - & - & - & - & - & $x$ & - & - \\
\hline Haplophragmoides eggeri & $x$ & - & - & $x$ & - & - & - & $x$ & - & - & - & - & - & $x$ & $x$ & $\mathrm{X}$ \\
\hline Haplophragmoides glabra & $x$ & - & - & - & - & - & - & - & - & - & - & - & - & - & - & - \\
\hline Haplophragmoides spp. & - & $\mathrm{X}$ & $x$ & - & - & $x$ & - & - & $x$ & $x$ & - & $\mathrm{X}$ & $X$ & - & - & - \\
\hline Hoeglundina eocenica & - & - & - & - & $x$ & - & $x$ & $x$ & - & - & $x$ & $x$ & $\mathrm{X}$ & - & - & $\mathrm{X}$ \\
\hline Hormosina spp. & $\mathrm{X}$ & - & - & - & - & - & - & - & - & - & - & - & - & - & - & - \\
\hline Karreriella horrida & - & - & $x$ & - & - & - & - & $x$ & - & $x$ & - & - & - & - & - & - \\
\hline Lagena spp. & - & - & - & - & $x$ & - & - & - & - & - & - & - & - & - & - & - \\
\hline Lagena vulgaris & $\mathrm{X}$ & - & - & - & - & - & - & - & - & - & - & - & - & - & - & - \\
\hline Lenticulina caritae & $\mathrm{X}$ & - & - & - & - & - & - & - & - & - & - & - & - & - & - & - \\
\hline Lenticulina chambersi & $\mathrm{X}$ & - & - & - & - & - & - & - & - & - & - & - & - & - & - & - \\
\hline Lenticulina limbosa hockleyensis & $\mathrm{X}$ & - & - & - & - & - & - & - & - & - & - & - & - & - & - & - \\
\hline Lenticulina pseudocultratus & $\mathrm{X}$ & - & - & - & - & - & - & - & - & - & - & - & - & - & - & - \\
\hline Lenticulina pseudovortex & $\mathrm{X}$ & - & - & - & - & - & - & - & - & - & - & - & - & - & - & - \\
\hline Lenticulina spp. & $x$ & $X$ & $x$ & $x$ & $x$ & $x$ & $x$ & $x$ & $x$ & $x$ & $x$ & $x$ & $X$ & $x$ & $x$ & $\mathrm{X}$ \\
\hline Lenticulina turbinatus & $\mathrm{X}$ & - & - & - & - & - & - & - & - & - & - & - & - & - & - & - \\
\hline Lenticulina vortex & $x$ & - & - & - & - & - & - & - & - & - & - & - & - & - & - & - \\
\hline Lenticulina welchi & - & - & - & - & - & - & - & - & - & - & cf. & - & - & - & - & - \\
\hline Lituotuba lituiformis & $x$ & - & - & - & - & - & - & - & - & - & - & - & - & - & - & - \\
\hline Loxostomoides applinae & - & - & - & - & - & - & - & - & - & - & - & $x$ & - & $x$ & $x$ & - \\
\hline Marginulina subbullata & $x$ & - & - & - & - & - & - & - & - & - & $x$ & - & - & - & $x$ & - \\
\hline Marginulina spp. & - & - & - & - & - & - & - & - & - & - & $x$ & - & - & - & $x$ & - \\
\hline Martinottiella spp. & - & - & - & - & - & - & - & - & $x$ & - & - & - & - & - & - & - \\
\hline Nonionella ansata & $\mathrm{X}$ & - & - & - & - & - & - & - & - & - & - & - & - & - & - & - \\
\hline Nodosaria deliciae & - & $X$ & - & - & $x$ & - & - & - & - & - & $x$ & - & - & $x$ & - & - \\
\hline Nodosaria latejugata & - & - & $x$ & - & - & - & $x$ & $x$ & $x$ & - & - & - & - & - & - & $\mathrm{X}$ \\
\hline Nodosaria longiscata & - & $\mathrm{X}$ & $x$ & - & $x$ & - & $x$ & $x$ & $x$ & - & $x$ & - & $\mathrm{X}$ & $x$ & - & $\mathrm{X}$ \\
\hline Nodosaria pyrula & - & - & $x$ & - & - & - & - & - & - & $x$ & - & - & - & - & - & - \\
\hline Nodosaria spp. & - & $\mathrm{X}$ & $x$ & - & $x$ & - & $x$ & - & - & $x$ & - & - & - & $x$ & $x$ & $\mathrm{X}$ \\
\hline Nodosarella atlantisae hispidula & - & - & - & - & - & - & - & - & $\mathrm{x}$ & - & - & $\mathrm{X}$ & $X$ & - & - & $X$ \\
\hline Nodosarella constricta & $\mathrm{x}$ & - & - & - & - & - & - & - & - & - & - & - & - & - & - & - \\
\hline Nuttaloides truempyi & $\mathrm{X}$ & - & - & $x$ & - & $x$ & - & $x$ & - & - & $x$ & - & $X$ & - & - & - \\
\hline Oridorsalis umbonatus & $x$ & - & - & - & - & - & - & $x$ & - & - & - & - & - & $x$ & - & $\mathrm{X}$ \\
\hline Osangularia mexicana & $x$ & $\mathrm{X}$ & - & - & $x$ & $x$ & $x$ & $x$ & $x$ & $x$ & $x$ & $\mathrm{X}$ & $\mathrm{X}$ & $x$ & $x$ & $\mathrm{X}$ \\
\hline Plectofrondicularia paucicostata & - & - & - & - & - & - & - & $x$ & - & - & - & - & - & - & - & - \\
\hline Pleurostomella nuttalli & $X$ & - & - & - & - & - & - & - & - & - & - & - & - & - & - & - \\
\hline Pleurostomella spp. & - & - & - & $x$ & - & - & - & - & - & - & - & - & - & - & - & - \\
\hline Pseudonodosaria conica & - & - & - & - & - & - & $x$ & - & - & - & - & - & - & - & $x$ & - \\
\hline Pseudonodosaria inflata & - & - & - & - & - & - & - & - & - & - & - & - & $\mathrm{X}$ & - & $x$ & $\mathrm{X}$ \\
\hline Pseudonodosaria spp. & - & - & - & - & $x$ & - & - & - & - & - & - & - & - & - & - & $\mathrm{X}$ \\
\hline Praeglobobulimina pupoides & - & - & - & - & - & - & - & - & - & - & - & - & - & - & - & $\mathrm{X}$ \\
\hline Pullenia eocenica & $\mathrm{X}$ & - & - & - & - & - & - & - & - & - & - & - & - & - & - & - \\
\hline Pullenia quinqueloba & cf. & - & - & - & - & - & - & - & - & - & - & - & - & - & - & - \\
\hline Pullenia salisburyi & $\mathrm{X}$ & - & $x$ & - & - & - & $x$ & - & $\mathrm{x}$ & - & - & - & - & - & - & - \\
\hline Quadrimorphina allomorphinoides & $\mathrm{X}$ & - & - & - & - & - & - & - & - & - & - & - & - & - & - & - \\
\hline Quinqueloculina spp. & $\mathrm{X}$ & - & - & - & - & - & - & - & - & - & - & - & - & - & - & - \\
\hline
\end{tabular}




\begin{tabular}{|c|c|c|c|c|c|c|c|c|c|c|c|c|c|c|c|c|}
\hline $\begin{array}{c}\text { Benthic foraminifers from unit Tshc } \\
\text { of Graymer and others } \\
\text { (1994a,1996), near San Pablo } \\
\text { Reservoir in Block II }\end{array}$ & $\begin{array}{l}\stackrel{2}{\circ} \\
\frac{0}{\Sigma} \\
\frac{1}{\Sigma}\end{array}$ & 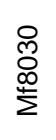 & $\begin{array}{l}\text { D } \\
\infty \\
\dddot{\infty} \\
\infty \\
\Sigma\end{array}$ & 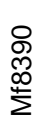 & 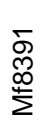 & 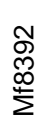 & 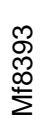 & 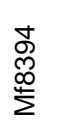 & $\begin{array}{l}\stackrel{\infty}{\infty} \\
\text { D } \\
\stackrel{\infty}{\Sigma}\end{array}$ & $\begin{array}{l}\mathscr{D} \\
\mathscr{D} \\
\infty \\
\Sigma\end{array}$ & 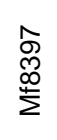 & 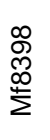 & $\begin{array}{l}\stackrel{\mathscr{D}}{ } \\
\text { 足 } \\
\sum\end{array}$ & 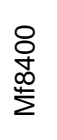 & $\begin{array}{l}\bar{o} \\
\bar{d} \\
\infty \\
\Sigma\end{array}$ & 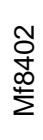 \\
\hline Sigmoilina tenuis & - & - & - & - & - & - & - & - & - & - & - & - & - & $\mathrm{x}$ & - & $\mathrm{x}$ \\
\hline Silicosigmoilina californica & $x$ & - & - & - & - & - & - & - & - & - & - & - & - & $x$ & $x$ & - \\
\hline Siphonia wilcoxensis & - & - & - & - & $x$ & - & - & $x$ & - & - & - & - & - & - & - & - \\
\hline Spiroloculina texana & $\mathrm{x}$ & - & - & - & - & - & - & - & - & - & - & - & - & - & - & - \\
\hline Spiroplectammina directa & - & - & - & - & - & - & - & - & - & - & - & - & - & - & - & $\mathrm{x}$ \\
\hline Spiroplectammina richardi & - & $\mathrm{x}$ & - & - & - & - & $\mathrm{x}$ & $\mathrm{x}$ & $\mathrm{x}$ & $\mathrm{x}$ & $\mathrm{x}$ & - & - & - & - & - \\
\hline Stilostomella gracilis & $\mathrm{x}$ & - & - & - & - & - & - & - & - & - & - & - & - & - & - & - \\
\hline Textularia plummerae & - & - & - & $\mathrm{x}$ & - & $\mathrm{x}$ & $\mathrm{x}$ & $\mathrm{x}$ & $\mathrm{x}$ & $\mathrm{x}$ & - & $x$ & $\mathrm{x}$ & $\mathrm{x}$ & $\mathrm{x}$ & $\mathrm{x}$ \\
\hline Trifarina advena californica & - & - & - & - & - & - & $\mathrm{x}$ & $\mathrm{x}$ & - & - & - & - & - & $\mathrm{x}$ & - & - \\
\hline Tritaxilina colei & $\mathrm{x}$ & - & - & $\mathrm{x}$ & $\mathrm{x}$ & - & - & - & $\mathrm{x}$ & - & - & - & - & - & - & $\mathrm{x}$ \\
\hline Trochammina globigeriniformis & - & - & - & $\mathrm{x}$ & - & - & - & - & - & - & - & $\mathrm{x}$ & $\mathrm{x}$ & - & - & $\mathrm{x}$ \\
\hline Trochamminoides conglobatulus & $\mathrm{x}$ & - & - & - & - & - & - & - & - & - & - & - & - & - & - & - \\
\hline Uvigerina lodoensis miriamae & - & - & - & - & $x$ & - & - & $x$ & - & - & - & - & - & $\mathrm{x}$ & - & - \\
\hline Vaginulinopsis asperuliformis & - & - & - & - & - & - & - & $x$ & $x$ & - & $\mathrm{x}$ & - & - & - & - & - \\
\hline Vaginulinopsis saundersi & $x$ & - & - & $x$ & $x$ & $x$ & - & - & - & - & $x$ & - & - & $x$ & - & $x$ \\
\hline Verneuilina triangulata & $\mathrm{x}$ & - & - & - & $\mathrm{x}$ & - & - & $\mathrm{x}$ & 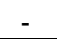 & - & & - & - & - & - & - \\
\hline
\end{tabular}

Table 8. Benthic foraminifers from unit Tshc of Graymer and others (1994a, 1996) near Briones Reservoir in Block II.

[Taxonomy of species is given in the section entitled "Taxonomic Notes." $\mathrm{X}$ indicates the species is present; cf. indicates that the specimens resemble or compare to the species]

\begin{tabular}{|c|c|c|c|c|c|}
\hline $\begin{array}{l}\text { Benthic foraminifers from unit } \\
\text { Tshc of Graymer and others } \\
\text { (1994a, 1996), near Briones } \\
\text { Reservoir in Block II }\end{array}$ & $\frac{m}{\infty}$ & $\underset{\substack{+\infty}}{\stackrel{+}{\Sigma}}$ & $\begin{array}{l}\frac{10}{\infty} \\
\infty \\
\sum \\
\sum\end{array}$ & $\begin{array}{l}0 \\
\infty \\
\infty \\
\sum \\
\sum\end{array}$ & $\begin{array}{l}\stackrel{L}{N} \\
\infty \\
\infty \\
\sum \\
\Sigma\end{array}$ \\
\hline Ammodiscus incertus & - & - & $x$ & - & - \\
\hline Amphimorphina ignota & - & - & - & - & $x$ \\
\hline Anomalinoides capitatus & - & - & - & $x$ & $x$ \\
\hline Anomalina keenae & - & - & $x$ & $x$ & $x$ \\
\hline Anomalina regina & - & - & $x$ & $x$ & - \\
\hline Aragonia aragonensis & - & - & $x$ & - & - \\
\hline Astacolus spp. & - & - & - & $x$ & $x$ \\
\hline Bathysiphon eocenicus & - & $x$ & - & - & - \\
\hline Bathysiphon santecruis & - & - & - & - & $x$ \\
\hline Bathysiphon spp. & $x$ & $x$ & $x$ & $x$ & $x$ \\
\hline Bolivina incrassata of Mallory & - & - & - & $x$ & - \\
\hline Bulimina alazaensis & - & - & - & $x$ & - \\
\hline Bulimina bradburyi & - & - & - & - & $x$ \\
\hline Bulimina macilenta & - & - & $x$ & $x$ & $x$ \\
\hline Bulimina trinitatensis & - & - & $x$ & $x$ & - \\
\hline Buliminella grata & - & - & $x$ & - & $x$ \\
\hline Buliminella robertsi & - & - & $x$ & $x$ & - \\
\hline Chilostomella oolina & - & $x$ & $x$ & - & $x$ \\
\hline Chrysalongonium elongatum & - & - & - & $x$ & $x$ \\
\hline Chrysalongonium teniucostatum & - & - & - & - & $x$ \\
\hline Cibicidoides eocaenus & $x$ & $x$ & $x$ & $x$ & $x$ \\
\hline Cibicidoides eponidiformis & - & - & - & $x$ & $x$ \\
\hline Cibicidoides praemundulus & $x$ & - & $x$ & - & - \\
\hline Cibicidoides subspiratus & - & - & $x$ & $x$ & $x$ \\
\hline Cibicidoides spp. & - & - & - & - & $x$ \\
\hline
\end{tabular}

\begin{tabular}{|c|c|c|c|c|c|}
\hline $\begin{array}{l}\text { Benthic foraminifers from unit } \\
\text { Tshc of Graymer and others } \\
\text { (1994a, 1996), near Briones } \\
\text { Reservoir in Block II }\end{array}$ & $\begin{array}{l}\frac{m}{\infty} \\
\frac{1}{2} \\
\sum\end{array}$ & $\begin{array}{l}\frac{\nabla}{\infty} \\
\frac{1}{2} \\
\frac{1}{2}\end{array}$ & $\begin{array}{l}\frac{10}{\infty} \\
\frac{1}{2} \\
\sum\end{array}$ & $\begin{array}{l}\frac{0}{\infty} \\
\infty \\
\stackrel{1}{\Sigma}\end{array}$ & 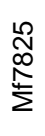 \\
\hline Clavulina anglica & - & - & - & $X$ & $x$ \\
\hline Clavulinoides californicus & - & - & $x$ & - & - \\
\hline Cyclammina simiensis & - & $\mathrm{X}$ & $\mathrm{X}$ & $X$ & $X$ \\
\hline Cyclammina spp. & - & - & $x$ & - & - \\
\hline Dentalina colei & - & - & $\mathrm{X}$ & $X$ & $\mathrm{X}$ \\
\hline Dentalina communis & - & - & $x$ & $\mathrm{X}$ & $x$ \\
\hline Dentalina jacksonensis & - & - & - & - & $X$ \\
\hline Dentalina soluta & - & $x$ & - & - & - \\
\hline Dentalina spp. & $\mathrm{X}$ & - & - & - & $X$ \\
\hline Dorothia bulletta & $\mathrm{X}$ & - & $x$ & $x$ & $x$ \\
\hline Dorothia principiensis & - & - & $X$ & $X$ & - \\
\hline Eggerella elongata & - & $x$ & - & - & $x$ \\
\hline Eponides lodoensis & $x$ & - & $x$ & $X$ & - \\
\hline Fissurina alveolata & - & - & - & cf. & - \\
\hline Fursenkoina bramletti & - & - & - & $X$ & - \\
\hline Globocassidulina globosa & - & $\mathrm{x}$ & - & - & $x$ \\
\hline Glandulina laevigata & - & - & $x$ & - & $x$ \\
\hline Glomospira charoides & - & - & - & $X$ & $x$ \\
\hline Guttulina problema & - & - & - & $X$ & $x$ \\
\hline Gyroidina orbicularis & - & - & $x$ & $X$ & - \\
\hline Gyroidina planulata & - & - & $x$ & - & $x$ \\
\hline Gyroidina soldanii & $x$ & - & $x$ & $X$ & $x$ \\
\hline Gyroidina spp. & - & - & - & - & $x$ \\
\hline Hanzawaia ammophila & - & - & - & $X$ & - \\
\hline Haplophragmoides eggeri & - & $x$ & - & - & $x$ \\
\hline
\end{tabular}




\begin{tabular}{|c|c|c|c|c|c|}
\hline $\begin{array}{c}\text { Benthic foraminifers from unit } \\
\text { Tshc of Graymer and others } \\
\text { (1994a, 1996), near Briones } \\
\text { Reservoir in Block II }\end{array}$ & $\begin{array}{l}\frac{m}{\infty} \\
\substack{\infty \\
p^{2}}\end{array}$ & $\begin{array}{l}\frac{J}{\infty} \\
\substack{\infty \\
\perp}\end{array}$ & $\begin{array}{l}\frac{10}{\infty} \\
\substack{\infty \\
\sum}\end{array}$ & $\begin{array}{l}0 \\
\frac{0}{\infty} \\
\frac{1}{1} \\
\sum\end{array}$ & $\begin{array}{l}\stackrel{1}{N} \\
\infty \\
\stackrel{\infty}{\Sigma} \\
\sum\end{array}$ \\
\hline Haplophragmoides spp. & - & $x$ & $x$ & $x$ & - \\
\hline Hormosina spp. & - & - & - & - & $x$ \\
\hline Karreriella chapapotensis & - & - & - & $x$ & $x$ \\
\hline Karreriella elongata & - & - & $x$ & $x$ & $x$ \\
\hline Lagena costata & - & - & $x$ & - & - \\
\hline Lagena hexagona & - & - & cf. & - & - \\
\hline Lagena vulgaris & - & - & - & $x$ & - \\
\hline Lenticulina arcuatostriata & - & - & - & $x$ & - \\
\hline Lenticulina cultrata & - & - & - & $x$ & - \\
\hline Lenticulina limbosa hockleyensis & - & - & $x$ & $x$ & $x$ \\
\hline Lenticulina pseudocultratus & - & - & $x$ & - & - \\
\hline Lenticulina pseudovortex & - & - & - & $x$ & $x$ \\
\hline Lenticulina spp. & $x$ & $x$ & $x$ & $x$ & $x$ \\
\hline Lenticulina terryi & - & - & $x$ & $x$ & $\mathrm{X}$ \\
\hline Lenticulina vortex & - & - & $x$ & - & $\mathrm{X}$ \\
\hline Lituotuba lituiformis & - & - & $x$ & - & - \\
\hline Loxostomoides applinae & - & - & - & $x$ & - \\
\hline Marginulina exima & - & - & $x$ & - & - \\
\hline Marginulina subbullata & - & - & $x$ & $x$ & - \\
\hline Nonionella spp. & - & - & - & $x$ & - \\
\hline Nodosaria deliciae & $x$ & $x$ & $x$ & $x$ & - \\
\hline Nodosaria latejugata & $x$ & $x$ & $x$ & - & - \\
\hline Nodosaria longiscata & $x$ & - & $x$ & $x$ & - \\
\hline Nodosaria spp. & $x$ & $x$ & $x$ & $x$ & - \\
\hline Nodosarella advena & - & - & $x$ & $x$ & - \\
\hline Nodosarella atlantisae hispidula & - & - & - & $x$ & - \\
\hline Nuttaloides truempyi & $x$ & - & $x$ & $x$ & $x$ \\
\hline Oridorsalis umbonatus & $x$ & $x$ & $x$ & $x$ & $x$ \\
\hline
\end{tabular}

\begin{tabular}{|c|c|c|c|c|c|}
\hline $\begin{array}{c}\text { Benthic foraminifers from unit } \\
\text { Tshc of Graymer and others } \\
\text { (1994a, 1996), near Briones } \\
\text { Reservoir in Block II }\end{array}$ & $\begin{array}{l}\frac{m}{\infty} \\
\frac{1}{2} \\
\sum\end{array}$ & 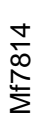 & $\begin{array}{l}\frac{1}{\infty} \\
\frac{1}{2} \\
\sum^{+1}\end{array}$ & $\begin{array}{l}\frac{0}{\infty} \\
\stackrel{\infty}{\infty} \\
\sum\end{array}$ & 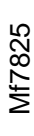 \\
\hline Orthomorphina spp. & - & - & $x$ & - & - \\
\hline Osangularia mexicana & - & $x$ & $x$ & $x$ & $x$ \\
\hline Plectina garzaensis & $\mathrm{X}$ & - & $x$ & $x$ & - \\
\hline Plectofrondicularia paucicostata & - & - & $x$ & $x$ & - \\
\hline Plectofrondicularia vaughani & - & - & - & $x$ & - \\
\hline Pleurostomella acuta & - & - & $x$ & - & - \\
\hline Pleurostomella nuttalli & - & - & $x$ & $x$ & - \\
\hline Pseudonodosaria inflata & - & $x$ & - & - & - \\
\hline Pullenia quinqueloba & - & - & $x$ & - & - \\
\hline Pullenia salisburyi & - & - & $x$ & $x$ & - \\
\hline Reophax subfusiformis & cf. & - & - & - & - \\
\hline Saccammina spp. & $x$ & - & - & - & - \\
\hline Silicosigmoilina californica & $x$ & $x$ & - & $x$ & $x$ \\
\hline Spiroloculina texana & - & - & - & $x$ & - \\
\hline Spiroplectammina directa & - & $x$ & - & - & $x$ \\
\hline Spiroplectammina richardi & $x$ & - & $x$ & $x$ & $x$ \\
\hline Stilostomella adolphina & - & - & $x$ & $x$ & - \\
\hline Stilostomella lepidula & - & - & $x$ & - & $\mathrm{x}$ \\
\hline Textularia adalta & $x$ & - & $x$ & - & $x$ \\
\hline Trifarina advena californica & - & - & $x$ & - & - \\
\hline Tritaxilina colei & $x$ & $x$ & $x$ & $x$ & - \\
\hline Trochammina globigeriniformis & $x$ & $x$ & $x$ & $x$ & $x$ \\
\hline Trochammina spp. & - & - & - & - & $x$ \\
\hline Trochamminoides conglobatulus & - & - & $x$ & - & - \\
\hline Vaginulinopsis asperuliformis & - & - & $x$ & $x$ & - \\
\hline Vaginulinopsis saundersi & $x$ & - & - & - & - \\
\hline Verneuilina triangulata & $x$ & $x$ & $x$ & $x$ & - \\
\hline
\end{tabular}

One sample, Mf7971, originally believed to be from the La Juntas Shale(?), and two additional samples, Mf7967 and Mf7968, originally believed to be from the Briones Sandstone (E.E. Brabb, written commun., 1994), contain microfossils that are early Miocene in age (table 9) and are here interpreted to represent the San Ramon Sandstone. This fauna is assigned to the early Miocene Saucesian Stage based on the co-occurrence of Buccella mansfieldi oregonensis, Nonionella miocenica, and Virgulinella pertusa, which first appear in the Oligocene, and Bolivina advena striatella, Fursenkoina californiensis, and Nonionella costifera, which first appear in the early Miocene. Deposition occurred at outer neritic depths (50 to $150 \mathrm{~m}$ ) based on the presence of numerous benthic foraminiferal species that have upper depth limits in the outer neritic biofacies and near the shelf edge, such as Bolivina advena striatella and Fursenkoina. Virgulinella pertusa ranges from the Oligocene through the Miocene primarily in the Arctic and Northern Europe (McDougall, 1995). Its presence so far south in western North America indicates cooler climatic conditions. 
Table 9. Benthic foraminifers from the San Ramon Sandstone (?) in Block II.

[Taxonomy of species is given in the section entitled "Taxonomic Notes." X indicates that the species is present; cf. indicates that the specimens resemble or compare to the species]

\begin{tabular}{|c|c|c|c|}
\hline $\begin{array}{l}\text { Benthic foraminifers from the } \\
\text { San Ramon Sandstone (?) in } \\
\text { Block II }\end{array}$ & $\begin{array}{l}\hat{\mathscr{Q}} \\
\stackrel{1}{\Sigma} \\
\sum\end{array}$ & 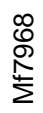 & $\begin{array}{l}\frac{1}{S} \\
\frac{N}{\Sigma} \\
\sum_{\Sigma}^{+}\end{array}$ \\
\hline Bolivina advena striatella & $x$ & - & - \\
\hline Buccella mansfieldi oregonensis & $x$ & $x$ & $x$ \\
\hline Fursenkoina californiensis & - & $x$ & - \\
\hline Fursenkoina californiensis & cf. & - & cf. \\
\hline Nonionella costifera & $x$ & - & $x$ \\
\hline Nonionella miocenica & - & $x$ & - \\
\hline Nonionella schencki & - & $x$ & - \\
\hline Virgulinella pertusa & - & $\mathrm{X}$ & - \\
\hline
\end{tabular}

Deposition of unit Tshc occurred at lower middle bathyal to lower bathyal (1,500-4,000 m). The depth interpretation is based on the upper depth limits of Alabamina wilcoxensis, Anomalinoides capitatus, Cibicidoides grimsdalei, Gyroidina soldanii, and Nuttaloides truempyi, which occur in the lower middle bathyal or lower bathyal biofacies. Considerable material has been transported from the outer shelf and upper slope. The source of the transported material is the primary difference between the two areas sampled. The area near Briones Reservoir includes more material transported from the upper slope whereas the area north of the San Pablo Reservoir includes more material transported from the outer shelf.

The San Ramon Sandstone unconformably overlies unit Tshc near the San Pablo and Briones Reservoirs. Microfossils from the San Ramon Sandstone, unnamed tuffaceous sandstone (unit Tts of Graymer and others, 1994a), and younger formations collected near the San Pablo and Briones Reservoirs were examined by Kleinpell (1938, table XIII, p. 62-65) and assigned to the Oligocene through Miocene. The stratigraphically oldest samples examined by Kleinpell (1938) were from the San Ramon Formation and Kirker Tuff. Although these meager foraminiferal assemblages are assigned to the Oligocene Zemorrian Stage, the presence of Sphaeroidina bulloides and Buccella mansfieldi oregonensis (Epistomina ramonensis of Kleinpell, 1938) indicate that this assemblage is early Miocene in age and should be assigned to the Saucesian Stage. This interpretation is consistent with the molluscan interpretation that considers the San Ramon Sandstone to be early Miocene in age (Weaver and others, 1944; Addicott, 1970; and Graymer and others, 2002a).

The Sobrante Sandstone overlies the San Ramon Sandstone and an unnamed tuffaceous sandstone (unit Tts) of Graymer and others (1994a). Two samples collected from the Sobrante Sandstone contain benthic foraminiferal assemblages that suggest an early Miocene (Saucesian) age (table 10). This interpretation is based on the first appearance of Bolivina advena, B. floridana, Siphogenerina branneri, and S. transversa, and the last appearance of Gaudryina trintatensis, Haplophragmoides trullisata, and Martinotiella communis. The latter species occurs only in Mf1093. Sample Mf1094 does not contain any species that restrict the upper age limit of this sample. Samples examined by Kleinpell (1938, table XIII, p. 62-65) from the "Concord Formation" (Sobrante Sandstone of Graymer and others, 1994a) near Briones Reservoir indicate the early Miocene Saucesian Stage. Deposition of the Sobrante Sandstone occurred at upper bathyal depths (150-500 m). 
Table 10. Benthic foraminifers from the Neogene Sobrante Sandstone, unit Tus of Graymer and others (1994a, 1996), Claremont Shale, and Oursan Sandstone near San Pablo and Briones Reservoirs in Block II.

[Taxonomy of species is given in the section entitled "Taxonomic Notes." X indicates the species is present; cf. indicates thatthe specimens resemble or compare to the species. Samples that are barren of microfossils or contain few to rare nondiagnostic species include: Mf906, Mf911, Mf7817, Mf7818, Mf7819, Mf7821, Mf7852, Mf7857, Mf7858, Mf8023, Mf8024, Mf8025, Mf8026, and Mf8114. Samples from the Briones Sandstone that are barren of microfossils or contain few to rare non diagnostic species include: Mf7854, Mf7855, Mf7856, and Mf7877. No faunal data available for samples Mf1736, Mf1740, Mf1741, and Mf1742]

\begin{tabular}{|c|c|c|c|c|c|c|c|c|c|c|c|c|c|c|c|c|}
\hline \multirow{2}{*}{$\begin{array}{l}\text { Benthic foraminifers from the } \\
\text { Neogene Sobrante Sandstone, unit } \\
\text { Tus of Graymer and others (1994a, } \\
\text { 1996), Claremont Shale, and } \\
\text { Oursan Sandstone near San Pablo } \\
\text { and Briones Reservoirs in Block II }\end{array}$} & \multicolumn{5}{|c|}{ Sobrante Sandstone } & \multirow{2}{*}{ 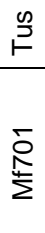 } & \multicolumn{5}{|c|}{ Claremont Shale } & \multicolumn{5}{|c|}{ Oursan Sandstone } \\
\hline & 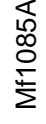 & $\begin{array}{l}\text { Љ } \\
\text { O } \\
\frac{1}{\Sigma}\end{array}$ & 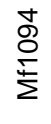 & $\begin{array}{l}\hat{\infty} \\
\infty \\
\sum \\
\sum\end{array}$ & $\begin{array}{l}\frac{10}{\sigma} \\
\frac{1}{\Sigma}\end{array}$ & & $\begin{array}{l}\frac{N}{O} \\
\frac{+}{\Sigma}\end{array}$ & $\begin{array}{l}\frac{m}{\sigma} \\
\frac{P}{2}\end{array}$ & $\begin{array}{l}\frac{+}{O} \\
\frac{+}{\Sigma}\end{array}$ & $\begin{array}{l}\frac{0}{\sigma} \\
\frac{1}{2}\end{array}$ & $\begin{array}{l}\hat{\sigma} \\
\frac{0}{\Sigma} \\
\frac{1}{\Sigma}\end{array}$ & $\begin{array}{l}\frac{\sigma}{\sigma} \\
\frac{\sigma}{\Sigma}\end{array}$ & 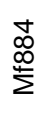 & $\begin{array}{l}\infty \\
\infty \\
\infty \\
\stackrel{\infty}{\Sigma}\end{array}$ & $\begin{array}{l}\text { ㅇ } \\
\text { O্+ } \\
\sum\end{array}$ & $\begin{array}{l}\stackrel{1}{\circ} \\
\text { O } \\
\sum^{+}\end{array}$ \\
\hline Baggina spp. & - & - & - & - & - & - & - & - & - & - & - & - & - & - & - & $\mathrm{X}$ \\
\hline Bolivina advena striatella & - & - & - & $\mathrm{X}$ & $x$ & - & - & - & - & - & - & $x$ & - & - & $x$ & - \\
\hline Bolivina advena & - & - & $\mathrm{X}$ & $x$ & - & - & - & $x$ & - & $\mathrm{X}$ & $\mathrm{X}$ & - & - & - & - & $X$ \\
\hline Bolivina floridana & - & - & cf. & - & - & - & - & - & - & - & - & - & - & - & - & - \\
\hline Bolivina guadeloupae & - & - & - & - & - & - & - & - & - & - & - & - & - & - & - & $\mathrm{X}$ \\
\hline Bolivina marginata & - & $x$ & $x$ & $x$ & - & $x$ & - & - & - & - & - & - & - & - & - & - \\
\hline Bolivina pseudospissa & - & - & - & - & - & - & - & - & - & - & cf. & - & - & - & - & - \\
\hline Bolivina salinasensis & - & - & - & $x$ & - & - & - & - & - & - & - & - & - & - & - & - \\
\hline Bolivina tumida & - & - & - & - & $x$ & - & $x$ & - & $x$ & - & - & $x$ & $x$ & $x$ & $x$ & - \\
\hline Buliminella curta & - & - & $x$ & - & - & $x$ & - & - & - & - & - & - & - & - & - & - \\
\hline Buliminella subfusiformis & - & - & - & $x$ & - & - & $x$ & - & $x$ & $x$ & $\mathrm{X}$ & $x$ & - & $\mathrm{X}$ & $x$ & $x$ \\
\hline Cibicidoides floridanus & - & $x$ & - & - & - & - & - & - & - & - & - & - & - & - & - & - \\
\hline Cribrostomoides cretacea & $x$ & - & - & - & - & - & - & - & - & - & - & - & - & - & - & - \\
\hline Frondicularia spp. & - & - & - & - & - & - & - & - & $x$ & - & - & - & - & - & - & - \\
\hline Fursenkoina californiensis & - & - & - & $\mathrm{X}$ & - & - & - & $x$ & $x$ & $x$ & $\mathrm{X}$ & - & $x$ & - & - & - \\
\hline Gaudryina trinitatensis & - & cf. & - & - & - & - & - & - & - & - & - & - & - & - & - & - \\
\hline Gyroidina soldanii & - & $x$ & - & - & - & - & - & - & - & - & - & - & - & - & - & - \\
\hline Haplophragmoides trullisata & - & cf. & - & - & - & - & - & - & - & - & - & - & - & - & - & - \\
\hline Marginulina beali & - & - & - & - & - & $x$ & - & - & - & - & - & - & - & - & - & - \\
\hline Martinotiella communis & - & cf. & - & - & - & - & - & - & - & - & - & - & - & - & - & - \\
\hline Nonionella costifera & - & - & $x$ & $x$ & $x$ & - & - & - & - & - & $\mathrm{X}$ & $x$ & $x$ & $x$ & $\mathrm{X}$ & - \\
\hline Nonionella miocenica & - & - & - & $x$ & - & - & - & $x$ & - & $x$ & - & $x$ & - & - & - & - \\
\hline Nonionella spp. & - & - & - & - & - & $x$ & - & - & - & - & - & - & - & - & $x$ & - \\
\hline Nonion spp. & - & - & - & - & - & - & - & - & $x$ & $\mathrm{X}$ & - & - & - & - & - & - \\
\hline Plectofrondicularia miocenica & - & $x$ & - & - & - & - & - & - & - & - & - & - & - & - & - & - \\
\hline Silicosigmoilina californica & $x$ & - & - & - & - & - & - & - & - & - & - & - & - & - & - & - \\
\hline Siphogenerina branneri & - & - & cf. & - & - & - & - & - & - & - & - & - & - & - & - & - \\
\hline Siphogenerina collomi & - & - & - & $x$ & - & - & - & - & - & - & - & - & - & - & - & - \\
\hline Siphogenerina transversa & - & cf. & - & - & - & - & - & - & - & - & - & - & - & - & - & - \\
\hline Siphogenerina spp. & - & $x$ & $\mathrm{x}$ & - & - & $x$ & - & - & - & - & - & - & - & - & - & - \\
\hline Uvigerinella californica & - & - & - & - & - & - & - & - & $x$ & - & - & - & - & - & - & - \\
\hline Uvigerinella obesa & - & - & - & - & - & - & - & - & - & $x$ & - & - & - & - & $\mathrm{X}$ & - \\
\hline Valvulineria californica s.s. & - & - & - & $\mathrm{X}$ & - & $\mathrm{x}$ & - & - & - & - & $\mathrm{X}$ & $\mathrm{X}$ & $\mathrm{X}$ & $X$ & $X$ & - \\
\hline
\end{tabular}


Two additional samples, Mf887 and Mf915, were collected in a unit identified as the Sobrante Sandstone, but the assemblages in these samples suggest a middle Miocene age based on the presence of Bolivina advena striatella, Valvulineria californica, and Siphogenerina collomi and should be assigned to the Relizian or Luisian Stage. Similarly, sample Mf701, which occurs in unnamed Miocene to Pliocene sedimentary and volcanic rocks (unit Tus of Graymer and others, 1994a, 1996), contains a foraminiferal assemblage that includes Marginulina beali, Siphogenerina spp., and Valvulineria californica. The age of this assemblage is middle Miocene (probably Luisian Stage) and therefore suggests that it probably represents a member of the Monterey Group.

Seven samples were examined from the Claremont Shale, which overlies the Sobrante Sandstone; two samples were barren or contained only poorly preserved fragments (Mf906 and Mf911). The most common fauna is a low diversity, poorly preserved assemblage consisting of foraminiferal fragments or specimens of Bolivina or Buliminella (Mf912; table 10). This fauna is Miocene age and represents deposition at bathyal depths $(>150 \mathrm{~m})$. The fauna in the remaining five samples is a more diverse, better preserved assemblage that contains specimens of Bolivina advena, Fursenkoina californiensis, Nonionella (N. costifera and N. miocenica), Uvigerinella (U. californica and U. obesa), and Valvulineria californica (Mf913 through Mf917; table 10). The overlapping ranges of these species indicate the samples are middle Miocene in age and assigned to the Luisian Stage. Deposition of this fauna occurred at upper bathyal to upper middle bathyal depths $(500-1,500 \mathrm{~m})$.

Five samples from the Oursan Sandstone contain benthic foraminifers (table 10). These assemblages are middle Miocene in age and assigned to the Luisian Stage based on Bolivina advena striatella, Bolivina tumida, Buliminella subfusiformis, Nonionella costifera, and Valvulineria californica. Deposition occurred at upper bathyal depths (150-500 m).

Microfossiliferous samples from the Tice Shale (3 samples), Hambre Sandstone (7), and Rodeo Shale (4) were examined (table 11). All three formations contain similar foraminiferal assemblages that are middle to late Miocene in age and assigned to the Mohnian Stage on the basis of the presence of Fursenkoina californiensis grandis, $F$. californiensis ticensis, Nonionella montereyana, $N$. multicamerata, Uvigerina subperegrina, and Valvulineria araucana. A single sample, Mf878, located in a small outcrop identified as unit Tms in the midst of an area mapped as Hambre Sandstone, contains a single species, Fursenkoina californiensis ticensis. This assemblage is most similar to the Hambre Sandstone or Rodeo Shale, and is therefore considered with these middle to upper Miocene units. Deposition of the Tice Shale occurred at upper bathyal depths (150-500 m). Deposition of the Hambre Sandstone and Rodeo Shale occurred at outer neritic depths $(50-150 \mathrm{~m})$. The difference in deposition is based on the noticeable decline in species with upper depth limits in the upper bathyal biofacies in the Hambre Sandstone and Rodeo Shale, and on the dominance of neritic species. 
Table 11. Benthic foraminifers from the Neogene Tice Shale, Hambre Sandstone and Rodeo Shale near San Pablo and Briones Reservoirs in Block II.

[Taxonomy of species is given in the section entitled "Taxonomic Notes." X indicates the species is present; cf. indicates thatthe specimens resemble or compare to the species. Samples that are barren of microfossils or contain few to rare nondiagnostic species include: Mf1100, Mf7821, MF7852, Mf7857, Mf7858, Mf8129, Mf8130, Mf8131, Mf8139, Mf8140, and Mf8143. No faunal data available for samples Mf1533, Mf1734, Mf1735, Mf1737, Mf1742, Mf1743, Mf1744]

\begin{tabular}{|c|c|c|c|c|c|c|c|c|c|c|c|c|c|c|}
\hline \multirow{2}{*}{$\begin{array}{c}\text { Benthic foraminifers from the } \\
\text { Neogene Tice Shale, Hambre } \\
\text { Sandstone and Rodeo Shale near } \\
\text { San Pablo and Briones Reservoirs } \\
\text { in Block II }\end{array}$} & \multicolumn{3}{|c|}{ Tice Shale } & \multicolumn{7}{|c|}{ Hambre Sandstone } & \multicolumn{4}{|c|}{ Rodeo Shale } \\
\hline & \begin{tabular}{l} 
N \\
$\frac{\infty}{\Sigma}$ \\
\multirow{2}{*}{}
\end{tabular} & 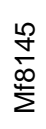 & $\begin{array}{l}\stackrel{\varphi}{2} \\
\infty \\
\dot{\infty}\end{array}$ & $\begin{array}{l}\text { D } \\
\infty \\
\sum\end{array}$ & $\begin{array}{l}\hat{O} \\
\text { O } \\
\sum\end{array}$ & 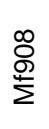 & $\frac{\infty}{\frac{\infty}{\sigma}}$ & $\begin{array}{l}\mathbb{N} \\
\mathbb{N} \\
\stackrel{N}{\Sigma} \\
\stackrel{2}{\Sigma}\end{array}$ & 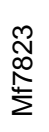 & 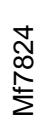 & 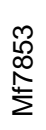 & $\begin{array}{l}\infty \\
\infty \\
\infty \\
\Sigma\end{array}$ & $\begin{array}{l}\bar{f} \\
\dot{\infty} \\
\dot{\infty}\end{array}$ & $\frac{\stackrel{N}{+}}{\stackrel{\infty}{\infty}}$ \\
\hline Bolivina advena & - & - & - & - & cf. & - & - & - & - & - & - & - & - & - \\
\hline Bolivina guadeloupae & - & - & - & - & $x$ & $x$ & - & - & - & - & - & - & - & - \\
\hline Bolivina spp. & - & - & - & - & - & - & $x$ & - & - & - & - & - & - & - \\
\hline Buliminella elegantissima & $x$ & - & $x$ & - & - & - & - & $\mathrm{X}$ & - & - & - & - & - & - \\
\hline Buliminella subfusiformis & - & - & - & - & $x$ & - & - & - & - & - & - & - & - & - \\
\hline Fursenkoina bramletti & - & - & - & - & - & - & - & - & $x$ & - & - & - & - & - \\
\hline Fursenkoina californiensis & - & $x$ & - & - & $x$ & $x$ & $x$ & $\mathrm{X}$ & - & $x$ & $x$ & - & $x$ & $x$ \\
\hline Fursenkoina californiensis grandis & - & - & - & - & - & - & - & - & - & - & - & $\mathrm{X}$ & - & - \\
\hline Fursenkoina californiensis ticensis & $x$ & - & $\mathrm{x}$ & $x$ & $x$ & $x$ & - & - & - & - & - & $x$ & - & - \\
\hline Globobulimina spp. & - & - & - & - & - & - & - & - & $x$ & - & - & - & - & - \\
\hline Gyroidina spp. & - & - & - & - & - & - & - & $\mathrm{X}$ & - & - & - & - & - & - \\
\hline Haplophragmoides spp. & - & - & - & - & - & - & - & - & - & - & $\mathrm{X}$ & - & - & - \\
\hline Nonionella costifera & - & $x$ & - & - & $x$ & - & - & - & - & - & $x$ & - & - & - \\
\hline Nonionella miocenica & $x$ & - & $x$ & - & $x$ & - & - & - & - & - & - & $x$ & - & - \\
\hline Nonionella multicamerata & - & - & - & - & - & - & - & $\mathrm{X}$ & $x$ & $x$ & - & - & - & - \\
\hline Nonionella montereyana & - & $x$ & - & - & - & - & - & - & - & - & - & - & $x$ & $x$ \\
\hline Praeglobobulimina ovula & - & - & - & - & - & $x$ & - & - & - & - & - & - & - & - \\
\hline Quinqueloculina spp. & - & - & - & - & - & - & - & - & $x$ & - & - & - & - & - \\
\hline Uvigerina subperegrina & - & - & - & - & - & $x$ & - & - & - & - & - & - & - & - \\
\hline Valvulineria araucana & cf. & - & cf. & - & - & - & - & - & - & - & - & - & - & - \\
\hline
\end{tabular}

Of the overlying units, only the Briones Sandstone, Neroly Sandstone, and the unnamed sedimentary and volcanic rock unit (Tus) were sampled for microfossils (table 12). Samples from the Briones and Neroly Sandstones were barren of microfossils or contained an assemblage with few to no diagnostic species. Although not well preserved or common, microfossils were found in unit Tus. Diagnostic species present include Fursenkoina californiensis, F. californiensis ticensis, N. montereyana, and rare Concavella gyroidinaformis, which indicate a late middle to late Miocene age and are assigned to the early Mohnian Stage. The fauna also indicates that deposition occurred at slightly shallower depths, probably near the shelf edge (approximately $150 \mathrm{~m}$ ).

Vertebrate and invertebrate remains indicate that these younger formations range in age from late middle Miocene to early Pliocene in age (Stirton, 1939; Wagner, 1978). This age range is also supported by K/Ar dates from the Briones Sandstone (14.5 $\pm 0.4 \mathrm{Ma})$, Lafayette Tuff of Graymer and

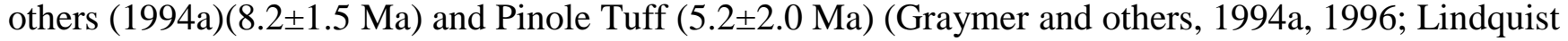
and Morganthaler, 1991; Sarna-Wojcicki, 1976). 
Table 12. Benthic foraminifers from the Neogene units Tus and Tms near San Pablo and Briones Reservoirs in Block II.

[Taxonomy of species is given in the section entitled "Taxonomic Notes." X indicates the species is present; cf. indicates thatthe specimens resemble or compare to the species. Samples that are barren of microfossils or contain few to rare nondiagnostic species include: Mf8023, Mf8024, Mf8025, Mf8026, and Mf8144. Mf8129 from the Neroly Sandstone is barren. Samples Mf3265, Mf3266, and Mf3267 from the middle of the Block II, do not contain diagnostic microfossils or are barren of microfossils]

\begin{tabular}{|c|c|c|c|c|c|c|c|c|c|c|c|}
\hline \multirow{2}{*}{$\begin{array}{l}\text { Benthic foraminifers from the } \\
\text { Neogene units Tus and Tms near } \\
\text { the San Pablo and Briones } \\
\text { Reservoirs in Block II }\end{array}$} & \multicolumn{10}{|c|}{ Unnamed sedimentary and volcanic rocks (Tus) } & \multirow{2}{*}{ 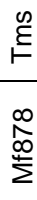 } \\
\hline & $\begin{array}{l}\stackrel{2}{N} \\
\infty \\
\sum \\
\Sigma\end{array}$ & $\begin{array}{l}\hat{N} \\
\infty \\
\sum\end{array}$ & $\begin{array}{l}\infty \\
\infty \\
\infty \\
\sum\end{array}$ & $\begin{array}{l}\frac{0}{5} \\
\frac{\infty}{2} \\
\sum\end{array}$ & 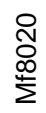 & $\begin{array}{l}\bar{N} \\
\stackrel{\infty}{\alpha} \\
\sum_{\Sigma}^{\infty}\end{array}$ & $\begin{array}{l}\text { Nิ } \\
\stackrel{0}{2} \\
\sum_{\Sigma}^{2}\end{array}$ & $\begin{array}{l}\hat{N} \\
\text { Oे } \\
\stackrel{\infty}{\Sigma}\end{array}$ & $\begin{array}{l}\stackrel{\infty}{\sim} \\
\underset{\infty}{\infty} \\
\stackrel{\infty}{\Sigma}\end{array}$ & 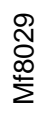 & \\
\hline Bolivina spp. & - & - & - & $\mathrm{x}$ & - & - & $\mathrm{X}$ & - & - & - & - \\
\hline Buliminella elegantissima & $x$ & - & - & - & - & - & - & - & - & - & - \\
\hline Concavella gyroidinaformis & - & - & - & - & - & - & - & - & $\mathrm{X}$ & - & - \\
\hline Cyclammina spp. & - & - & - & - & - & - & - & $x$ & - & - & - \\
\hline Fursenkoina californiensis & $x$ & - & $x$ & - & $x$ & $x$ & - & $x$ & $x$ & $x$ & - \\
\hline Fursenkoina californiensis ticensis & $x$ & $x$ & - & - & - & - & - & - & - & - & $x$ \\
\hline Gyroidina spp. & - & - & $x$ & - & - & - & - & - & - & - & - \\
\hline Haplophragmoides spp. & - & - & - & - & - & - & - & $x$ & - & - & - \\
\hline Nonionella miocenica & $x$ & - & - & - & - & - & - & - & - & - & - \\
\hline Nonionella montereyana & - & - & - & - & - & - & - & $x$ & $x$ & - & - \\
\hline Nonionella spp. & - & - & $x$ & $x$ & $x$ & $x$ & $x$ & - & - & $x$ & - \\
\hline Trifarina angulosa & $x$ & - & - & - & - & - & - & - & - & - & - \\
\hline Valvulineria araucana & cf. & - & - & - & - & - & - & - & - & - & - \\
\hline
\end{tabular}

\section{Dublin Area}

The stratigraphic sequence in the Dublin area is similar to the sequence near the Briones and San Pablo Reservoirs. No Paleogene samples were taken in this area, where the Sobrante Sandstone rests unconformably on the Cretaceous Great Valley sequence and is in turn overlain by the Claremont Shale, Rodeo Shale, Hambre Sandstone, Tice Shale, Oursan Sandstone, Briones Sandstone, and an unnamed sedimentary and volcanic rock unit (Tus). Microfossil assemblages are rare in this area. Many samples were barren or contained only unidentifiable fragments (tables 13 and 14).

The only fossiliferous sample from the Sobrante Sandstone, Mf3334, contains a middle Miocene assemblage that includes Siphogenerina branneri and Valvulineria californica. The presence of Pullenia miocenica suggests deposition in the upper middle bathyal biofacies (500-1,500 m).

There are three fossiliferous samples from the Claremont Shale, one from the Oursan Sandstone, and five from the undifferentiated Rodeo Shale, Hambre Sandstone, Tice Shale, and Oursan Sandstone (table 11). Foraminifers from these formations are long-ranging and suggest only a Miocene or younger age. Sample Mf 7896 from the undifferentiated Rodeo Shale, Hambre Sandstone, Tice Shale, and Oursan Sandstone contains Marginulina beali, which indicates a middle Miocene age. Deposition occurred at upper bathyal depths or greater $(\geq 150 \mathrm{~m})$.

The only fossiliferous sample from the Sobrante Sandstone, Mf3334, contains a middle Miocene assemblage which includes Siphogenerina branneri and Valvulineria californica. The presence of Pullenia miocenica suggests deposition in the upper middle bathyal biofacies (500-1,500 m).

Foraminifers from the Claremont Shale, Oursan Sandstone, and the undifferentiated Rodeo Shale, Hambre Sandstone, Tice Shale, and Oursan Sandstone (table 11) are long-ranging and suggest only a Miocene or younger age. Sample Mf 7896 from the undifferentiated Rodeo Shale, Hambre 
Sandstone, Tice Shale, and Oursan Sandstone contains Marginulina beali, which indicates a middle Miocene age. Deposition occurred at upper bathyal depths or greater $(\geq 150 \mathrm{~m})$.

One fossiliferous sample was examined from the Briones Sandstone, and it contains only one long-ranging Miocene and younger species that does not limit the age (table 13). Deposition occurred at upper bathyal depths $(150-500 \mathrm{~m})$.

Two samples, Mf3272 and Mf3337, are located in an area mapped as an unnamed sedimentary rock unit of Cretaceous age (Ku) (Graymer and others, 1996). Both samples contain a foraminiferal assemblage that is late Miocene or younger based on the presence of Bolivina argentea, Cassidulina transluscens, Cibicides fletcheri, C. mckannai, Epistominella bradyana, E. smithi, and Oridorsalis tenera (table 14). Deposition occurred at upper middle bathyal depths (500-1,500 m) with transport from the shelf. This sample occurs near other samples (Mf3335, Mf7800, and Mf7801) that are also in the area mapped as unit $\mathrm{Ku}$ and near the Block II/VII bounding fault, so it is probably from a previously unidentified fault sliver of a younger unit.

Table 13. Benthic foraminifers from Neogene units (Graymer and others, 1994a, 1996) near Dublin in Block II. [Taxonomy of species is given in the section entitled "Taxonomic Notes.". X indicates the species is present. Samples that are barren or do not contain diagnostic microfossils include: Mf3332, Mf3333, Mf3336, Mf3338, Mf3346, Mf3347, Mf3348, Mf3349, Mf3350, Mf3353, Mf7891, Mf7892, Mf7893, Mf7894, Mf7895, Mf7898, Mf7900, Mf7901, Mf7903, Mf8048, Mf8049, Mf8052, Mf8053, Mf8403, Mf8404, Mf8405, Mf8406, and Mf8407]

\begin{tabular}{|c|c|c|c|c|c|c|c|c|c|c|c|c|}
\hline \multirow{2}{*}{$\begin{array}{l}\text { Benthic foraminifers from } \\
\text { Neogene units near Dublin in } \\
\text { Block II }\end{array}$} & \multirow{2}{*}{ 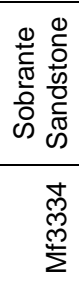 } & \multicolumn{3}{|c|}{ Claremont Shale } & \multirow{2}{*}{ 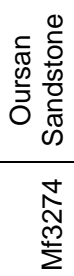 } & \multicolumn{5}{|c|}{$\begin{array}{l}\text { Rodeo Shale, Hambre Sandstone, } \\
\text { Tice Shale, and Oursan Sandstone, } \\
\text { undivided }\end{array}$} & \multirow{2}{*}{ 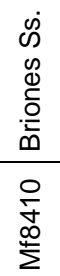 } & \multirow{2}{*}{ 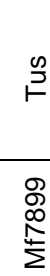 } \\
\hline & & 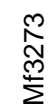 & 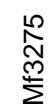 & $\begin{array}{c}\infty \\
\stackrel{N}{N} \\
\sum\end{array}$ & & $\begin{array}{l}\mathscr{\wp} \\
\stackrel{\infty}{\infty} \\
\sum^{\perp}\end{array}$ & 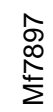 & $\begin{array}{l}\text { N } \\
\text { ᄋ } \\
\stackrel{N}{\Sigma} \\
\sum\end{array}$ & $\begin{array}{l}\infty \\
\stackrel{o}{+} \\
\infty \\
\sum\end{array}$ & 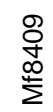 & & \\
\hline Bathysiphon spp. & - & - & - & - & - & - & $\mathrm{X}$ & - & - & - & - & - \\
\hline Bolivina advena & 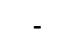 & - & $\mathrm{x}$ & - & - & - & - & - & - & - & - & - \\
\hline Bolivina advena striatella & $x$ & . & - & - & - & $\mathrm{x}$ & - & - & - & - & - & - \\
\hline Bolivina spp. & - & $\mathrm{x}$ & $\mathrm{X}$ & - & $x$ & $\mathrm{x}$ & - & - & - & - & - & $x$ \\
\hline Buliminella subfusiformis & $x$ & - & $\mathrm{x}$ & - & - & $\mathrm{x}$ & - & - & - & - & - & - \\
\hline Buliminella spp. & - & $x$ & - & - & - & - & - & - & - & - & - & - \\
\hline Cassidulina panzana & - & - & - & - & - & $\mathrm{x}$ & - & - & - & - & - & - \\
\hline Epistominella subperuviana & $\mathrm{x}$ & - & $\mathrm{x}$ & - & - & $\mathrm{x}$ & - & - & - & - & - & - \\
\hline Fursenkoina californiensis & - & - & - & $\mathrm{X}$ & - & $\mathrm{x}$ & $\mathrm{x}$ & $x$ & $\mathrm{x}$ & $\mathrm{x}$ & $\mathrm{x}$ & - \\
\hline Fursenkoina californiensis grandis & - & - & - & - & - & - & - & $\mathrm{x}$ & - & - & - & - \\
\hline Globobulimina galliheri & - & - & - & - & - & $\mathrm{x}$ & - & - & - & - & - & - \\
\hline Haplophragmoides spp. & $\mathrm{x}$ & - & - & - & - & - & - & - & - & - & - & - \\
\hline Marginulina beali & - & - & - & - & - & $\mathrm{x}$ & - & - & - & - & - & - \\
\hline Nonionella costifera & $\mathrm{x}$ & - & - & - & $\mathrm{x}$ & $\mathrm{x}$ & $\mathrm{x}$ & - & - & - & - & - \\
\hline Nonionella miocenica & - & - & - & $\mathrm{X}$ & - & $x$ & - & $\mathrm{x}$ & - & - & - & - \\
\hline Nonionella spp. & - & - & - & - & - & - & - & - & $\mathrm{x}$ & $\mathrm{x}$ & - & - \\
\hline Nodosaria spp. & - & - & - & - & - & - & - & - & - & - & - & - \\
\hline Nonion spp. & $\mathrm{x}$ & - & - & - & - & - & - & - & - & - & - & - \\
\hline Praeglobobulimina ovata & - & - & - & - & - & $x$ & - & - & - & - & - & - \\
\hline Pullenia miocenica & $\mathrm{x}$ & - & - & - & - & - & - & - & - & - & - & - \\
\hline Siphogenerina branneri & $\mathrm{x}$ & - & $\mathrm{x}$ & - & - & $x$ & - & - & - & - & - & - \\
\hline Uvigerinella obesa & $\mathrm{x}$ & - & - & - & - & - & - & - & - & - & - & - \\
\hline Valvulineria californica & $\mathrm{X}$ & - & - & - & - & - & - & - & - & - & - & - \\
\hline
\end{tabular}


Table 14. Benthic foraminifers from unit Ku of Graymer and others (1996).

[Taxonomy of species is given in the section entitled "Taxonomic Notes." X indicates the species is present; cf. indicates that the specimens resemble or compare to the species. Samples that are barren or do not contain diagnostic microfossils include: Mf3335, Mf3336, and Mf3338]

\begin{tabular}{|c|c|c|}
\hline $\begin{array}{l}\text { Benthic foraminifers from unit Ku of } \\
\text { Graymer and others (1996) in Block II }\end{array}$ & $\underset{\stackrel{N}{N}}{\stackrel{N}{\Sigma}}$ & $\begin{array}{l}\hat{\mathscr{ల}} \\
\stackrel{ल}{\Sigma} \\
\frac{+}{\Sigma}\end{array}$ \\
\hline Bolivina argentea & $x$ & - \\
\hline Bolivina subadvena sulferensis & cf. & - \\
\hline Cassidulina californica & $x$ & - \\
\hline Cassidulina transluscens & $x$ & - \\
\hline Cassidulinoides cornuta & $x$ & - \\
\hline Cibicides fletcheri & $x$ & - \\
\hline Cibicides mckannai & $x$ & - \\
\hline Epistominella bradyana & $x$ & - \\
\hline Epistominella smithi & $x$ & - \\
\hline Fursenkoina bramletti & $x$ & - \\
\hline Gaudryina arenaria & $x$ & - \\
\hline Hanzawaia illingi & $x$ & - \\
\hline Lagena spp. & $x$ & - \\
\hline Lenticulina simplex & - & $x$ \\
\hline Nodosaria longiscata & $x$ & - \\
\hline Nodosaria spp. & - & $x$ \\
\hline Nonion spp. & - & $x$ \\
\hline ?Nonionella costifera & $x$ & - \\
\hline Nonionella miocenica & $x$ & - \\
\hline Oridorsalis tenera & $x$ & - \\
\hline Praeglobobulimina affinis & $x$ & - \\
\hline Pullenia malkinae & $x$ & - \\
\hline Rosalina columbiensis & cf. & - \\
\hline Siphonodosaria spp. & $x$ & - \\
\hline Stilostomella spp. & - & $x$ \\
\hline Trifarina angulosa & $x$ & - \\
\hline Valvulineria araucana & $x$ & - \\
\hline
\end{tabular}

\section{Block III}

Block III is a small block bounded by the Wildcat Fault to the west, the Pinole Fault to the east, San Pablo Bay to the north, and an unnamed fault to the south. This block, which is sandwiched between the northern parts of Blocks I and II, corresponds to a small portion of the East Bay Hills block (Buising and Walker, 1995) and the northwest portion of the Lafayette block (Graham and others, 1984). Graymer (2000) suggests that this block is an offset part of Block II based on the presence of correlative upper Miocene sedimentary rocks and tuff. Only Neogene strata were recognized by Graymer and others (1994a) in Block III, but a small sliver of previously unrecognized Paleogene strata was sampled during this study (fig. 9).

Three samples (Mf1021, Mf1101, Mf1102) from strata identified as unit Tmu (Graymer and others, 1994a) contain benthic foraminifers diagnostic of the early Eocene, late Penutian to early Ulatisian Stages coeval with planktic zones P8 and P9. Although these samples were collected from strata previously identified as Miocene (Graymer, 2000), the sample locality is close to a fault internal to Block III, and suggests a previously unrecognized fault sliver of older strata. These three samples are 
therefore considered to represent unit Tshc of Graymer and others (1994b, 1996). The presence of unit Tshc within Block III supports previous correlations of this block with Block II (Graymer, oral commun., 2010). Species diagnostic of an early Eocene age include Bulimina macilenta, Cibicidoides eocaenus, C. praemundulus, Uvigerina lodoensis mirmirae, and Vaginulinopsis asperuliformis (table 15). Deposition occurred at lower middle to lower bathyal depths (1,500-2,000 m).

Hill (1983) used formal stratigraphic nomenclature for the Neogene strata in Block III, recognizing the Tice Shale, Hambre Sandstone, Rodeo Shale, and Briones Sandstone, whereas Graymer and others (1994a) chose to use informal unit names (Tmu, Tsa, Tdi, Tut, and Tcgl) until the stratigraphic nomenclature could be resolved (fig. 9). Microfossil samples taken from the mudstone, shale, and siltstone unit (Tmu) and the diatomite unit (Tdi) are middle to late Miocene in age. No microfossil samples were examined from the lower sandstone (unit Tsa), the tuffaceous sandstone (unit Tut) or the conglomerate (unit Tcgl). The conglomerate unit (Tcgl) includes a tuff that is correlated with the Roblar tuff of Sarna-Wojcicki (1992) in Sonoma County and thus is considered to be late Miocene in age (6.25 Ma, Sarna-Wojcicki, 1992; 6.1-5.7 Ma, Graymer and others, 1994a).

Samples Mf7763 and Mf7764 from unit Tmu of Graymer and others (1994a) contain a moderately diverse benthic foraminiferal assemblage that is middle Miocene in age based on the presence of Bolivina advena straitella, Marginulina beali, Valvulineria californica, and V. miocenica (table 16). The overlapping ranges of these species are diagnostic of the Luisian Stage (probably early Luisian Stage). Although species with upper bathyal depth limits dominate, the presence of Bolivina salinasensis, Bolivina imbricata, and Epistominella cf. E. subperuviana indicate that deposition occurred at upper middle bathyal depths (500-1,500 m).

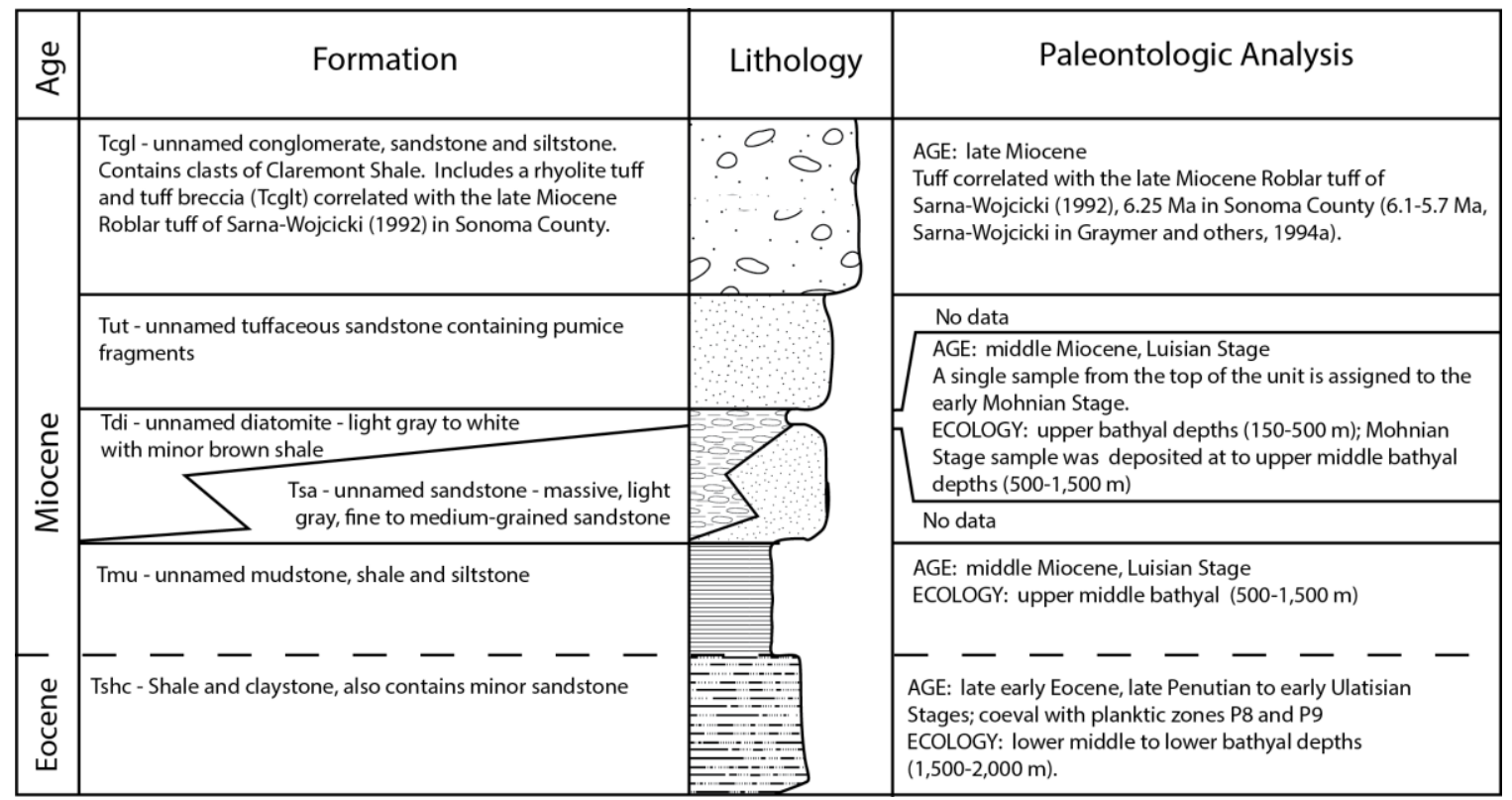

Figure 9. Composite columnar section for Block III. The nature of the Eocene/Miocene contact is unknown and shown with a dashed line. Age and environmental interpretations are summarized from the text. 
Table 15. Table 15. Benthic foraminifers in unit Tshc of Graymer and others (1994a, 1996) in Block III.

[Taxonomy of species is given in section entitled "Taxonomic Notes." $\mathrm{X}$ indicates that the species is present; cf. indicates that the specimens resemble or compare to the species]

\begin{tabular}{|c|c|c|c|}
\hline $\begin{array}{c}\text { Benthic foraminifers in unit Tshc of } \\
\text { Graymer and others (1994a, 1996) in } \\
\text { Block III }\end{array}$ & 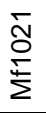 & $\frac{5}{\frac{5}{5}}$ & 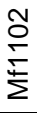 \\
\hline Allomorphina conica & - & $X$ & $x$ \\
\hline Amphimorphina ignota & - & $x$ & - \\
\hline Anomalina regina & $\mathrm{X}$ & $X$ & $x$ \\
\hline Anomalinoides capitatus & $X$ & $x$ & - \\
\hline Aragonia aragonensis & $X$ & $X$ & - \\
\hline Bathysiphon spp. & - & - & $X$ \\
\hline Bolivina explicata lodoensis & $\mathrm{X}$ & - & $\mathrm{X}$ \\
\hline Bulimina alazaensis & $x$ & - & $\mathrm{X}$ \\
\hline Bulimina callahani & - & $\mathrm{X}$ & $\mathrm{X}$ \\
\hline Bulimina elongata & - & - & $\mathrm{X}$ \\
\hline Bulimina macilenta & $x$ & $\mathrm{X}$ & $\mathrm{X}$ \\
\hline Bulimina trinitatensis & - & $x$ & - \\
\hline Chilostomella cylindroides & - & $\mathrm{X}$ & - \\
\hline Chilostomella oolina & - & $\mathrm{X}$ & $\mathrm{X}$ \\
\hline Chrysalongonium elongatum & - & $X$ & - \\
\hline Cibicides spiropunctatus & $x$ & - & - \\
\hline Cibicidoides fortunatus & $x$ & - & - \\
\hline Cibicidoides eocaenus & - & $\mathrm{X}$ & $\mathrm{X}$ \\
\hline Cibicidoides eponidiformis & - & $\mathrm{X}$ & $\mathrm{X}$ \\
\hline Cibicidoides pachecoensis & - & $x$ & $\mathrm{X}$ \\
\hline Cibicidoides praemundulus & - & $X$ & - \\
\hline Cibicidoides subspiratus & - & - & $\mathrm{X}$ \\
\hline Cibicidoides venzuelanus & - & $X$ & $\mathrm{X}$ \\
\hline Clavulina anglica & - & $X$ & - \\
\hline Cyclammina simiensis & - & - & $\mathrm{X}$ \\
\hline Dentalina approximata & $X$ & - & - \\
\hline Dentalina basispinata & $\mathrm{X}$ & - & - \\
\hline Dentalina colei & $X$ & - & - \\
\hline Dentalina communis & - & $x$ & $X$ \\
\hline Dentalina consobrina & $X$ & $\mathrm{X}$ & - \\
\hline Dentalina significa & $x$ & - & - \\
\hline Dentalina substrigata & $\mathrm{X}$ & - & - \\
\hline Dentalina spp. & & - & $\mathrm{X}$ \\
\hline Dorothia principiensis & $\mathrm{X}$ & $\mathrm{X}$ & - \\
\hline Fissurina marginata & - & - & $\mathrm{X}$ \\
\hline Fissurina orbignyana & - & - & $X$ \\
\hline Gaudryina laevigata & - & $X$ & $\mathrm{X}$ \\
\hline Globocassidulina globosa & - & - & $X$ \\
\hline Globulina spp. & - & - & $\mathrm{X}$ \\
\hline Gyroidina octocameratus & - & $X$ & $\mathrm{X}$ \\
\hline Gyroidina soldanii & - & $X$ & - \\
\hline Hanzawaia mauriciensis & - & - & $\mathrm{X}$ \\
\hline Haplophragmoides eggeri & - & $x$ & $\mathrm{X}$ \\
\hline Haplophragmoides sp. & $X$ & - & - \\
\hline Karreriella conversa & - & - & $\mathrm{X}$ \\
\hline Lagena conscripta & $X$ & - & - \\
\hline Lagena spp. & - & - & $\mathrm{X}$ \\
\hline Lenticulina altolimbatus & - & - & $\mathrm{x}$ \\
\hline Lenticulina midwayensis & $\mathrm{x}$ & - & - \\
\hline
\end{tabular}

\begin{tabular}{|c|c|c|c|}
\hline $\begin{array}{c}\text { Benthic foraminifers in unit Tshc of } \\
\text { Graymer and others (1994a, 1996) in } \\
\text { Block III }\end{array}$ & సัণ & $\stackrel{\overline{0}}{\sum_{\Sigma}^{E}}$ & 옹 \\
\hline Lenticulina pseudovortex & $\mathrm{X}$ & - & $x$ \\
\hline Lenticulina spp. & - & $\mathrm{X}$ & - \\
\hline Lenticulina texana & - & $\mathrm{X}$ & - \\
\hline Lenticulina theta & $X$ & - & - \\
\hline Lenticulina turbinatus & - & - & $x$ \\
\hline Loxostomoides applinae & $\mathrm{X}$ & $\mathrm{X}$ & - \\
\hline Marginulina hunneri & $\mathrm{X}$ & - & - \\
\hline Marginulina subbullata & $\mathrm{X}$ & $\mathrm{X}$ & - \\
\hline Nodosaria delicata & $\mathrm{X}$ & $\mathrm{X}$ & $X$ \\
\hline Nodosaria gyrata & $\mathrm{X}$ & - & - \\
\hline Nodosaria latejugata & $x$ & $\mathrm{X}$ & - \\
\hline Nodosaria longiscata & - & $\mathrm{X}$ & $\mathrm{X}$ \\
\hline Nodosaria raphanistrum caribbeana & - & $\mathrm{X}$ & - \\
\hline Nodosaria spp. & - & $\mathrm{X}$ & - \\
\hline Nodosaria velascoensis & $\mathrm{X}$ & - & - \\
\hline Nodosarella constricta & - & $\mathrm{X}$ & - \\
\hline Nuttaloides truempyi & - & $X$ & - \\
\hline Oridorsalis umbonatus & - & $\mathrm{X}$ & $X$ \\
\hline Osangularia midwayensis & $\mathrm{X}$ & - & - \\
\hline Osangularia mexicana & - & $\mathrm{X}$ & $X$ \\
\hline Plectofrondicularia paucicostata & $\mathrm{X}$ & - & - \\
\hline Plectofrondicularia spp. & $\mathrm{X}$ & - & - \\
\hline Praeglobobulimina pupoides & - & $\mathrm{X}$ & $\mathrm{X}$ \\
\hline Pullenia spp. & - & $\mathrm{X}$ & - \\
\hline Pyrulina cylindroides & - & $\mathrm{X}$ & - \\
\hline Quadrimorphina allomorphinoides & - & $X$ & - \\
\hline Rhabdammina eocenica & $X$ & - & - \\
\hline Saracenaria spp. & - & $X$ & $X$ \\
\hline Siphonia wilcoxensis & $\mathrm{X}$ & - & - \\
\hline Silicosigmoilina californica & - & $\mathrm{X}$ & - \\
\hline Spiroloculina texana & - & - & $X$ \\
\hline Spiroplectammina directa & $x$ & - & $\mathrm{X}$ \\
\hline Spiroplectammina richardi & $X$ & $X$ & $X$ \\
\hline Stilostomella adolphina & - & $\mathrm{X}$ & $X$ \\
\hline Stilostomella lepidula & - & $\mathrm{X}$ & $X$ \\
\hline Stilostomella spp. & - & $\mathrm{X}$ & - \\
\hline Textularia adalta & - & $\mathrm{X}$ & - \\
\hline Trifarina advena californica & $\mathrm{X}$ & $\mathrm{X}$ & $\mathrm{X}$ \\
\hline Tritaxilina colei & $\mathrm{X}$ & $\mathrm{X}$ & - \\
\hline Uvigerina alabamensis & $X$ & - & - \\
\hline Uvigerina elongata & - & - & $X$ \\
\hline Uvigerina lodoensis miriamae & - & $X$ & $X$ \\
\hline Vaginulinopsis asperuliformis & $\mathrm{X}$ & $X$ & - \\
\hline Vaginulinopsis echinata & $\mathrm{X}$ & - & - \\
\hline Valvulineria jacksonensis welcomensis & - & cf. & - \\
\hline Vaginulinopsis nudicostata & $X$ & - & - \\
\hline Valvulineria spp. & $X$ & - & - \\
\hline Valvulineria wilcoxensis & - & - & $\mathrm{X}$ \\
\hline Verneuilina triangulata & $\mathrm{X}$ & - & - \\
\hline
\end{tabular}


Table 16. Benthic foraminifers from the Neogene of Block III.

[Taxonomy of species is given in the section entitled "Taxonomic Notes". $\mathrm{X}$ indicates the species is present. Samples that are barren of foraminifers include: Mf7848, Mf7849, Mf7850, and Mf7851]

\begin{tabular}{|c|c|c|c|c|c|c|c|c|c|}
\hline \multirow[b]{2}{*}{$\begin{array}{l}\text { Benthic foraminifers from the } \\
\text { Neogene of Block III }\end{array}$} & \multicolumn{2}{|c|}{ Tmu } & \multicolumn{6}{|c|}{ Tdi } & \multirow{2}{*}{$\begin{array}{l}\text { Tdi } \\
? \\
\text { ? } \\
\text { N } \\
\frac{T}{\Sigma}\end{array}$} \\
\hline & $\begin{array}{l}\text { D } \\
\stackrel{2}{N} \\
\stackrel{1}{\Sigma}\end{array}$ & 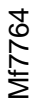 & $\begin{array}{l}\infty \\
\infty \\
\infty \\
\stackrel{\infty}{\Sigma}\end{array}$ & $\begin{array}{l}\mathscr{Q} \\
\infty \\
\infty \\
\stackrel{0}{\Sigma}\end{array}$ & 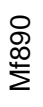 & $\begin{array}{l}\bar{\delta} \\
\text { क्र } \\
\sum\end{array}$ & $\begin{array}{l}\text { న్ } \\
\text { o } \\
\sum\end{array}$ & 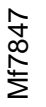 & \\
\hline Bolivina advena ornata & - & - & - & - & - & - & - & $x$ & - \\
\hline Bolivina advena striatella & $x$ & $x$ & - & $x$ & - & $x$ & - & - & $x$ \\
\hline Bolivina guadeloupae & - & - & $x$ & - & - & - & - & - & $x$ \\
\hline Bolivina imbricata & $x$ & $x$ & $x$ & - & - & - & - & - & - \\
\hline Bolivina marginata & - & $\mathrm{X}$ & - & - & - & - & $\mathrm{X}$ & - & - \\
\hline Bolivina salinensis & $x$ & $x$ & - & - & - & - & - & - & - \\
\hline Bolivina spp. & - & - & - & - & $X$ & - & - & - & - \\
\hline Buccella frigida & - & - & $\mathrm{x}$ & - & - & - & - & - & - \\
\hline Buliminella curta & - & - & - & - & - & $x$ & $x$ & $x$ & - \\
\hline Buliminella subfusiformis & $x$ & $x$ & - & - & - & - & $x$ & $x$ & $x$ \\
\hline Cassidulina panzana & - & - & - & - & - & - & - & $x$ & - \\
\hline Cassidulina puchella & $x$ & - & - & - & - & - & - & - & - \\
\hline Concavella gyroidinaformis & - & - & - & - & - & - & - & - & $x$ \\
\hline Dentalina spp. & $x$ & - & - & - & - & - & - & - & - \\
\hline Epistominella relizensis & cf. & - & - & - & - & - & - & - & - \\
\hline Fursenkoina californiensis & $x$ & $x$ & - & - & - & $\mathrm{X}$ & $x$ & - & - \\
\hline Fursenkoina californiensis ticensis & - & - & - & - & - & - & - & - & $x$ \\
\hline Lenticulina spp. & $x$ & - & - & - & - & - & - & - & - \\
\hline Marginulina beali & $x$ & - & - & - & - & - & - & - & - \\
\hline Nonionella costifera & $x$ & - & $x$ & $\mathrm{X}$ & - & $x$ & $x$ & $x$ & - \\
\hline Nonionella miocenica & - & - & - & - & - & - & - & - & $x$ \\
\hline Nonionella spp. & - & $X$ & - & - & - & - & - & - & - \\
\hline Plectofrondicularia spp. & - & - & - & - & $\mathrm{X}$ & - & - & - & - \\
\hline Uvigerinella obesa & - & - & - & - & - & - & $x$ & - & - \\
\hline Valvulineria araucana & - & - & - & - & - & - & - & - & $x$ \\
\hline Valvulineria californica s.s. & $x$ & $x$ & - & $x$ & $x$ & $x$ & - & $x$ & - \\
\hline Valvulineria miocenica & $x$ & - & - & - & - & - & - & - & - \\
\hline Valvulineria spp. & $x$ & - & - & - & - & - & - & - & - \\
\hline
\end{tabular}

\section{Block IV}

The triangular Block IV is bounded by the Carquinez Strait to the north, the Franklin Fault to the west, and the Calaveras Fault to the east (fig. 1). The Tertiary section rests unconformably on the Cretaceous Great Valley sequence. This block corresponds to a portion of the East Bay Hills block (Buising and Walker, 1995) and the northern portion of the Lafayette block (Graham and others, 1984). The Paleogene units include the Vine Hill Sandstone, Las Juntas Shale, Muir Sandstone, Escobar Sandstone, and Alhambra Formation. Neogene units from this block include the San Ramon and Sobrante Sandstones, an unnamed conglomerate (unit Tuc), and an unnamed shale (unit Tchs) (Graymer and others, 1994a; Graymer, 2000). The Paleogene formations were sampled at Selby Point, Pacheco syncline, and near Walnut Creek. New samples from the Selby Point and Pacheco syncline sections of this study are combined with samples from Smith (1957) (fig. 10). Three samples were taken near Walnut Creek (Mf1738, Mf1739, and Mf8542), from a Neogene unit (Tchs) of Block IV, but no microfossil data are available for these samples. 


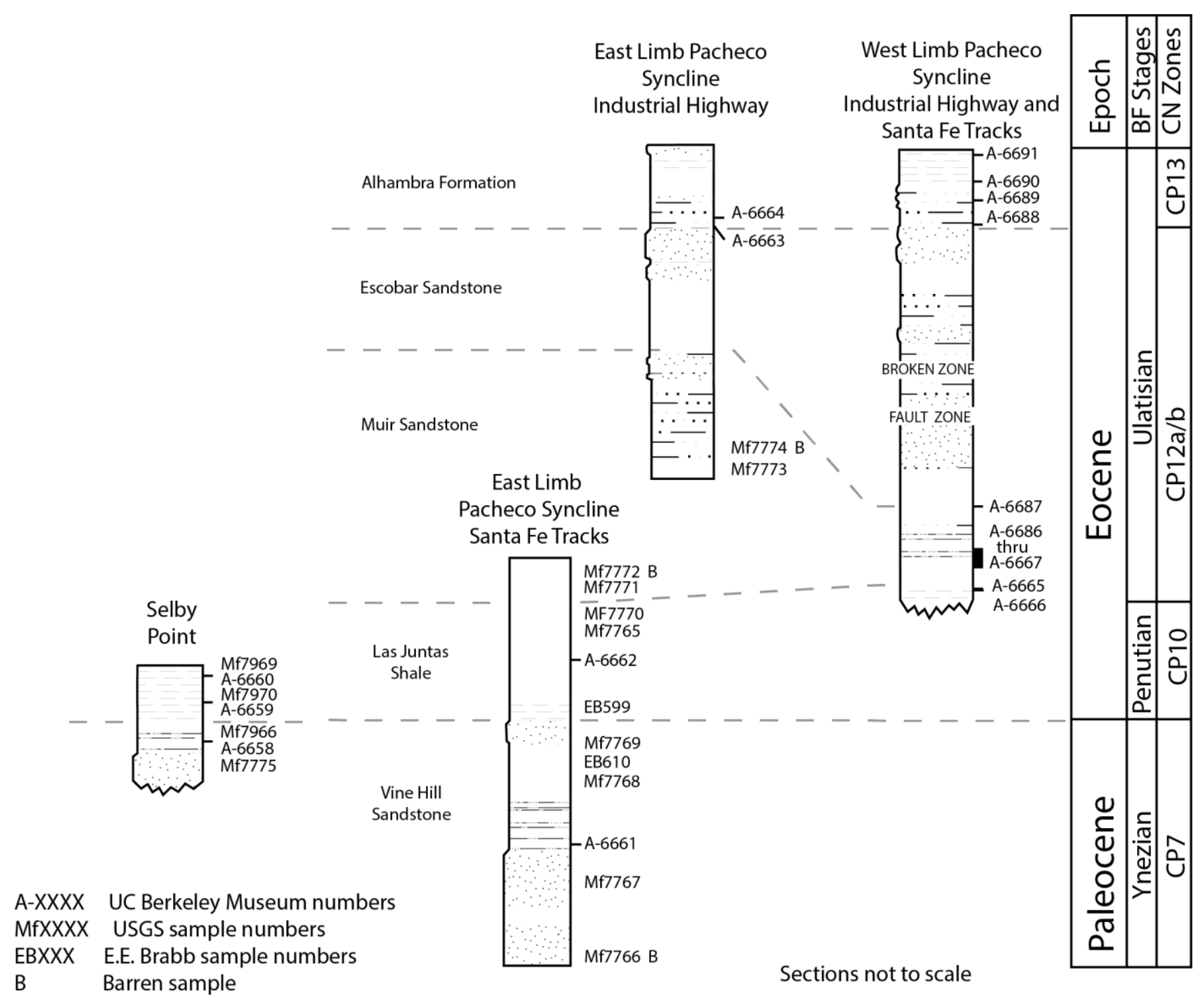

Figure 10. Correlation of Pacheco syncline and Selby Point sections. Modified from Smith (1957).

Two new samples, Mf7775 and Mf7966, were taken from the Vine Hill Sandstone (Weaver, 1953) in the Selby Point section (fig. 11; table 17). Sample Mf7775 contains only a few nondiagnostic arenaceous benthic foraminifers, whereas samples Mf7966 and A6658 (Smith, 1957) from approximately the same location contain a diverse benthic foraminiferal assemblage that is late Paleocene in age and assigned to the Ynezian Stage, coeval with planktic foraminiferal zone P4. This age is based on numerous diagnostic cosmopolitan species such as Anomalinoides acutus, A. rubiginosus, Cibicidoides alleni, C. dayi, and Frondicularia frankei, as well as U.S. West Coast and Gulf Coast species such as Ammobacculites paleocenica, Bulimina cacumenata, Citharina plummoides, Clavulinoides midwayensis, Coleites recticulosus, Lenticulina midwayensis, Osangularia midwayensis, Pseudoglandulina manifesta, Uvigerina laimingi, and Vaginulinopsis tuberculata. Calcareous nannofossils from these samples indicate a late Paleocene age and are assigned to zone CP7 (Bukry and others, 1977, 1998; Poore, 1976), supporting the foraminiferal age interpretation. 


\begin{tabular}{|c|c|c|c|c|}
\hline gั & Formation & \multicolumn{2}{|c|}{ Lithology } & Paleontologic Analysis \\
\hline 岂 & $\begin{array}{l}\text { Las Juntas Shale (Tlj) - Gray shale with } \\
\text { minor siltstone. Locally divided into the } \\
\text { upper shale member (Tlju) and lower } \\
\text { sandstone member (Tlj) (Weaver, 1953; } \\
\text { Graymer and others, 1994a) }\end{array}$ & 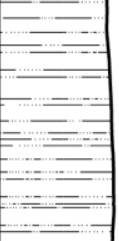 & \multirow{3}{*}{ Fault } & $\begin{array}{l}\text { AGE: early Eocene, Penutian Stage coeval with planktic } \\
\text { foraminiferal zones P6b-P9 } \\
\text { ECOLOGY: middle bathyal depths }(500-2,000 \mathrm{~m})\end{array}$ \\
\hline 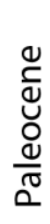 & $\begin{array}{l}\text { Vine Hill Sandstone (Tvh) - Glauconitic } \\
\text { sandstone locally divided (Weaver, 1953; } \\
\text { Graymer and others, 1994a) }\end{array}$ & $\begin{array}{c}= \\
- \\
- \\
-\end{array}$ & & $\begin{array}{l}\text { AGE: late Paleocene, Ynezian Stage; calcareous nannofossil } \\
\text { zone CP7 (Poore, 1976; Bukry and others, 1977; Bukry pers. } \\
\text { com., 1996) } \\
\text { ECOLOGY: middle bathyal depths }(500-2,000 \mathrm{~m})\end{array}$ \\
\hline 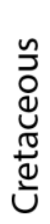 & $\begin{array}{l}\text { Great Valley sequence - sandstone, } \\
\text { siltstone, shale, and minor conglomerate. }\end{array}$ & & & \\
\hline
\end{tabular}

Figure 11. Composite columnar section for Block IV, Selby Point section. Age and environmental interpretations are summarized from the text.

The Vine Hill Sandstone (Weaver, 1953) was also sampled along the Santa Fe Railroad tracks on the east limb of the Pacheco syncline (figs. 10, 12; table 17). Benthic foraminiferal assemblages include numerous age diagnostic cosmopolitan species such as Anomalinoides rubiginosus, Cibicidoides alleni, and C. dayi as well as U.S. West Coast and Gulf Coast species such as Ammobacculites paleocenica, Bulimina cacumenata, Citharina plummeroides, Clavulina midwayensis, Coleites recticulosus, Frondicularia frankei, Gyroidina obliquata, Lenticulina midwayensis, Osangularia midwayensis, Palmula primitiva, Pseudoglandulina manifesta, Uvigerina laimingi, Vaginulinopsis earlandi, and $V$. tuberculata. Together these species suggest a Paleocene age and are assigned to the Ynezian Stage coeval with planktic foraminiferal zone P4, which is supported by calcareous nannofossils that indicate a late Paleocene age and are assigned to zone CP7 (Bukry and others, 1977, 1998; Poore, 1976).

Samples from the Vine Hill Sandstone (Weaver, 1953) near Walnut Creek (table 17) are also Paleocene in age based on the presence of Anomalinoides praeacutus, A. rubiginosus, Bulimina midwayensis, B. quadrata, Chilostomella eocenica, Clavulina midwayensis, Dorothia retusa, Eponides plummerae, Lenticulina pseudomammillerga, Marssonella oxycona, and Stensioina beccariformis as well as species that range from the Paleocene into the Eocene such as Anomalinoides welleri and Silicosigmoilina californica. This assemblage is assigned to the late Paleocene, Ynezian Stage coeval with planktic foraminiferal zone P4. 
Table 17. Benthic foraminifers from the Vine Hill Sandstone in Block IV.

[Taxonomy of species is given in the section entitled "Taxonomic Notes." $\mathrm{X}$ indicates the species is present; cf. indicates that the specimens resemble or compare to the species; aff. indicates that the specimens are related to but not identical with the species. Samples that are barren of microfossils or contain few to rare nondiagnostic species include: Mf7779, Mf7780, and Mf8787. Sample Mf7776 mapped as Vine Hill Sandstone yields a late Campanian age]

\begin{tabular}{|c|c|c|c|c|c|c|c|c|c|c|c|c|c|c|c|}
\hline \multirow[b]{2}{*}{$\begin{array}{l}\text { Benthic foraminifers from the } \\
\text { Vine Hill Sandstone in Block } \\
\text { IV }\end{array}$} & \multicolumn{3}{|c|}{ Selby Point } & \multicolumn{5}{|c|}{ Pacheco syncline } & \multicolumn{7}{|c|}{ Walnut Creek } \\
\hline & $\stackrel{\substack{N \\
N}}{N}$ & 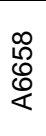 & 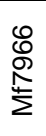 & \begin{tabular}{l}
$\hat{0}$ \\
$\hat{N}$ \\
\multirow{2}{*}{} \\
$\Sigma$
\end{tabular} & $\begin{array}{l}\overline{6} \\
0 \\
0\end{array}$ & 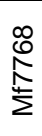 & $\begin{array}{l}\frac{*}{0} \\
6 \\
\frac{1}{5} \\
\frac{1}{\Sigma}\end{array}$ & $\frac{\substack{0 \\
N}}{\stackrel{N}{\Sigma}}$ & $\begin{array}{l}\infty \\
\stackrel{\infty}{\infty} \\
\stackrel{1}{\Sigma} \\
\sum\end{array}$ & $\begin{array}{l}\infty \\
1 \\
\infty \\
\\
\Sigma\end{array}$ & \begin{tabular}{l}
0 \\
$\infty$ \\
$\infty$ \\
\multirow{1}{*}{}
\end{tabular} & $\begin{array}{l}\frac{1}{N} \\
\infty \\
\stackrel{N}{N} \\
\sum\end{array}$ & 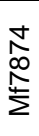 & 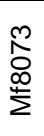 & $\begin{array}{l}\text { N } \\
\text { O } \\
\stackrel{\infty}{\Sigma} \\
\sum\end{array}$ \\
\hline Alabamina wilcoxensis & - & $\mathrm{X}$ & $\mathrm{X}$ & - & - & - & $\mathrm{X}$ & - & - & - & - & - & - & - & - \\
\hline Allomorphina conica & - & $X$ & - & - & - & - & - & - & - & - & - & - & - & - & - \\
\hline Allomorphina halli & - & $X$ & - & - & $X$ & - & - & - & - & - & - & - & - & - & - \\
\hline Ammobacculites expansus & - & $X$ & - & - & - & - & $X$ & - & - & - & - & - & - & - & - \\
\hline Ammobacculites paleocenica & - & - & $X$ & $X$ & - & - & $X$ & - & - & - & - & $X$ & - & - & - \\
\hline Ammodiscus incertus & - & - & - & - & - & - & - & - & $x$ & $X$ & - & - & - & - & - \\
\hline Ammodiscus pennyi & - & - & - & - & - & - & - & - & - & - & - & $X$ & - & - & - \\
\hline Anomalina madrugaensis & - & - & $X$ & - & - & - & - & - & - & - & - & - & - & - & - \\
\hline Anomalina keenae & - & - & - & - & - & - & - & - & - & - & - & - & - & $X$ & - \\
\hline Anomalina regina & - & - & - & cf. & - & - & - & - & - & - & - & - & - & $X$ & - \\
\hline Anomalinoides acutus & - & $X$ & - & $X$ & $X$ & - & $X$ & - & - & - & - & - & - & - & - \\
\hline Anomalinoides capitatus & - & $X$ & - & $X$ & $X$ & - & $X$ & - & - & - & - & - & - & - & - \\
\hline Anomalinoides midwayensis & - & $X$ & $X$ & - & - & - & $X$ & - & - & - & - & - & - & - & - \\
\hline Anomalinoides praeacuta & - & - & - & - & - & - & - & - & - & - & $X$ & - & - & - & - \\
\hline Anomalinoides rubiginosus & - & - & $X$ & - & - & - & - & - & - & - & $X$ & - & $\mathrm{X}$ & - & - \\
\hline Anomalinoides welleri & - & - & $X$ & - & - & - & - & - & - & - & $X$ & - & - & - & - \\
\hline Bathysiphon eocenicus & - & $X$ & $X$ & - & - & $X$ & $X$ & $X$ & - & $X$ & - & - & $X$ & $X$ & $X$ \\
\hline Bathysiphon santecruis & - & - & - & - & - & - & - & - & - & - & - & $X$ & $X$ & - & $X$ \\
\hline Bathysiphon spp. & $X$ & - & $X$ & - & - & - & $X$ & $X$ & $x$ & $X$ & $X$ & $X$ & - & $X$ & - \\
\hline Bulimina cacumenata & - & $X$ & - & $x$ & $X$ & - & $X$ & - & - & - & - & - & - & - & - \\
\hline Bulimina macilenta & - & - & $X$ & $X$ & $X$ & - & $X$ & - & - & - & - & - & - & $X$ & - \\
\hline Bulimina midwayensis & - & - & - & - & - & - & - & - & - & - & $X$ & - & - & - & - \\
\hline Bulimina quadrata & - & - & - & - & - & - & - & - & - & - & $X$ & - & - & - & - \\
\hline Bulimina spp. & - & - & $X$ & - & - & - & - & - & - & - & - & - & - & - & - \\
\hline Ceratobulimina perplexa & - & - & - & - & - & - & $X$ & - & - & - & - & - & - & - & - \\
\hline Chilostomella oolina & - & - & - & - & - & - & - & - & - & - & - & $X$ & - & - & - \\
\hline Chrysalongonium elongatum & - & - & - & - & - & - & - & - & - & - & $X$ & - & - & - & - \\
\hline Chrysalongonium teniucostatum & - & - & - & - & - & - & - & - & - & - & $X$ & - & - & - & - \\
\hline Cibicides pachecoensis & - & $X$ & - & - & - & - & $X$ & - & - & - & - & - & - & - & - \\
\hline Cibicidoides alleni & - & - & $X$ & - & - & - & - & - & - & - & - & - & - & - & - \\
\hline Cibicidoides dayi & - & - & $X$ & - & - & - & - & - & - & - & - & - & - & - & - \\
\hline Cibicidoides eocaenus & - & - & - & - & - & - & $X$ & - & - & - & cf. & - & - & $X$ & - \\
\hline Cibicidoides eponidiformis & - & $X$ & $X$ & $\mathrm{X}$ & $X$ & - & $X$ & - & - & - & - & - & - & $X$ & - \\
\hline Cibicidoides subspiratus & - & - & - & - & - & - & - & - & - & - & - & $X$ & - & $X$ & - \\
\hline Cibicidoides spp. & - & - & - & $X$ & - & - & - & - & - & - & - & - & - & - & - \\
\hline Cibicidoides venzuelanus & - & - & - & - & - & - & - & - & - & - & - & - & - & $X$ & - \\
\hline Citharina plummoides & - & $X$ & $X$ & - & $X$ & - & $X$ & - & - & - & - & - & - & - & - \\
\hline Clavulinoides midwayensis & - & $X$ & $X$ & $X$ & $X$ & - & $X$ & - & - & - & $X$ & $X$ & $X$ & - & - \\
\hline Coleites reticulosus & - & $X$ & - & - & - & - & - & - & - & - & - & - & - & - & - \\
\hline Coryphostoma midwayensis & - & $X$ & - & - & - & - & - & - & - & - & - & - & - & - & - \\
\hline Cribrostomoides cretacea & - & - & - & - & - & - & - & - & - & - & - & $X$ & $\mathrm{X}$ & - & - \\
\hline Cyclammina clarki & - & - & - & - & - & - & $X$ & - & - & - & - & - & - & - & - \\
\hline Cyclammina samanica & - & - & - & - & - & - & - & - & $X$ & - & - & - & - & - & - \\
\hline Cyclammina simiensis & - & - & - & - & - & $X$ & $X$ & - & - & - & - & - & - & - & - \\
\hline Cyclammina simiensis & - & - & - & - & - & cf. & - & - & - & - & - & - & - & - & - \\
\hline Cyclammina spp. & - & - & - & - & - & - & - & $X$ & - & - & - & $X$ & - & - & - \\
\hline Dentalina colei & - & $X$ & $X$ & - & - & - & - & - & - & - & - & - & - & $X$ & - \\
\hline Dentalina communis & - & - & - & - & - & - & - & - & - & - & $X$ & - & - & $X$ & - \\
\hline Dentalina consobrina & - & $X$ & - & - & - & - & - & - & - & - & - & - & - & - & - \\
\hline Dentalina hexacostata & - & - & - & - & - & - & - & - & - & - & - & - & - & - & $X$ \\
\hline Dentalina insulsa & - & - & - & - & - & - & - & - & - & - & $X$ & - & - & - & - \\
\hline Dentalina jacksonensis & - & - & - & - & - & - & - & - & - & - & - & - & - & $X$ & - \\
\hline Dentalina pseudobliquestriata & - & - & $X$ & - & - & - & $X$ & - & - & - & - & - & - & - & - \\
\hline Dentalina spinosa & - & $X$ & - & - & - & - & - & - & - & - & - & - & - & - & - \\
\hline Dentalina spp. & - & $X$ & - & - & $X$ & - & - & - & - & - & - & - & - & - & - \\
\hline Dorothia principiensis & - & - & - & - & - & - & $X$ & - & - & - & - & - & - & - & - \\
\hline
\end{tabular}




\begin{tabular}{|c|c|c|c|c|c|c|c|c|c|c|c|c|c|c|c|}
\hline \multirow[b]{2}{*}{$\begin{array}{l}\text { Benthic foraminifers from the } \\
\text { Vine Hill Sandstone in Block } \\
\text { IV }\end{array}$} & \multicolumn{3}{|c|}{ Selby Point } & \multicolumn{5}{|c|}{ Pacheco syncline } & \multicolumn{7}{|c|}{ Walnut Creek } \\
\hline & $\stackrel{\substack{N \\
N}}{N}$ & 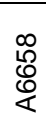 & 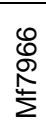 & $\frac{N}{N}$ & $\begin{array}{l}\bar{\emptyset} \\
\bigoplus \\
\mathscr{\varangle}\end{array}$ & $\frac{\infty}{\substack{\infty \\
N}}$ & $\begin{array}{l}\frac{*}{6} \\
\frac{0}{5} \\
\sum_{\Sigma}^{\frac{L}{\Sigma}}\end{array}$ & 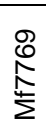 & $\begin{array}{l}\infty \\
\infty \\
\infty \\
1 \\
\sum \\
\Sigma\end{array}$ & $\begin{array}{l}\infty \\
\infty \\
\infty \\
\stackrel{N}{\Sigma}\end{array}$ & $\begin{array}{l}0 \\
\infty \\
\infty \\
⿱ 亠 䒑 \\
\Sigma\end{array}$ & $\begin{array}{l}\frac{1}{N} \\
\infty \\
\stackrel{N}{\Sigma} \\
\sum\end{array}$ & 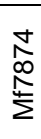 & $\begin{array}{l}\text { N } \\
\stackrel{\infty}{\infty} \\
\stackrel{\infty}{\Sigma}\end{array}$ & 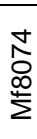 \\
\hline Dorothia retusa & - & - & - & - & - & - & - & - & - & - & - & $\mathrm{X}$ & - & - & - \\
\hline Ellipsonodosaria alexanderi & - & - & - & - & - & - & - & - & - & - & $X$ & - & - & - & - \\
\hline Eponides lodoensis & - & - & - & - & - & - & $X$ & - & - & - & - & - & - & $X$ & - \\
\hline Eponides mexicaunus & - & - & - & - & - & - & aff. & - & - & - & - & - & - & $X$ & - \\
\hline Eponides plummerae & - & - & - & - & - & - & - & - & - & - & - & $X$ & $\mathrm{X}$ & - & - \\
\hline Eponides spp. & - & - & - & - & - & - & $X$ & - & - & - & - & - & - & - & - \\
\hline Eponides waltonensis & - & $X$ & - & - & $X$ & - & $X$ & - & - & - & - & - & - & - & - \\
\hline Frondicularia frankei & - & $X$ & $X$ & $X$ & - & - & $X$ & - & - & - & - & - & - & - & - \\
\hline Gaudryina coalingensis & - & - & $X$ & $X$ & $X$ & - & $X$ & - & - & - & - & - & - & - & - \\
\hline Gaudryina pachecoensis & - & - & - & - & - & - & $X$ & - & - & - & - & - & - & - & - \\
\hline Gaudryina spp. & - & - & - & $X$ & - & - & - & - & - & - & - & - & - & - & - \\
\hline Globulina spp. & - & - & - & - & - & - & - & - & - & - & $X$ & - & $\mathrm{X}$ & $\mathrm{X}$ & - \\
\hline Guttulina irregularis & - & $X$ & - & - & $X$ & - & - & - & - & - & - & - & - & - & - \\
\hline Guttulina problema & - & - & - & - & - & - & - & - & - & - & $X$ & $X$ & $\mathrm{X}$ & - & - \\
\hline Gyroidina obliquata & - & - & - & $X$ & - & - & $X$ & - & - & - & - & - & - & - & - \\
\hline Gyroidina octocameratus & - & $X$ & - & - & $X$ & - & - & - & - & - & - & - & - & - & - \\
\hline Gyroidina soldanii & - & - & $X$ & - & - & - & $X$ & - & - & - & - & - & - & $X$ & - \\
\hline Haplophragmoides eggeri & - & - & - & - & - & $x$ & - & - & $X$ & $X$ & - & - & - & - & - \\
\hline Haplophragmoides spp. & $X$ & - & $X$ & - & - & $X$ & $X$ & $X$ & $X$ & $X$ & - & - & - & - & $X$ \\
\hline Hoeglundina eocenica & - & - & $X$ & - & - & - & $X$ & - & - & - & $X$ & - & $\mathrm{X}$ & - & - \\
\hline Karreriella conversa & - & - & - & - & - & - & - & - & - & $X$ & - & - & - & - & - \\
\hline Lagena costata & - & - & - & - & - & - & - & - & - & - & - & - & - & $X$ & - \\
\hline Lagena hexagona & - & - & - & - & $X$ & - & - & - & - & - & - & - & - & - & - \\
\hline Lagena spp. & - & $X$ & - & - & - & - & - & - & - & - & - & - & - & - & - \\
\hline Lagena striata & - & - & - & - & - & - & - & - & - & - & - & - & - & $\mathrm{X}$ & - \\
\hline Lenticulina altolimbatus & - & $\mathrm{X}$ & $\mathrm{X}$ & - & - & - & $X$ & - & - & - & - & - & - & - & - \\
\hline Lenticulina convergens & - & $X$ & - & - & - & - & - & - & - & - & - & - & - & - & - \\
\hline Lenticulina inornata & - & $X$ & - & - & $X$ & - & - & - & - & - & - & - & - & - & - \\
\hline Lenticulina midwayensis & - & - & $X$ & - & - & - & $X$ & - & - & - & - & - & - & - & - \\
\hline Lenticulina pseudomammiligera & - & - & - & - & - & - & - & - & - & - & $X$ & $X$ & $X$ & - & - \\
\hline Lenticulina pseudovortex & - & - & $X$ & - & - & - & $X$ & - & - & - & - & - & - & - & - \\
\hline Lenticulina rosettus & - & - & - & - & - & - & - & - & - & - & - & - & $X$ & - & - \\
\hline Lenticulina rotulata & - & - & - & - & - & - & - & - & - & - & $X$ & - & $\mathrm{X}$ & - & - \\
\hline Lenticulina spp. & - & - & $X$ & $X$ & - & - & $X$ & $X$ & - & - & $X$ & $X$ & $\mathrm{X}$ & $X$ & $X$ \\
\hline Lenticulina turbinatus & - & - & - & - & - & - & - & - & - & - & - & - & $\mathrm{X}$ & - & - \\
\hline Lenticulina vortex & - & $X$ & $X$ & $X$ & - & - & $X$ & - & - & - & - & - & - & - & - \\
\hline Lenticulina welchi & - & - & - & - & - & - & - & - & - & - & - & - & - & cf. & - \\
\hline Lenticulina williamsoni & - & cf. & - & - & - & - & - & - & - & - & - & - & - & - & - \\
\hline Loxostomoides applinae & - & - & $\mathrm{X}$ & - & - & - & - & - & - & - & - & - & - & - & - \\
\hline Marginulina spp. & - & - & $X$ & - & - & - & - & - & - & - & $X$ & - & - & - & - \\
\hline Marginulina subbullata & - & - & - & - & - & - & - & - & - & - & - & - & - & $\mathrm{X}$ & - \\
\hline Marssonella oxycona & - & - & - & - & - & - & - & - & - & - & $X$ & $X$ & $X$ & - & - \\
\hline Nodosaria affinis & - & $\mathrm{X}$ & $\mathrm{X}$ & $X$ & - & - & $X$ & - & - & - & - & - & - & - & - \\
\hline Nodosaria deliciae & - & - & $X$ & $X$ & - & - & $X$ & - & - & - & - & - & - & $X$ & - \\
\hline Nodosaria latejugata & - & $X$ & - & - & $X$ & - & - & - & - & - & - & - & - & $X$ & - \\
\hline Nodosaria limbata & - & - & - & - & - & - & - & - & - & - & $X$ & - & $\mathrm{X}$ & - & - \\
\hline Nodosaria longiscata & - & $X$ & $\mathrm{X}$ & $X$ & - & - & $X$ & $X$ & - & - & $X$ & $X$ & $\mathrm{X}$ & $\mathrm{X}$ & - \\
\hline Nodosaria macneili & - & - & $X$ & - & - & - & $X$ & - & - & - & $X$ & - & - & - & - \\
\hline Nodosaria spp. & - & - & - & - & - & - & - & $X$ & - & - & $X$ & - & $\mathrm{X}$ & $X$ & - \\
\hline Nonionella jacksonensis & - & - & - & - & - & - & - & - & - & - & - & - & - & $X$ & - \\
\hline Oridorsalis umbonatus & - & - & $X$ & - & - & - & - & - & - & - & - & - & - & - & - \\
\hline Osangularia mexicana & - & - & - & - & - & - & - & - & - & - & - & - & - & - & - \\
\hline Osangularia midwayana & - & $X$ & $X$ & - & - & - & $X$ & - & - & - & - & - & - & $X$ & $X$ \\
\hline Palmula primitiva & - & - & - & - & $\mathrm{X}$ & - & $X$ & - & - & - & - & - & - & - & - \\
\hline Planulina spp. & - & $X$ & - & - & $X$ & - & - & - & - & - & - & - & - & - & - \\
\hline Planulina truncana & - & $X$ & - & - & - & - & - & - & - & - & - & - & - & $X$ & - \\
\hline Plectina garzaensis & - & - & - & - & - & - & - & - & - & - & - & - & - & $X$ & - \\
\hline Praeglobobulimina ovata & - & - & - & - & - & - & - & - & - & - & - & - & - & $X$ & - \\
\hline Pseudoglandulina manifesta & - & $X$ & $X$ & $X$ & $X$ & - & $X$ & $X$ & - & - & - & - & - & - & - \\
\hline Quinqueloculina triangularis & - & - & - & - & $X$ & - & $X$ & - & - & - & - & - & - & - & - \\
\hline Reophax spp. & - & - & $X$ & - & - & - & $X$ & $X$ & $\mathrm{X}$ & - & - & - & - & - & - \\
\hline Rhabdammina eocenica & - & - & - & - & - & - & $X$ & - & - & - & - & - & - & - & - \\
\hline Saracenaria spp. & - & - & $X$ & $X$ & - & - & - & - & - & - & - & - & - & - & - \\
\hline Saracenaria triangularis & - & cf. & - & - & - & - & cf. & - & - & - & - & - & - & - & - \\
\hline
\end{tabular}




\begin{tabular}{|c|c|c|c|c|c|c|c|c|c|c|c|c|c|c|c|}
\hline \multirow[b]{2}{*}{$\begin{array}{l}\text { Benthic foraminifers from the } \\
\text { Vine Hill Sandstone in Block } \\
\text { IV }\end{array}$} & \multicolumn{3}{|c|}{ Selby Point } & \multicolumn{5}{|c|}{ Pacheco syncline } & \multicolumn{7}{|c|}{ Walnut Creek } \\
\hline & $\stackrel{\substack{N \\
N}}{N}$ & 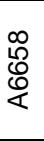 & 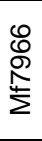 & $\frac{1}{N}$ & $\begin{array}{l}\overline{0} \\
\text { ê }\end{array}$ & 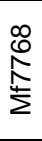 & 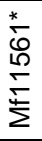 & $\frac{\substack{0 \\
N}}{\sum}$ & $\begin{array}{l}\infty \\
N \\
\infty \\
N \\
\sum\end{array}$ & 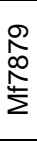 & $\begin{array}{l}0 \\
\frac{1}{\infty} \\
\frac{N}{\Sigma} \\
\Sigma\end{array}$ & $\begin{array}{l}\frac{1}{N} \\
\infty \\
\stackrel{N}{\Sigma}\end{array}$ & 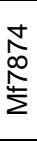 & 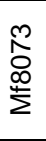 & $\begin{array}{l}\text { N } \\
\text { O } \\
\infty \\
\sum^{ \pm}\end{array}$ \\
\hline Silicosigmoilina californica & - & $\mathrm{X}$ & $\mathrm{X}$ & - & - & $\mathrm{X}$ & $\mathrm{X}$ & - & $\mathrm{X}$ & $\mathrm{X}$ & $\mathrm{X}$ & $\mathrm{X}$ & $\mathrm{X}$ & - & - \\
\hline Siphonia wilcoxensis & - & $X$ & - & - & - & - & - & - & - & - & - & - & - & $X$ & - \\
\hline Spiroplectammina directa & - & - & - & - & - & - & - & - & - & - & $X$ & $X$ & - & - & - \\
\hline Spiroplectammina richardi & - & $X$ & $X$ & $X$ & $X$ & - & $X$ & - & - & - & $X$ & - & $X$ & $X$ & $X$ \\
\hline Stensioina beccariformis & - & - & - & - & - & - & - & - & - & - & $X$ & - & $X$ & - & - \\
\hline Stilostomella adolphina & - & $X$ & $X$ & - & $X$ & - & $X$ & - & - & - & - & - & - & - & - \\
\hline Stilostomella paleocenica & - & - & - & - & - & - & $X$ & - & - & - & $X$ & - & $X$ & - & - \\
\hline Stilostomella spp. & - & - & $X$ & - & - & - & - & - & - & - & $X$ & - & - & $X$ & - \\
\hline Trifarina advena californica & - & - & - & - & - & - & - & - & - & - & - & - & - & $X$ & - \\
\hline Trifarina wilcoxensis & - & - & - & - & - & - & - & - & - & - & - & - & - & - & - \\
\hline Tritaxilina colei & - & - & - & - & - & - & - & - & - & $X$ & - & - & - & - & - \\
\hline Trochammina globigeriniformis & - & - & - & - & - & - & - & - & $X$ & $X$ & - & - & - & - & - \\
\hline Trochammina spp. & - & - & $X$ & $X$ & - & - & - & - & - & - & - & - & - & $X$ & - \\
\hline Uvigerina laimingi & - & $X$ & $X$ & $X$ & - & - & $X$ & - & - & - & - & - & - & - & - \\
\hline Uvigerina lodoensis miriamae & - & - & - & - & - & - & - & - & - & - & - & - & - & $X$ & - \\
\hline Vaginulinopsis asperuliformis & - & - & - & - & - & - & - & - & - & - & - & - & - & $X$ & X \\
\hline Vaginulinopsis earlandi & - & - & - & $X$ & - & - & $X$ & - & - & - & - & - & - & - & - \\
\hline Vaginulinopsis saundersi & - & - & - & - & $X$ & - & - & - & - & - & - & - & - & $X$ & - \\
\hline Vaginulinopsis tuberculata & - & $X$ & $X$ & $X$ & $X$ & - & $X$ & - & - & - & - & - & - & - & - \\
\hline Verneuilina triangulata & - & - & - & - & - & - & - & - & - & - & - & - & - & $\mathrm{X}$ & - \\
\hline
\end{tabular}

*Laboratory sample numbers Mf8265 and Mf11561 are assigned to the same sample.

In all three areas, the Vine Hill Sandstone was deposited at middle bathyal depths (500-2,000 m). This interpretation is based on species with upper depth limits in the middle bathyal biofacies including Bulimina macilenta, Gyroidina soldanii, Osangularia midwayensis, Silicosigmoilina californiensis, and various Stilostomella. The presence of Stensioina beccariformis in samples near Walnut Creek and few to no specimens of Nuttaloides truempyi suggests that deposition occurred at bathyal depths of 1,000-2,000 m (Katz and Miller, 1991). The Vine Hill Sandstone assemblages also contain considerable material transported from the outer shelf and upper slope.

The Las Juntas Shale unconformably overlies the Vine Hill Sandstone in this block. Graymer and others (1994a) considered this unit as Paleocene and (or) Eocene based on the work of Weaver (1953). However, as described below, only Eocene foraminifers are found in this unit. In the Selby Point section the two new samples (Mf7969 and Mf7970) from the Las Juntas Shale contain few age diagnostic species and are similar to the benthic foraminiferal assemblages found by Smith (1957) from the same section (A6659 and A6660)(table 18). The early Eocene age is based on the presence of longranging Eocene and younger species, and the abrupt disappearance of Paleocene species that are so common in the underlying Vine Hill Sandstone. 


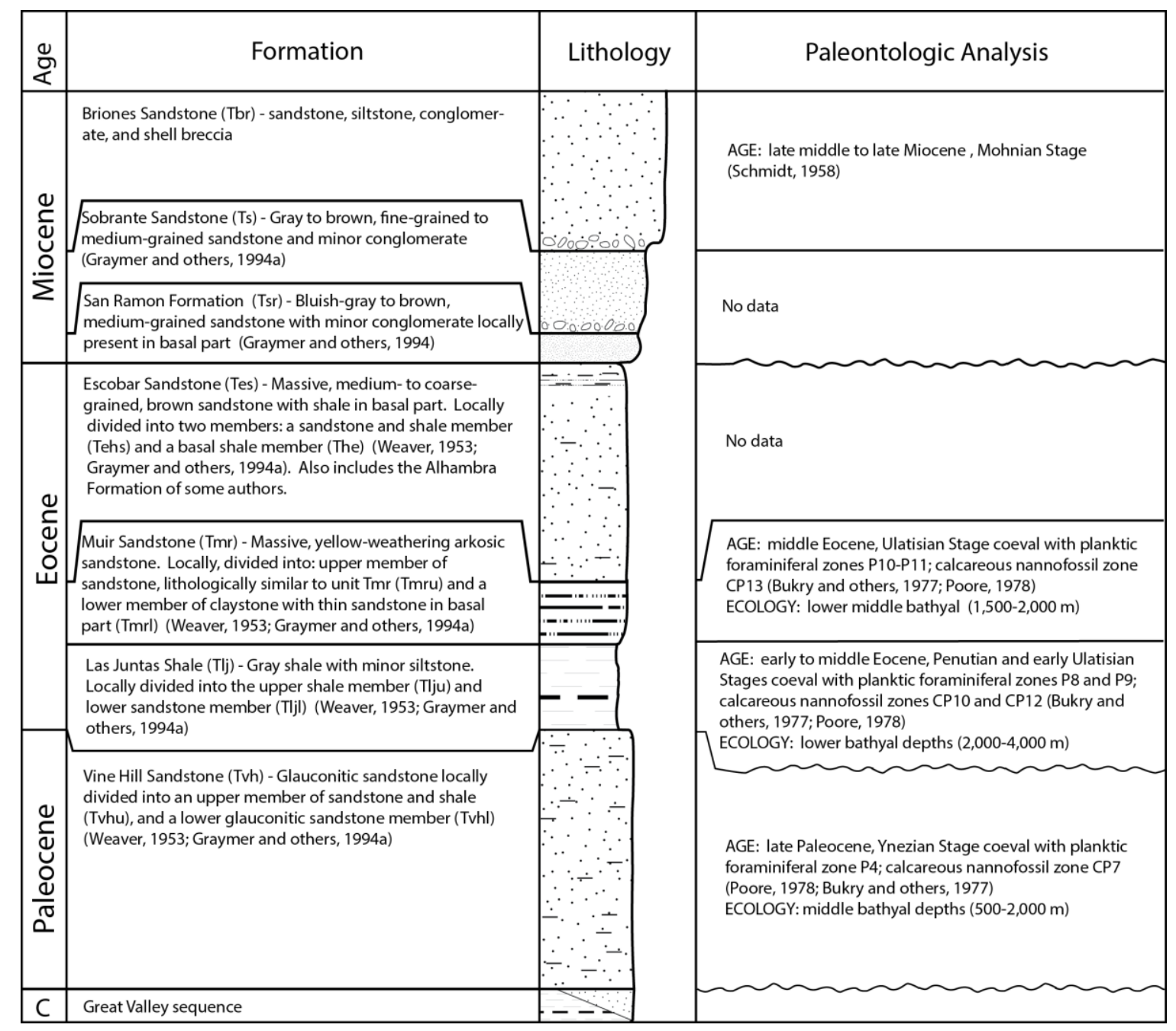

Figure 12. Composite columnar section for Block IV, Pacheco syncline. Age and environmental interpretations are summarized from the text. C, Cretaceous.

In the Santa Fe Railroad section, on the east limb of the Pacheco syncline, the Las Juntas Shale contains a diverse benthic foraminiferal assemblage that is early Eocene in age (table 18). This age is based on the first appearance of age diagnostic species such as Cibicidoides coalingensis, $C$. subspiratus, Plectofrondicularia paucicostata, and Pullenia eocenica; and last appearances of Allomorphina conica, Clavulina anglica, Gonatosphaera eocenica, Lituotuba lituiformis, Silicosigmoilina californica, Spiroplectammina directa, Trifarina wilcoxensis, and Vaginulinopsis saundersi. These species restrict the age to the early Eocene Penutian Stage, coeval with planktic foraminiferal zones P8 and P9. Calcareous nannofossils in sample Mf11560 near the base and Mf7770 near the top of the Las Juntas Shale in the Santa Fe Railroad section are assigned to zone CP10 (Bukry and others, 1977). Samples from the Las Juntas Shale exposed in the west limb of the Pacheco syncline, samples A6666 and A6665 (Smith, 1957), include many of the same age diagnostic species. The presence of Eponides mexicanus, Siphonia wilcoxensis, Vaginulinopsis nudicostata, and V. vacavillensis favors an early middle Eocene age, probably near the early/middle Eocene boundary. Calcareous nannofossils in samples A6666 and A6665 are assigned to the Discoaster sublodoensis zone (CP12) by Poore (1976) and also support an early middle Eocene age. 
Table 18. Benthic foraminifers from the Las Juntas shale in Block IV.

[Taxonomy of species is given in the section entitled "Taxonomic Notes.". X indicates the species is present; cf. indicates that the specimens resemble or compare to the species; aff. Indicates that the specimmens are related to but not identical with the species. Samples that contain few to rare diagnostic species include Mf7779 and Mf7780]

\begin{tabular}{|c|c|c|c|c|c|c|c|c|c|c|c|c|c|c|c|c|}
\hline \multirow[b]{2}{*}{$\begin{array}{l}\text { Benthic foraminifers from the } \\
\text { Las Juntas Shale in Block IV }\end{array}$} & \multicolumn{4}{|c|}{ Shelby Point } & \multicolumn{4}{|c|}{ Pacheco syncline } & \multicolumn{8}{|c|}{ Walnut Creek } \\
\hline & $\begin{array}{l}\text { 乃 } \\
\mathbb{0} \\
0 \\
\mathbb{4}\end{array}$ & 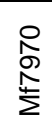 & $\begin{array}{l}8 \\
8 \\
8 \\
\end{array}$ & 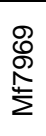 & 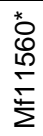 & $\begin{array}{l}\widetilde{\mho} \\
\mathbb{Z} \\
\mathbb{Z}\end{array}$ & 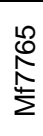 & $\begin{array}{l}\stackrel{R}{N} \\
\hat{N} \\
\stackrel{N}{\Sigma}\end{array}$ & 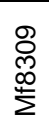 & $\begin{array}{l}\stackrel{0}{\bar{D}} \\
\dot{\infty} \\
\dot{\infty}\end{array}$ & $\begin{array}{l}\frac{\sigma}{\infty} \\
\stackrel{\infty}{\Sigma} \\
\dot{\infty}\end{array}$ & $\begin{array}{l}\stackrel{N}{D} \\
\stackrel{\infty}{\infty} \\
\sum\end{array}$ & $\begin{array}{l}\frac{m}{\infty} \\
\stackrel{\infty}{\infty} \\
\sum^{\prime}\end{array}$ & $\begin{array}{l}\underset{+}{\infty} \\
\stackrel{\infty}{\infty} \\
\dot{\infty}\end{array}$ & $\begin{array}{l}\frac{10}{\infty} \\
\frac{\infty}{\Sigma} \\
\sum\end{array}$ & $\begin{array}{l}\frac{0}{\infty} \\
\infty \\
\infty \\
\Sigma\end{array}$ \\
\hline Alabamina wilcoxensis & - & - & - & - & - & $x$ & - & - & $x$ & - & - & - & - & - & $x$ & - \\
\hline Allomorphina conica & - & - & - & - & - & - & - & $\mathrm{X}$ & $x$ & - & - & - & - & - & - & - \\
\hline Ammobacculites expansus & $x$ & - & $x$ & - & - & - & - & - & - & - & - & - & - & - & - & - \\
\hline Ammodiscus incertus & - & - & - & $x$ & - & - & - & $x$ & - & - & - & - & $x$ & $x$ & - & - \\
\hline Ammodiscus pennyi & - & $x$ & - & - & - & - & - & $\mathrm{X}$ & - & - & - & - & - & - & - & - \\
\hline Ammodiscus spp. & - & - & $x$ & $x$ & - & - & $x$ & - & - & - & - & - & - & - & - & - \\
\hline Amphimorphina becki & - & - & $x$ & - & - & - & - & - & - & - & - & - & - & - & - & - \\
\hline Amphimorphina ignota & - & - & - & - & $x$ & - & - & $\mathrm{X}$ & $x$ & - & - & - & - & $\mathrm{X}$ & - & - \\
\hline Anomalinoides acutus & - & - & - & - & - & $\mathrm{X}$ & - & - & - & - & - & - & - & - & - & - \\
\hline Anomalinoides capitatus & - & - & - & - & $x$ & $\mathrm{X}$ & - & $\mathrm{X}$ & - & - & $x$ & - & - & - & - & - \\
\hline Anomalinoides semicribratus & - & - & - & - & $x$ & - & - & - & - & - & - & - & - & - & - & - \\
\hline Anomalinoides spp. & - & - & - & - & - & - & - & - & $x$ & - & - & - & - & - & - & - \\
\hline Anomalina keenae & - & - & - & - & $x$ & - & - & $\mathrm{X}$ & $x$ & $\mathrm{X}$ & - & $x$ & - & $\mathrm{X}$ & $x$ & $\mathrm{X}$ \\
\hline Anomalina regina & - & - & - & - & - & $\mathrm{X}$ & - & - & $x$ & $\mathrm{X}$ & $x$ & $\mathrm{X}$ & - & $\mathrm{X}$ & - & $\mathrm{X}$ \\
\hline Aragonia aragonensis & - & - & - & - & - & - & - & - & $x$ & - & $x$ & - & - & - & - & - \\
\hline Astigerina simiensis & - & - & - & - & - & - & - & - & - & $X$ & - & - & - & - & - & - \\
\hline Bathysiphon eocenicus & - & - & - & $x$ & - & - & - & - & - & - & - & $X$ & - & $x$ & $x$ & - \\
\hline Bathysiphon santecruis & $x$ & $x$ & $x$ & $x$ & $x$ & $\mathrm{X}$ & $x$ & $\mathrm{X}$ & - & - & - & - & - & - & - & - \\
\hline Bathysiphon spp. & $x$ & $x$ & $x$ & $x$ & - & - & $x$ & - & - & - & - & - & - & $x$ & - & $x$ \\
\hline Buliminella grata & - & - & - & - & - & - & - & - & - & - & - & - & - & $x$ & $x$ & - \\
\hline Buliminella robertsi & - & - & - & - & $x$ & $x$ & - & $x$ & - & - & - & - & - & - & - & - \\
\hline Bolivina explicata lodoensis & - & - & - & - & - & - & - & - & $x$ & $x$ & $x$ & - & - & - & - & - \\
\hline Bolivinoides delicatulus & - & - & - & - & - & $x$ & - & - & - & - & - & - & - & - & - & - \\
\hline Bulimina alazaensis & - & - & - & - & $x$ & - & - & $x$ & $x$ & $\mathrm{X}$ & $x$ & - & $x$ & $x$ & - & - \\
\hline Bulimina bradburyi & - & - & - & - & - & - & - & $x$ & - & - & - & - & - & - & - & - \\
\hline Bulimina callahani & - & - & - & - & $x$ & - & - & $x$ & $x$ & $x$ & $x$ & - & - & - & - & - \\
\hline Bulimina macilenta & - & - & - & - & $x$ & $x$ & - & $x$ & $x$ & - & $x$ & - & - & $x$ & $x$ & - \\
\hline Bulimina semicostata & - & - & - & - & - & - & - & - & $x$ & - & $x$ & - & - & - & - & - \\
\hline Bulimina trinitatensis & - & - & - & - & $x$ & - & - & $x$ & $x$ & - & $x$ & - & - & - & - & - \\
\hline Bulimina tuxapamensis & - & - & - & - & $x$ & - & - & - & - & - & - & - & - & - & - & - \\
\hline Chrysalongonium elongatum & - & - & - & - & - & - & - & $x$ & $x$ & - & - & - & - & $x$ & - & - \\
\hline Chrysalongonium spp. & - & - & - & - & $x$ & - & - & - & - & - & - & $x$ & - & - & - & - \\
\hline Chrysalongonium teniucostatum & - & - & - & - & - & - & - & $\mathrm{X}$ & - & - & - & - & - & - & - & - \\
\hline Cibicides beatus & - & - & - & - & - & - & - & $x$ & - & - & - & - & - & - & - & - \\
\hline Cibicides kernensis & - & - & - & - & - & - & - & - & $x$ & cf. & - & - & - & - & - & - \\
\hline Cibicides kleinpelli & - & - & - & - & - & $x$ & - & - & - & - & - & - & - & - & - & - \\
\hline Cibicides madrugaensis & - & - & - & - & - & $\mathrm{X}$ & - & - & - & - & - & - & - & - & - & - \\
\hline Cibicidoides coalingensis & - & - & - & - & - & $\mathrm{X}$ & - & - & - & - & - & - & - & - & - & - \\
\hline Cibicidoides eocaenus & - & - & - & - & $x$ & - & - & $\mathrm{X}$ & $x$ & $x$ & - & - & - & $\mathrm{X}$ & $x$ & $\mathrm{X}$ \\
\hline Cibicidoides eponidiformis & - & - & - & - & - & $x$ & - & $x$ & - & - & - & - & - & - & - & - \\
\hline Cibicidoides praemundulus & - & - & - & - & $\mathrm{X}$ & - & - & - & $x$ & $\mathrm{X}$ & $x$ & - & - & - & - & - \\
\hline Cibicidoides subspiratus & - & - & - & - & - & - & - & $x$ & $x$ & $x$ & $x$ & $x$ & $x$ & $X$ & $x$ & - \\
\hline Cibicidoides spp. & - & - & - & - & $\mathrm{X}$ & - & - & $x$ & $x$ & $\mathrm{X}$ & - & - & - & $X$ & - & - \\
\hline Cibicides pseudoungerianus & - & - & - & - & $x$ & $x$ & - & - & - & - & - & - & - & - & - & - \\
\hline Clavulina anglica & - & $x$ & - & - & - & - & - & $\mathrm{X}$ & - & - & - & $\mathrm{X}$ & - & $\mathrm{X}$ & $x$ & $\mathrm{X}$ \\
\hline Cribrostomoides spp. & $x$ & - & $x$ & - & - & - & - & - & - & - & - & - & - & - & - & - \\
\hline
\end{tabular}




\begin{tabular}{|c|c|c|c|c|c|c|c|c|c|c|c|c|c|c|c|c|}
\hline \multirow[b]{2}{*}{$\begin{array}{l}\text { Benthic foraminifers from the } \\
\text { Las Juntas Shale in Block IV }\end{array}$} & \multicolumn{4}{|c|}{ Shelby Point } & \multicolumn{4}{|c|}{ Pacheco syncline } & \multicolumn{8}{|c|}{ Walnut Creek } \\
\hline & $\begin{array}{l}\stackrel{0}{0} \\
0 \\
\mathbb{0}\end{array}$ & 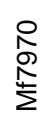 & $\begin{array}{l}8 \\
8 \\
\mathbb{8} \\
\mathbb{1}\end{array}$ & $\begin{array}{l}8 \\
\mathscr{8} \\
\stackrel{2}{2} \\
\sum\end{array}$ & $\begin{array}{l}{ }^{*} \\
0 \\
10 \\
\Sigma \\
\Sigma \\
\Sigma\end{array}$ & 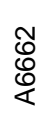 & 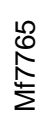 & $\begin{array}{l}\stackrel{P}{N} \\
\stackrel{N}{\Sigma}\end{array}$ & 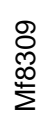 & $\begin{array}{l}0 \\
\frac{0}{\infty} \\
\infty \\
\stackrel{\infty}{L}\end{array}$ & $\begin{array}{l}\frac{\tau}{\infty} \\
\stackrel{\infty}{\infty} \\
\sum\end{array}$ & 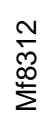 & $\begin{array}{l}\frac{m}{m} \\
\frac{0}{\infty} \\
\frac{\infty}{\Sigma}\end{array}$ & $\begin{array}{l}\frac{\nabla}{M} \\
\stackrel{\infty}{\Sigma} \\
\dot{\infty}\end{array}$ & $\begin{array}{l}\frac{10}{M} \\
\frac{0}{2} \\
\sum\end{array}$ & $\begin{array}{l}\frac{0}{\infty} \\
\infty \\
\stackrel{\infty}{\Sigma}\end{array}$ \\
\hline Cyclammina clarki & $x$ & - & $x$ & - & - & - & - & - & - & - & - & - & - & - & - & - \\
\hline Cyclammina pacifica & - & - & - & - & - & - & - & - & $x$ & - & - & $x$ & - & - & - & - \\
\hline Cyclammina samanica & - & $x$ & - & - & - & - & - & - & - & - & - & - & - & $x$ & $x$ & - \\
\hline Cyclammina simiensis & - & - & - & - & - & - & $x$ & $X$ & - & - & - & - & - & - & - & - \\
\hline Cyclammina aff. C. simiensis & - & - & - & - & - & - & $x$ & - & - & - & - & - & - & - & - & - \\
\hline Cyclammina spp. & - & - & - & $\mathrm{X}$ & - & - & $x$ & - & - & - & - & - & - & - & - & - \\
\hline Dentalina colei & - & - & - & - & - & - & - & - & $x$ & $x$ & - & - & - & - & - & - \\
\hline Dentalina communis & - & - & - & - & - & - & - & $x$ & $x$ & $x$ & - & $\mathrm{X}$ & - & $\mathrm{X}$ & - & - \\
\hline Dentalina consobrina & - & - & - & - & $x$ & $x$ & - & $x$ & - & - & - & $x$ & - & - & - & - \\
\hline Dentalina globulicauda & - & - & - & - & - & - & - & $\mathrm{X}$ & - & - & - & - & - & - & - & - \\
\hline Dentalina pseudobliquestriata & - & - & - & - & $\mathrm{X}$ & - & - & - & - & - & - & - & - & - & - & - \\
\hline Discorbis baintoni & - & - & - & - & - & - & - & - & $x$ & $x$ & - & - & - & - & - & - \\
\hline Discorbis spp. & - & - & - & - & - & - & - & - & - & $x$ & - & - & - & - & - & - \\
\hline Dorothia bulletta & - & - & - & $x$ & - & - & $x$ & $x$ & - & - & - & - & - & - & - & - \\
\hline Dorothia principiensis & - & - & - & - & - & - & - & $\mathrm{X}$ & - & - & - & - & - & - & $\mathrm{X}$ & - \\
\hline Dorothia spp. & - & - & - & - & $\mathrm{x}$ & - & - & - & - & - & - & - & - & - & - & - \\
\hline Ellipsoglandulina multicostata & - & - & - & - & $x$ & - & - & - & - & - & - & - & - & - & - & - \\
\hline Epistominella spp. & - & - & - & - & - & - & - & - & - & $x$ & - & - & - & - & - & - \\
\hline Eponides lodoensis & - & - & - & - & $\mathrm{x}$ & - & - & $x$ & $x$ & - & - & - & $x$ & - & $x$ & $x$ \\
\hline Eponides mexicanus & - & - & - & - & aff. & $x$ & - & - & - & - & - & - & - & - & - & - \\
\hline Fissurina alveolata & - & - & - & - & - & - & - & cf. & - & - & - & - & - & - & - & - \\
\hline Gaudryina laevigata & - & - & - & - & - & - & - & $x$ & - & - & - & - & - & - & - & - \\
\hline Gaudryina spp. & - & - & - & - & - & - & $x$ & - & - & - & - & - & - & - & - & - \\
\hline Globocassidulina globosa & - & - & - & - & - & $x$ & - & $x$ & - & $x$ & - & - & $x$ & - & $x$ & $x$ \\
\hline Glandulina laevigata & - & - & - & - & - & - & - & $x$ & - & $x$ & - & - & - & $x$ & - & - \\
\hline Globulina spp. & - & - & - & - & - & - & - & - & $x$ & - & - & - & - & - & - & - \\
\hline Glomospira charoides & - & - & - & - & - & - & - & $x$ & - & - & - & - & $x$ & - & - & - \\
\hline Gonatosphaera eocenica & - & - & - & - & $x$ & - & - & - & - & - & - & - & - & - & - & - \\
\hline Gyroidina condoni & - & - & - & - & - & - & - & $x$ & - & - & - & - & - & - & - & - \\
\hline Gyroidina octocameratus & - & - & - & - & - & $x$ & - & - & - & - & - & - & $x$ & - & - & - \\
\hline Gyroidina soldanii & - & - & - & - & - & - & - & - & $x$ & - & - & - & $x$ & $x$ & $x$ & - \\
\hline Gyroidina spp. & - & - & - & - & - & - & - & - & $x$ & - & $x$ & - & - & - & - & - \\
\hline Hanzawaia blanpiedi & - & - & - & - & - & - & - & - & $x$ & - & - & - & - & - & - & - \\
\hline Hanzawaia mauricensis & - & - & - & - & - & - & - & - & $x$ & $x$ & $x$ & - & - & - & - & $x$ \\
\hline Hanzawaia spp. & - & - & - & - & - & - & - & - & - & - & - & - & - & - & - & $\mathrm{X}$ \\
\hline Haplophragmoides eggeri & - & $x$ & - & $\mathrm{X}$ & - & - & $x$ & $x$ & - & - & - & - & $x$ & $x$ & - & $\mathrm{x}$ \\
\hline Haplophragmoides excavata & - & $x$ & - & - & - & - & - & - & - & - & - & - & - & - & - & - \\
\hline Haplophragmoides glabra & $x$ & - & - & - & - & - & - & - & - & - & - & - & - & - & - & - \\
\hline Haplophragmoides spp. & - & - & - & - & - & - & $x$ & $x$ & - & - & - & - & - & $x$ & - & - \\
\hline Hoeglundina eocenica & - & - & - & - & - & - & - & - & - & - & - & $x$ & $x$ & $\mathrm{X}$ & - & - \\
\hline Hormosina spp. & - & - & - & - & - & - & - & $x$ & - & - & - & - & - & - & - & - \\
\hline Karreriella conversa & - & - & - & - & - & - & - & - & - & - & - & - & - & $x$ & - & - \\
\hline Karreriella horrida & - & - & - & - & - & - & - & - & - & - & - & - & - & - & $x$ & - \\
\hline Karreriella spp. & - & - & - & - & - & - & - & - & $x$ & - & - & - & $x$ & - & - & - \\
\hline Lagena costata & - & - & - & - & - & $x$ & - & - & $x$ & $x$ & $x$ & - & - & - & - & - \\
\hline Lagena gracilis & - & - & - & - & $\mathrm{x}$ & - & - & - & - & - & - & - & - & - & - & - \\
\hline Lagena paucicosta & - & - & - & - & - & $x$ & - & - & - & - & - & - & - & - & - & - \\
\hline Lagena spp. & - & - & - & - & - & - & - & - & $x$ & - & - & - & - & - & - & - \\
\hline Lagena striata & - & - & - & - & - & - & - & - & $x$ & - & - & - & - & - & - & - \\
\hline Lagena vulgaris & - & - & - & - & - & - & - & $X$ & - & - & - & - & - & - & - & - \\
\hline Lenticulina altolimbatus & - & - & - & - & $x$ & - & - & $x$ & - & - & - & $x$ & - & - & - & - \\
\hline Lenticulina arcuatostriata & - & - & - & - & - & - & - & - & - & - & - & $\mathrm{X}$ & $\mathrm{X}$ & - & - & - \\
\hline
\end{tabular}




\begin{tabular}{|c|c|c|c|c|c|c|c|c|c|c|c|c|c|c|c|c|}
\hline \multirow[b]{2}{*}{$\begin{array}{l}\text { Benthic foraminifers from the } \\
\text { Las Juntas Shale in Block IV }\end{array}$} & \multicolumn{4}{|c|}{ Shelby Point } & \multicolumn{4}{|c|}{ Pacheco syncline } & \multicolumn{8}{|c|}{ Walnut Creek } \\
\hline & $\begin{array}{l}\text { 怘 } \\
0 \\
0 \\
\mathbb{0}\end{array}$ & 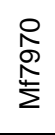 & $\begin{array}{l}8 \\
8 \\
8 \\
8\end{array}$ & $\begin{array}{l}\text { ஜ } \\
\mathbb{8} \\
\stackrel{N}{\Sigma}\end{array}$ & 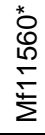 & $\begin{array}{l}\widetilde{Z} \\
\mathbb{E} \\
\mathbb{Z}\end{array}$ & $\begin{array}{l}0 \\
\stackrel{1}{0} \\
\stackrel{N}{\Sigma} \\
\sum\end{array}$ & $\begin{array}{l}\stackrel{0}{N} \\
\stackrel{N}{\Sigma}\end{array}$ & $\begin{array}{l}\text { ஜ } \\
\text { ஜ } \\
\stackrel{0}{\Sigma} \\
\sum\end{array}$ & $\begin{array}{l}\frac{0}{m} \\
\infty \\
\dot{\infty}\end{array}$ & $\begin{array}{l}\frac{\sigma}{\infty} \\
\frac{0}{\Sigma} \\
\sum\end{array}$ & $\begin{array}{l}\stackrel{N}{N} \\
\frac{\infty}{\Sigma} \\
\sum\end{array}$ & $\begin{array}{l}\frac{m}{m} \\
\frac{\infty}{\Sigma} \\
\sum\end{array}$ & $\begin{array}{l}\underset{⿱ 亠 乂}{\infty} \\
\underset{\infty}{\Sigma}\end{array}$ & $\begin{array}{l}\frac{10}{\infty} \\
\frac{\infty}{\Sigma} \\
\sum\end{array}$ & $\begin{array}{l}\frac{0}{m} \\
\frac{\infty}{\Sigma} \\
\sum\end{array}$ \\
\hline Lenticulina inornata & - & - & - & - & - & $x$ & - & - & - & - & - & - & - & - & - & - \\
\hline Lenticulina limbosa & - & - & - & - & - & - & - & - & - & - & - & - & - & - & - & $\mathrm{X}$ \\
\hline Lenticulina limbosa hockleyensis & - & - & - & - & - & - & - & $\mathrm{X}$ & - & - & - & - & - & - & - & - \\
\hline Lenticulina pseudocultratus & - & - & - & - & - & - & - & $\mathrm{X}$ & - & - & - & $x$ & $x$ & - & - & $\mathrm{X}$ \\
\hline Lenticulina pseudovortex & - & - & - & - & $x$ & - & - & - & - & - & - & $x$ & $x$ & - & - & $\mathrm{X}$ \\
\hline Lenticulina spp. & - & - & - & - & - & $\mathrm{X}$ & - & - & $x$ & $\mathrm{X}$ & $x$ & $x$ & $x$ & $X$ & $x$ & $\mathrm{X}$ \\
\hline Lenticulina terryi & - & - & - & - & $x$ & - & - & - & - & - & - & - & - & - & - & - \\
\hline Lenticulina turbinatus & - & - & - & - & - & - & - & $X$ & - & - & - & - & - & - & - & - \\
\hline Lituotuba lituiformis & - & - & - & - & - & - & - & $X$ & - & - & - & - & - & - & - & - \\
\hline Loxostomoides applinae & - & - & - & - & - & - & - & - & - & - & $x$ & - & - & - & - & - \\
\hline Marginulina exima & - & - & - & - & - & - & - & - & $x$ & - & - & - & - & - & - & - \\
\hline Marginulina spp. & - & - & - & - & - & - & - & - & $x$ & - & - & - & - & - & - & - \\
\hline Nonionella spp. & - & - & - & - & - & - & - & - & $x$ & $\mathrm{X}$ & $x$ & - & - & - & - & - \\
\hline Nodosaria deliciae & - & - & - & - & - & $x$ & - & $\mathrm{X}$ & $x$ & - & - & - & $x$ & $X$ & - & - \\
\hline Nodosaria gyrata & - & - & - & - & - & - & - & - & - & - & - & - & - & $X$ & - & - \\
\hline Nodosaria latejugata & - & - & - & - & - & $x$ & - & $x$ & - & - & - & $x$ & $x$ & $x$ & - & $x$ \\
\hline Nodosaria longiscata & - & - & - & - & $x$ & $\mathrm{X}$ & - & $\mathrm{X}$ & $x$ & $X$ & $x$ & $x$ & $x$ & $X$ & $x$ & $\mathrm{X}$ \\
\hline Nodosaria spp. & - & - & - & - & - & - & - & $x$ & - & $x$ & $x$ & - & $x$ & $X$ & - & $\mathrm{X}$ \\
\hline Nodosaria velascoensis & - & - & - & - & $x$ & - & - & - & - & - & - & - & - & - & - & - \\
\hline Nonion inexcavatum & - & - & - & - & - & $\mathrm{X}$ & - & - & - & - & - & - & - & - & - & - \\
\hline Nuttaloides truempyi & - & - & - & - & $x$ & - & - & $\mathrm{X}$ & $x$ & $\mathrm{X}$ & $x$ & - & - & $X$ & - & $X$ \\
\hline Oridorsalis spp. & - & - & - & - & - & - & - & $x$ & - & - & - & - & - & - & - & - \\
\hline Oridorsalis umbonatus & - & - & - & - & $x$ & - & - & $X$ & - & - & $x$ & $X$ & $X$ & $X$ & - & - \\
\hline Osangularia mexicana & - & - & - & - & $x$ & - & - & $x$ & $x$ & - & - & - & - & $X$ & $x$ & $\mathrm{X}$ \\
\hline Osangularia midwayana & - & - & - & - & - & $x$ & - & - & - & - & - & - & - & - & - & - \\
\hline Planularia spp. & - & - & - & - & - & - & - & - & - & - & - & - & - & $\mathrm{X}$ & - & - \\
\hline Plectina garzaensis & - & - & - & - & - & - & - & cf. & - & - & - & - & - & - & - & - \\
\hline Plectofrondicularia paucicostata & - & - & - & - & $x$ & - & - & - & $x$ & - & - & - & - & - & - & - \\
\hline Pleurostomella spp. & - & - & - & - & - & - & - & - & - & - & - & - & - & $X$ & - & $\mathrm{X}$ \\
\hline Praeglobobulimina ovata & - & - & - & - & - & - & - & - & - & - & - & - & - & - & - & $\mathrm{X}$ \\
\hline Praeglobobulimina pupoides & - & - & - & - & - & - & - & - & $\mathrm{X}$ & $\mathrm{X}$ & - & - & $x$ & $\mathrm{X}$ & - & $\mathrm{X}$ \\
\hline Pullenia eocenica & - & - & - & - & $x$ & - & - & $\mathrm{X}$ & $\mathrm{x}$ & - & - & - & - & - & - & $\mathrm{X}$ \\
\hline Pullenia quatriloba & - & - & - & - & $x$ & - & - & - & - & - & - & - & - & - & - & - \\
\hline Pullenia quinqueloba & - & - & - & - & - & - & - & - & $x$ & - & - & - & - & - & - & - \\
\hline Pullenia salisburyi & - & - & - & - & $x$ & $\mathrm{X}$ & - & $\mathrm{X}$ & - & $\mathrm{X}$ & - & - & - & - & - & - \\
\hline Pyrgo spp. & - & - & - & - & - & - & - & - & - & $\mathrm{X}$ & - & - & - & - & - & - \\
\hline Quinqueloculina josephina & - & - & - & - & - & - & - & - & $\mathrm{x}$ & $\mathrm{X}$ & - & - & - & - & - & - \\
\hline Quinqueloculina spp. & - & - & - & - & - & - & - & - & - & $X$ & - & - & - & - & - & - \\
\hline Quinqueloculina triangularis & - & - & - & - & - & - & - & - & $\mathrm{X}$ & $X$ & - & - & - & - & - & $X$ \\
\hline Reophax spp. & - & - & - & - & - & - & $X$ & - & - & - & - & - & - & - & - & - \\
\hline Saccammina spp. & - & - & - & - & - & - & - & - & - & - & - & - & $x$ & - & - & - \\
\hline Sigmoilina spp. & - & - & - & - & - & $\mathrm{X}$ & - & - & - & - & - & - & - & - & - & - \\
\hline Sigmoilina tenuis & - & - & - & - & - & - & - & - & $x$ & - & - & - & - & $\mathrm{X}$ & - & - \\
\hline Silicosigmoilina californica & $x$ & $x$ & $\mathrm{X}$ & $\mathrm{X}$ & - & - & $x$ & $\mathrm{X}$ & $x$ & - & - & - & $x$ & $X$ & - & - \\
\hline Siphonodosaria gracillima & - & - & - & - & - & - & - & - & $x$ & $\mathrm{X}$ & $x$ & - & - & - & - & - \\
\hline Siphonia wilcoxensis & - & - & - & - & - & - & - & - & $x$ & $\mathrm{X}$ & $x$ & - & - & - & - & - \\
\hline Spiroplectammina directa & - & $x$ & $x$ & $x$ & - & $x$ & $x$ & $x$ & - & - & - & - & $x$ & - & - & $x$ \\
\hline Spiroplectammina richardi & $x$ & - & - & - & $x$ & - & $\mathrm{X}$ & - & $\mathrm{X}$ & $\mathrm{X}$ & - & - & - & $X$ & - & $\mathrm{X}$ \\
\hline Stilostomella adolphina & - & - & - & - & $x$ & $\mathrm{X}$ & - & - & - & - & - & - & - & - & - & - \\
\hline Stilostomella advena & - & - & - & - & - & - & - & - & - & - & - & $x$ & - & - & - & - \\
\hline Stilostomella lepidula & - & - & - & - & - & - & - & $x$ & $x$ & $x$ & - & $x$ & $x$ & $x$ & - & - \\
\hline Stilostomella spp. & - & - & - & - & - & $\mathrm{X}$ & - & - & - & - & - & - & - & - & - & - \\
\hline
\end{tabular}




\begin{tabular}{|c|c|c|c|c|c|c|c|c|c|c|c|c|c|c|c|c|}
\hline \multirow[b]{2}{*}{$\begin{array}{l}\text { Benthic foraminifers from the } \\
\text { Las Juntas Shale in Block IV }\end{array}$} & \multicolumn{4}{|c|}{ Shelby Point } & \multicolumn{4}{|c|}{ Pacheco syncline } & \multicolumn{8}{|c|}{ Walnut Creek } \\
\hline & 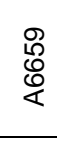 & $\begin{array}{l}\text { P } \\
\text { D } \\
\stackrel{F}{\Sigma}\end{array}$ & $\begin{array}{l}8 \\
\vdots \\
8\end{array}$ & $\begin{array}{l}\mathscr{8} \\
\mathscr{8} \\
\stackrel{D}{\Sigma} \\
\sum\end{array}$ & 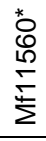 & 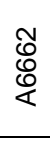 & $\begin{array}{l}\stackrel{L}{0} \\
\stackrel{0}{L} \\
\stackrel{\Sigma}{\Sigma}\end{array}$ & $\begin{array}{l}\stackrel{P}{N} \\
\frac{N}{\Sigma}\end{array}$ & 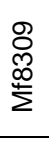 & $\begin{array}{l}\frac{0}{0} \\
\stackrel{\infty}{\Sigma} \\
\frac{\infty}{\Sigma}\end{array}$ & $\begin{array}{l}\bar{\Gamma} \\
\stackrel{\infty}{\Sigma}\end{array}$ & $\begin{array}{l}\frac{N}{\infty} \\
\stackrel{\infty}{\Sigma}\end{array}$ & $\begin{array}{l}\frac{M}{\infty} \\
\stackrel{\infty}{\infty} \\
\sum\end{array}$ & $\begin{array}{l}\stackrel{\nabla}{\infty} \\
\stackrel{\infty}{\Sigma}\end{array}$ & $\begin{array}{l}\frac{10}{\infty} \\
\stackrel{\infty}{\Sigma} \\
\Sigma\end{array}$ & $\begin{array}{l}\frac{\varphi}{\mathscr{D}} \\
\frac{\infty}{\Sigma}\end{array}$ \\
\hline Textularia adalta & - & - & - & - & - & cf. & $\mathrm{x}$ & - & & - & - & - & - & - & - & - \\
\hline Textularia spp. & - & $\mathrm{x}$ & - & $\mathrm{x}$ & - & - & - & - & $\mathrm{x}$ & $\mathrm{x}$ & - & - & - & $\mathrm{x}$ & - & - \\
\hline Trifarina advena californica & - & - & - & - & - & - & - & - & $x$ & - & - & - & - & - & - & $\mathrm{x}$ \\
\hline Trifarina wilcoxensis & - & - & - & - & - & $x$ & - & - & - & - & - & - & - & - & - & - \\
\hline Tritaxilina colei & - & $\mathrm{x}$ & - & - & - & - & $\mathrm{x}$ & - & $\mathrm{x}$ & $\mathrm{x}$ & - & - & $\mathrm{x}$ & $\mathrm{x}$ & - & - \\
\hline Trochammina globigeriniformis & cf. & $\mathrm{x}$ & cf. & $\mathrm{x}$ & - & - & $\mathrm{x}$ & $\mathrm{x}$ & 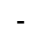 & - & - & - & - & - & - & - \\
\hline Trochammina spp. & $\mathrm{x}$ & $\mathrm{x}$ & - & - & $x$ & - & - & - & - & - & - & - & - & - & - & - \\
\hline Trochamminoides contortus & - & - & - & - & - & - & - & $\mathrm{x}$ & - & - & - & - & - & - & - & - \\
\hline Uvigerina churchi & - & - & - & - & - & - & - & - & - & $\mathrm{x}$ & - & - & - & - & - & - \\
\hline Uvigerina gardnerae & - & - & - & - & $\mathrm{x}$ & - & - & - & - & - & - & - & - & - & - & - \\
\hline Uvigerina hispida & - & - & - & - & - & - & - & - & $\mathrm{x}$ & - & $\mathrm{x}$ & - & - & - & - & - \\
\hline Uvigerina lodoensis miriamae & - & - & - & - & - & - & - & - & $\mathrm{x}$ & $\mathrm{x}$ & $\mathrm{x}$ & - & - & - & - & $\mathrm{x}$ \\
\hline Uvigerina spp. & - & - & - & - & - & $\mathrm{x}$ & - & - & - & - & - & - & - & - & - & - \\
\hline Vaginulinopsis asperuliformis & - & - & - & - & $\mathrm{x}$ & - & - & - & - & - & - & - & $\mathrm{x}$ & - & - & $\mathrm{x}$ \\
\hline Vaginulinopsis nudicostata & - & - & - & - & - & - & - & - & - & - & - & $x$ & - & - & - & - \\
\hline Vaginulinopsis saundersi & - & - & - & - & - & $\mathrm{x}$ & - & - & $x$ & - & $\mathrm{x}$ & - & $x$ & - & - & - \\
\hline Vaginulinopsis spp. & - & - & - & - & - & - & - & - & - & - & - & - & - & $\mathrm{X}$ & - & - \\
\hline Vaginulinopsis verruculosa & - & - & - & - & - & $\mathrm{x}$ & - & - & - & - & - & - & - & - & - & - \\
\hline Valvulineria martinezensis & - & - & - & - & 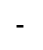 & $x$ & - & - & - & - & - & - & - & - & - & - \\
\hline Valvulineria spp. & - & - & - & - & - & - & - & - & - & - & - & - & - & - & - & $\mathrm{x}$ \\
\hline Verneuilina triangulata & - & - & - & - & - & - & - & - & $\mathrm{x}$ & $\mathrm{x}$ & - & $\mathrm{x}$ & - & - & $x$ & \\
\hline
\end{tabular}

* Laboratory sample numbers Mf8251, Mf11560, Mf10250 are assigned to the same sample.

Samples taken near Walnut Creek contain benthic foraminifers that suggest an early to middle Eocene age. The records for these samples show them located in strata mapped as San Ramon Sandstone and adjacent to the contact between the San Ramon Sandstone and Las Juntas Shale, but a slight westward adjustment of the location would place the samples in the Las Juntas Shale, which would be more compatible with the microfaunal interpretation. These assemblages are late early to early middle Eocene in age (table 18) and are assigned to the latest Penutian through earliest Ulatisian Stages, coeval with planktic foraminiferal zone P9. This age is based on the first appearances of Eponides mexicanus, Hanzawaia mauricensis, Lenticulina cf. L. welchi, Plectofrondicularia paucicostata, Uvigerina churchi, and Vaginulinopsis nudicostata; the last appearances of Anomalina regina, Bolivina explicata lodoensis, Bulimina callahani, Clavulina anglica, Karreriella conversa, Loxostomoides applinae, Spiroplectammina directa, Uvigerina lodoensis miriamae, and Vaginulinopsis saundersi; and the presence of Cibicidoides subspiratus and Pullenia eocenica. Although Eponides mexicanus and Siphonia wilcoxensis have longer ranges, they commonly occur in the late Penutian and early Ulatisian Stage interval in California during a period of lowered sea level in planktic foraminiferal zone P9.

Deposition of the Las Juntas Shale occurred at bathyal depths (500-2,000 m) in the Selby Point section and at lower bathyal depths (2,000-4,000 m) in the Pacheco syncline and Walnut Creek areas. The Selby Point section assemblages contain only arenaceous species, which have broader bathymetric ranges; therefore a more definitive bathymetric range is not possible. In the other areas, the bathymetric interpretation is based on the presence of species with upper depth limits in the lower bathyal biofacies such as Cibicidoides praemundulus, Glomospira charoides (rare to few), Gyroidina soldanii, Nuttaloides truempyi, and Pleurostomella spp. (rare). Common to abundant Nuttaloides truempyi indicates depths of greater than 2,500 m (Katz and Miller, 1991; van Morkhoven and others, 1986). The assemblages in the west limb of the Pacheco syncline (A6665 and A6666) contain only outer neritic and 
upper bathyal species, which are interpreted as transported from the shelf and therefore indicate a minimum depth only. Upper slope- and shelf-dwelling species are common to abundant throughout the west limb of the Pacheco syncline and indicate considerable erosion and downslope transport.

Four samples (Mf7771 through Mf7774) were taken from the Muir Sandstone in the east limb of the Pacheco syncline. Only one sample contains diagnostic faunas (table 19): sample Mf7773 contains a diverse assemblage that is middle Eocene and assigned to the Ulatisian Stage coeval with planktic foraminiferal zones P10 and P11. The middle Eocene age is based on the presence of Amphimorphina californica, Cibicidoides subspiratus, Eggerella elongata, Lenticulina ulatisensis, L. welchi, Silicosigmoilina californica, and Uvigerina elongata (table 17). The abundant calcareous nannoplankton in sample Mf7773 and in Smith's (1957) samples are assigned to the middle Eocene zone CP13 (Poore, 1976; Bukry and others, 1998) and thus agree with the benthic foraminiferal age interpretation. Benthic foraminiferal species indicate deposition occurred at lower middle bathyal depths $(1,500-2,000 \mathrm{~m})$.

The section of Muir Sandstone examined by Smith (1957) along the west limb of the Pacheco syncline (samples A6667 through A6687) contains a more diverse benthic foraminiferal assemblage and is also interpreted as middle Eocene in age. The faunal assemblage contains numerous species that first appear in the early Eocene and last appear in the middle or late Eocene. The only species that provide any age restrictions are Vaginulinopsis vacavillensis in A6685, V. verruculosa in A6683, and Uvigerina lodoensis mirmarae in A6686. These species last appear in the early Ulatisian Stage, coeval with planktic foraminiferal zones late P9 through early P10. This assemblage is, therefore, assigned to the middle Eocene Ulatisan Stage. The presence of Siphonia wilcoxensis in samples A6667, A6673, A6674, and A6675 is consistent with an early middle Eocene age. Calcareous nannofossils in this section also indicate a middle Eocene age and are assigned to zone CP13 (Poore, 1976).

No samples were examined from the overlying Escobar Sandstone or Alhambra Formation, or from the Neogene units in Block IV. Recent geologic maps of the area include the Alhambra Formation within the Escobar Sandstone (Graymer and others, 1994a, 2002a). The San Ramon Sandstone unconformably overlies the Alhambra Formation and is interpreted as Oligocene based on the molluscan fauna (Schmidt, 1958). The Sobrante Sandstone disconformably overlies the San Ramon Sandstone in the Pacheco syncline and was interpreted as Relizian or lower Luisian Stage based on scarce molluscan assemblages and lithology (Schmidt, 1958). The overlying Briones Formation in the Pacheco syncline is considered Mohnian in age based on two microfossil samples (Schmidt, 1958) that contain benthic foraminifers. 
Table 19. Benthic foraminifers from the Muir Sandstone in Block IV.

[Taxonomy of species is given in the section entitled "Taxonomic Notes." $\mathrm{X}$ indicates the species is present. Samples that are barren of microfossils or contain few to rare diagnostic species include: Mf7771, Mf7772, and Mf7774]

\begin{tabular}{|c|c|}
\hline $\begin{array}{l}\text { Benthic foraminifers from the Muir } \\
\text { Sandstone in Block IV }\end{array}$ & $\frac{\stackrel{N}{N}}{N^{N}}$ \\
\hline Amphimorphina californica & $\mathrm{X}$ \\
\hline Amphimorphina ignota & $\mathrm{x}$ \\
\hline Bathysiphon eocenicus & $\mathrm{X}$ \\
\hline Bathysiphon santecruis & $\mathrm{x}$ \\
\hline Bulimina alazaensis & $x$ \\
\hline Bulimina macilenta & $x$ \\
\hline Bulimina trinitatensis & $x$ \\
\hline Cibicidoides eocaenus & $x$ \\
\hline Cibicidoides subspiratus & $x$ \\
\hline Cyclammina spp. & $\mathrm{x}$ \\
\hline Dentalina colei & $x$ \\
\hline Dentalina communis & $x$ \\
\hline Dentalina globulicauda & $x$ \\
\hline Eggerella elongata & $x$ \\
\hline Ellipsoglandulina multicostata & $\mathrm{x}$ \\
\hline Eponides lodoensis & $x$ \\
\hline Fursenkoina spp. & $\mathrm{x}$ \\
\hline Globobulimina pacifica & $\mathrm{X}$ \\
\hline Globocassidulina globosa & $x$ \\
\hline Gyroidina orbicularis & $\mathrm{X}$ \\
\hline Haplophragmoides obliquicameratus & $x$ \\
\hline Karreriella elongata & $\mathrm{X}$ \\
\hline Lenticulina inornata & $x$ \\
\hline Lenticulina spp. & $\mathrm{x}$ \\
\hline Lenticulina ulatisensis & $\mathrm{x}$ \\
\hline Marginulina subbullata & $\mathrm{x}$ \\
\hline Nodosaria deliciae & $\mathrm{x}$ \\
\hline Nodosaria longiscata & $\mathrm{x}$ \\
\hline Nodosaria spp. & $\mathrm{x}$ \\
\hline Nodosarella advena & $x$ \\
\hline Nuttaloides truempyi & $x$ \\
\hline Oridorsalis umbonatus & $x$ \\
\hline Osangularia mexicana & $x$ \\
\hline Planulina truncana & $\mathrm{x}$ \\
\hline Pullenia eocenica & $x$ \\
\hline Pullenia quinqueloba & $x$ \\
\hline Silicosigmoilina californica & $\mathrm{X}$ \\
\hline Stilostomella advena & $x$ \\
\hline Textularia adalta & $\mathrm{X}$ \\
\hline Trochammina globigeriniformis & $\mathrm{X}$ \\
\hline Vulvulina curta & $\mathrm{X}$ \\
\hline
\end{tabular}




\section{Block V}

Block V is bounded by the Calaveras Fault to the north and west, and by the Concord and Diablo Faults to the east. The southern portion of this block extends into Santa Clara and San Joaquin Counties where it is identified as the Mount Hamilton block of Wentworth (1993). This block also corresponds to a portion of the Livermore block of Buising and Walker (1995) and most of the Diablo block of Graham and others (1984). The Eocene Domengine Formation rests unconformably on the Cretaceous Great Valley sequence. Unconformably overlying the Paleogene is the Neogene San Pablo Group (Cierbo Sandstone and Neroly Formation), the Green Valley and Tassajara Formations of Conduit (1938), and the Livermore Gravel (fig. 13).

A spot sample (Mf8522) from rocks tentatively identified as the Cretaceous unit Kss by Graymer and others (1994a, 1996) contains a meager arenaceous benthic foraminiferal assemblage (table 20). This assemblage, which includes Cribrostomoides trinitatensis, Glomospira charoides, Hormosina spp., Karreriella spp., Silicosigmoilina californica, and Trochamminoides contortus, is most similar to Eocene assemblages near the Paleocene/Eocene boundary. This assemblage has almost no species in common with the Domengine Formation, but has numerous species in common with the lower Eocene unit Tes (Block I) of Graymer and others (1994a, 1996) and the Meganos Formation (Block VI). An explanation of this occurrence is not clear as the sample was not taken near a fault.

Fourteen microfossil samples were examined from the olive gray mudstone member of the Domengine Formation (table 20). Nine samples contained foraminiferal assemblages dominated by Eocene species that first appear in the early Eocene, including Bulimina alazaensis, B. macilenta, Cibicidoides coalingensis, C. eocaenus, Eponides mexicanus, and Karreriella chapapotensis. The early middle Eocene age is, however, based on the last appearance of Anomalina regina, Hanzawaia mauriciensis, Vaginulinopsis nudicostata, V. vacavillensis, and V. verruculosa, and the first or restricted appearances of Bulimina microcostata, Cibicidoides subspiratus, Eggerella elongata, and Lenticulina ulatisensis. These assemblages are assigned to the Ulatisian Stage and are considered coeval with planktic foraminiferal zones P10 through P14. Deposition occurred at lower middle bathyal depths (1,500-2,000 m) based on the presence of Cibicidoides coalingensis, Karreriella elongata, and various species of Stilostomella and Uvigerina hispida. Middle bathyal depths are also supported by the common occurrences of Anomalina regina, Bulimina macilenta, B. microcostata, K. chapapotensis, and Osangularia. Considerable material was transported from the shelf.

A single microfossil sample, Mf8835, was examined from the Neogene strata of Block V (table 21). This sample was taken from strata identified as the Cierbo Sandstone (Graymer and others, 1994a). The foraminiferal assemblage is long-ranging and suggests an Oligocene to Miocene age. Deposition occurred at middle bathyal depths (500-2,000 m) based on the upper depth limits of Pullenia miocenica, although neritic species dominate the assemblage.

Vertebrate remains and K/Ar dates from the Green Valley and Tassajara Formations of Conduit (1938) and the Livermore Gravel indicate these units are late Miocene to early Pliocene and Pliocene to Pleistocene in age (Richley, 1948; Pelletier, 1951; Savage, 1951, 1955; Sarna-Wojcicki, 1976; Wagner, 1978; Graymer and others, 1994b, 1996). 


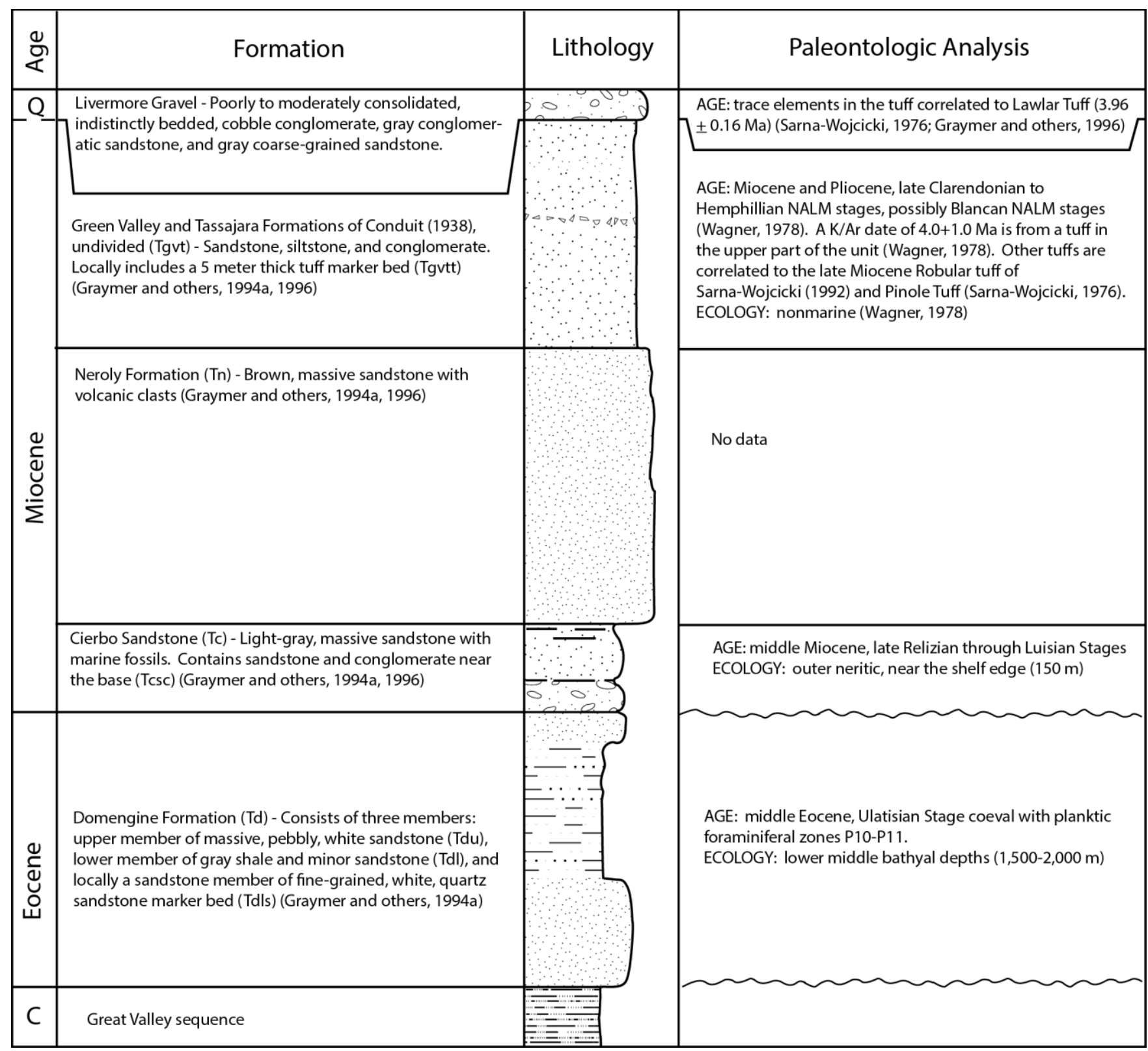

Figure 13. Composite columnar section for Block $V$. Age and environmental interpretations are summarized from the text. Q, Quaternary; C, Cretaceous. 
Table 20. Benthic foraminifers from the Domengine Formation and unit Kss of Graymer and others (1994a, 1996) in Block V.

[Taxonomy of species is given in the section entitled "Taxonomic Notes." X indicates the species is present. Samples that are barren of microfossils or contain few to rare diagnostic species include: Mf7830, Mf8838, Mf8840, Mf8841, Mf8842, Mf8996, and Mf9077. Sample Mf5007 from the Domengine Formation is upper Maastrichtian]

\begin{tabular}{|c|c|c|c|c|c|c|c|c|c|c|}
\hline \multirow{2}{*}{$\begin{array}{l}\text { Benthic foraminifers from the } \\
\text { Domengine Formation and } \\
\text { unit Kss of Graymer and } \\
\text { others (1994a, 1996) in Block } \\
\qquad\end{array}$} & $\stackrel{\mathscr{W}}{\mathscr{x}}$ & \multicolumn{9}{|c|}{ Domengine Formation } \\
\hline & $\begin{array}{l}\text { NN } \\
\text { N్} \\
\infty \\
\sum^{\infty}\end{array}$ & 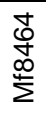 & $\begin{array}{l}10 \\
\text { N } \\
\infty \\
1 \\
\Sigma\end{array}$ & 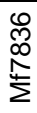 & $\begin{array}{l}\hat{N} \\
0 \\
\stackrel{1}{2} \\
\sum\end{array}$ & 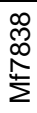 & 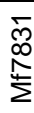 & $\begin{array}{l}\text { N } \\
\text { N } \\
\infty \\
N \\
\stackrel{\Sigma}{\Sigma}\end{array}$ & 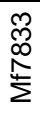 & 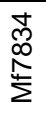 \\
\hline Allomorphina macrostoma & - & - & - & - & - & - & $\mathrm{x}$ & - & - & - \\
\hline Ammobacculites spp. & - & - & - & - & - & - & - & $X$ & $\mathrm{x}$ & - \\
\hline Ammodiscus incertus & - & - & - & - & $X$ & - & - & $X$ & $\mathrm{x}$ & $\mathrm{X}$ \\
\hline Anomalina keenae & - & - & $\mathrm{X}$ & $\mathrm{x}$ & $x$ & $\mathrm{x}$ & $\mathrm{x}$ & $x$ & $\mathrm{x}$ & $\mathrm{X}$ \\
\hline Anomalina regina & - & - & - & $\mathrm{X}$ & - & - & $\mathrm{X}$ & $X$ & - & - \\
\hline Anomalinoides spp. & $X$ & - & - & - & - & - & - & - & - & - \\
\hline Bathysiphon eocenicus & - & - & $\mathrm{X}$ & - & $X$ & - & $\mathrm{x}$ & $X$ & $\mathrm{X}$ & $\mathrm{X}$ \\
\hline Bathysiphon santecruis & $X$ & - & - & - & - & - & $\mathrm{X}$ & $X$ & $\mathrm{X}$ & - \\
\hline Bathysiphon spp. & $X$ & $X$ & $\mathrm{X}$ & $\mathrm{X}$ & $X$ & - & $\mathrm{X}$ & $X$ & $\mathrm{X}$ & $\mathrm{X}$ \\
\hline Bolivina jacksonensis & - & - & - & - & - & - & - & - & $\mathrm{X}$ & - \\
\hline Bulimina alazaensis & - & - & - & - & $X$ & - & - & $X$ & - & - \\
\hline Bulimina macilenta & - & - & - & - & $X$ & - & - & $X$ & $\mathrm{x}$ & - \\
\hline Bulimina microcostata & - & - & - & $x$ & $x$ & - & $x$ & $x$ & $x$ & - \\
\hline Chilostomella oolina & - & - & - & $\mathrm{X}$ & - & - & $\mathrm{X}$ & $X$ & $\mathrm{X}$ & - \\
\hline Chrysalongonium elongatum & - & - & - & $\mathrm{X}$ & - & - & - & - & $\mathrm{X}$ & - \\
\hline Chrysalongonium laeve & - & - & - & - & - & - & $\mathrm{X}$ & - & $\mathrm{X}$ & - \\
\hline Cibicides felix & - & - & $\mathrm{X}$ & - & - & - & - & - & - & - \\
\hline Cibicidoides coalingensis & - & - & - & $\mathrm{x}$ & $\mathrm{X}$ & $\mathrm{X}$ & $x$ & $x$ & - & - \\
\hline Cibicidoides eocaenus & - & - & $\mathrm{x}$ & - & - & - & - & - & $\mathrm{X}$ & $\mathrm{X}$ \\
\hline Cibicidoides subspiratus & - & - & $\mathrm{x}$ & $\mathrm{X}$ & $\mathrm{X}$ & $\mathrm{X}$ & $\mathrm{x}$ & $\mathrm{X}$ & $\mathrm{X}$ & $\mathrm{X}$ \\
\hline Cibicidoides spp. & - & - & $\mathrm{X}$ & $\mathrm{X}$ & - & - & - & - & $\mathrm{x}$ & - \\
\hline Cibicidoides venzuelanus & - & - & $\mathrm{X}$ & - & $\mathrm{X}$ & - & - & - & $\mathrm{x}$ & $\mathrm{X}$ \\
\hline Clavulina anglica & - & - & - & - & - & - & - & - & - & $X$ \\
\hline Cribrostomoides trintatensis & $\mathrm{X}$ & - & - & - & - & - & - & - & - & - \\
\hline Cyclammina pacifica & - & - & $\mathrm{X}$ & $\mathrm{x}$ & $X$ & $\mathrm{X}$ & $\mathrm{X}$ & $X$ & - & - \\
\hline Cyclammina samanica & $\mathrm{X}$ & - & - & - & - & - & - & - & - & - \\
\hline Cyclammina simiensis & - & - & $\mathrm{X}$ & - & - & - & - & - & $\mathrm{X}$ & $\mathrm{X}$ \\
\hline Cyclammina spp. & - & - & - & - & $x$ & $\mathrm{x}$ & - & - & - & - \\
\hline Dentalina basiplanata & - & - & - & - & - & - & - & - & $\mathrm{X}$ & - \\
\hline Dentalina colei & - & - & $\mathrm{X}$ & $\mathrm{X}$ & - & $\mathrm{X}$ & $\mathrm{x}$ & $\mathrm{X}$ & $\mathrm{X}$ & $\mathrm{X}$ \\
\hline Dentalina communis & - & - & - & - & $X$ & - & - & - & - & - \\
\hline Dentalina consobrina & - & - & - & $\mathrm{X}$ & - & - & - & - & - & - \\
\hline Dentalina delicatula & - & - & $x$ & - & - & - & - & - & $x$ & $x$ \\
\hline Dentalina jacksonensis & - & - & - & - & $X$ & $\mathrm{X}$ & $\mathrm{X}$ & - & - & - \\
\hline Eggerella elongata & - & - & - & - & $x$ & - & $\mathrm{x}$ & $\mathrm{X}$ & $\mathrm{x}$ & - \\
\hline Eggerella cf. E. elongata & - & - & - & - & - & - & - & - & - & - \\
\hline Eggerella subconica & - & - & $\mathrm{X}$ & - & $X$ & - & $\mathrm{X}$ & $X$ & $\mathrm{X}$ & $X$ \\
\hline Elphidium californicum & - & - & - & $\mathrm{x}$ & - & - & - & - & - & - \\
\hline Eponides lodoensis & - & - & $\mathrm{x}$ & $\mathrm{x}$ & $X$ & - & - & $X$ & $\mathrm{x}$ & - \\
\hline Eponides mexicanus & - & - & - & $\mathrm{X}$ & - & $\mathrm{x}$ & $\mathrm{x}$ & $\mathrm{X}$ & - & - \\
\hline Gaudryina coalingensis & - & - & - & $\mathrm{X}$ & $X$ & $\mathrm{X}$ & $\mathrm{X}$ & $X$ & $x$ & - \\
\hline
\end{tabular}




\begin{tabular}{|c|c|c|c|c|c|c|c|c|c|c|}
\hline \multirow{2}{*}{$\begin{array}{l}\text { Benthic foraminifers from the } \\
\text { Domengine Formation and } \\
\text { unit Kss of Graymer and } \\
\text { others (1994a, 1996) in Block } \\
\text { V }\end{array}$} & \multirow{2}{*}{$\begin{array}{l}\stackrel{\mathscr{W}}{\not} \\
\stackrel{N}{N} \\
\stackrel{\infty}{\infty} \\
\sum_{\Sigma}^{\infty}\end{array}$} & \multicolumn{9}{|c|}{ Domengine Formation } \\
\hline & & 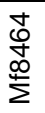 & 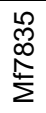 & $\begin{array}{l}0 \\
\mathbb{N} \\
\infty \\
\stackrel{\infty}{\Sigma} \\
\sum\end{array}$ & $\begin{array}{l}\hat{N} \\
\infty \\
\infty \\
\stackrel{N}{\Sigma}\end{array}$ & 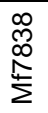 & $\begin{array}{l}\bar{m} \\
\infty \\
\stackrel{\infty}{\Sigma} \\
\sum\end{array}$ & 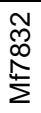 & 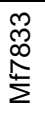 & \begin{tabular}{l} 
\} $\\
{\text { D }} \\
{\stackrel{1}{1}} \\
{\sum}$ \\
\hline Gaudryina laevigata & - & - & - & - & - & $x$ & - & $\mathrm{X}$ & - & - \\
\hline Gaudryina spp. & - & - & - & - & - & - & - & $x$ & - & - \\
\hline Glomospira charoides & $x$ & - & - & - & - & - & - & - & - & - \\
\hline Guttulina spp. & - & - & - & - & - & - & - & $x$ & - & - \\
\hline Gyroidina octocameratus & - & - & - & - & $x$ & - & - & - & $x$ & - \\
\hline Gyroidina planulata & - & - & $x$ & $x$ & - & $x$ & - & $x$ & $x$ & $x$ \\
\hline Gyroidina soldanii & - & - & - & - & - & - & $x$ & - & - & - \\
\hline Hanzawaia mauricensis & - & - & - & - & - & - & - & - & - & $x$ \\
\hline Haplophragmoides eggeri & - & - & - & - & - & - & $x$ & - & - & - \\
\hline Haplophragmoides spp. & - & $x$ & $x$ & $x$ & - & - & $x$ & $x$ & $x$ & $x$ \\
\hline Hormosina spp. & $x$ & - & - & - & - & - & - & - & - & - \\
\hline Hyperammina elongata & - & - & - & - & - & - & - & - & $x$ & - \\
\hline Karreriella chapapotensis & - & - & - & - & - & - & - & - & $x$ & - \\
\hline Karreriella elongata & - & - & - & - & - & - & $x$ & - & $x$ & $x$ \\
\hline Karreriella spp. & $x$ & - & - & - & - & - & - & - & - & - \\
\hline Lenticulina altolimbatus & - & - & $x$ & - & $x$ & $x$ & $x$ & $x$ & $x$ & $x$ \\
\hline Lenticulina caritae & - & - & $x$ & $x$ & $x$ & $x$ & - & $x$ & $x$ & $x$ \\
\hline Lenticulina convergens & - & - & - & - & - & - & $x$ & $x$ & - & - \\
\hline Lenticulina gyroscalpum & - & - & - & - & - & - & - & - & - & $x$ \\
\hline Lenticulina cf. L. gyroscalpum & - & - & - & - & - & $x$ & - & - & - & - \\
\hline Lenticulina limbosa hockleyensis & - & - & - & - & - & $x$ & - & - & $x$ & $x$ \\
\hline Lenticulina pseudocultratus & - & - & $x$ & - & $x$ & $x$ & $x$ & $x$ & - & $x$ \\
\hline Lenticulina pseudovortex & - & - & $x$ & $x$ & $x$ & $x$ & - & $x$ & $x$ & $x$ \\
\hline Lenticulina spp. & - & - & $x$ & $x$ & $x$ & $x$ & $x$ & $x$ & $x$ & $x$ \\
\hline Lenticulina terryi & - & - & - & - & - & - & - & - & - & $x$ \\
\hline Lenticulina ulatisensis & - & - & - & - & - & - & - & - & - & $x$ \\
\hline Lenticulina vortex & - & - & $x$ & - & - & - & $x$ & $x$ & - & - \\
\hline Marginulina subbullata & - & - & - & $x$ & - & $x$ & - & $x$ & $x$ & $x$ \\
\hline Nodosaria deliciae & - & - & - & - & $x$ & - & - & $x$ & $x$ & - \\
\hline Nodosaria latejugata & - & - & $x$ & $x$ & - & $x$ & $x$ & $x$ & $x$ & $x$ \\
\hline Nodosaria longiscata & - & - & - & $x$ & $x$ & $x$ & - & - & $x$ & $x$ \\
\hline Osangularia mexicana & - & - & - & $x$ & - & $x$ & $x$ & $x$ & $x$ & $x$ \\
\hline Pseudonodosaria conica & - & - & $x$ & - & - & $x$ & $x$ & - & $x$ & $x$ \\
\hline Praeglobobulimina pyrula & - & - & - & - & - & - & $x$ & - & - & - \\
\hline Pyrulina cylindroides & - & - & - & - & - & - & - & $x$ & - & - \\
\hline Reophax pilulifera & - & - & - & - & - & - & $x$ & - & - & - \\
\hline Silicosigmoilina californica & $\mathrm{x}$ & - & - & - & - & - & - & - & - & - \\
\hline Siphonia jacksonensis & - & - & - & $\mathrm{X}$ & - & $x$ & - & - & - & - \\
\hline Spiroplectammina richardi & $x$ & - & $x$ & - & $x$ & $x$ & $x$ & $x$ & $x$ & $x$ \\
\hline Stilostomella adolphina & - & - & - & - & - & - & - & $x$ & - & - \\
\hline Stilostomella gracilis & - & - & - & - & $x$ & $x$ & - & - & - & - \\
\hline Stilostomella lepidula & - & - & - & - & $x$ & $x$ & $x$ & - & $x$ & - \\
\hline Textularia adalta & - & - & - & - & $x$ & - & - & - & - & - \\
\hline Trifarina advena californica & - & - & - & - & - & - & - & - & $x$ & - \\
\hline Tritaxilina cole $i$ & - & - & - & - & - & - & - & - & $\mathrm{X}$ & - \\
\hline Trochammina globigeriniformis & - & - & $x$ & $x$ & $\mathrm{X}$ & - & $x$ & $\mathrm{X}$ & $x$ & $x$ \\
\hline
\end{tabular}
\end{tabular}




\begin{tabular}{|c|c|c|c|c|c|c|c|c|c|c|}
\hline \multirow{2}{*}{$\begin{array}{l}\text { Benthic foraminifers from the } \\
\text { Domengine Formation and } \\
\text { unit Kss of Graymer and } \\
\text { others (1994a, 1996) in Block } \\
\text { V }\end{array}$} & ڤ్ & \multicolumn{9}{|c|}{ Domengine Formation } \\
\hline & 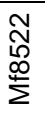 & $\begin{array}{l}\text { J } \\
\text { \$े } \\
\sum^{\circ}\end{array}$ & 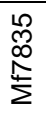 & 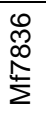 & $\begin{array}{l}\hat{N} \\
\infty \\
\stackrel{\infty}{2} \\
\mathbb{L}^{\prime}\end{array}$ & 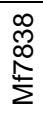 & $\begin{array}{l}\bar{m} \\
\infty \\
\stackrel{\infty}{p} \\
\sum\end{array}$ & 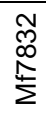 & 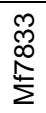 & 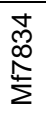 \\
\hline Trochamminoides contortus & $x$ & - & - & - & - & - & - & - & - & - \\
\hline Uvigerina hispida & - & - & - & - & - & - & - & - & $x$ & $x$ \\
\hline Vaginulinopsis asperuliformis & - & - & - & - & - & $x$ & - & - & $x$ & $x$ \\
\hline Vaginulinopsis nudicostata & - & - & $x$ & $x$ & $x$ & $x$ & $x$ & $x$ & $x$ & - \\
\hline Vaginulinopsis spp. & - & - & - & - & $x$ & - & - & - & - & - \\
\hline Vaginulinopsis vacavillensis & - & - & - & $x$ & - & $x$ & $x$ & $x$ & - & - \\
\hline Vaginulinopsis verruculosa & - & - & - & - & - & $\mathrm{x}$ & - & - & - & $\mathrm{x}$ \\
\hline
\end{tabular}

Table 21. Benthic foraminifers from the Cierbo Sandstone in Block V.

[Taxonomy of species is given in the section entitled "Taxonomic Notes." $\mathrm{X}$ indicates the species is present. Samples that are barren of microfossils or contain few to rare diagnostic species include: Mf5010, Mf5011, Mf5012, Mf5013, Mf8836, Mf8837, and Mf8839.]

\begin{tabular}{|l|c|}
\hline Benthic foraminifers from & \llcorner \\
the Cierbo Sandstone of & $\infty$ \\
Block V & $\stackrel{\infty}{\Sigma}$ \\
\hline Epistominella subperuviana & $\mathrm{X}$ \\
Fursenkoina californiensis & $\mathrm{X}$ \\
Nonionella costifera & $\mathrm{X}$ \\
Nonionella miocenica & $\mathrm{X}$ \\
Pullenia miocenica & $\mathrm{X}$ \\
\hline
\end{tabular}

\section{Block VI}

Block VI lies east of the Concord, Diablo, Greenville, and Carnegie Faults and extends beyond the boundaries of Contra Costa and Alameda Counties (fig. 1). This block corresponds to a portion of the East Coast Ranges block of Buising and Walker (1995) and part of the Diablo block of Graham and others (1984). The Tertiary section rests unconformably on the Cretaceous Great Valley sequence (fig. 14). The Paleogene section includes unit Tmz of Graymer and others (1994a), the Meganos Formation, Domengine Formation, Kreyenhagen Formation (Nortonville Shale, lower Markley Sandstone, Sidney Flat Shale, and upper Markley Sandstone Members), and Kirker Tuff. The Neogene section that unconformably overlies the Paleogene includes the San Pablo Group (Cierbo and Neroly sandstones), Oro Loma Formation, Lawlor Tuff, and Tulare Formation, and ranges from assumed Miocene to Pleistocene in age (fig. 14). Only one sample (Mf8523) was examined from the Neogene units in Block VI and it did not contain foraminifers.

Unit Tmz , the oldest Paleogene unit, is locally divided into an upper siltstone and shale member and a lower glauconitic sandstone member. Three fossiliferous samples (Mf7983, Mf7984, and Mf7985) from this unit contain a benthic foraminiferal assemblage (table 22) that is late Paleocene in age and assigned to the Ynezian Stage, coeval with planktic foraminiferal zone P4. The late Paleocene age is based on Anomalinoides rubiginosus, Cibicidoides dayi, and Uvigerina laimingi, as well as U.S. West Coast and Gulf Coast species such as Ammobacculites paleocenica, Anomalinoides welleri, Cibicidoides eponidiformis, Citharina plummerae, Lenticulina midwayensis, Nodosaria macneili, Pseudoglandulina manifesta, and V. tuberculata (table 22). Calcareous nannofossils from sample Mf7984 suggest a middle Paleocene age, and are questionably assigned to zone CP4 (Bukry and others, 
1998), which is coeval with planktic foraminiferal zone P4 and the Ynezian Stage. The benthic foraminiferal species present are common to slope and abyssal plain environments (Berggren and Aubert, 1976; van Morkhoven and others, 1986) but are not restricted to specific depths. Deposition is therefore believed to have occurred at bathyal depths with additional material transported from the shelf. Sample Mf7986, also from unit Tmz, contains arenaceous foraminifers that suggest and early Eocene or older age. Silicosigmoilina californica, Spiroplectammina directa, and Tritaxilina colei first appear in the Late Cretaceous or Paleocene and last appear in the early Eocene. These species are most common around the Paleocene/Eocene boundary in California.

The Meganos Formation is locally divided into four members, oldest to youngest: A, C, D, and E (Clark, 1921). Seven samples were examined from this formation (table 22): two from Tmc (Mf7981 and Mf7982), division C of Clark (1921); and four from Tme (Mf7974, Mf7975, Mf7976, and Mf7987), division E of Clark (1921). The lower (Tma) and third (Tmd) members of the Meganos Formation are sandstones and conglomerates (Graymer and others, 1994a, b) and were not sampled for microfossils.

Samples from member $\mathrm{C}$ of the Meganos Formation are early Eocene in age and assigned to the Penutian Stage. Most species in these assemblages are long-ranging forms that first appear in the Eocene such as Anomalinoides capitatus, Bulimina alazaensis, and Cibicidoides venezuelanus.

However, the presence of Clavulina anglica and Eponides lodoensis restrict the age of this assemblage to the early Eocene, Penutian Stage. Calcareous nannofossils are early Eocene in age, and assigned to zone CP9b (Bukry and others, 1998), which is coeval with the early Penutian Stage and planktic foraminiferal zone P6b. Deposition occurred at lower bathyal to abyssal depths ( $\geq 2,000 \mathrm{~m})$.

Samples from the member $\mathrm{E}$ of the Meganos Formation are late early Eocene in age and are assigned to the late Penutian Stage, coeval with planktic foraminiferal zone P9. The early Eocene age of these assemblages is based on a poorly developed form of Cibicidoides $\mathrm{cf}$. C. laurisae (typical C. laurisae first appear in P10), Cibicidoides subspiratus, Clavulina anglica, Eponides lodoensis, and Vaginulinopsis nudicostata. The presence of Siphonia wilcoxensis supports the late early Eocene age, and calcareous nannofossils also suggest an early Eocene age and are assigned to zone CP11 (Bukry and others, 1998). Deposition occurred at upper bathyal depths (150-500 m).

Two fossiliferous samples from the lower Domengine Formation contain Karreriella conversa and Silicosigmoilina californica, which indicate an age range of Cretaceous through early Eocene, and questionably to middle Eocene (table 22). Other species present are not diagnostic of age. Deposition occurred at bathyal to abyssal depths ( $\geq 150 \mathrm{~m})$. 


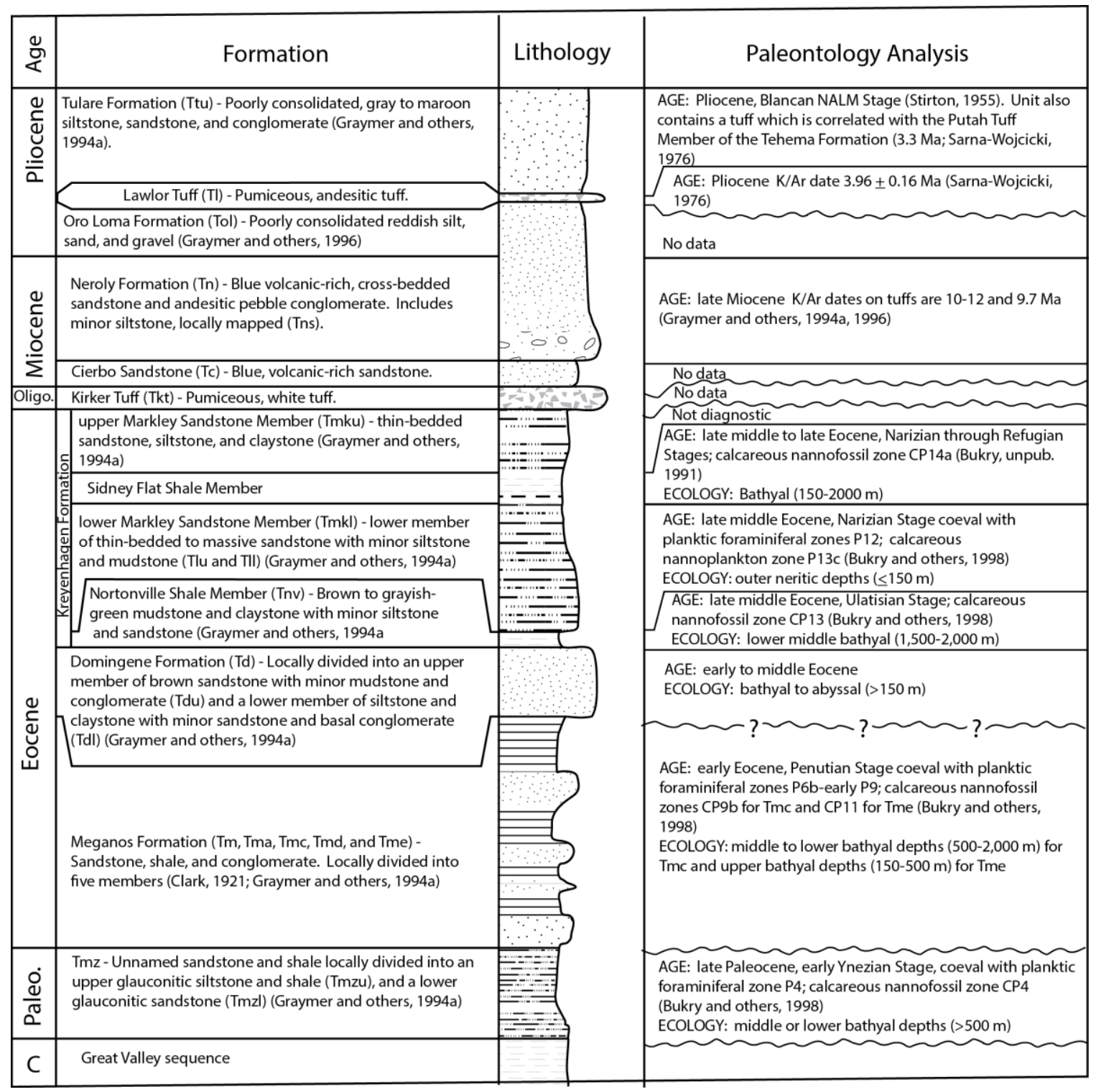

Figure 14. Composite columnar section for Block VI. Age and environmental interpretations are summarized from the text. Oligo., Oligocene; Paleo., Paleocene; C, Cretaceous. 
Table 22. Benthic foraminifers from Paleogene units of Block VI.

[Taxonomy of species is given in the section entitled "Taxonomic Notes." $\mathrm{X}$ indicates the species is present. Samples that are barren of microfossils or contain few to rare diagnostic species include: Mf7973, Mf7989, Mf8070, Mf8071, and Mf8454, all from Nortonville Shale; Mf7977, Mf7978, and Mf7980, Sidney Flat Shale; Mf7972, Mf7990, Mf8054, Mf8055, Mf8056, Mf8057, Mf8061, Mf8062, Mf8064, Mf8065, Mf8138, Mf8330, Mf8331, Mf8332, Mf8333, Mf8334, Mf8335, Mf8336, Mf8337, Mf8338, and Mf8414, Markley Sandstone. No faunal data available for samples Mf2044, Mf2045, and Mf2046, Markley Formation. Tmz, unnamed sandstone and shale (Graymer and others, 1994a); Tdl, lower Domengine Formation; Tnv, Nortonville Shale Member of the Kreyenhagen Formation; Tmk, Markley Sandstone Member of the Kreyenhagen Formation ; Tmkl, lower Markely Sandstone Member of the Kreyenhagen Foramtion; Tmku, upper Markley Sandstone Member of the Kreyenhagen Formation]

\begin{tabular}{|c|c|c|c|c|c|c|c|c|c|c|c|c|c|c|c|c|c|c|c|c|}
\hline \multirow{3}{*}{$\begin{array}{l}\text { Benthic foraminifers from } \\
\text { Paleogene units of Block VI }\end{array}$} & \multirow{2}{*}{\multicolumn{4}{|c|}{$\operatorname{Tmz}$}} & \multicolumn{6}{|c|}{ Meganos Formation } & & & \multicolumn{8}{|c|}{ Kreyenhagen Formation } \\
\hline & & & & & \multicolumn{2}{|c|}{ Tmc } & \multicolumn{4}{|c|}{ Tme } & \multicolumn{2}{|c|}{ Tdl } & $\vec{E}$ & \multicolumn{2}{|c|}{ Tmkl } & \multicolumn{4}{|c|}{ Sidney Flat Shale } & \multirow{2}{*}{ 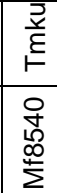 } \\
\hline & $\begin{array}{l}\mathscr{O} \\
\stackrel{o}{2} \\
\Sigma \\
\Sigma\end{array}$ & 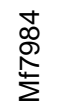 & $\begin{array}{l}\mathscr{L} \\
\infty \\
o \\
\mathbb{P} \\
\Sigma\end{array}$ & $\begin{array}{l}0 \\
\infty \\
o \\
\mathbb{P} \\
\Sigma\end{array}$ & $\begin{array}{l}\bar{\infty} \\
o \\
\stackrel{N}{\Sigma} \\
\end{array}$ & $\begin{array}{l}\text { o } \\
o \\
\stackrel{P}{\Sigma} \\
\sum\end{array}$ & $\begin{array}{l}\text { 索 } \\
\text { S } \\
\text { 足 }\end{array}$ & 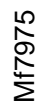 & 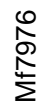 & $\begin{array}{l}\hat{D} \\
o \\
\mathbb{N} \\
\dot{L}\end{array}$ & $\begin{array}{l}\stackrel{8}{0} \\
\$ \\
\infty \\
\Sigma\end{array}$ & $\begin{array}{l}\overline{6} \\
\infty \\
\infty \\
\Sigma\end{array}$ & $\begin{array}{l}\tilde{N} \\
\stackrel{N}{\infty} \\
\stackrel{\infty}{\Sigma}\end{array}$ & $\begin{array}{l}\infty \\
\infty \\
o \\
N \\
\sum\end{array}$ & $\begin{array}{l}\mathbb{8} \\
0 \\
\infty \\
\sum\end{array}$ & $\begin{array}{l}\text { R } \\
\text { o } \\
\text { P } \\
\text { L }\end{array}$ & $\begin{array}{l}\text { o } \\
0 \\
\infty \\
\infty^{\infty}\end{array}$ & 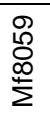 & $\begin{array}{l}8 \\
\& \\
\$ 0 \\
\sum\end{array}$ & \\
\hline Alabamina wilcoxensis & - & - & - & - & - & - & $x$ & - & - & - & - & - & - & - & - & - & - & - & - & - \\
\hline Ammobacculites paleocenica & - & $x$ & $\mathrm{x}$ & - & - & - & - & - & - & - & - & - & - & - & - & - & - & - & - & - \\
\hline Ammodiscus incertus & - & - & - & - & - & - & - & - & - & - & - & $\mathrm{x}$ & - & - & - & - & - & - & - & - \\
\hline Amphimorphina becki & - & - & - & - & - & - & - & - & - & - & - & - & $x$ & - & - & - & - & - & - & - \\
\hline Amphimorphina ignota & - & - & - & - & - & - & - & - & - & - & - & - & $\mathrm{x}$ & - & - & - & $\mathrm{x}$ & - & - & - \\
\hline Anomalinoides capitatus & - & - & - & - & $\mathrm{x}$ & $\mathrm{x}$ & - & - & - & - & - & - & - & - & - & - & - & - & - & - \\
\hline Anomalinoides rubiginosus & - & $x$ & $\mathrm{x}$ & - & - & - & - & - & - & - & - & - & - & - & - & - & - & - & - & - \\
\hline Anomalinoides welleri & - & $x$ & - & - & - & - & - & - & - & - & - & - & - & - & - & - & - & - & - & - \\
\hline Anomalina garzaensis & - & - & - & - & - & - & - & $\mathrm{x}$ & - & - & - & - & - & - & - & - & - & - & - & $x$ \\
\hline Anomalina keenae & - & - & - & - & - & $\mathrm{x}$ & $\mathrm{x}$ & - & - & - & - & - & - & - & - & - & - & - & - & - \\
\hline Anomalina spp. & - & - & - & - & $\mathrm{x}$ & - & $\mathrm{x}$ & - & - & - & - & - & - & - & - & - & - & - & - & - \\
\hline Bathysiphon eоcenicus & $\mathrm{x}$ & $\mathrm{x}$ & - & $x$ & $x$ & - & $x$ & $x$ & - & $x$ & - & - & $\mathrm{x}$ & - & - & - & - & - & - & - \\
\hline Bathysiphon santecruis & - & - & - & $\mathrm{x}$ & - & - & - & - & - & - & $x$ & $\mathrm{x}$ & - & - & - & - & - & - & - & - \\
\hline Bathysiphon spp. & $\mathrm{x}$ & - & $x$ & $x$ & - & $x$ & - & - & - & - & $x$ & $x$ & - & - & - & - & - & - & - & - \\
\hline Bolivina kleinpelli & - & - & - & - & - & - & - & - & - & - & - & - & - & - & - & - & - & - & - & $x$ \\
\hline Bulimina alazaensis & - & - & - & - & $\mathrm{x}$ & - & - & - & - & - & - & - & $\mathrm{x}$ & - & - & - & - & - & - & - \\
\hline Bulimina sculptilis & - & - & - & - & - & - & - & - & - & - & - & - & $\mathrm{x}$ & $x$ & - & - & - & - & - & - \\
\hline Bulimina spp. & - & - & - & - & - & - & - & - & - & - & - & - & - & - & $\mathrm{x}$ & - & - & - & - & - \\
\hline Caucasina schencki & - & - & - & - & - & - & - & - & - & - & - & - & - & $x$ & - & $x$ & - & - & - & - \\
\hline Ceratobulimina spp. & - & - & - & - & - & - & - & - & - & - & - & - & - & - & - & - & $\mathrm{x}$ & - & - & - \\
\hline Chilostomella ovoidea & - & - & - & - & - & - & - & - & - & - & - & - & $\mathrm{x}$ & - & - & - & - & - & - & - \\
\hline Chrysalongonium elongatum & - & - & - & - & - & - & - & $\mathrm{x}$ & - & - & - & - & - & - & - & - & - & - & - & - \\
\hline Cibicides martinezensis & - & - & - & - & - & - & - & - & - & - & - & - & $x$ & - & - & - & - & - & - & - \\
\hline Cibicidoides dayi & - & $\mathrm{x}$ & - & - & - & - & - & - & - & - & - & - & - & - & - & - & - & - & - & - \\
\hline Cibicidoides eocaenus & - & - & - & - & - & - & - & $\mathrm{x}$ & - & - & - & - & - & - & - & - & - & - & - & - \\
\hline Cibicidoides eponidiformis & - & $x$ & $x$ & - & - & - & - & - & - & - & - & - & - & - & - & - & - & - & - & - \\
\hline Cibicidoides cf. C. laurisae & - & - & - & - & - & - & $x$ & $x$ & - & - & - & - & - & - & - & - & - & - & - & - \\
\hline Cibicidoides subspiratus & - & - & - & - & - & - & $\mathrm{x}$ & $x$ & - & - & - & - & $x$ & - & - & - & - & - & - & - \\
\hline Cibicidoides spp. & - & - & - & - & - & - & - & $x$ & - & - & - & - & - & - & - & - & - & - & - & - \\
\hline Cibicidoides venzuelanus & - & - & - & - & $\mathrm{x}$ & - & - & $x$ & $\mathrm{x}$ & - & - & - & - & - & - & - & - & - & - & - \\
\hline Citharina plummoides & - & $\mathrm{x}$ & - & - & - & - & - & - & - & - & - & - & - & - & - & - & - & - & - & - \\
\hline Clavulina anglica & - & - & - & - & $\mathrm{x}$ & - & $\mathrm{x}$ & - & - & - & - & 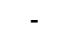 & - & - & - & - & - & - & - & - \\
\hline Cribrostomoides trintatensis & - & - & - & - & - & - & - & - & - & - & $\mathrm{x}$ & $x$ & - & - & - & - & - & - & - & - \\
\hline Cyclammina pacifica & - & - & - & - & - & - & - & - & - & - & - & - & $\mathrm{x}$ & - & - & - & - & - & - & - \\
\hline Cyclammina samanica & $\mathrm{x}$ & - & - & $\mathrm{x}$ & - & $\mathrm{x}$ & - & - & - & $\mathrm{x}$ & $\mathrm{x}$ & $\mathrm{x}$ & - & - & - & - & - & - & - & - \\
\hline Dentalina colei & - & - & - & - & - & - & - & $x$ & - & - & - & - & - & - & - & - & - & - & - & - \\
\hline Dentalina comтunis & - & - & - & - & - & - & - & - & - & 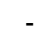 & - & - & $\mathrm{x}$ & - & - & - & - & - & - & - \\
\hline Dentalina pseudobliquestriata & - & $x$ & $\mathrm{x}$ & - & - & - & - & - & - & - & - & - & - & - & - & - & - & - & - & - \\
\hline Dentalina spp. & - & $x$ & $x$ & - & - & - & - & - & - & - & - & - & - & - & - & - & - & - & - & - \\
\hline Dorothia bulletta & $x$ & - & - & - & - & - & - & - & - & $x$ & - & - & - & - & - & - & - & - & & 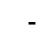 \\
\hline
\end{tabular}




\begin{tabular}{|c|c|c|c|c|c|c|c|c|c|c|c|c|c|c|c|c|c|c|c|c|}
\hline \multirow{3}{*}{$\begin{array}{l}\text { Benthic foraminifers from } \\
\text { Paleogene units of Block } \mathrm{VI}\end{array}$} & \multirow{2}{*}{\multicolumn{4}{|c|}{$\operatorname{Tmz}$}} & \multicolumn{6}{|c|}{ Meganos Formation } & & & \multicolumn{8}{|c|}{ Kreyenhagen Formation } \\
\hline & & & & & \multicolumn{2}{|c|}{ Tmc } & \multicolumn{4}{|c|}{ Tme } & \multicolumn{2}{|c|}{ Tdl } & $\vec{E}$ & \multicolumn{2}{|c|}{ Tmkl } & \multicolumn{4}{|c|}{ Sidney Flat Shale } & \multirow{2}{*}{ 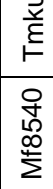 } \\
\hline & $\begin{array}{l}\text { m } \\
\infty \\
o \\
\mathbb{N} \\
\Sigma\end{array}$ & 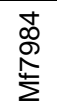 & 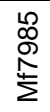 & 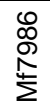 & $\begin{array}{l}\bar{D} \\
o \\
\stackrel{N}{\Sigma} \\
\Sigma\end{array}$ & $\begin{array}{l}\widetilde{N} \\
\stackrel{\infty}{P} \\
\stackrel{P}{\Sigma}\end{array}$ & 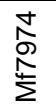 & $\begin{array}{l}\text { م) } \\
\text { S } \\
\stackrel{1}{\Sigma}\end{array}$ & $\begin{array}{l}0 \\
\stackrel{2}{\alpha} \\
\mathbb{R} \\
\dot{\Sigma}\end{array}$ & $\begin{array}{l}\hat{N} \\
\text { o } \\
\stackrel{N}{\Sigma} \\
\end{array}$ & $\begin{array}{l}\stackrel{0}{0} \\
\vdots \\
\frac{1}{\Sigma}\end{array}$ & $\begin{array}{l}\bar{\varphi} \\
\bar{\infty} \\
\Sigma \\
\dot{\infty}\end{array}$ & $\begin{array}{l}\hat{N} \\
\stackrel{N}{\infty} \\
\stackrel{\infty}{\Sigma}\end{array}$ & $\begin{array}{l}\infty \\
\infty \\
o \\
\stackrel{N}{N} \\
\sum\end{array}$ & $\begin{array}{l}\mathbb{0} \\
\varnothing \\
\infty \\
\sum^{\infty}\end{array}$ & $\begin{array}{l}\text { D } \\
\text { o } \\
\stackrel{1}{\Sigma}\end{array}$ & 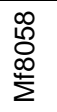 & $\begin{array}{l}\text { D } \\
\stackrel{0}{0} \\
\dot{\infty} \\
\dot{\Sigma}\end{array}$ & 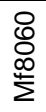 & \\
\hline Eggerella elongata & - & - & - & - & - & - & - & - & - & - & - & - & - & - & - & - & - & $\mathrm{x}$ & - & - \\
\hline Elphidium spp. & - & - & - & - & - & - & - & $x$ & - & - & - & - & - & - & & - & - & - & - & - \\
\hline Eponides lodoensis & - & - & - & - & $\mathrm{x}$ & - & - & $x$ & - & - & - & - & - & - & & - & - & - & - & - \\
\hline Eponides mexicanus & - & - & - & - & - & - & - & $x$ & - & - & - & - & - & - & - & - & - & - & - & - \\
\hline Fursenkoina bramletti & - & - & - & - & - & - & $\mathrm{x}$ & - & - & - & - & - & $x$ & $\mathrm{x}$ & - & - & - & - & $x$ & - \\
\hline Gaudryina coalingensis & - & $x$ & - & - & - & - & - & - & - & - & - & - & - & - & - & - & - & - & - & - \\
\hline Gaudryina laevigata & - & $\mathrm{x}$ & - & - & - & - & - & - & - & - & - & - & - & - & - & - & - & - & - & - \\
\hline Gaudryina pachecoensis & - & $\mathrm{x}$ & $\mathrm{X}$ & - & - & - & - & - & - & - & - & - & - & - & - & - & - & - & - & - \\
\hline Gaudryina spp. & - & - & $\mathrm{x}$ & - & - & - & - & - & - & - & - & - & - & - & - & - & - & - & - & - \\
\hline Gyroidina planulata & - & - & - & - & - & - & - & $x$ & - & - & - & - & - & - & - & - & - & - & - & - \\
\hline Gyroidina soldanii & - & - & - & - & - & - & - & - & - & - & - & - & $\mathrm{x}$ & - & - & - & - & - & - & - \\
\hline Hanzawaia blanpiedi & & $x$ & - & . & - & - & - & - & - & - & - & - & - & - & - & - & - & - & - & - \\
\hline Haplophragmoides eggeri & $\mathrm{x}$ & - & $x$ & $\mathrm{x}$ & $\mathrm{x}$ & - & - & - & - & $\mathrm{x}$ & $x$ & $\mathrm{x}$ & - & - & - & - & - & - & - & - \\
\hline Haplophragmoides spp. & - & - & - & $\mathrm{x}$ & - & $\mathrm{x}$ & - & - & - & - & $\mathrm{x}$ & - & $\mathrm{x}$ & - & - & - & - & $\mathrm{x}$ & - & - \\
\hline Hoeglundina eocenica & - & - & - & - & $\mathrm{x}$ & - & - & $\mathrm{x}$ & - & $\mathrm{x}$ & - & - & - & - & - & - & - & - & - & - \\
\hline Hormosina ovulum & - & - & - & - & - & - & - & - & - & - & - & $x$ & - & - & - & - & - & - & - & - \\
\hline Hyperammina elongata & . & - & - & - & - & - & - & - & - & - & $\mathrm{x}$ & $\mathrm{x}$ & - & - & - & - & - & - & - & - \\
\hline Karreriella conversa & - & - & - & - & - & - & - & - & - & - & - & $\mathrm{X}$ & - & - & - & - & - & - & - & - \\
\hline Karreriella elongata & - & - & - & - & - & - & - & - & - & - & - & - & $\mathrm{x}$ & - & - & - & - & - & - & - \\
\hline Karreriella spp. & - & - & - & - & - & - & - & - & - & - & $\mathrm{x}$ & - & - & - & - & - & - & - & - & - \\
\hline Lenticulina convergens & - & - & - & - & - & - & - & - & - & - & - & - & - & - & - & $\mathrm{x}$ & - & - & - & - \\
\hline Lenticulina midwayensis & - & $x$ & - & - & - & - & - & - & - & - & - & - & - & - & - & - & - & - & - & - \\
\hline Lenticulina pseudocultratus & - & - & - & - & - & - & - & - & - & - & - & - & $x$ & - & - & - & - & - & - & - \\
\hline Lenticulina pseudovortex & - & $\mathrm{x}$ & - & - & - & - & $\mathrm{x}$ & - & - & - & - & - & $\mathrm{x}$ & - & - & - & - & - & - & - \\
\hline Lenticulina spp. & - & $x$ & $x$ & - & $x$ & $x$ & $x$ & $x$ & - & $x$ & - & - & $x$ & - & - & - & - & - & - & - \\
\hline Lenticulina theta & - & - & - & - & - & - & - & - & - & - & - & - & - & - & - & $\mathrm{x}$ & - & - & - & - \\
\hline Lenticulina vortex & - & $\mathrm{x}$ & - & - & - & - & - & - & - & - & - & - & $\mathrm{x}$ & - & - & - & - & - & - & - \\
\hline Lenticulina welchi & - & - & - & - & - & - & - & - & - & - & - & - & $\mathrm{x}$ & - & - & - & - & - & - & - \\
\hline Marginulina exima & - & $\mathrm{x}$ & - & - & - & - & - & - & - & - & - & - & - & - & - & - & - & - & - & - \\
\hline Marginulina munda & - & - & - & - & - & - & - & - & - & - & - & - & - & - & - & $\mathrm{x}$ & - & - & - & - \\
\hline Marginulina subbullata & - & - & - & - & - & - & - & - & - & - & - & - & $x$ & - & - & - & - & - & - & - \\
\hline Marginulina spp. & - & - & $\mathrm{x}$ & - & - & - & - & - & - & - & - & - & - & - & - & - & - & $\mathrm{x}$ & - & $\mathrm{x}$ \\
\hline Nonionella spp. & - & - & - & - & - & - & $\mathrm{x}$ & - & - & - & - & - & - & - & - & - & - & - & - & $\mathrm{x}$ \\
\hline Nodosaria affinis & - & $\mathrm{x}$ & - & - & - & - & - & - & - & - & - & - & - & - & - & - & - & - & - & - \\
\hline Nodosaria deliciae & - & - & - & - & - & - & - & - & - & - & - & - & $\mathrm{x}$ & - & - & - & - & - & - & - \\
\hline Nodosaria longiscata & - & $x$ & $x$ & - & - & - & - & - & - & $x$ & - & - & $x$ & - & - & - & - & - & - & - \\
\hline Nodosaria macneili & - & $\mathrm{x}$ & $\mathrm{x}$ & - & - & - & - & - & - & - & - & - & - & - & - & - & - & - & - & - \\
\hline Nodosaria spp. & - & - & $\mathrm{x}$ & - & $\mathrm{x}$ & $\mathrm{x}$ & - & - & - & $\mathrm{x}$ & - & - & $\mathrm{x}$ & - & $\mathrm{x}$ & - & $\mathrm{x}$ & - & - & - \\
\hline Nonion halkyardi & - & - & - & - & - & - & - & - & - & - & - & - & $x$ & - & - & - & - & - & - & - \\
\hline Oridorsalis umbonatus & - & - & - & - & $\mathrm{x}$ & - & - & - & - & - & - & - & $\mathrm{x}$ & - & - & - & - & - & - & - \\
\hline Osangularia mexicana & - & - & - & - & - & - & - & - & $\mathrm{x}$ & - & - & - & $\mathrm{x}$ & - & - & - & - & - & - & - \\
\hline Pseudoglandulina manifesta & - & $\mathrm{x}$ & - & - & - & - & - & - & - & - & - & - & - & - & - & - & - & - & - & - \\
\hline Pseudonodosaria conica & - & - & - & - & $\mathrm{x}$ & - & $\mathrm{x}$ & - & - & - & - & - & - & - & - & - & - & - & - & - \\
\hline Pseudonodosaria inflata & - & - & - & - & - & - & - & - & - & - & - & - & $x$ & - & - & - & - & - & - & - \\
\hline Praeglobobulimina ovata & - & - & - & - & - & - & - & - & - & - & - & - & - & - & - & - & - & $\mathrm{x}$ & - & - \\
\hline Praeglobobulimina pupoides & 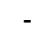 & - & - & - & $\mathrm{x}$ & - & - & - & 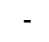 & . & - & - & $\mathrm{x}$ & - & - & - & $\mathrm{x}$ & - & - & - \\
\hline Praeglobobulimina pyrula & - & - & - & - & - & - & - & - & - & - & - & - & - & - & - & $\mathrm{x}$ & - & - & - & - \\
\hline Praeglobobulimina spp. & - & $\mathrm{x}$ & - & - & - & - & - & - & - & $\mathrm{x}$ & - & - & - & - & - & - & - & - & - & - \\
\hline Pullenia eocenica & - & - & - & - & - & - & - & - & - & - & - & - & $\mathrm{x}$ & - & - & - & - & - & - & - \\
\hline Quinqueloculina spp. & - & - & - & - & - & - & - & - & - & - & - & - & - & $\mathrm{x}$ & & - & - & - & - & - \\
\hline Quinqueloculina triangularis & & - & $\mathrm{X}$ & & - & & - & - & - & - & - & & 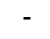 & - & & - & - & - & - & \\
\hline
\end{tabular}




\begin{tabular}{|c|c|c|c|c|c|c|c|c|c|c|c|c|c|c|c|c|c|c|c|c|}
\hline \multirow{3}{*}{$\begin{array}{l}\text { Benthic foraminifers from } \\
\text { Paleogene units of Block } \mathrm{VI}\end{array}$} & \multirow{2}{*}{\multicolumn{4}{|c|}{ Tmz }} & \multicolumn{6}{|c|}{ Meganos Formation } & & & \multicolumn{8}{|c|}{ Kreyenhagen Formation } \\
\hline & & & & & \multicolumn{2}{|c|}{ Tmc } & \multicolumn{4}{|c|}{ Tme } & \multicolumn{2}{|c|}{ Tdl } & $\geqq$ & \multicolumn{2}{|c|}{ Tmkl } & \multicolumn{4}{|c|}{ Sidney Flat Shale } & \multirow{2}{*}{ 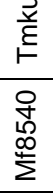 } \\
\hline & 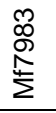 & 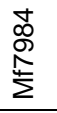 & 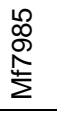 & $\begin{array}{l}\infty \\
\infty \\
⿱ 乛 \\
\stackrel{N}{\Sigma}\end{array}$ & 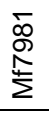 & 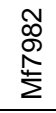 & $\begin{array}{l}\text { N } \\
\text { S } \\
\stackrel{N}{\Sigma}\end{array}$ & $\begin{array}{l}\stackrel{2}{\Lambda} \\
\stackrel{2}{R} \\
\sum\end{array}$ & $\begin{array}{l}0 \\
\hat{\Omega} \\
\stackrel{1}{\Sigma}\end{array}$ & $\begin{array}{l}\hat{\infty} \\
o \\
o \\
\sum \\
\sum\end{array}$ & 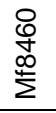 & $\begin{array}{l}\bar{o} \\
\Phi \\
\stackrel{\infty}{\Sigma}\end{array}$ & $\begin{array}{l}\mathbb{N} \\
\hat{o} \\
\sum^{\infty}\end{array}$ & 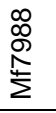 & 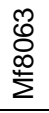 & 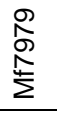 & $\begin{array}{l}\infty \\
\mathscr{l}^{\circ} \\
\infty^{\infty}\end{array}$ & $\begin{array}{l}\text { D } \\
\stackrel{0}{0} \\
\sum^{\infty}\end{array}$ & 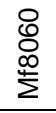 & \\
\hline Reophax spp. & - & - & $\mathrm{x}$ & - & - & - & - & - & - & - & - & - & - & - & - & - & - & - & - & - \\
\hline Saccammina complanata & - & - & - & - & - & - & - & - & - & - & - & $\mathrm{x}$ & - & - & - & - & - & - & - & - \\
\hline Saccammina spp. & - & - & - & - & - & - & - & - & - & - & $\mathrm{x}$ & - & - & - & - & - & - & - & - & - \\
\hline Saracenaria spp. & - & - & - & - & $\mathrm{x}$ & - & - & - & - & - & - & - & - & - & - & - & - & - & - & - \\
\hline Saracenaria triangularis & - & $\mathrm{x}$ & - & - & - & - & - & - & - & - & - & - & $\mathrm{x}$ & - & - & - & - & - & - & - \\
\hline Stichocassidulina spp. & - & - & - & - & - & - & - & - & - & - & - & - & - & - & - & - & $\mathrm{x}$ & - & - & - \\
\hline Silicosigmoilina californica & $\mathrm{x}$ & $\mathrm{x}$ & - & $\mathrm{x}$ & - & - & - & - & - & $\mathrm{x}$ & $\mathrm{x}$ & $\mathrm{x}$ & - & - & - & - & - & - & - & - \\
\hline Siphonia wilcoxensis & - & - & - & - & - & - & - & $\mathrm{x}$ & - & - & - & - & - & - & - & - & - & - & - & - \\
\hline Spiroplectammina directa & - & - & - & $\mathrm{x}$ & - & - & - & - & - & - & - & - & - & - & - & - & - & - & - & - \\
\hline Spiroplectammina richardi & - & $\mathrm{x}$ & $\mathrm{x}$ & - & - & - & - & $\mathrm{x}$ & - & $\mathrm{x}$ & - & - & - & - & - & - & - & - & - & - \\
\hline Stilostomella adolphina & - & - & - & - & - & - & - & - & - & - & - & - & - & $\mathrm{x}$ & - & - & - & - & - & $\mathrm{x}$ \\
\hline Stilostomella lepidula & - & - & - & - & $x$ & - & - & $\mathrm{x}$ & - & - & - & - & $\mathrm{x}$ & - & $\mathrm{x}$ & $\mathrm{x}$ & - & - & - & - \\
\hline Stilostomella spp. & - & - & - & - & - & - & $x$ & - & - & - & - & - & - & $\mathrm{x}$ & - & - & - & - & - & - \\
\hline Tritaxilina colei & - & - & - & $\mathrm{x}$ & $\mathrm{x}$ & - & - & - & - & - & - & - & - & - & - & - & - & - & - & - \\
\hline Tritaxia globulifera & - & $\mathrm{x}$ & $\mathrm{x}$ & - & - & - & - & - & - & - & - & - & - & - & - & - & - & - & - & - \\
\hline Trochammina globigeriniformis & - & - & - & - & $\mathrm{x}$ & - & - & - & - & - & - & - & - & - & - & - & - & - & - & - \\
\hline Uvigerina hispida & - & - & - & - & - & - & - & - & - & - & - & - & - & - & - & - & - & $\mathrm{x}$ & - & - \\
\hline Uvigerina laimingi & - & $\mathrm{x}$ & - & - & 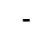 & - & - & - & - & - & - & - & - & - & - & - & - & - & - & - \\
\hline Uvigerina spp. & - & - & - & - & - & - & - & - & - & - & - & - & - & $\mathrm{x}$ & - & - & - & - & - & - \\
\hline Vaginulinopsis asperuliformis & - & - & - & - & - & - & - & - & - & $\mathrm{x}$ & - & - & - & - & - & - & - & - & - & - \\
\hline Vaginulinopsis nudicostata & - & - & - & - & - & - & - & $\mathrm{x}$ & - & - & - & - & - & - & - & - & - & - & - & - \\
\hline Vaginulinopsis spp. & - & - & - & - & - & - & $x$ & - & - & - & - & - & - & - & - & - & - & - & - & - \\
\hline Vaginulinopsis tuberculata & - & $\mathrm{x}$ & $\mathrm{x}$ & - & - & - & - & - & - & - & - & - & - & - & - & - & - & - & - & - \\
\hline Valvulineria chirana & - & - & - & - & - & - & - & - & - & - & - & - & - & - & - & - & - & - & - & $\mathrm{x}$ \\
\hline Valvulineria spp. & - & - & - & - & - & - & $x$ & - & - & - & - & - & - & - & - & - & - & - & - & - \\
\hline Valvulineria tumeyensis & - & - & - & - & - & - & - & - & - & - & - & - & - & $x$ & - & $\mathrm{x}$ & - & - & - & - \\
\hline Vulvulina curta & - & - & - & - & - & - & - & - & - & - & - & - & $\mathrm{x}$ & - & - & - & - & - & - & 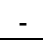 \\
\hline
\end{tabular}

A single fossiliferous sample, Mf8072, was from the Nortonville Shale Member of the Kreyenhagen Formation (table 22) and contains benthic foraminifers that suggest a late middle Eocene age, Ulatisian Stage. The late middle Eocene age is based on the overlapping ranges of Amphimorphina becki, Bulimina sculptilis, and Cibicidoides subspiratus. The presence of Lenticulina welchi suggests assignment to the late Ulatisian Stage, as does the abundance of Bulimina alazaensis. Calcareous nannoplankton suggest that this sample should be assigned to the early middle Eocene zone CP13 (Bukry and others, 1998), which is coeval with the late Ulatisian Stage. Sample Mf7989, also from the Nortonville Shale, contained abundant diatoms, radiolarians, and calcareous nannofossils, but only rare fragments of foraminifers. The calcareous nannofossils indicate a middle Eocene age and are assigned to zone CP13? (Bukry and others, 1998). Deposition occurred at lower middle bathyal depths (1,500-2,000 m) based on the presence of Bulimina alazaensis, Karreriella elongata, Stilostomella lepidula, and Vulvulina curta, which have upper depth limits in the lower middle bathyal biofacies.

Four samples (Mf7979, Mf8058, Mf8059, and Mf8060) from the Sidney Flat Shale Member of the Kreyenhagen Formation contain a sparse benthic foraminiferal assemblage that suggests a late middle to late Eocene age and is assigned to the Narizian through Refugian Stages, based on the occurrence of Caucasina schencki, Eggerella elongata, Stichocassidulina spp. (probably S. thalmani), and Valvulineria tumeyensis (table 22). Calcareous nannofossils from sample Mf7979 (Sidney Flat Shale Member) are late middle Eocene in age and assigned to zone CP14a (Bukry, 1991). The benthic 
foraminiferal assemblage suggests deposition occurred at bathyal depths. No age diagnostic species were identified in Mf7977, Mf7778, and Mf7980.

Samples from the Markley Sandstone contain some radiolarians and a few benthic foraminifers but are largely barren of microfossils. No age diagnostic assemblages were identified in 21 of the 24 samples. Sample Mf7988 from the lower part of the unit contains a sparse benthic foraminiferal assemblage that includes Bulimina sculptilis, Caucasina schencki, and Valvulineria tumeyensis. These species suggest the assemblage is late middle to late Eocene in age and the assemblage is thus assigned to the Narizian through Refugian Stages, coeval with planktic foraminiferal zones P12 through P17. Calcareous nannofossils from the same sample suggest an early middle Eocene age and are assigned to zone CP13c (Bukry and others, 1998), which is older than the age based on benthic foraminifers. Samples Mf8063 from the lower member and Mf 8540 from the upper member of the Markely Sandstone contained a few nondiagnostic foraminifers as well as abundant diatoms and radiolarians (table 22). Deposition occurred at outer neritic depths (<150 m).

Sample Mf8330 was taken at a depth of 33 feet in well P-11 (2,100 feet below the base of the tuffaceous sandstone of the Kirker Tuff, in the lower Markley Sandstone but well above the Nortonville Shale) and sample Mf8333 was taken at a depth of 113 to 121 feet in well MW-9 (estimated to be 1,000 feet stratigraphically above Mf8330). Both samples contain abundant fragments of a deep-water snail and a planktic foraminifer, Pseudohastigerina micra, which suggests a middle Eocene (P9) to early Oligocene (P18) age. Sample Mf8330 also contained several specimens of Bolivina rankini and Nonion multicamerata, which are indicative of the late Miocene Mohnian Stage and are believed to be downhole contamination. These species are probably from the overlying Neroly Formation that contains a tuff with K/Ar ages of about 10-12 and 9.7 Ma (A.M. Sarna-Wojcicki, cited in Graymer and others, 1994a, b, 1996).

Younger units are nonmarine and include the Oro Loma Formation, Lawlor Tuff and Tulare Formation. The Lawlor Tuff has a K/Ar age of 3.96 $\pm 0.16 \mathrm{Ma}$ (Sarna-Wojcicki, 1976; Graymer and others, 1994a); the Putah Tuff has a K/Ar age of $3.3 \pm 0.1 \mathrm{Ma}$, and the Tulare Formation contains a tuff that is correlated with the Putah Tuff (Graymer and others, 1994a; Sarna-Wojcicki, 1976).

\section{Block VII}

Block VII occurs in Alameda County between the Hayward Fault to the west and Miller Creek, Palomares, and Stonybrook Faults to the east (fig. 1). This block extends west into Santa Clara County where it has been identified as the Alum Rock block (Wentworth, 1993). The southeast boundary is the Calaveras Fault. Block VII corresponds to the southern portion of the East Bay Hills block of Buising and Walker (1995) and the southern part of the Caldecott block of Graham and others (1984). The Paleogene section of Block VII is composed of two formations: an unnamed unit (Tps) and the Tolman Formation (Tt). Both formations form small bodies that unconformably overlie the Upper Cretaceous part of the Great Valley sequence along the Chabot Fault (Graymer and others, 1996; R.W. Graymer, written commun., 2011). Unit Tps is late Paleocene in age. Two samples were examined from the Tolman Formation during this study; both are barren. The formation is considered Eocene in age based primarily on calcareous algae and is thought to represent nearshore deposition based on abundant barnacle remains (Hall, 1958). The Neogene section of Block VII is in fault contact with Paleogene strata. South of the study area in Santa Clara County, the Neogene strata unconformably overlie the Cretaceous Great Valley sequence (Wentworth and others, 1999). The Neogene section (fig. 15) includes, in ascending order, an unnamed shale unit (Tsh) of Graymer and others (1994a, 1996), Sobrante Sandstone, Claremont Shale, Oursan Sandstone, Tice Shale, Briones Sandstone, Orinda Formation, and an unnamed volcanic unit (Tv) of Graymer and others (1994a; Sarna-Wojcicki, 1976). 
Microfossil samples examined are predominantly from the Claremont Shale, Oursan Sandstone, and Tice Shale, but no samples were examined from the Sobrante Sandstone, only one sample was examined from the Briones Sandstone, and one from the unit Tsh of Graymer and others (1994a).

The unnamed Paleogene unit (Tps) is a dark gray, indistinctly to distinctly bedded siltstone, claystone, and shale, which grades downward into indistinctly bedded, dark brown to green, coarsegrained, glauconite-bearing lithic sandstone. Two samples (Mf7793 and Mf8194) from unit Tps contain a diverse benthic foraminiferal assemblage that is late Paleocene in age. Fourteen additional samples are barren or contain nondiagnostic arenaceous fauna (table 23). The late Paleocene age is based on numerous species that last appear near the Paleocene/Eocene boundary in planktic foraminiferal zones P5, P6a, or P6b, such as Anomalinoides rubiginosus, A. praeacutus, Bolivinoides delicatulus, Bulimina midwayensis, Cibicidoides alleni, $C$. hyphalus, C. praecursoria, $C$. velascoensis, Coryphostoma midwayensis, Gyroidinoides globosus, Neoeponides hillebrandti, Neoflabellina jarvisi, Osangularia velascoensis, Stensioina beccariiformis, and Tappanina selmensis. The presence of Alabamina wilcoxensis, Bulimina bradburyi, Eponides plummerae, Hanzawaia ammpiloba, Osangularia mexicana, and Trifarina advena californica, which first appear in the Paleocene near the boundary and range into the Eocene, limit the age of these assemblages to late Paleocene, probably coeval with planktic foraminiferal zones P5 or P6a. Calcareous nannoplankton in sample Mf7793 are assigned to the late Paleocene zone CP8 (Bukry and others, 1998) and thus support the foraminiferal age. Although this fauna is similar to middle bathyal assemblages, the presence of Gyroidinoides globosus, Silicosigmoilina californica, and various species of Pleurostomella suggest deposition occurred at lower bathyal depths $(1,500-2,000 \mathrm{~m})$. 


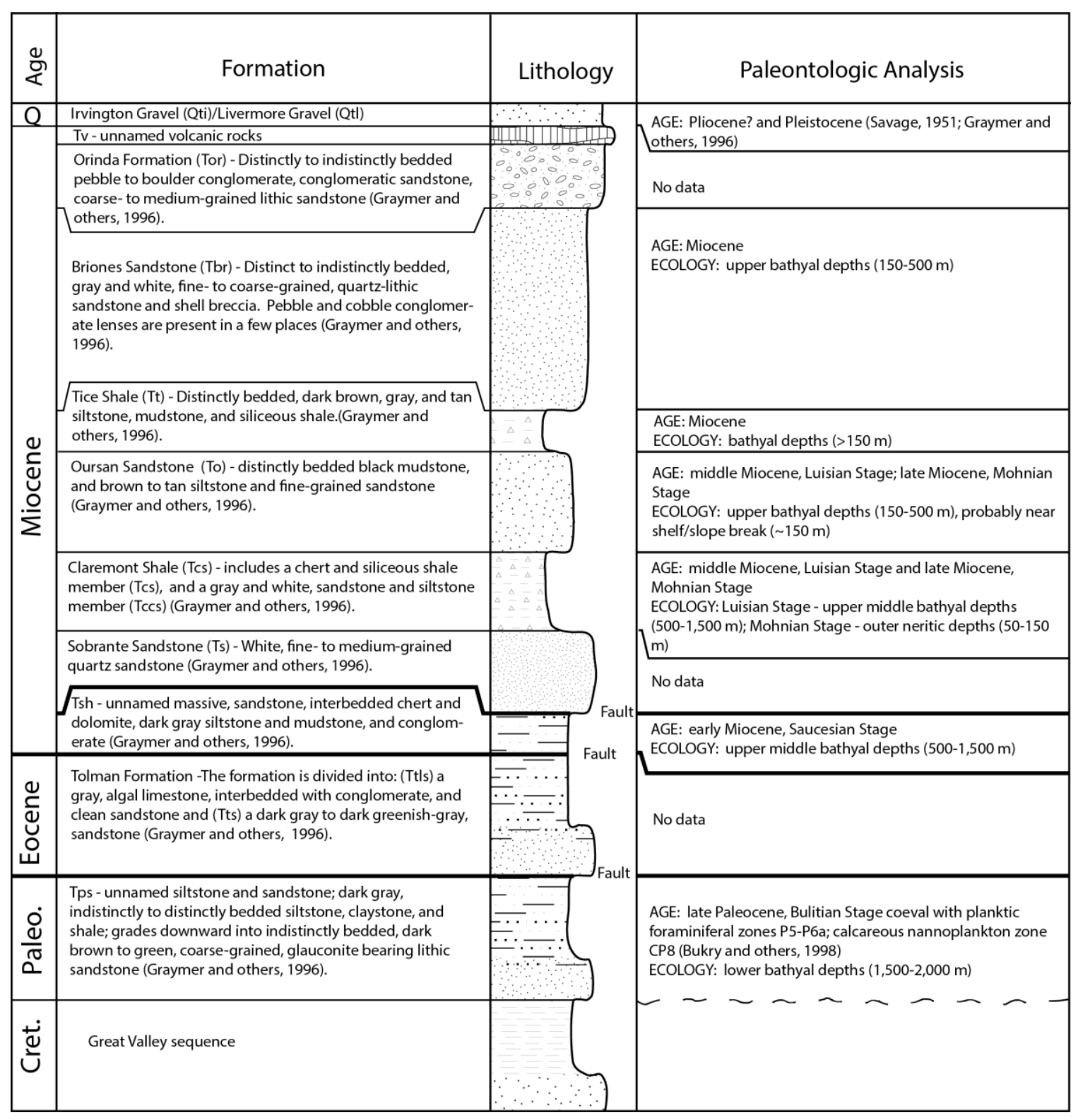

Figure 15. Composite columnar section for Block VII. Age and environmental interpretations are summarized from the text. Contact between the Cretaceous and the Tolman Formation is obscured (Graymer and others, 1996) but probably is unconformable (Graymer, written commun., 2011). The contacts between the Tolman Formation and the unnamed Tps unit and between the Tps unit and Cretaceous is not discussed in Graymer and others (1996). Q, Quaternary, Paleo., Paleocene, Cret., Cretaceous. 
Table 23. Benthic foraminifers from unit Tps of Graymer and others (1994a, 1996) in Block VII.

[Taxonomy of species is given in the section entitled "Taxonomic Notes." X indicates the species is present; cf. indicates that the specimens resemble or compare to the species. Samples that are barren of microfossils or contain few to rare diagnostic species include: Mf762, Mf765, Mf768, Mf770, Mf773, Mf774, Mf775, Mf791, Mf792 (A1), Mf792 (B1), Mf794, Mf7794, Mf7871, and Mf8122. Barren samples from the Tolman Formation Mf8040 and Mf8041]

\begin{tabular}{|c|c|c|}
\hline $\begin{array}{l}\text { Benthic foraminifers from unit } \\
\text { Tps of Graymer and others } \\
(1994 b, 1996) \text { in Block VII }\end{array}$ & 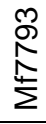 & 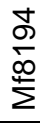 \\
\hline Alabamina wilcoxensis & $x$ & - \\
\hline Allomorphina conica & - & $x$ \\
\hline Ammobacculites expansus & - & $\mathrm{X}$ \\
\hline Ammobacculites paleocenica & - & $X$ \\
\hline Ammobacculites spp. & $x$ & - \\
\hline Ammodiscus pennyi & - & $X$ \\
\hline Anomalinoides praeacuta & $x$ & $\mathrm{X}$ \\
\hline Anomalinoides rubiginosus & $\mathrm{X}$ & - \\
\hline Anomalinoides welleri & $x$ & - \\
\hline Astacolus crepidulus & - & $\mathrm{X}$ \\
\hline Astacolus spp. & - & $\mathrm{X}$ \\
\hline Bathysiphon spp. & $x$ & $\mathrm{X}$ \\
\hline Bolivinoides delicatulus & $x$ & - \\
\hline Bolivina spp. & - & $\mathrm{X}$ \\
\hline Bulimina alazaensis & $x$ & - \\
\hline Bulimina bradburyi & - & $\mathrm{X}$ \\
\hline Bulimina midwayensis & $x$ & $\mathrm{X}$ \\
\hline Buliminella grata & $x$ & $x$ \\
\hline Chrysalongonium elongatum & $x$ & $X$ \\
\hline Chrysalongonium teniucostatum & - & $x$ \\
\hline Cibicidoides alleni & $x$ & - \\
\hline Cibicidoides hyphalus & $x$ & - \\
\hline Cibicidoides praecursorius & $x$ & - \\
\hline Cibicidoides praemundulus & - & $x$ \\
\hline Cibicidoides velascoensis & - & cf. \\
\hline Clavulina anglica & $x$ & - \\
\hline Coryphostoma midwayensis & - & $x$ \\
\hline Cribrostomoides trintatensis & - & $x$ \\
\hline Cyclammina samanica & - & $x$ \\
\hline Darbayella wilcoxensis & - & $x$ \\
\hline Dentalina communis & $x$ & - \\
\hline Dentalina spp. & $x$ & - \\
\hline Dorothia spp. & - & $x$ \\
\hline Eponides plummerae & $x$ & - \\
\hline Fissurina orbignyana & - & $\mathrm{X}$ \\
\hline Gaudryina laevigata & $x$ & - \\
\hline Gaudryina pyramidata & - & $x$ \\
\hline Globulina gibba & - & $\mathrm{X}$ \\
\hline Glomospira charoides & $x$ & - \\
\hline Guttulina problema & - & $\mathrm{X}$ \\
\hline Gyroidina globosus & - & $\mathrm{X}$ \\
\hline Gyroidina soldanii & $x$ & - \\
\hline Gyroidina spp. & - & $\mathrm{X}$ \\
\hline Hanzawaia ammophila & $x$ & - \\
\hline Hanzawaia blanpiedi & $x$ & - \\
\hline Haplophragmoides eggeri & $x$ & - \\
\hline Haplophragmoides glabra & - & $\mathrm{X}$ \\
\hline Haplophragmoides spp. & - & - \\
\hline
\end{tabular}

\begin{tabular}{|c|c|c|}
\hline $\begin{array}{l}\text { Benthic foraminifers from unit } \\
\text { Tps of Graymer and others } \\
\text { (1994b, 1996) in Block VII }\end{array}$ & $\begin{array}{l}\text { m } \\
\text { N } \\
\frac{N}{\Sigma}\end{array}$ & $\frac{\dot{\sigma}}{\frac{\sigma}{\infty}}$ \\
\hline Hoeglundina eocenica & $X$ & - \\
\hline Karreriella conversa & - & $\mathrm{X}$ \\
\hline Karreriella horrida & $x$ & - \\
\hline Lagena acuticosta & - & $x$ \\
\hline Lagena costata & $x$ & - \\
\hline Lagena laevis & $\mathrm{x}$ & - \\
\hline Lenticulina arcuatostriata & $x$ & - \\
\hline Lenticulina limbosa hockleyensis & $x$ & - \\
\hline Lenticulina pseudocultratus & $x$ & - \\
\hline Lenticulina pseudomammiligera & - & - \\
\hline Lenticulina pseudovortex & $x$ & $x$ \\
\hline Lenticulina spp. & $x$ & $\mathrm{X}$ \\
\hline Lenticulina vortex & $X$ & $X$ \\
\hline Lituotuba lituiformis & $x$ & $\mathrm{x}$ \\
\hline Loxostomoides applinae & $X$ & - \\
\hline Marginulina glabra & - & $x$ \\
\hline Marginulina munda & - & - \\
\hline Marginulina subbullata & - & $x$ \\
\hline Neoeponides hillebrandti & $X$ & - \\
\hline Neoflabellina jarvisi & - & $x$ \\
\hline Nonionella ovata & - & $\mathrm{X}$ \\
\hline Nonionella spp. & $\mathrm{X}$ & $\mathrm{X}$ \\
\hline Nodosaria limbata & - & $\mathrm{X}$ \\
\hline Nodosaria longiscata & $x$ & - \\
\hline Nodosaria macneili & $X$ & - \\
\hline Nodosaria velascoensis & - & $\mathrm{X}$ \\
\hline Nuttaloides truempyi & - & $x$ \\
\hline Oolina spp. & - & $\mathrm{X}$ \\
\hline Oridorsalis umbonatus & $x$ & $x$ \\
\hline Osangularia mexicana & $\mathrm{X}$ & - \\
\hline Osangularia velascoensis & - & $x$ \\
\hline Palmula primitiva & - & $\mathrm{X}$ \\
\hline Pseudoglandulina manifesta & $x$ & - \\
\hline Pleurostomella nuttalli & - & $X$ \\
\hline Pleurostomella spp. & $x$ & $\mathrm{X}$ \\
\hline Pullenia jarvis & $x$ & $\mathrm{X}$ \\
\hline Pyrulina cylindroides & $x$ & $\mathrm{X}$ \\
\hline Pyrulina spp. & - & $\mathrm{X}$ \\
\hline Quadrimorphina allomorphinoides & - & $\mathrm{X}$ \\
\hline Saccammina spp. & - & 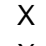 \\
\hline Saracenaria spp. & - & $\mathrm{X}$ \\
\hline Silicosigmoilina californica & $X$ & $\mathrm{X}$ \\
\hline Spiroplectammina directa & - & $\mathrm{X}$ \\
\hline Stensioina beccariformis & - & $\mathrm{X}$ \\
\hline Stilostomella spp. & - & $\mathrm{X}$ \\
\hline Tappanina selmensis & $\mathrm{X}$ & $\mathrm{X}$ \\
\hline Textularia conica & - & X \\
\hline Textularia plummerae & $x$ & $\mathrm{X}$ \\
\hline
\end{tabular}




\begin{tabular}{|c|c|c|}
\hline $\begin{array}{l}\text { Benthic foraminifers from unit } \\
\text { Tps of Graymer and others } \\
(1994 b, 1996) \text { in Block VII }\end{array}$ & $\stackrel{\substack{N \\
N}}{N}$ & $\begin{array}{l}\text { ठ } \\
\frac{\infty}{\Sigma} \\
\sum\end{array}$ \\
\hline Trifarina advena californica & $X$ & - \\
\hline Tritaxilina colei & - & $x$ \\
\hline Tritaxia globulifera & - & $x$ \\
\hline Tritaxia spp. & - & $x$ \\
\hline Trochammina globigeriniformis & - & cf. \\
\hline Trochammina spp. & $x$ & \\
\hline
\end{tabular}

\begin{tabular}{|c|c|c|}
\hline $\begin{array}{l}\text { Benthic foraminifers from unit } \\
\text { Tps of Graymer and others } \\
(1994 b, 1996) \text { in Block VII }\end{array}$ & $\begin{array}{l}\mathscr{M} \\
\stackrel{N}{N} \\
\stackrel{N}{\Sigma}\end{array}$ & \\
\hline Trochamminoides contortus & 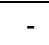 & \\
\hline Vaginulinopsis longiforma & - & \\
\hline Verneuilina triangulata & - & \\
\hline
\end{tabular}

Sample Mf7872 is from the oldest Neogene unit in Block VII, unit Tsh of Graymer and others (1994a, 1996), which crops out only as a small fault-bounded block near the mouth of Niles Canyon. Benthic foraminifers are moderately abundant in this sample and indicate an early Miocene age (table 24). The early Miocene age is constrained by the numerous species that last appear in the late Saucesian Stage (Bulimina inflata alligata, Hanzawaia crassiseptus, Siphogeneria transversa, and the uvigerinellids) and species that first appear in the late Saucesian Stage (Cassidulina panzana, Nonionella costifera, and N. miocenica). Except for a few shelf species (Nonionella costiferum and $N$. miocenica), the bulk of the benthic foraminifers have upper depth limits in the upper bathyal and upper middle bathyal biofacies, indicating that deposition occurred in the upper middle bathyal biofacies. Based on the common occurrence of Uvigerina rustica, water depths were probably closer to $1,500 \mathrm{~m}$ than $500 \mathrm{~m}$.

Microfossil samples from the overlying Claremont Shale include many barren or sparsely fossiliferous samples (table 24). The sparsely fossiliferous assemblages include long-ranging species, such as Fursenkoina californiensis and Nonionella costifera, which indicate a Miocene or younger age. Samples with age diagnostic assemblages suggest a middle Miocene age and are assigned to the Luisian Stage. This interpretation is based on the presence of Anomalina salinasensis, Bolivina advena striatella, Cassidulina panzana, C. pulchella, Globobulimina montereyana, G. pseudotorta, Plectofrondicularia advena, Pullenia miocenica, Uvigerina joaquensis, and Valvulineria californica. Deposition occurred at upper middle bathyal depths (1,500-2,000 m).

Two samples, Mf8125 and Mf8136, taken from rocks questionably assigned to the Claremont Shale, contain a sparse fauna that is indicative of the late middle Miocene Mohnian Stage (table 25). Age diagnostic species include Nonionella montereyana, which ranges from the Mohnian through Delmontian Stages. The fauna also suggests deposition occurred at outer neritic, possibly upper bathyal depths. This interpretation is not consistent with faunas from the Claremont Shale in this block, however, similar aged faunas were identified in the Claremont Shale samples in Block II near the Briones and San Pablo Reservoirs. 
Table 24. Benthic foraminifers from the Neogene units of Block VII.

[Taxonomy of species is given in the section entitled "Taxonomic Notes." $\mathrm{X}$ indicates the species is present. Samples that are barren of microfossils or contain few to rare nondiagnostic species include: Mf804, Mf1562A, Mf3268, Mf3357, Mf3358, Mf3359, Mf3360, Mf3361, Mf8034, Mf8035, Mf8039, Mf8043, Mf8132, Mf8133, Mf8134, Mf8135, Mf8187, Mf8188, Mf8191, Mf8200, Mf8201, Mf8320, Mf8422, Mf8423, Mf8424, Mf8425, and Mf8523. Samples with no report, slides or residue: Mf1657, Mf1658, and Mf1659]

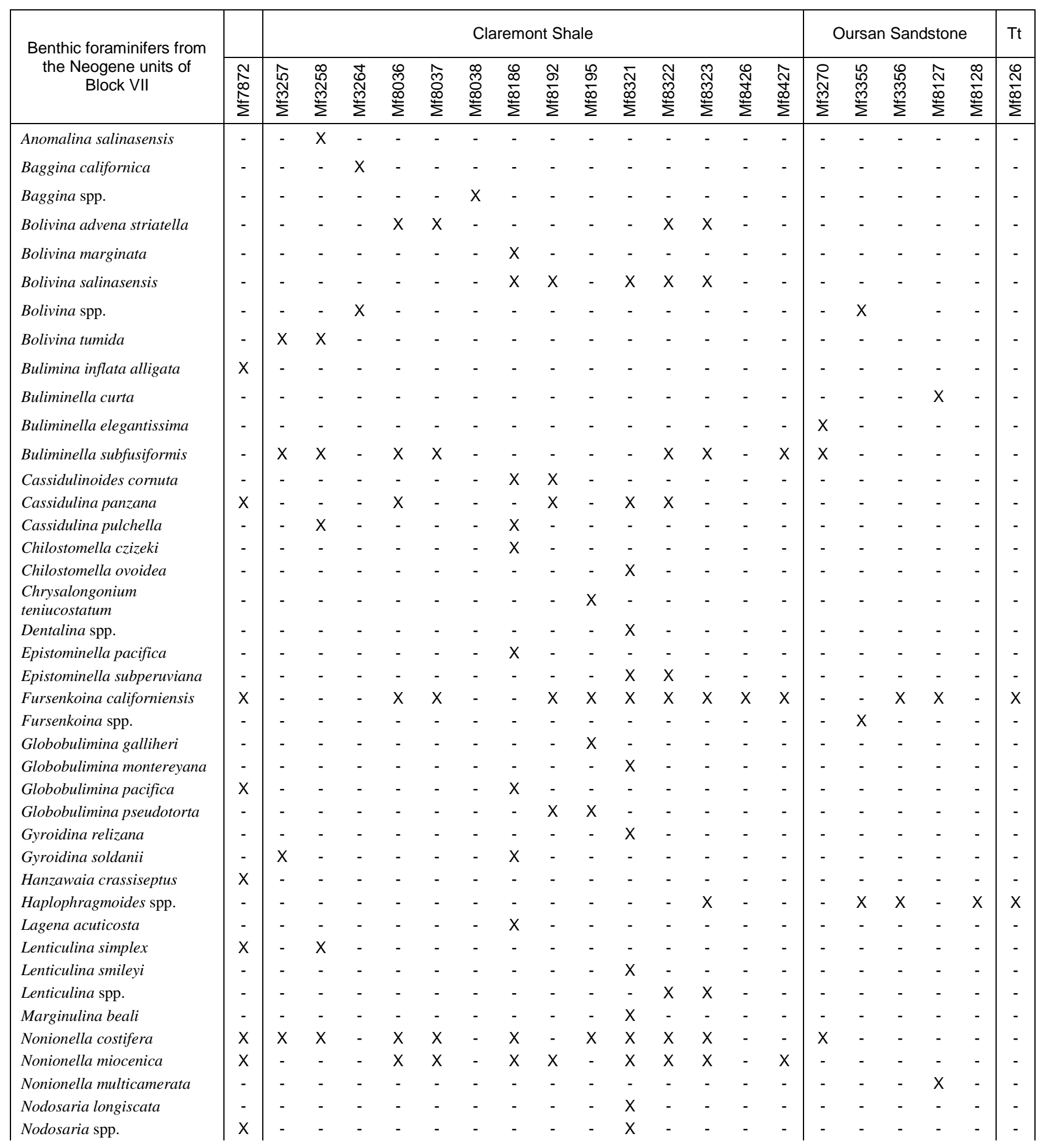




\begin{tabular}{|c|c|c|c|c|c|c|c|c|c|c|c|c|c|c|c|c|c|c|c|c|c|}
\hline \multirow{2}{*}{$\begin{array}{c}\text { Benthic foraminifers from } \\
\text { the Neogene units of } \\
\text { Block VII }\end{array}$} & & \multicolumn{14}{|c|}{ Claremont Shale } & \multicolumn{5}{|c|}{ Oursan Sandstone } & \multirow{2}{*}{ 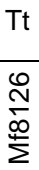 } \\
\hline & 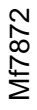 & $\stackrel{N}{\stackrel{N}{N}}$ & 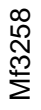 & 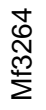 & 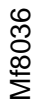 & $\begin{array}{l}\hat{n} \\
\stackrel{0}{\infty} \\
\stackrel{\Sigma}{\Sigma}\end{array}$ & 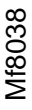 & $\begin{array}{l}\infty \\
\infty \\
\frac{\infty}{\infty} \\
\sum\end{array}$ & $\frac{\widetilde{\sigma}}{\frac{\infty}{\infty}}$ & $\begin{array}{l}\frac{1}{\infty} \\
\frac{\infty}{\Sigma}\end{array}$ & $\begin{array}{l}\bar{ָ} \\
\stackrel{\infty}{\infty} \\
\stackrel{\infty}{\Sigma}\end{array}$ & $\begin{array}{l}\text { N } \\
\text { } \\
\stackrel{\infty}{\Sigma}\end{array}$ & $\begin{array}{l}\text { ্ָ } \\
\stackrel{\infty}{\infty} \\
\stackrel{\infty}{\Sigma}\end{array}$ & 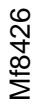 & 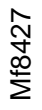 & 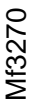 & $\begin{array}{l}\stackrel{1}{n} \\
\stackrel{m}{m} \\
\sum\end{array}$ & $\begin{array}{l}\mathscr{c} \\
\stackrel{1}{m} \\
\stackrel{M}{\Sigma}\end{array}$ & $\begin{array}{c}N \\
\stackrel{\infty}{\infty} \\
\sum\end{array}$ & $\begin{array}{l}\stackrel{\infty}{N} \\
\frac{\infty}{\infty} \\
\sum\end{array}$ & \\
\hline Oridorsalis umbonatus & $X$ & - & - & - & - & - & - & - & - & - & - & - & - & - & - & - & - & - & - & - & - \\
\hline Plectofrondicularia advena & - & - & - & - & - & - & - & - & - & - & - & - & $X$ & - & - & - & - & - & - & - & - \\
\hline Praeglobobulimina pupoides & $\mathrm{X}$ & - & - & - & - & - & - & - & - & - & - & - & - & - & - & - & - & - & - & - & - \\
\hline Pullenia miocenica & - & - & - & - & - & - & - & - & $X$ & - & - & - & - & - & - & - & - & - & - & - & - \\
\hline Siphogenerina spp. & - & - & - & - & - & - & - & - & $\mathrm{X}$ & - & - & - & - & - & - & - & - & - & - & - & - \\
\hline Siphogenerina transversa & $X$ & - & - & - & - & - & - & - & - & - & - & - & - & - & - & - & - & - & - & - & - \\
\hline Trifarina spp. & - & - & - & - & - & - & - & - & - & - & - & - & - & - & - & - & $X$ & - & - & - & - \\
\hline Uvigerinella californica & $\mathrm{X}$ & - & - & - & - & - & - & - & - & - & - & - & - & - & - & - & - & - & - & - & - \\
\hline Uvigerinella obesa impolita & $X$ & - & - & - & - & - & - & - & - & - & - & - & - & - & - & - & - & - & - & - & - \\
\hline Uvigerinella spp. & - & - & - & - & - & $X$ & - & - & - & - & - & - & - & - & - & - & $X$ & & - & - & - \\
\hline Uvigerina beccarii & $X$ & - & - & - & - & - & - & - & - & - & - & - & - & - & - & - & - & - & - & - & - \\
\hline Uvigerina gesteri & $X$ & - & - & - & - & - & - & - & - & - & - & - & - & - & - & - & - & - & - & - & - \\
\hline Uvigerina hispida & - & - & - & - & - & - & - & - & - & $X$ & - & - & - & - & - & - & - & - & - & - & - \\
\hline Uvigerina joaquinensis & - & - & - & - & - & - & - & $X$ & - & $X$ & - & - & $X$ & - & - & - & - & - & - & - & - \\
\hline Uvigerina rustica & $X$ & - & - & - & - & - & - & - & - & - & - & - & - & - & - & - & - & - & - & - & - \\
\hline Uvigerina spp. & - & - & - & - & - & - & - & - & - & - & - & $X$ & - & - & - & - & - & - & - & - & - \\
\hline Valvulineria araucana & $X$ & - & - & - & - & - & - & - & - & - & - & - & - & - & - & - & - & - & - & - & - \\
\hline Valvulineria californica s.s. & - & - & $X$ & - & - & - & - & - & - & - & $X$ & $X$ & - & - & - & - & - & - & - & - & - \\
\hline Valvulineria miocenica & - & $X$ & - & - & - & - & - & - & - & - & - & - & - & - & - & - & - & - & - & - & - \\
\hline Valvulineria spp. & - & - & - & - & - & - & - & - & - & - & - & - & - & - & - & $X$ & $X$ & - & - & - & - \\
\hline Valvulineria wilcoxensis & - & - & - & - & - & - & - & - & - & - & $X$ & - & - & - & - & - & - & - & - & - & - \\
\hline Virgulinella pertusa & - & - & - & - & - & - & - & - & - & - & $X$ & $X$ & - & - & - & - & - & - & - & - & - \\
\hline
\end{tabular}

Table 25. Benthic foraminifers from the Neogene Briones Sandstone and Claremont Shale(?) of Block VII.

[Taxonomy of species is given in the section entitled "Taxonomic Notes." $\mathrm{X}$ indicates the species is present. Samples that are barren of microfossils or contain few to rare nondiagnostic species include: Mf3271, Mf3358, Mf3362, Mf3363, and Mf8117. Samples with no report, slides or residue: Mf1660]

\begin{tabular}{|c|c|c|c|c|c|}
\hline \multirow{2}{*}{$\begin{array}{l}\text { Benthic foraminifers from } \\
\text { the Neogene Briones } \\
\text { Sandstone and Claremont } \\
\text { shale (?) of Block VII }\end{array}$} & \multicolumn{3}{|c|}{$\begin{array}{l}\text { Briones } \\
\text { Sandstone }\end{array}$} & \multicolumn{2}{|c|}{ Tcs? } \\
\hline & $\begin{array}{l}\text { o } \\
\text { N } \\
\Sigma\end{array}$ & $\begin{array}{l}\text { Y } \\
\text { D } \\
\Sigma\end{array}$ & 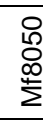 & 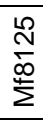 & $\begin{array}{l}\infty \\
\frac{m}{\infty} \\
\infty \\
\Sigma\end{array}$ \\
\hline Cibicides fletcheri & $\mathrm{x}$ & - & - & - & - \\
\hline Fursenkoina californiensis & - & $x$ & $\mathrm{x}$ & $\mathrm{x}$ & $\mathrm{x}$ \\
\hline Nonionella costifera & - & - & $\mathrm{x}$ & $x$ & - \\
\hline Nonionella miocenica & - & - & $\mathrm{x}$ & - & - \\
\hline Nonionella montereyana & - & - & - & $\mathrm{x}$ & $\mathrm{x}$ \\
\hline Tritaxilina colei & - & - & $\mathrm{x}$ & - & - \\
\hline
\end{tabular}


The Oursan Sandstone overlies the Claremont Shale in Block VII. Most microfossil samples from this unit contain a low diversity assemblage that indicates a Miocene age, including Buliminella curta, B. subfusiformis, Fursenkoina californiensis, and Nonionella costifera (table 24). One sample from the Oursan Sandstone, Mf8127, contains a single specimen of Nonionella multicamerata, which is restricted to the early Mohnian Stage and if correctly identified would indicate a late middle Miocene age. Although upper bathyal depths are suggested, deposition probably occurred near the shelf/slope break because many of the species present have upper depth limits that are transitional between the outer neritic and upper bathyal biofacies.

A single fossiliferous sample, Mf8126, from the Tice Shale contains only long-ranging Miocene species (table 24). The assemblage indicates deposition occurred at bathyal depths.

Although 10 samples were examined from the Briones Sandstone, only three contain microfossils (table 25). These assemblages are sparsely fossiliferous and contain only long-ranging species such as Fursenkoina californiensis, Nonionella costifera, and N. miocenica. Deposition occurred during the Miocene and at upper bathyal depths (150-500 m).

The younger formations in this block include the Orinda Formation, unnamed volcanic rocks of Graymer and others (1996), and the Irvington and Livermore gravels. The Irvington Gravel contains vertebrate fossils described by Savage (1951) as early Pleistocene (Graymer and others, 1996).

\section{Block IX}

Block IX is a small block in the southern part of Alameda County. It is bounded on the west by the Calaveras Fault and to the east and north by smaller faults (fig. 1). This block corresponds to a portion of the Livermore block of Buising and Walker (1995) and portions of the Diablo block of Graham and others (1984). In this block, Neogene strata unconformably overlie the Cretaceous Franciscan Complex mélange (Graymer and others 1994a, 1996). The Neogene units include the Temblor Formation, Claremont Shale, Oursan Sandstone, Tice Shale, and Briones Sandstone (fig. 16). No samples were examined from Temblor Formation in this block. This formation is considered to be Oligocene and Miocene at other locations in California (Hall, 1958; Addicott, 1973). Samples from the Tice Shale and Briones Sandstone overlying the Oursan Sandstone were barren of benthic foraminifers.

Five microfossil samples were examined from the Claremont Shale in Block IX: one was barren (Mf7863), and four (Mf7859, Mf7860, Mf7861, and Mf7862) contained species that indicate the middle Miocene Luisian Stage (table 26). Samples Mf7859 (base), Mf7860, and Mf7861 (top) are from a exposure of Claremont Shale on the southwest side of Calaveras Creek and sample Mf7862, although not in the same section, is interpreted to be stratigraphically below the other three samples (E.E. Brabb, written commun., 1990). In addition to long-ranging, nondiagnostic species, these samples include Valvulineria californica, Bolivina advena striatella, and Cassidulina panzana, which suggest the middle Miocene Luisian Stage. Deposition occurred at upper bathyal depths (150-500 m).

Three samples examined from the Oursan Sandstone (Mf1561A, Mf1560A, and Mf7866) contain Bolivina advena striatella, B. salinasensis, and Cassidulina panzana, which suggest a Miocene_-probably middle Miocene - age (table 26). Deposition occurred at upper bathyal depths near the shelf edge $(\sim 150 \mathrm{~m})$. 


\begin{tabular}{|c|c|c|c|}
\hline$\stackrel{0}{⿺ 辶}$ & Formation & Lithology & Paleontologic Analysis \\
\hline \multirow{5}{*}{ 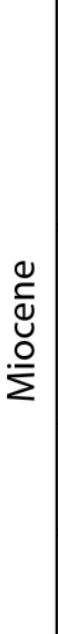 } & $\begin{array}{l}\text { Briones Sandstone (Tbr) - Distinct to indistinctly bedded, } \\
\text { gray and white, fine- to coarse-grained, quartz-lithic } \\
\text { sandstone and shell breccia. Pebble and cobble conglomer- } \\
\text { ate lenses are present in a few places (Graymer and others, } \\
\text { 1996). }\end{array}$ & & No data \\
\hline & $\begin{array}{l}\text { Tice Shale (Tt) - Thin, distinct beds of dark brown shale and } \\
\text { claystone (Graymer and others, 1996). }\end{array}$ & & No data \\
\hline & $\begin{array}{l}\text { Oursan Sandstone (To) - Indistinctly bedded, olive } \\
\text { sandstone, siltstone and claystone. Pebble and shell } \\
\text { fragment conglomerate near base (Graymer and others, } \\
\text { 1996). }\end{array}$ & & $\begin{array}{l}\text { AGE: middle Miocene } \\
\text { ECOLOGY: upper bathyal depths, near the shelf edge }(\sim 150 \\
\text { m) }\end{array}$ \\
\hline & $\begin{array}{l}\text { Claremont Shale (Tcc) - Distinctly bedded, massive, gray and } \\
\text { black, laminated siliceous shale. Locally interbedded, } \\
\text { calcareous sandstone and limestone (Graymer and others, } \\
\text { 1996). }\end{array}$ & & $\begin{array}{l}\text { AGE: middle Miocene, Luisian Stage } \\
\text { ECOLOGY: upper bathyal depths }(150-500 \mathrm{~m})\end{array}$ \\
\hline & $\begin{array}{l}\text { Temblor Formation (Ttem) - Thickly bedded and indistinctly } \\
\text { bedded sandstone and pebble conglomerate. }\end{array}$ & & No data \\
\hline 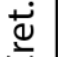 & Franciscan Complex Melange & & \\
\hline
\end{tabular}

Figure 16. Composite columnar section for Block IX. Age and environmental interpretations are summarized from the text. 
Table 26. Benthic foraminifers from the Claremont Shale and Oursan Sandstone of Block IX.

[Taxonomy of species is given in the section entitled "Taxonomic Notes." X indicates the species is present. Samples that are barren of microfossils or contain few to rare nondiagnostic species include: Mf7863, Mf7864, Mf7865, and Mf7867.

Samples with no report, slides or residue: Mf1653, Mf1654, Mf1655, Mf1661, Mf1663, Mf1664, and Mf1665]

\begin{tabular}{|c|c|c|c|c|c|c|c|}
\hline \multirow[b]{2}{*}{$\begin{array}{l}\text { Benthic foraminifers from the } \\
\text { Claremont Shale and Oursan } \\
\text { Sandstone in Block IX }\end{array}$} & \multicolumn{4}{|c|}{ Claremont Shale } & \multicolumn{3}{|c|}{ Oursan Ss. } \\
\hline & 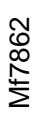 & 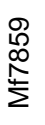 & 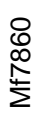 & $\begin{array}{l}\overline{6} \\
\infty \\
1 \\
\sum\end{array}$ & $\begin{array}{l}\frac{1}{6} \\
\frac{10}{10} \\
\frac{ \pm}{2}\end{array}$ & 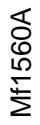 & 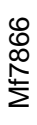 \\
\hline Bolivina advena ornata & $x$ & - & $x$ & - & - & - & - \\
\hline Bolivina salinasensis & - & - & - & - & - & $x$ & - \\
\hline Bolivina advena striatella & $x$ & $x$ & $x$ & $x$ & - & $x$ & $x$ \\
\hline Bolivina spp. & - & - & - & - & $x$ & - & - \\
\hline Buccella mansfieldi oregonensis & - & - & - & - & - & - & $x$ \\
\hline Buliminella curta & - & - & - & - & - & - & $x$ \\
\hline Buliminella elegantissima & - & - & - & - & - & - & - \\
\hline Buliminella subfusiformis & $x$ & $x$ & $x$ & - & - & - & $x$ \\
\hline Cassidulina panzana & - & $x$ & - & - & - & - & $x$ \\
\hline Cibicides fletcheri & - & - & - & - & - & - & - \\
\hline Elphidium spp. & - & - & $x$ & - & - & - & - \\
\hline Epistominella pacifica & - & - & - & - & - & $x$ & - \\
\hline Epistominella subperuviana & - & - & $x$ & - & - & - & - \\
\hline Fursenkoina californiensis & - & - & - & - & - & - & $x$ \\
\hline Fursenkoina spp. & - & - & - & - & - & - & - \\
\hline Haplophragmoides spp. & - & - & - & - & $x$ & - & - \\
\hline Karreriella spp. & - & $x$ & - & - & - & - & - \\
\hline Lenticulina spp. & - & - & $x$ & - & - & - & - \\
\hline Nonionella costifera & $x$ & $x$ & $x$ & $x$ & - & $x$ & $x$ \\
\hline Nonionella miocenica & $x$ & $x$ & $x$ & - & - & - & - \\
\hline Praeglobobulimina pupoides & - & $x$ & - & - & - & - & - \\
\hline Pullenia miocenica & - & - & - & - & - & $x$ & - \\
\hline Siphogenerina spp. & - & - & - & - & $x$ & $x$ & - \\
\hline Trifarina spp. & - & - & - & - & - & - & - \\
\hline Uvigerinella spp. & - & - & - & - & - & - & - \\
\hline Valvulineria californica s.s. & - & - & $x$ & - & - & - & - \\
\hline Valvulineria spp. & - & - & - & - & - & - & - \\
\hline
\end{tabular}

\section{Blocks VIII, X, and XI}

No new microfossil samples were examined from Blocks VIII, X, and XI. These blocks correspond to portions of the Livermore block of Buising and Walker (1995) and portions of the Diablo block of Graham and others (1984). Neogene units that overlie the Cretaceous Great Valley sequence in Block VIII include the Tice Shale, Briones Sandstone, an unnamed sandstone unit (Tss) of Graymer and others (1996), an unnamed freshwater limestone unit (Tlp) of Graymer and others (1996), and the Livermore Gravel. These formations range in age from late Miocene through Pleistocene (Graymer and others, 1996). Samples from the Oursan Sandstone (Mf7868) and Briones Sandstone (Mf8507, Mf8509, and Mf1662) of Block VIII are barren of foraminifers. In Block X, the Neogene unconformably overlies Franciscan Complex mélange. The Neogene units include the Briones and Neroly sandstones, which are considered late Miocene in age (Graymer and others, 1996). Samples from Block X include one from the Neroly Sandstone that is barren (Mf8508) and one from the Oursan Sandstone (Mf1215). The latter sample was reported to contain late Miocene or older microfossils that probably represented the Mohnian Stage. 
In Block XI, the Upper Cretaceous (?) to Eocene Tesla Formation unconformably overlies the Great Valley sequence and is in turn overlain by the Neogene Neroly and Cierbo Sandstones and Oro Loma Formation. Megafossils from the Tesla Formation suggest that it ranges from Late Cretaceous (late Maastrichtian Stage) or early Paleocene through the early middle Eocene Domengine megafossil stage (Throckmorton, 1988a, b). The Cierbo Sandstone unconformably overlies the Tesla Formation and contains upper Miocene megafossils (Huey, 1948; Throckmorton, 1988a, b). The overlying Neroly Sandstone contains vertebrate fossils that are assigned to the late Miocene Clarendonian NALM stage (C.A. Repenning in Throckmorton, 1988a).

\section{Summary of Tertiary Stratigraphy}

\section{Paleocene}

Paleocene strata in the eastern San Francisco Bay region, with the exception of the Tesla Formation in Block XI and unit Ta in Block I, rest unconformably on the Cretaceous Great Valley sequence. The age of the base of the Tesla Formation is in question and may be as old as Late Cretaceous or early Paleocene (Throckmorton, 1988a, b). Unit Ta is fault bounded and its stratigraphic relationship with Cretaceous strata is unknown.

Late Paleocene fossils occur in unit Ta in Block I, unit Tmz in Block VI, Tesla Formation in Block XI, Vine Hill Sandstone in Block IV, and unit Tps in Block VII (fig. 17, plate 1). No microfossils are present in the Tesla Formation in Block XI or in unit Ta in Block I, and the Paleocene age is based on megafossils. Microfossiliferous strata in units Tmz, Tps, and the Vine Hill Sandstone are assigned to the Ynezian Stage. Calcareous nannoplankton refine the age assignments for unit Tmz to zone CP4 (coeval with the early Ynezian Stage), for the Vine Hill Sandstone to zone CP7 (coeval with the middle Ynezian Stage), and forunit Tps to zone CP8 (coeval with the late Ynezian Stage) (Bukry and others, 1998). Although these formations are lithologically similar and represent a bathymetric continuum from east to west from upper bathyal $(15-500 \mathrm{~m})$ to middle bathyal $(500-1,500 \mathrm{~m})$ to lower bathyal $(2,000-4,000 \mathrm{~m})$, the age interpretations suggest that they represent discrete intervals within a time span of approximately 4 million years. 


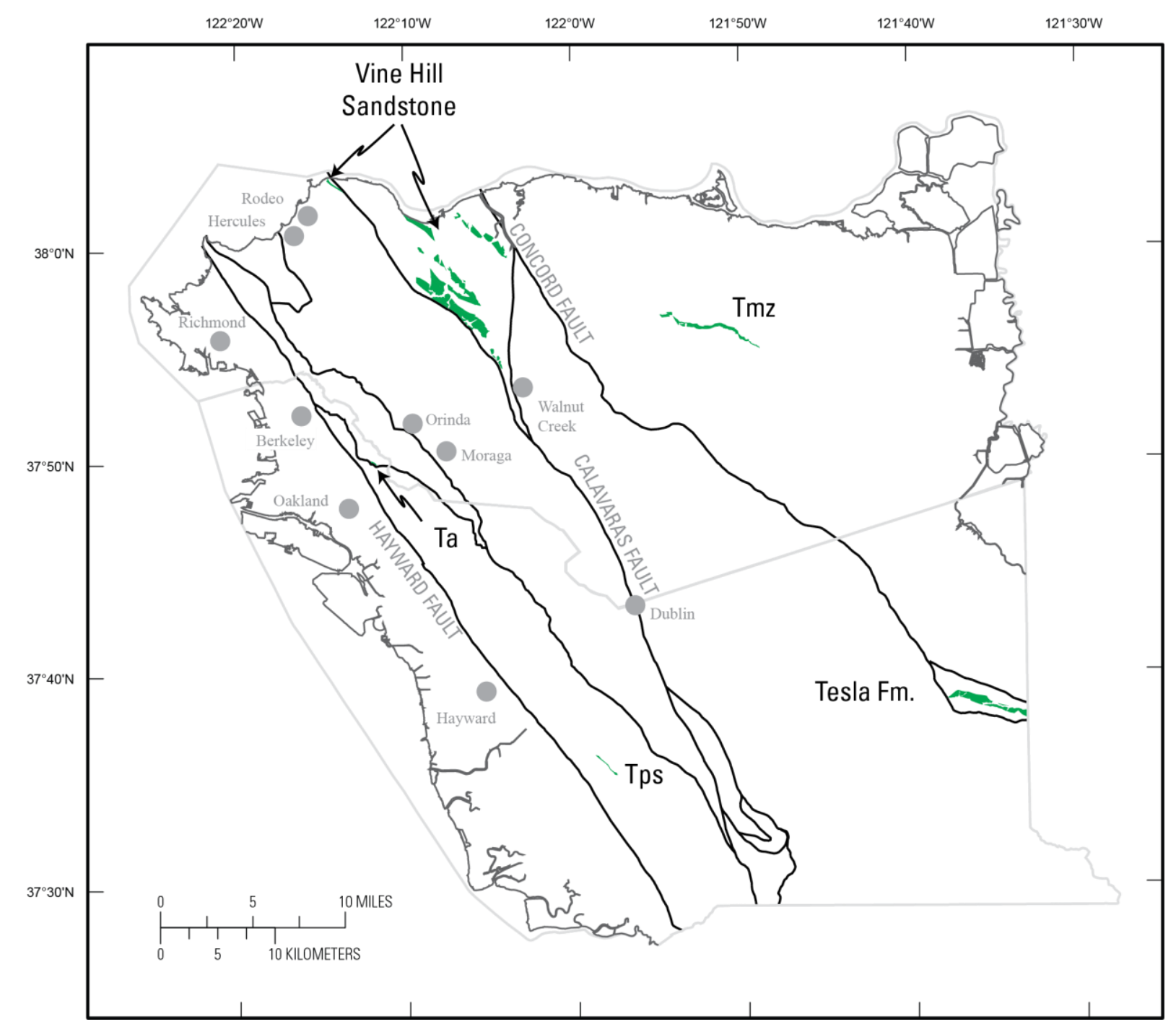

Figure 17. Map showing outcrop distribution of Paleocene strata (green) in Alameda and Contra Costa Counties.

\section{Eocene}

Eocene strata in the eastern San Francisco Bay region include the unnamed units Tshc, Tes, Tsh, and Tgs of Graymer and others (1994a) in Blocks I and II, the Meganos Formation in Block VI, Domengine Formation in Blocks V and VI, Kreyenhagen Formation in Block VI, Las Juntas Shale in Block IV, Muir Sandstone in Block IV, Escobar Sandstone in Block IV, Alhambra Formation in Block IV, Tolman Formation in Block VII, and questionably the upper part of the Tesla Formation in Block XI (fig. 18, plate 1). There are no microfossil data from the Tesla or Tolman formations. The Eocene formations rest unconformably on Cretaceous or Paleocene units, except for the units in Block I, which are mapped as faulted onto the Cretaceous. Three samples from unit Tsm, Block 1 of Graymer and others (1996) contain Eocene assemblages: one contains only long-ranging nondiagnostic arenaceous foraminifers; one contains only calcareous nannoplankton, is adjacent to a fault, and may be from the Tes unit; and the third sample contains late Eocene, Refugian Stage assemblages. Only this third sample seems give an indication of the oldest age for the Tsm unit-younger than the other Eocene units in the San Francisco Bay area. 


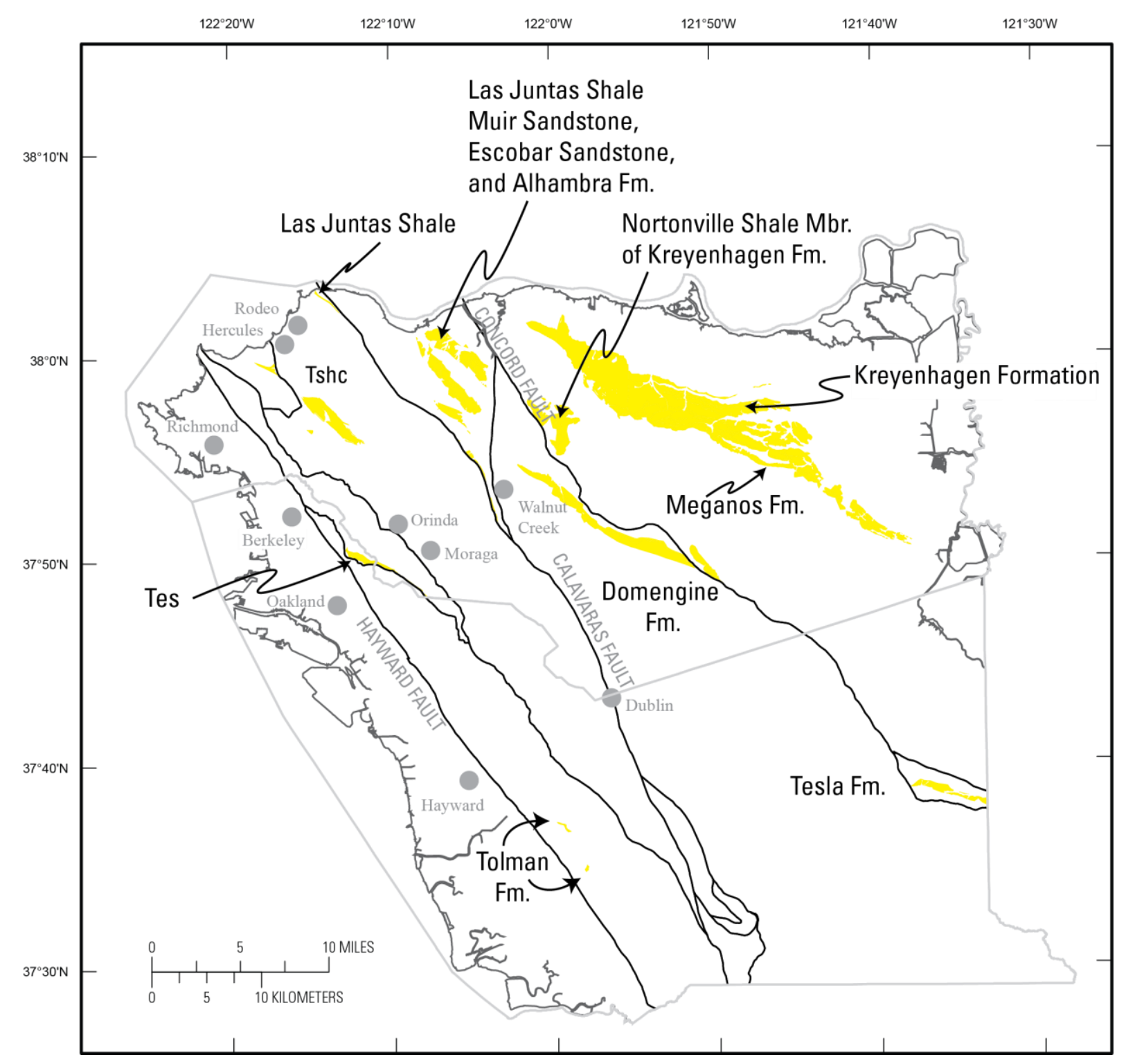

Figure 18. Map showing outcrop distribution of Eocene strata (yellow) in Alameda and Contra Costa Counties.

The oldest micropaleontologic samples are from unit Tes in Block I and the Meganos Formation in Block VI. Samples from unit Tes and the middle member (Tmc) of the Meganos Formation are early Eocene in age, and assigned to the early Penutian Stage and calcareous nannoplankton zone CP9b (Bukry and others, 1998). The lithology and microfossils in unit Tes indicate that deposition occurred at lower bathyal to abyssal depths and that this unit is similar to mottled mudstone units found throughout California, in particular unit Tgs of the Coyote Block near San Jose, the "mottled mudstones of Mt. Chual" from the Sierra Azul Block, also near San Jose, and the Bolado Park Formation near Tres Pinos (Graymer and others, 2002b; Kaar, 1962; Wentworth and others, 1999; McDougall, unpub. data). The middle member of the Meganos Formation was deposited at slightly shallower depths (middle to lower bathyal, 500-2,000 m), and the lithology is described as dark gray mudstone and siltstone, therefore representing a more nearshore facies. 
Both the upper part of unit Tes and the upper member (Tme) of the Meganos Formation are late early Eocene in age and assigned to the late Penutian and/or early Ulatisian Stages and calcareous nannofossil zone CP11 (Bukry and others, 1998). The late early Eocene age and assignment to zone CP11 is similar to the ages assigned to Tshc of Blocks II and III, the Las Juntas Shale in Block IV, and the Domengine Formation in Blocks V and VII. Except for the Las Juntas Shale, these units range in age from late early to early middle Eocene and are assigned to the late Penutian or the early Ulatisian Stages, calcareous nannoplankton zone CP11 and (or) CP12. The Las Juntas Shale begins in the early Eocene Penutian Stage, calcareous nannoplankton zone CP10. Deposition of these units occurred at lower middle to lower bathyal depths (1,500-2,000 m).

The Domengine Formation, Muir Sandstone, and Alhambra Formation extend into the middle Eocene Ulatisian Stage, calcareous nannoplankton zone CP13. The Escobar Sandstone does not contain microfossils. The overlying members of the Kreyenhagen Formation (Nortonville Shale, Markley Sandstone, and Sidney Flat Shale Members) occur in Block VI only. They are also middle Eocene in age and assigned to zones CP13 and CP14. Benthic foraminiferal faunas favor a slightly younger age, late middle to late Eocene, Narizian Stage. Bathymetry generally decreases with age: the younger formations were deposited at shallower depths than the older formations. In California, a widespread regression at the end of the Eocene and throughout the Oligocene is represented by unconformities in the eastern San Francisco Bay region.

\section{Oligocene}

Oligocene strata include the Kirker Tuff in Block VI and an unnamed unit, Tsm, in Block I (fig. 19, plate 1). No samples were examined from the Kirker Tuff. A single sample from unit Tsm yields an Oligocene, Zemorrian Stage, assemblage and suggests that deposition occurred at upper middle bathyal depths (500-1,500 m). This assemblage differs from the younger samples (Saucesian Stage, early Miocene) in unit Tsm.

\section{Miocene}

Miocene strata in the eastern San Francisco Bay region are composed of several major groups as well as individual formations (figs. 19, 20, 21, plate 1). Individual formations include units Tsm and Tush in Block I, the San Ramon Sandstone in Blocks II and IV, unit Tts in Block II, unit Tsh in Block VII, and the Temblor Formation in Block IX (fig. 19). The Monterey Group includes six alternating and interfingering sandstone and siliceous shale formations (in ascending order): Sobrante Sandstone, Claremont Shale, Oursan Sandstone, Tice Shale, Hambre Sandstone, and Rodeo Shale (fig. 20). The San Pablo Group, which overlies the Monterey Group, consists of (in ascending order) the Briones, Cierbo, and Neroly Sandstones, which are in turn overlain by the nonmarine Contra Costa Group (Orinda, Mulholland, Grizzly Peak, Siesta, and Bald Peak Formations) and other nonmarine formations and tuffs (Green Valley, Tassajara, and Tulare Formations, Lawlor Tuff, Oro Loma Formation, and unnamed units of Graymer and others, 1994, 1996) (fig. 21). 


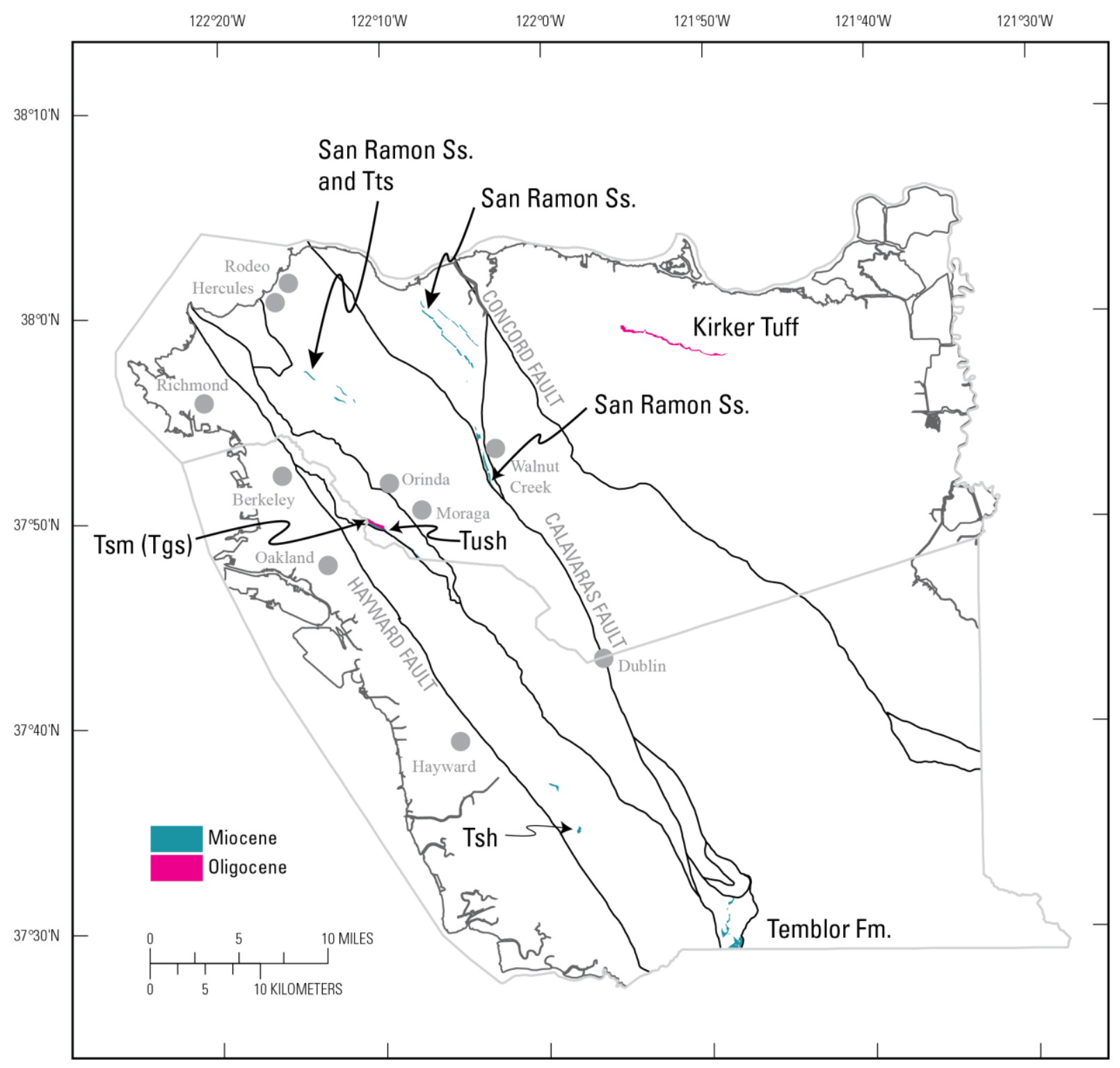

Figure 19. Map showing outcrop distribution of Oligocene (pink) and Miocene (teal) strata in Alameda and Contra Costa Counties, not including the Monterey Group. 


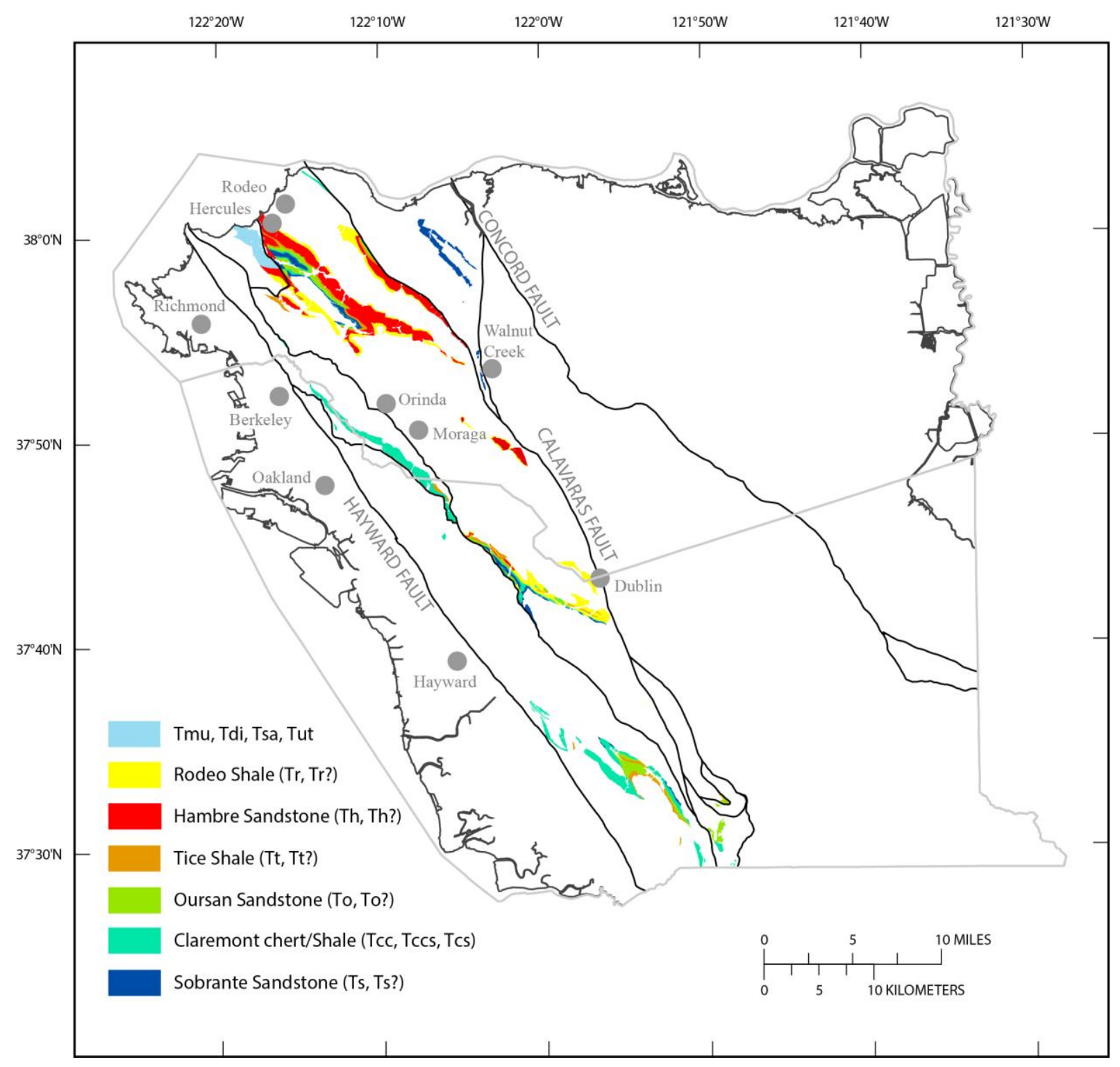

Figure 20. Map showing outcrop distribution of units comprising the Miocene Monterey Group in Alameda and Contra Costa Counties. 


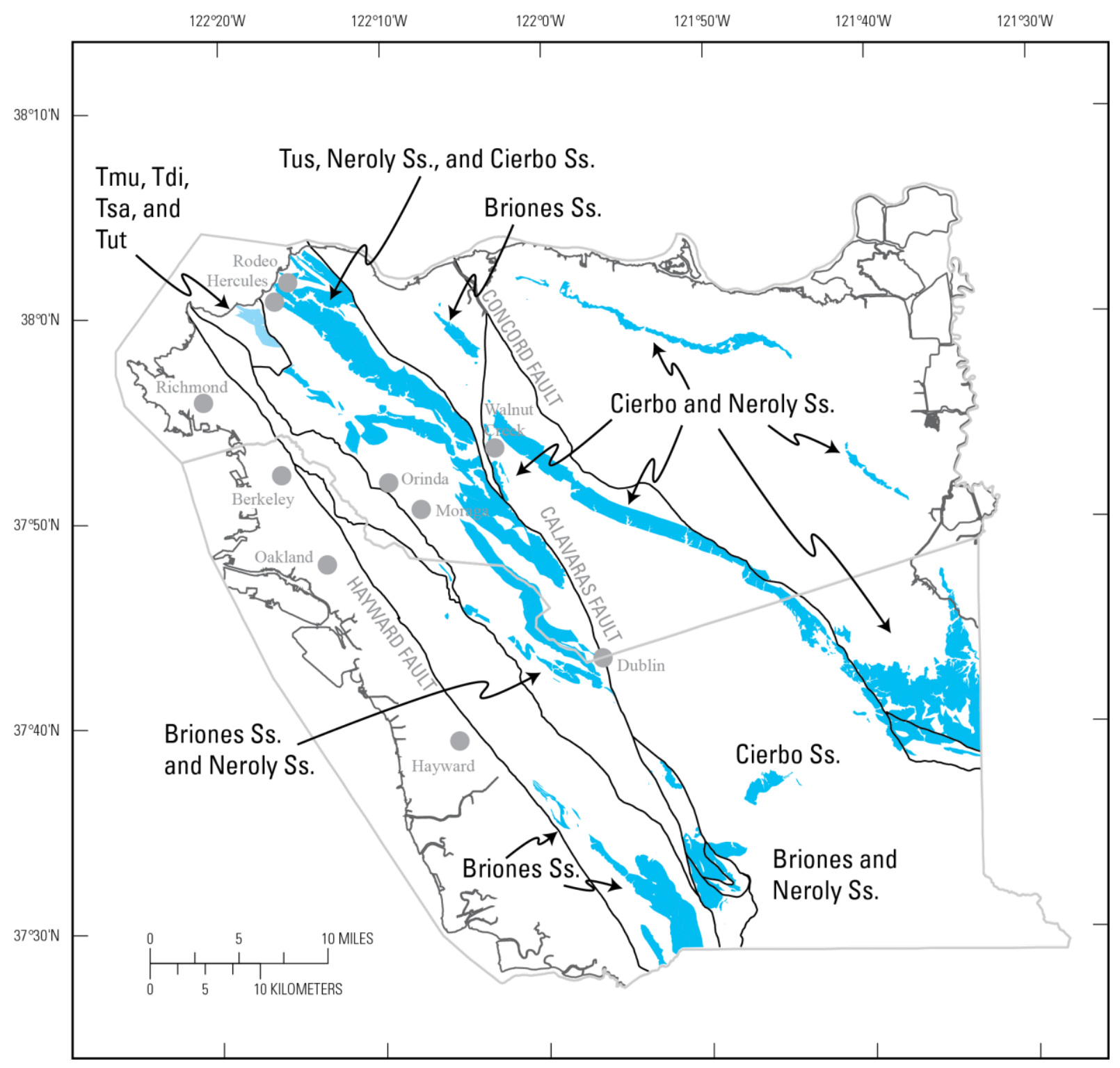

Figure 21. Figure 21. Map showing distribution of middle and upper Miocene marine strata (blue areas) in Contra Costa and Alameda Counties, not including the Monterey Group. 
The lower Miocene sediments rest unconformably on the Cretaceous or Paleogene sediments or are fault bounded below. No samples were examined from unit Tush, unit Tts, the San Ramon Sandstone, and the Temblor Formation, which are probably early Miocene in age. Only limited samples were examined from units Tsm and Tsh. The age of unit Tsm ranges from early Eocene to middle Miocene. The Eocene faunas are probably mislocated and are from other units. The single Zemorrian Stage assemblage may also be from another unit. The late Saucesian through Relizian Stage assemblages are well represented, in the same general area of Block I, and are assumed to represent the true age of unit Tsm. The majority of these assemblages indicate that deposition occurred at upper bathyal (150-500 m) or upper middle bathyal (500-1,500 m) depths. Lower bathyal depths are indicated for one sample only. The single sample examined from unit Tsh in Block VII also contains a Saucesian Stage fauna. Deposition of this unit occurred at upper middle bathyal depths (500-1,500 m).

The Monterey Group is present in most blocks, although lithology and internal stratigraphy varies considerably between blocks, and ranges in age from early Miocene (Saucesian Stage) to late middle Miocene (early Mohnian Stage). Samples from the Sobrante Sandstone are early Miocene and assigned to the Saucesian Stage based on microfossil samples (Block II) and stratigraphic position (Block VII), and to the Relizian Stage (early to early middle Miocene) based on scarce molluscan assemblages in Block IV (Schmidt, 1958). The Claremont Shale, which locally overlies the Sobrante Sandstone in Blocks II, VII, and IX, is middle Miocene in age and assigned to the Luisian Stage. The Oursan Sandstone, which overlies the Claremont Shale in Blocks II, VII, and IX, is also assigned to the middle Miocene Luisian Stage. A similar age is assumed for the Oursan Sandstone in Block VII despite the lack of samples. The Tice Shale, Hambre Sandstone, and Rodeo Shale in Block II are early middle Miocene in age and assigned to the Mohnian Stage. The Tice Shale was also sampled in Block VII and is assigned to the early Mohnian Stage. The Tice Shale in Blocks VIII and IX was not sampled but is assumed to represent the late middle Miocene.

Although some workers recognized the Monterey Group formations in Block III (Hill, 1983; Radbruch-Hall, 1969), Graymer and others (1994b) used informal names for the units (Tmu, Tsa, Tdi, Tut, and Tcgl). Microfossil samples from these units indicate that units Tmu and Tdi are middle Miocene in age and assigned to the Luisian Stage. Unit Tdi also contains benthic foraminifers that suggest this unit may range into the late middle to late Miocene Mohnian Stage. No microfossil samples were examined from the sandstones (unit Tsa and Tut) or the conglomerate (unit Tcgl), although tuff in unit Tcgl unit is late Miocene in age (6.1-5.7 Ma) and correlated with the Roblar Tuff (Graymer and others, 1994a). Deposition of unit Tmu occurred at upper middle bathyal depths (500-1,500 m), deposition of unit Tdi occurred at upper bathyal depths $(150-500 \mathrm{~m})$, and the Mohnian Stage sample in unit Tdi was deposited at upper middle bathyal depths (500-1,500 m). The faunal composition, age, and ecology of these units suggest correlation with the Claremont Shale for unit Tmu and with the Tice Shale forunit Tdi. Correlation of the sandstones is also indicated by stratigraphic position: unit Tsa with the Oursan Sandstone and unit Tut with the Hambre Sandstone.

The coastal onlap curve of Johnson and others (2005) correlates well with the interpreted depositional depths of the Monterey Group. The upper bathyal faunas of the Sobrante Sandstone overlie the outer neritic faunas of the San Ramon Sandstone and underlie the upper middle bathyal faunas of the Claremont Shale. Deposition of the Claremont Shale and Oursan Sandstone at upper middle bathyal depths (500-1,500 m) was followed by deposition of the Tice Shale at upper bathyal depths (150-500 m) near the shelf edge (about $150 \mathrm{~m}$ ). The Hambre Sandstone and Rodeo Shale were deposited at outer neritic depths $(50-150 \mathrm{~m})$. These formations and the depth interpretations indicate a gradual decrease in water depth from the older to younger units of the Monterey Group. 
In most locations, the San Pablo Group unconformably overlies the Monterey Group (fig. 21, plate 1) and represents a different style of sedimentation (Graham and others, 1983). Microfossil samples from the San Pablo Group, which consists of the Briones, Cierbo, and Neroly Sandstones, contain only rare to few foraminifers, but those faunas suggest that the Briones Sandstone is Miocene in age and that it was deposited in low oxygen conditions at upper bathyal depths (150-500 m). Additional published data on the Briones Sandstone include two microfossil samples from Block IV that are late middle to late Miocene, Mohnian Stage (Schmidt, 1958), observation of vertebrate fossils from Block II

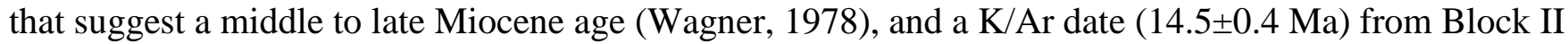
(Lindquist and Morganthaler, 1991) that suggests interfingering of the Briones Sandstone and the Monterey Group. However, the K/Ar sample is near the boundary between the Rodeo Shale, Hambre Sandtone, and Briones Sandstone, and based on the interpreted bathymetry of these formations, it is unlikely that they interfingered. The foraminiferal assemblage in the Cierbo Sandstone sample is longranging and indicates only a Miocene age. Deposition occurred at outer neritic depths near the shelf edge $(150 \mathrm{~m})$.

The overlying groups and formations in eastern San Francisco Bay region are nonmarine and not discussed in this study (plate 1).

\section{Acknowledgments}

The authors wish to thank the numerous geologists, paleontologists, and technicians for their assistance, discussions, and efforts to understand the San Francisco Bay area geology and paleontology. Special thanks to Sue Priest, Kristen Woody, Patricia Hall, Sarah Munn, and Elizabeth Mennow, who input and reviewed the data. We especially would like to thank Earl Brabb, Russ Graymer, Paul Stone, and Deborah Soltesz for reviewing this manuscript and providing insightful suggestions. 


\section{References Cited}

Addicott, W.O., 1970, Miocene gastropods and biostratigraphy of the Kern River area, California: U.S. Geological Survey Professional Paper 642, 174 p.

Addicott, W.O., 1973, Oligocene molluscan biostratigraphy and paleontology of the lower part of the Temblor Formation, California: U.S. Geological Survey Professional Paper 791, 48 p.

Addicott, W.O., Poore, R.Z., Barron, J.A., and McDougall, K., 1980, Miocene Biostratigraphy of the Indian Creek-Shell Creek area, Northern La Panza Range, San Luis Obispo County, California in Blake, G.H., ed., Neogene Biostratigraphy of the Northern La Panza Range, San Luis Obispo County, California: Los Angeles, Calif., Pacific Section, Society of Economic Paleontologists and Mineralogists, p. 11-37.

Almgren, A.A., Filewicz, M.V., and Heitman, H.L., 1988, Lower Tertiary foraminiferal and calcareous nannofossil zonation of California - an overview and recommendation in Filewicz, M.V., and Squires, R.L., eds., Paleogene stratigraphy, west coast of North America: Los Angeles, Calif., Volume and Guidebook, v. 58, Pacific Section, Society of Economic Paleontologists and Mineralogists, p. 83106.

Bailey, E.H., Irwin, W.P., and Jones, D.L., 1964, Franciscan and related rocks and their significance in the geology of western California: California Division of Mines and Geology Bulletin, v. 183, 177 p.

Bandy, O.L., 1949, Eocene and Oligocene Foraminifera from Little Stave Creek, Clarke County, Alabama: Bulletins of American Paleontology, v. 32, p. 1-153.

Bandy, O.L., 1961, Distribution of foraminifera, radiolaria and diatoms in sediments of the Gulf of California: Micropaleontology, v. 7, no. 1, p. 1-26.

Bandy, O.L., and Arnal, R.E., 1969, Middle Tertiary basin development, San Joaquin Valley, California: Geological Society of America Bulletin, v. 80, no. 5, p. 783-819.

Barr, F.T., and Berggren, W.A., 1980, Lower Tertiary biostratigraphy and tectonics of northwestern Libya in Salem, M.J. and Busrewil, M.T., eds., The geology of Libya, v. 2: New York, Academic Press, Inc., p. 163-192.

Bartow, J.A., compiler, 1992, Paleogene and Neogene time scales for southern California: U.S. Geological Survey, Open-File Report 92-212, 2 oversized sheets.

Berggren, W.A., 1972, A Cenozoic time-scale_-some implications for regional geology and paleobiogeography: Lethaia, v. 5, p. 195-215.

Berggren, W.A., Kent, D.V., Swisher, C.C., III, and Aubry, M.-P., 1995, A revised Cenozoic geochronology and chronostratigraphy in Berggren, W.A., Kent, D.V., Aubry, M.-P. and Hardenbol, J., eds., Geochronology, timescales and global stratigraphic correlation: Society for Sedimentary Geology, Special Publication, v. 54, p. 129-212.

Berggren, W.A., and Aubert, J., 1976, Late Paleogene (late Eocene and Oligocene) benthonic foraminiferal biostratigraphy and paleobathymetry of Rockall and Hatton-Rockall Basin: Micropaleontology, v. 22, no. 3, p. 307-326.

Berggren, W.A., and Aubert, J., 1983, Paleogene benthic foraminiferal biostratigraphy and paleobathymetry of the central Ranges of California, in Brabb, E.E., ed., Studies in Tertiary stratigraphy of the California Coast Ranges: U.S. Geological Survey Professional Paper 1213, p. 421.

Berggren, W.A., Aubry, M.-P., van Fossen, M., Kent D.V., and Norris, R.D., 2000, Integrated Paleocene calcareous plankton magnetobiochronology and stable isotope stratigraphy-DSDP site 384: Paleogeography, Paleoclimatology, Paleoecology, v. 159, no. 1-2, p. 1-51.

Berggren, W.A., Kent, D.V., Flynn, J.J., and van Couvering, J.A., 1985, Cenozoic geochronology: Geological Society of America Bulletin, v. 96, no. 11, p. 1407-1418. 
Berggren, W.A., and Miller, K.G., 1989, Cenozoic bathyal and abyssal calcareous benthic foraminiferal zonation: Micropaleontology, v. 35, no. 4, p. 308-320.

Berggren, W.A., and Pearson, P.N., 2005, A revised tropical to subtropical Paleogene planktonic foraminiferal zonation: Journal of Foraminiferal Research, v. 35, no. 4, p. 279-298.

Blake, G.H., 1981, Biostratigraphic relationship of Neogene benthic foraminifera from the Southern California Outer Continental Borderland to the Monterey Formation in Garrison, R.E., and Douglas, R.G., eds., The Monterey Formation and related siliceous rocks of California: Los Angeles, Calif., Pacific Section, Society Economic Paleontologists and Mineralogists, p. 1-14.

Blake, G.H., 1991, Review of the Neogene biostratigraphy and stratigraphy of the Los Angeles Basin and implications for basin evolution, in Biddle, K.T., ed., Active margin basins: Tulsa, Okla., American Association of Petroleum Geologists, p. 135-184.

Blake, M.C., Jr., Jones, D.L., Graymer, R.W., and Soule, A., 2000, Geologic map and map database of parts of Marin, San Francisco, Alameda, Contra Costa, and Sonoma counties, California: U.S. Geological Survey Miscellaneous Field Studies Map, MF-2337, 31 p., 1 sheet.

Blow, W.H., 1969, Late middle Eocene to recent planktonic foraminiferal biostratigraphy: Proceedings of the First International Conference on Planktonic Microfossils, Geneva, Switzerland, 1967, v. 1, p. 199-422.

Blow, W.H., 1979, The Cainozoic Globigerinida-A study of the morphology, taxonomy, evolutionary relationships and the stratigraphical distribution of some Globigerinida (mainly Globigerinacea): E.J. Brill, Leiden, Netherlands, 1,413 p.

Boersma, A., 1984, Handbook of common Tertiary Uvigerina: Microclimates Press, Stony Point, New York, 207 p.

Brabb, E.E., Jones, D.L., and Graymer, R.W., 2000, Geologic map and map database of the Palo Alto 30’ x 60' quadrangle, California: U.S. Geological Survey Miscellaneous Field Studies Map 2332, 28 p., 1 sheet.

Briggs, L.I., Jr., 1953, Geology of Ortigolita Peak quadrangle, California: California Division of Mines Bulletin 167, $61 \mathrm{p}$.

Buising, A.V., and Walker, J.P., 1995, Preliminary palinspastic paleogeographic reconstructions for the greater San Francisco Bay area, 15 Ma-5 Ma in Sangines, E.M., Andersen, D.W., and Buising, A.V., eds., Recent geologic studies in the San Francisco Bay area: Los Angeles, Calif., Society of Economic Paleontologists and Mineralogists, Pacific Section, v. 76, p. 141-160.

Bukry, D.,1973, Low-latitude coccolith biostratigraphic zonation in Edgar, N.T., and Saunders, J.B., eds., Initial reports of the Deep Sea Drilling Project: Washington, D.C., U.S. Government Printing Office, v. 15, p. 685-703.

Bukry, D., 1975, Coccolith and silicoflagellate stratigraphy, northwestern Pacific Ocean, Deep Sea Drilling Project Leg 32 in Larson, R.L., and Moberly, R., eds., Initial reports of the Deep Sea Drilling Project: Washington, D.C., U.S. Government Printing Office, v. 32, p. 677-701.

Bukry, D., Brabb, E.E., and Vedder, J.G., 1977, Correlation of Tertiary nannoplankton assemblages from the coast and peninsular ranges of California: Segundo Congreso Latinoamericano de Geologia, Memoria, T. 3, p. 1461-1483.

Bukry, D., 1991, Coccolith correlation of California Cenozoic geologic formations: U.S. Geological Survey Open-File Report 91-574, 30 p.

Bukry, D., Brabb, E.E., Powell, C.L., II, Jones, D.L., and Graymer, R.W., 1998, Recent Tertiary and Cretaceous nannoplankton collections from the San Francisco Bay region: U.S. Geological Survey Open-File Report 98-497, 33 p. 
Buzas, M.A., and Culver, S.J., 1990, Recent benthic foraminiferal provinces of the Pacific continental margin of North and Central America: Journal of Foraminiferal Research, v. 20, p. 326-335.

Case, J.E., 1968, Upper Cretaceous and lower Tertiary rocks, Berkeley and San Leandro Hills, California: U.S. Geological Survey Bulletin 1251-J, p. J1

Clark, B.L., 1921, Marine Tertiary of the West Coast of the United States: Journal of Geology, v. 29, p. 586-614.

Colburn, I.P., 1961, The tectonic history of Mount Diablo, California: Stanford, Calif., Stanford University, Ph.D. dissertation, 276 p.

Conduit, C., 1938, The San Pablo flora of west central California: Carnegie Inst. Washington Publ. Contr. Paleontology, v. 476, p. 217-268.

Culver, S.J., and Buzas, M.A., 1986, Distribution of recent benthic foraminifera off the North American Pacific Coast from California to Baja: Smithsonian Contributions to the Marine Sciences, $634 \mathrm{p}$.

Curtis, G.H., 1989, Berkeley Hills, in Wahrhaftig, C., ed., Geology of San Francisco and vicinity, 28th International Geological Congress field trip guidebook T105: Washington, D.C., American Geophysical Union, p. 47-52.

Cushman, J.A., 1922, The foraminifera of the Bryam calcareous marl at Byram, Mississippi in chap. E of Shorter contributions to general geology, 1921: U.S. Geological Survey Professional Paper 129, p. 87-122, 15 pls.

Cushman, J.A., 1951, Paleocene Foraminifera of the Gulf Coast Region of the United States and adjacent areas: U.S. Geological Survey Professional Paper 232, 75 p.

Donnelly, A.T., 1976, The Refugian Stage of California Tertiary-foraminiferal zonation, geologic history, and correlations with the Pacific Northwest: Santa Barbara, Calif., University of California, Ph.D. dissertation, $316 \mathrm{p}$.

Douglas, R.G., 1973, Benthonic foraminiferal biostratigraphy in the central North Pacific, Leg 17, Deep Sea Drilling Project, in Wintrer, E.L., Ewing, J.L., and others, eds., Initial Reports of the Deep Sea Drilling Project: Washington, D.C., U.S. Government Printing Office, p. 607-671.

Douglas, R.G., 1981, Paleoecology of continental margin basins - a modern case history from the borderland of southern California in Douglas, R.G., ed., Depositional systems of active continental margin basins, short course notes: Los Angeles, Calif., Pacific Section, Society of Economic Paleontologists and Mineralogists, p. 121-156.

Douglas, R.G., and Heitmann, H.L., 1979, Slope and basin benthic foraminifera of the California borderland in Doyle, L.J., and Pilkey, O.H., eds., Geology of continental slopes: Society of Economic Paleontologists and Mineralogists, Tulsa, Okla., p. 231-246.

Finger, K.L., 1990, Atlas of California Neogene foraminifera: Washington, D.C., Smithsonian Institution Cushman Foundation for Foraminiferal Research, Special Publication 28, 271 p., 128 pls.

Gibbard, P.L., Head, M.J., Walker, J.C., and the Subcommission on Quaternary Stratigraphy, 2010, Formal ratification of the Quaternary system/period and the Pleistocene series/epoch with a base at 2.58 Ma: Journal of Quaternary Science, v. 25, p. 96-102.

Goudkoff, P.P., 1945, Stratigraphic relations of Upper Cretaceous in Great Valley, California: Bulletin of the American Association of Petroleum Geologists, v. 29, p. 956-1007.

Gradstein, F.M., Kaminski, M.A., and Berggren, W. A., 1988, Cenozoic foraminiferal biostratigraphy of the central North Sea in Rogl, F. and Gradstein, F.M., eds., Workshop on agglutinated foraminifera, Vienna, 1986: Abhandlungen der Geologischen Bundesanstalt, Bd. 41, p. 97-108.

Gradstein, F.M., and Ogg, J.G., 2005, Time scale, in Selley, R.C., Cocks, L.R.M., and Plimer, I.R., eds., Encyclopedia of geology, v. 5: Oxford, United Kingdom, Elsevier Academic Press, p. 503-520. 
Gradstein, F.M., Ogg, J.G., and Smith, A.G., eds., 2004, A geologic time scale 2004: Cambridge, United Kingdom, Cambridge University Press, 589 p.

Graham, S.A., Gavigan, C., McCloy, C., Hitzman, M., Ward, R., and Turner, R., 1983, Basin evolution during the change from convergent to transform continental margin — an example from the Neogene of central California, in Cherven, V.B., and Graham, S.A., eds., Geology and sedimentology of the southwestern Sacramento Basin and East Bay Hills: Los Angeles, Calif., Pacific Section, Society of Economic Paleontologists and Mineralogists, Field Trip Guidebook, p. 101-117.

Graham, S.A., McCloy, C., Hitzman, M., Ward, R., and Turner, R., 1984, Basin evolution during the change from convergent to transform continental margin: American Association of Petroleum Geologists Bulletin, v. 68, no. 3, p. 233-249.

Graymer, R.W., 2000, Geologic map and map database of the Oakland Metropolitan Area, Alameda, Contra Costa, and San Francisco Counties, California: U.S. Geological Survey Miscellaneous Field Studies Map, MF-2342, 31 p., 1 sheet.

Graymer, R.W., Jones, D.L., and Brabb, E.E., 1994a, Preliminary geologic map emphasizing bedrock formations in Contra Costa County, California - a digital database: U.S. Geological Survey Open-File Report 94-622, 19 p.

Graymer, R.W., Jones, D.L., and Brabb, E.E., 1994b, Preliminary geologic map of the Niles 7.5-minute Quadrangle, Alameda County, California: U.S. Geological Survey Open-File Report 94-132, 1 sheet. Graymer, R.W., Jones, D.L., and Brabb, E.E., 1996, Preliminary geologic map emphasizing bedrock formations in Alameda County, California - a digital database: U.S. Geological Survey Open-File Report 96-252, 33 p.

Graymer, R.W., Jones, D.L., and Brabb, E.E., 2002a, Geologic map and map database of northeastern San Francisco Bay region, California, [including] most of Solano County and parts of Napa, Marin, Contra Costa, San Joaquin, Sacramento, Yolo, and Sonoma Counties: U.S. Geological Survey Miscellaneous Field Studies Map MF-2403, 30 pp., 1 sheet.

Graymer, R.W., Ponce, D.A., Jachens, R.C., Simpson, R.W., Phelps, G.A., and Wentworth, C.M., 2005, Three-dimensional geologic map of the Hayward Fault, northern California-correlation of rock units with variations in seismicity, creep rate, and fault dip: Geology, v. 33, p. 521-524.

Graymer, R.W., Sarna-Wojcicki, A.M., Walker, J.P., McLaughlin, R.J., and Fleck, R.J., 2002b, Controls on timing and amount of right-lateral offset on the East Bay fault system, San Francisco Bay region, California: Geological Society of America Bulletin, v. 114, p. 1471-1479.

Hall, C.A.J., 1958, Geology and paleontology of the Pleasanton Area, Alameda and Contra Costa counties, California: University of California Publications in Geological Sciences, v. 34, no. 1, 63 p.

Haller, C.R., 1980, Pliocene biostratigraphy of California in Kleinpell, R.M., ed., The Miocene stratigraphy of California revisited, also Pliocene biostratigraphy of California: Tulsa, Okla., American Association of Petroleum Geologists, p. 183-341.

Ham, C.K., 1952, Geology of the Las Trampas Ridge, Berkeley Hills, California: California Division of Mines and Geology Special Report 22, 22 p.

Haq, B.U., Hardenbol, J., and Vail, P.R., 1987a, Chronology of fluctuating sea levels since the Triassic: Science, v. 235, p. 1156-1166.

Haq, B.U., Hardenbol, J., and Vail, P.R., 1987b, The new chronostratigraphic basis of Cenozoic and Mesozoic sea level cycles in Ross, C.A., and Haman, D., eds., Timing and depositional history of eustatic sequences - constraints on seismic stratigraphy: Washington, D.C., Smithsonian Institution Cushman Foundation for Foraminiferal Research, Special Publication 24, p. 7-13. 
Haq, B.U., Hardenbol, J., and Vail, P.R., 1988, Mesozoic and Cenozoic chronostratigraphy and cycles of sea-level change in Wilgus, C.K., Hastings, B.S., Ross, C.a., Posamentier, H., van Wagoner, J., and Kendall, C.G.S.C., eds., Sea-level changes-An integrated approach: Society of Economic Paleontologists and Mineralogists, Special Publication 42, p. 71-108.

Helley, E.J., and Graymer, R.W., 1997a, Quaternary geology of Alameda County, and parts of Contra Costa, Santa Clara, San Mateo, San Francisco, Stanislaus, and San Joaquin Counties, California-a digital database: U.S. Geological Survey Open-File report 97-97, 13 p.

Helley, E.J., and Graymer, R.W., 1997b, Quaternary geology of Contra Costa County, and surrounding parts of Alameda, Marin, Sonoma, Solano, Sacramento, and San Joaquin counties, California-a digital database: U.S. Geological Survey Open-File report 97-98, 9 p., 1 sheet.

Hill, J.M., 1983, Stratigraphy of the Monterey rocks in the east San Francisco Bay Area, California, in Cherven, V.B., and Graham, S.A., eds., Geology and sedimentology of the southwestern Sacramento Basin and East Bay Hills: Los Angeles, Calif., Society of Economic Paleontologists and Mineralogists, Pacific Section, Field Trip Guidebook, p. 119-124.

Huey, A.S., 1948, Geology of the Tesla quadrangle, California: California Division of Mines and Geology Bulletin, v. 140, 75 p.

Ingle, J.C., Jr., 1980, Cenozoic paleobathymetry and depositional history of selected sequences within the southern California Borderland: Washington, D.C., Smithsonian Institution Cushman Foundation for Foraminiferal Research, Special Publication 19, p. 163-195.

Ingle, J. C., Jr., and Keller, G., 1980, Benthic foraminiferal biofacies of the Eastern Pacific Margin between $40^{\circ} \mathrm{S}$ and $32^{\circ} \mathrm{N}$ in Field, M.E., Douglas, R.G. and others, eds., Quaternary depositional environments of the Pacific Coast: Los Angeles, Calif., Pacific Section, Society of Economic Paleontologists and Mineralogists, p. 341-355.

Johnson, C.L., Bloch, R.B., and Graham, S.A., 2005, Tertiary sequences of the central San Joaquin Basin, California-Age control and eustatic versus tectonic forcing factors: Los Angeles, Calif., American Association of Petroleum Geologists, Pacific Section, MP49, 1 sheet.

Jones, D.L., and Curtis, G.H., 1991, Guide to the geology of the Berkeley Hills, central Coast ranges, California, in Sloan, D., and Wagner, D.L., eds., Geologic excursions in northern California-San Francisco to the Sierra Nevada: California Divisions of Mines and Geology, Special Publication, p. 63-74.

Kaar, R.F., 1962, Lower Tertiary foraminifera from north central San Benito County, California: Berkeley, University of California, M.S. thesis, 148 p.

Kaminski, M.A., Gradstein, F.M., and Beggren, W.A., 1988, Fysch-type agglutinated foraminiferal assemblages from Trinidad-Taxonomy, stratigraphy and paleobathymetry in Rogl, F. and Gradstein, F.M., eds., Workshop on agglutinated foraminifera Vienna, 1986: Abhandlungen der Geologischen Bundesanstalt, Bd. 41, p. 155-227.

Kaminski, M.A., and Gradstein, F.M., 2005, Atlas of Paleogene cosmopolitan deep-water agglutinated foraminifera: Krakow, Poland, Grzybowski Foundation, Special Publication 10, 547 p.

Katz, M.E., and Miller, K.G., 1991, Early Paleogene benthic foraminiferal assemblage and stable isotope composition in the Southern Ocean, in Ciesielski, P.F., Kristofferson, Y., and others, eds., Proceedings of the Ocean Drilling Program, Scientific Results, Subantarctic south Atlantic: College Station, Texas, Ocean Drilling Program, v. 114, p. 481-512.

King, C., 1989, Cenozoic of the North Sea, in Jenkins D.G. and Murray, J.W., eds., Stratigraphical Atlas of fossil foraminifera: British Micropaleontological Society Series, p. 418-489.

Kleinpell, R.M., 1938, Miocene stratigraphy of California: Tulsa, Okla., American Association of Petroleum Geologists, 450 p. 
Kleinpell, R.M., 1980, The Miocene stratigraphy of California revisited: American Association of Petroleum Geologists, Studies in Geology, v. 11, p. 1-182.

Laiming, B.L., 1939, Some foraminiferal correlation in the Eocene of San Joaquin Valley, California: University of California Press, Proceedings of the Sixth Pacific Science Congress of the Pacific Science Association, v. 2, p. 535-568.

Lankford, R.R., and Phleger, F.B., 1973, Foraminifera from the nearshore turbulent zone, western North America: Journal of Foraminiferal Research, v. 3, p. 101-132.

Lindquist, T.A., and Morganthaler, J.D., 1991, Radiometric ages of rocks in the San Francisco-San Jose quadrangles, California: California Division of Mines and Geology Map 5A, 4 sheets.

Loeblich, A.R., Jr, 1958, Danian Stage of Paleocene in California: American Association of Petroleum Geologists, Bulletin, v. 42, p. 2260-2261.

Mallory, V.S., 1959, Lower Tertiary biostratigraphy of the California Coast Ranges: America Association of Petroleum Geologists: Tulsa, Okla., American Association of Petroleum Geologists, $416 \mathrm{p}$.

Martini, E., 1970, Standard Paleogene calcareous nannoplankton zonation: Nature, v. 226, p. 560-561.

Martini, E., 1971, Standard Tertiary and Quaternary calcareous nannoplankton zonation in Farinacci, A., ed., Proceedings of the Second Plankton Conference: Edizioni Teconscienza, Rome, Italy, p. 739785.

McDougall, K., 1980, Paleoecological evaluation of late Eocene biostratigraphic zonations of the Pacific coast of North America: Society of Economic Paleontologists and Mineralogists, Paleontological Monograph, no. 2, 75 p.

McDougall, K., 1983, Upper Eocene to lower Miocene benthic foraminifers from the Santa Cruz Mountains area, California in Brabb, E.E., ed., Studies in Tertiary stratigraphy of the California Coast Ranges: U. S. Geological Survey Professional Paper 1213, p. 61-77.

McDougall, K., 1985, Miocene to Pleistocene benthic foraminifers from the Santa Cruz Mountains area, California in Brabb, E.E., ed., Studies in tertiary Stratigraphy of the California Coast Ranges: U.S. Geological Survey Professional Paper 1213, p. 61-77.

McDougall, K., 1988, Re-evaluation of early Eocene, Penutian Stage: American Association of Petroleum Geologists, Society of Economic Paleontologists and Mineralogists, and Society of Exploration Geophysics, Pacific Section Annual Meeting Program with Abstracts, abs. 91035.

McDougall, K., 1989, Paleogene benthic foraminifers from the Loma Prieta Quadrangle, California: U.S. Geological Survey, Open-File Report 89-649, 116 p.

McDougall, K., 1991, Benthic foraminifera from the Laurel Quadrangle, California: U.S. Geological Survey, Open-File Report 91-13, 66 p.

McDougall, K., 1993, Eocene benthic foraminiferal assemblages of the Palo Alto $71 \frac{2}{2}$ Quadrangle, California: U.S. Geological Survey, Open-file Report 93-180, 93 p.

McDougall, K., 1995, Age of the Fishcreekian Transgression: Paleos, v. 10, p. 199-220.

McDougall, K., 2008, California Cenozoic biostratigraphy_Paleogene, chap, 4 in Hosford Scheirer, A., ed., Petroleum systems and geologic assessment of oil and gas in the San Joaquin Basin Province, California: U.S. Geological Survey Professional Paper 1713, 30 p.

van Morkhoven, F.P.C.M., Berggren, W.A., and Edwards, A.S., 1986, Cenozoic cosmopolitan deepwater benthic foraminifera: Bulletin des Centres de Recherches Exploration-Production ElfAquitaine, Memoire, v. 11, 421 p.

Murray, J.W., 1991, Ecology and palaeoecology of benthic foraminifera: New York, Longman Scientific and Technical, 397 p. 
Natland, M.L., 1952, Pleistocene and Pliocene stratigraphy of southern California: Los Angeles, University of California at Los Angeles, Ph.D. dissertation.

Nilsen, T.H., and Brabb, E.E., 1979, Geology of the Santa Cruz Mountains, California: Geological Society of America Cordilleran Section field trip guidebook, no. 7, 97 p.

Okada, H., and Bukry, D., 1980, Supplemental modification and introduction of code numbers to the low-latitude coccolith biostratigraphic zonation (Bukry, 1973; 1975): Marine Micropaleontology, v. 5, p. 321-325.

Pelletier, W.J., 1951, Paleontology and stratigraphy of the Clarendonian continental beds west of Tracy, California: Berkeley, University of California, M.S. thesis, 91 p.

Pflum, C.E., and Frerichs, W.D., 1976, Gulf of Mexico deep-water foraminifera: Washington, D.C., Smithsonian Institute Cushman Foundation for Foraminiferal Research, Special Publication 14, 125 p.

Plummer, H.J., 1926, Foraminifera of the Midway Formation in Texas: University of Texas Bulletin, v. 2644, p. 3-206.

Poore, R. Z., 1976, Microfossil correlation of California lower Tertiary sections-a comparison: U.S. Geological Survey Professional Paper 743-F, 8 p., 2 pls.

Radbruch-Hall, D.H., 1957, Areal and engineering geology of the Oakland West quadrangle, California: U.S. Geological Survey Miscellaneous Investigation Series Map I-239.

Radbruch-Hall, D.H., 1969, Areal and engineering geology of the Oakland East quadrangle, California: U.S. Geological Survey Miscellaneous Investigation Series Map GQ-769.

Radbruch, D.H., and Case, J.E., 1967, Preliminary geologic map and engineering information, Oakland and vicinity, California: U.S. Geological Survey Open-File Report 67-183, scale 1:24,000, 3 pls.

Resig, J.M., 1981, Biogeography of benthic foraminifera of the northern Nazca plate and adjacent continental margin: Geological Society of America Memoir 154, p. 619-666.

Revets, S.A., 1991, The nature of Virgulinella Cushman, 1932, and the implications for its classification: Journal of Foraminiferal Research, v. 21, no. 4, p. 293-298.

Richley, K.A., 1948, Lower Pliocene horses from Black Hawk Ranch, Mount Diablo, California: University of California Publications in Geological Science, v. 28, p. 1-44.

Robinson, G.D., 1956, Geology of the Hayward Quadrangle, California: U.S. Geological Survey Geologic Quadrange 88, 1 sheet, scale 1:24,000.

Sarna-Wojcicki, A.M., 1976, Correlation of late Cenozoic tuffs in the central California Coast Ranges by means of trace and minor element chemistry: U.S. Geological Survey Professional Paper 972, 30 p. Sarna-Wojcicki, A.M.,1992, Long-term displacement rates of the San Andreas fault system in northern California from the 6-Ma Roblar tuff, in Borchardt, G., Hirschfeld, S.E., Lienkaemper, J.J., McCellan, P., Williams, P.L., and Wong, I.G., eds., Proceedings of the second conference on earthquake hazards in the eastern San Francisco Bay area: California Division of Mines and Geology Special Publication 113, p. 29-30.

Savage, D.E., 1951, Late Cenozoic vertebrates of the San Francisco Bay region: University of California Publications Bulletin of the Department of Geological Sciences, v. 28, p. 215-314.

Savage, D.E., 1955, Nonmarine lower Pliocene sediments in California - a geochronologic-stratigraphic classification: University of California Publications in Geological Sciences, v. 31, no. 10, 26 p.

Savage, D.E., and Barnes, L.G., 1972, Miocene vertebrate chronology of the west coast of North America, in Stinemeyer, E.H., ed., Proceedings of the Pacific Coast Miocene Symposium: Los Angeles, Calif., Society of Economic Paleontologists and Mineralogists, Pacific Section, p. 125-145. Schenck, H.G., and Kleinpell, R.M., 1936, Refugian Stage of Pacific Coast Tertiary: American Association of Petroleum Geology Bulletin, v. 20, p. 215-225. 
Schmidt, O.M., 1958, San Ramon Sandstone in the Pacheco syncline, California: Stanford, Calif., Stanford University, M.S. thesis, 53 p.

Sliter, W.V., 1968, Upper Cretaceous benthic foraminifera from southern California and northwestern Baja California, Mexico: The University of Kansas Paleontological Contributions, no. 49, Protozoa, art. 7, $141 \mathrm{p}$.

Sliter, W.V., McDougall, K., Murchey, B.L., and Kohnen, E.V., 1993, Mesozoic and Cenozoic microfossils from geologic units within the San Jose 1:100,000 quadrangle, California: U.S. Geological Survey Open-File Report 93-344, 119 p.

Smith, H.P., 1957, Lower Tertiary foraminifera from Contra Costa County, California: University of California Publications in Geological Sciences, v. 32, p. 127-207.

Smith, P.B., 1964, Ecology of benthonic species: U.S. Geological Survey Professional Paper, v. 429-B, $55 \mathrm{p}$.

Stirton, R.A., 1939, Cenozoic mammal remains from the San Francisco Bay region: University of California Publications in Geological Sciences, v. 24, no. 13, p. 339-409.

Throckmorton, C.K., 1988a, Depositional environments and molluscan biostratigraphy of the Tesla Formation, central California, in Filewicz, M.V., and Squires, R.L., eds., Paleogene Stratigraphy, west coast of North America: Los Angeles, Calif., Society of Economic Paleontologist and Mineralogists, Pacific Section, p. 209-223.

Throckmorton, C.K., 1988b, Geology and paleontology of the Tesla Formation, Alameda and San Joaquin Counties, central California: U.S. Geological Survey Open-File Report 88-59, p. 174-177.

Tjalsma, R.C., and Lohmann, G.P., 1983, Paleocene-Eocene bathyal and abyssal benthic foraminifer from the Atlantic Ocean: Micropaleontology Special Publication, v. 4, 90 p.

Wagner, J.R., 1978, Late Cenozoic history of the Coast Ranges east of San Francisco Bay: Berkeley, University of California, Ph.D. dissertation, $161 \mathrm{p}$.

Weaver, C.E., Beck, R.S., Bramlette, M.N., Carlson, S.A., Forrest, L.C., Kelley, F.R., Kleinpell, R.M., Putman, W.C., Taliaferro, N.L., Thorup, R.R., Ver Wiebe, W.A., Watson, E.A., 1944, Correlation of the marine Cenozoic formations of western North America [chart no. 11]: Geological Society of America Bulletin, v. 55, no. 5, p. 569-598.

Weaver, C.E., 1953, Eocene and Paleocene deposits at Martinez, California: University of Washington Publications in Geological Sciences, v. 7, 102 p.

Wentworth, C.M., 1993, General distribution of geologic materials in the southern San Francisco Bay Region, California - a digital map database: U.S. Geological Survey Open-File Report 93-693, 12 p, 1 sheet.

Wentworth, C.M., Blake, M.C., Jr., McLaughlin, R.J., and Graymer, R.W., 1999, Preliminary geologic map of the San Jose 30 x 60' quadrangle, California - a digital map image: U.S. Geological Survey Open-File Report 98-795, 47 p.

White, W.R., 1956, Pliocene and Miocene foraminifera from the Capistrano Formation, Orange County, California: Journal of Paleontology, v. 30, p. 237-260.

Wissler, S.G., 1943, Stratigraphic relations of the producing zone of the Los Angeles basin oil field: California Division of Oil and Gas Bulletin 118, p. 209-234.

Wissler, S.G., 1958, Correlation chart of producing zones of Los Angeles basin oil fields in Higgins, J.W., ed., A guide to the geology and oil fields of the Los Angeles and Ventura regions: American Association of Petroleum Geologists Pacific Section, p. 59-61.

Woodruff, F., 1985, Changes in Miocene deep-sea benthic foraminiferal distribution in the Pacific Ocean-relationship to paleoceanography in Kennett, J.P., ed., The Miocene oceanpaleoceanography and biogeography: Geological Society of America Memoir 163, p. 131. 


\section{Appendix-Taxonomic Notes}

The following section includes a list of benthic foraminiferal species identified in the course of this study. Specimens that could only be identified to genus are not included unless they are considered unique or of potential stratigraphic or environmental value. Species listed include the first describer of the species and a reference to a recent or thorough taxonomic listing for reference. Age or ecologic attributes of the species used in this study are given. Coeval planktic foraminiferal or calcareous nannoplankton zones are given in parenthesis.

Alabamina wilcoxensis Toulmin, 1941, Jour. Paleo., v. 15, p. 603, pl. 81, figs. 10-14; p. 605, tf. 4A-C. Range: latest Paleocene through late Eocene (P6b-P16) with questionable occurrences in the Paleocene (P4) (McDougall, 2008). Ecology: in middle and late Eocene restricted to depths $>2000 \mathrm{~m}$ (Tjalsma and Lohmann, 1983).

Allomorphina conica Cushman and Todd, 1949, Cushman Lab. Foram. Res., Contr., v. 25, p. 62, pl. 11, fig. 8a-c. Range: late Bulitian through early Ulatisian Stages (P6b to P9) (Mallory, 1959; McDougall, unpublished).

Allomorphina halli Jennings, 1936, Bull. Amer. Paleo., v. 23, no. 78, p. 34, pl. 4, fig. 5a,b. Allomorphina macrostoma Karrer, 1862, K. Akad. Wiss. Wien., Math.-Naturw. Cl., Sitzber., Osterreich, Bd. 44, Abth. 1, p. 448, pl. 2, fig. 4a-d. Ecology: outer neritic biofacies, 50-150 m (Ingle, 1980). Ammobaculites expansus Plummer, 1933, Texas Univ. Bull., no. 3201, p. 65, pl. 5, figs. 4-6. Ammobaculites sp. of Smith, 1957, p. 150, pl. 17, fig. 12a-b.

Ammobaculites paleocenicus Cushman, 1947, Cushman Lab. Foram. Res., Contr., v. 23, p. 77. Ammodiscus incertus (d'Orbigny)—Mallory, 1959, p. 108, pl. 1, figs. 11, 12; pl. 39, fig. 2.

Ammodiscus pennyi Cushman and Jarvis, 1928, Cushman Lab. Foram. Res., Contr., v. 4, p. 87, pl. 12, figs. 4-5. Range: Late Cretaceous (Maastrichtian) through late Paleocene (P6a) (Kaminiski and others, 1988). Ecology: bathyal to abyssal biofacies, $\geq 150 \mathrm{~m}$ (Kaminiski and Gradstein, 2005).

Amphimorphina becki Mallory, 1959, p. 215, pl. 19, fig. 1a,b. Range: late middle Eocene, restricted to the early Narizian Stage (Mallory, 1959).

Amphimorphina californica Cushman and McMasters, 1936, Jour. Paleo., v. 10, p. 513, pl. 16, figs. 3135. Range: Ulatisian through early Narizian stages, middle Eocene (P9-P12) (McDougall, 2008). Amphimorphina ignota Cushman and Siegfus, Cushman Lab. Foram. Res., Contr., v. 11, p. 27, pl. 6, figs. 10-13. Range: early Eocene through early Oligocene (P6b-P18) (Mallory, 1959; Poore, 1976; Tjalsma and Lohmann, 1983; McDougall, 2008).

Amphimorphina jenkinsi $($ Church $)=$ Plectofrondicularia jenkinsi Church, 1931, Calif. Dept. Nat. Div. Mines, v. 27, p. 208, pl. A, figs. 5, 7-9. Range: late Eocene, late Narizian Stage (Mallory, 1959). Anomalina madrugaensis Cushman and Bermudez, 1948, Cushman Lab. Foram. Res., Contr., v. 24, p. 86, pl. 15, figs. 4-6.

Anomalina garzaensis Cushman and Siegfus, 1939, Cushman Lab. Foram. Res., Contr., v. 11, p. 32, pl. 7, fig. 3a-c. Range: early Penutian through late Narizian Stages (P7- P10) (Mallory, 1959; Poore, 1976; McDougall, unpublished). Ecology: upper middle bathyal biofacies, 500-1500 m (Ingle, 1980).

Anomalina keenae Martin, 1943, Stanford Univ. Pub. Geol. Sci., v. 3, p. 28, pl. 7,fig, 5a-c. Anomalina regina Martin, 1943, Stanford Univ. Pub. Geol. Sci., v. 3, p. 28, pl. 9, fig. 3a-c. Range: Penutian through Ulatisian Stages, early to middle Eocene (P6b-P10) (McDougall, 1988; 2008). Ecology: UDL is in the upper middle bathyal biofacies, 500-1500 m (Ingle, 1980). 
Anomalina salinasensis Kleinpell, 1938, p. 347, pl. 13, fig. 1. Range: Saucesian through Luisian Stages (Finger, 1990; Kleinpell, 1938). Ecology: lower middle bathyal biofacies, 1,500-2,000 m (Ingle, 1980).

Anomalinoides acutus Plummer, 1926, Texas Univ. Bull., no. 2644, p. 149, pl. 10, fig. 2. Range: Ynezian through Penutian Stages, late Paleocene through early Eocene (P4-early P9) (Mallory, 1959; McDougall, 2008). Ecology: outer neritic biofacies, 50-150 m (Ingle, 1980).

Anomalinoides capitatus (Gumbel) — van Morkhoven and others, 1986, p. 276-278, figs. 1-2. Range: Eocene through early Oligocene with doubtful occurrences in early Oligocene (P6b-P18; P19P20) (van Morkhoven and others, 1986; McDougall, 2008). Ecology: lower bathyal biofacies, $\geq$ $2000 \mathrm{~m}$ (Ingle, 1980; van Morkhoven and others, 1986).

Anomalinoides midwayensis $($ Plummer) = Truncatulina midwayensis Plummer, 1926, Texas Univ. Bull., no. 2644, p. 141, pl. 9, fig. 7; pl. 15, fig. 3. Range: early Ynezian to late Bulitian Stages

(Mallory, 1959; McDougall, unpublished).

Anomalinoides praeacuta $($ Vasilenko $)=$ Anomalina praeacuta Valsilenko-Tjalsma and Lohman, 1983, p. 4, pl. 4, fig. 10; pl. 10, fig. 8a-b. Range: Paleocene through early Eocene (P1-P6b) (Tjalsma and Lohmann, 1983).

Anomalinoides rubiginosus (Cushman)—van Morkhoven and others, 1986, p. 366-368, pl. 119, figs.

1,2. Range: Late Cretaceous through Paleocene (P5) (van Morkhoven and others, 1986).

Ecology: middle neritic to bathyal biofacies, 50->2000 m (van Morkhoven and others, 1986).

Anomalinoides semicribratus (Beckman) - van Morkhoven and others, 1986, p. 147-148, pl. 48, figs. 1-

3. Range: middle Eocene (P12 and younger) through middle Miocene (N12); transitional forms can be found in the middle Eocene (P9-P11) and in the late middle Miocene (N13-N14)

(Tjalsma and Lohmann, 1983; van Morkhoven and others, 1986).

Anomalinoides welleri $($ Plummer) = Truncatulina welleri Plummer, 1926, Texas Univ. Bull., no. 2644, p. 143 , pl. 9 , fig. 6 .

Aragonia aragonensis (Nuttall) — van Morkhoven and others, 1986, p. 308-314, pl. 101A, figs. 1-3; pl. 101B, figs. 1-4; pl. 101C, figs. 103. late Paleocene, late Ynezian Stage through latest middle Eocene, early Narizian Stage (P5-P14) (Mallory, 1959; Tjalsma and Lohmann, 1983; van Morkhoven and others, 1986). Ecology: upper bathyal biofacies, 150-500 m (Ingle, 1980); A. aragonensis is considered primarily a lower bathyal and abyssal form (van Morkhoven and others, 1986).

Astacolus crepidulus $($ Fitchel and Moll $)=$ Nautilis crepedulus Fitchel and Moll, 1798, Testacea Microscopia, p. 107, th. 19, figs. g-i.

Astigerina simiensis Cushman and McMasters, 1936, Jour. Paleo., v. 10, p. 515, pl. 77, figs. 1-4. Baggina californica Cushman, 1926, Cushman Lab. Foram. Res., Contr., v. 2, p. 64, pl. 9, fig. 8a-c. Range: Zemorrian through Delmontian Stages (Finger, 1990; Kleinpell, 1938). Ecology: transitional between outer neritic and upper bathyal biofacies (Ingle, 1980).

Bathysiphon eocenicus Cushman and Hanna-Mallory, 1959, p. 105, pl. 1, fig. 4. Ecology: species of Bathysiphon have upper depth limits in the lower bathyal biofacies, $\geq 2000 \mathrm{~m}$ (Ingle, 1980).

Bathysiphon santecruis Cushman and Kleinpell, 1934, Cushman Lab. Foram. Res., Contr., v. 10, p. 1, pl. 1, figs. 1-2. Ecology: lower bathyal biofacies, $\geq 2000 \mathrm{~m}$ (Ingle, 1980).

Bifarina eleganata Plummer-Mallory, 1959, p. 204, pl. 29, fig. 3a,b. Range: from early Penutian to late Narizian Stages (Mallory, 1959).

Boldia hodgei Cushman and Schenck $=$ Cibicides hodgei Cushman and Schenck, 1928, Univ. Calif. Pub. Geol. Sci., v. 17, p. 315, pl. 45, figs. 3-5. Range: late Eocene (P15-P17) (Mallory, 1959; Donnelly, 1976; McDougall, 1980, 2008). Ecology: inner neritic biofacies, $\leq 50 \mathrm{~m}$ (Ingle, 1980). 
Bolivina advena Cushman, 1925, Cushman Lab. Foram. Res., Contr., v. 1, p. 29, pl. 5, figs. 1a-b. Range: Miocene, Saucesian Stage through Holocene (Kleinpell, 1938; Finger, 1990). Ecology: upper bathyal biofacies, 150-500 m (Ingle, 1980).

Bolivina advena ornata Cushman, 1925, Cushman Lab. Foram. Res., Contr., v. 1, p. 29, pl. 5, figs. 2 a-b. Range: Saucesian Stage to Holocene (Kleinpell, 1938; Finger, 1990). Ecology: upper bathyal biofacies, 150-500 m (Ingle, 1980).

Bolivina advena striatella Cushman, 1925, Cushman Lab. Foram. Res., Contr., v. 1, p. 30, pl. 5, fig. 3 Range: early and middle Miocene, Saucesian through Luisian Stages (Kleinpell, 1938). Ecology: upper bathyal biofacies, 150-500 m (Ingle, 1980).

Bolivina argentea Cushman, 1926, Contr. Cushman Lab. Foram. Res., v. 2, p. 42, pl. 6, fig. 5. Ecology: upper middle bathyal biofacies, 500-1500 m (Ingle, 1980); abundant in low oxygen waters $(\leq 1$ $\mathrm{ml} / \mathrm{l}$ ) and commonly found in the basins of the California borderland (Blake, 1981; Douglas, 1981).

Bolivina brevior Cushman, 1925, Cushman Lab. Foram. Res., Contr., v. 1, p. 31, pl. 5, figs. 8a-b. Range: Miocene, Saucesian Stage through Holocene (Kleinpell, 1938; Finger, 1990). Ecology: outer neritic biofacies, 50-150 m (Ingle, 1980).

Bolivina cuneiformis Kleinpell, 1938, p. 270, pl. 9, fig. 3. Range: middle Miocene, Relizian and Luisian Stages, questionably occurs in the late Miocene, Mohnian and Delmontian Stages (Kleinpell, 1938).

Bolivina decurtata Cushman, 1926, Cushman Lab. Foram. Res., Contr., v. 2, p. 44, pl. 6, figs. 7 a-b. Range: late Miocene, Mohnian Stage (Kleinpell, 1938; Pierce, unpublished, 1972).

Bolivina explicata lodoensis Mallory, 1959, p. 200, pl. 16, fig. 19a,b. Range: early and middle Eocene, Penutian through late Ulatisian Stages (P7-P10)(Mallory, 1959; McDougall, 2008).

Bolivina floridana Cushman, 1918, U.S. Geol. Survey Bull., no. 676, p. 49, pl. 10, fig. 4.

Bolivina guadeloupae Parker, 1964, Jour. Paleo., v. 38, p. 632, pl. 98, figs. 27-29.

Bolivina imbricata Cushman, 1925, Cushman Lab. Foram. Res., Contr., v. 1, p. 31. Range: Relizian Stage through Holocene (Kleinpell, 1938; Finger, 1990). Ecology: upper middle bathyal biofacies, 500-1500 m (Ingle, 1980).

Bolivina incrassata Reuss of Mallory, 1959, p. 201, pl. 16, fig. 24.

Bolivina jacksonensis Cushman and Applin, 1926, Amer. Assoc. Pet. Geol., Bull., v. 10, p. 167, pl. 7, figs. 3, 4.

Bolivina kleinpelli Beck, 1943, Jour. Paleo., v. 17, p. 606, pl. 107, fig. 39. Range: late Eocene, Narizian and Refugian Stages with rare occurrences in the middle Eocene, early Ulatisian Stage (Mallory, 1959; McDougall, 1980).

Bolivina marginata Cushman, 1918, U.S. Geol. Survey Bull., v. 676, p. 48, pl. 10, fig. 1. Range: late Eocene, Refugian through Holocene (Kleinpell, 1938; McDougall, 1980). Ecology: upper bathyal biofacies, 150-500 m (Ingle, 1980).

Bolivina pseudospissa Kleinpell, 1938, p. 279, pl. 21, fig. 6. Range: late Miocene, Mohnian through early Delmontian Stages (Kleinpell, 1938; Pierce, unpublished, 1972). Ecology: upper middle bathyal biofacies, 500-1500 m (Ingle, 1980).

Bolivina salinasensis Kleinpell, 1938, p. 280, pl. 9, fig. 6. Range: Miocene, late Saucesian through Mohnian Stages (Kleinpell, 1938).

Bolivina tumida Cushman, 1926, Cushman Lab. Foram. Res., Contr., v. 1, p. 32, pl. 5, figs. 9a-b. Range: Miocene, late Saucesian through Delmontian Stages (Kleinpell, 1938, 1980). 
Bolivina vaughani Natland, 1938, Bull., Scripps Inst. Ocean., Tech. Ser., v. 4, p. 146, pl. 5, fig. 11. Ecology: outer neritic biofacies, 50-150 m and less turbulent outer part of the inner neritic biofacies; common in the warmer tropical surface waters (Ingle, 1980; Ingle and Keller, 1980); abundant in the well oxygenated waters (5-6 ml/l) of the shelf (Blake, 1981; Douglas, 1981).

Bolivinoides delicatulus Cushman = Bolivinoides decorata (Jones) delicatulus Cushman , 1927, Cushman Lab. Foram. Res., Contr., v. 2, p. 90, pl. 12, fig. 8. Range: Late Cretaceous (Campanian) through late Paleocene (P6a)(McDougall, 2008; van Morkhoven and others, 1986). Ecology: primarily found at bathyal depths (van Morkhoven and others, 1986).

Buccella frigida (Cushman) = Pulvinulina frigida Cushman, 1920, U.S. Nat. Mus. Bull., v. 104, p. 12. Ecology: inner neritic biofacies, $\leq 50 \mathrm{~m}$ (Ingle, 1980).

Buccella mansfieldi oregonensis (Cushman, Stewart, and Stewart) = Eponides mansfieldi oregonensis Cushman, Stewart and Stewart, 1948, Oregon Dept. Geol. Min. Indust., Bull., no. 36, (1947), pt. 2, p. 48, pl. 6, figs. 4a-c. Range: Oligocene, Zemorrian Stage to Holocene (Finger, 1990; McDougall, 1980).

Bulimina alazaensis Cushman, 1927, Jour. Paleo., v. 1, p. 161, pl. 25, fig. 4. Range: earliest Eocene (P6b) into the Oligocene (P22) (McDougall, 2008; Tjalsma and Lohmann, 1983). Ecology: lower middle bathyal biofacies, 1,500-2,000 m (Ingle, 1980).

Bulimina bradburyi Martin, 1943, Stanford Univ. Pub., Geol. Sci., v. 3, p. 19, pl. 6, fig. 4a,b. Range: late Paleocene, Ynezian Stage into the early Oligocene (P4-P22)(Mallory, 1959; Tjalsma and Lohmann, 1983). Ecology: upper bathyal biofacies, 150-500 m (Ingle, 1980).

Bulimina cacumenata Cushman and Parker, 1936, Cushman Lab. Foram. Res., cont., v. 12, p. 40, pl. 7, fig. 3. Range: early Ynezian through late Bulitian Stages (Mallory, 1959); excellent marker for the Paleocene (Cushman, 1951).

Bulimina callahani Galloway and Morrey, 1931, Bull. Amer. Paleo., v. 15, p. 350, pl. 40, fig. 6. Range: Late Paleocene through middle Eocene, early Ulatisian Stage (P6a-P10) (Mallory, 1959; Tjalsma and Lohmann, 1983; van Morkhoven and others, 1986). Ecology: middle bathyal biofacies, 5001500 m (Ingle, 1980; Berggren and Aubert, 1983; van Morkhoven and others, 1986).

Bulimina excavata Cushman and Parker, 1936, Cushman Lab. Foram. Res., Contr., v. 12, p. 41, pl. 7 , fig. 4a-c. Range: restricted to the early Eocene, Penutian Stage (CP9-CP10, P7-P8) (Mallory, 1959; Almgren and others, 1988; McDougall, unpublished).

Bulimina inflata alligata Cushman and Laiming, 1931, Jour. Paleo., v. 5, p. 107, pl. 11, figs. 17a-b. Range: early Miocene, Saucesian Stage (Kleinpell, 1938, 1980).

Bulimina lirata Cushman and Parker, 1936, Cushman Lab. Foram. Res., Contr., v. 12, p. 43, pl. 8, fig.2a-c. Range: Penutian through Narizian stage (Mallory, 1959).

Bulimina macilenta Cushman and Parker, 1936, Cushman Lab. Foram. Res., Contr., v. 11, p. 47, pl. 7, figs. 7-8. Range: early Eocene, Penutian Stage through middle Eocene, early Narizian Stage (P7P15) (Mallory, 1959; McDougall, 2008; Tjalsma and Lohmann, 1983). Ecology: upper middle bathyal biofacies, 500-1500 m (Ingle, 1980).

Bulimina microcostata Cushman and Parker, 1936, Cushman Lab. Foram. Res., Contr., v. 12, p. 39, pl. 7, fig. 2a-c. Range: restricted to the Narizian Stage (P12-P15) (Mallory, 1959; McDougall, 2008). Ecology: upper middle bathyal biofacies, 500-1500 m (Ingle, 1980).

Bulimina midwayensis Cushman and Parker, Cushman Lab. Foram. Res., Contr., v. 12, p. 42, pl. 7, figs. 9-10. Range: Paleocene (P1 to P5)(Cushman, 1951; van Morkhoven and others, 1986). Ecology: middle neritic biofacies, 50-100 m (van Morkhoven and others, 1986).

Bulimina quadrata Plummer, 1926, Univ. Texas Bull., no. 2644, p. 72, pl. 4, figs. 4-5. 
Bulimina sculptilis Cushman, 1923, U.S. Geol. Survey, Prof. Paper 133, p.23, pl. 3, fig. 3. Range: late Eocene, Narizian through Refugian Stages (Mallory, 1959; McDougall, 2008).

Bulimina semicostata Nuttall, 1930, Jour. Paleo., v. 4, p. 274, pl. 23, figs. 15,16. Range: early Eocene through early Oligocene (P6b-P18) (Tjalsma and Lohmann, 1983; van Morkhoven and others, 1983). Ecology: upper middle bathyal biofacies, 500-1500 m (Ingle, 1980; van Morkhoven and others, 1986).

Bulimina trinitatensis Cushman and Jarvis, 1928, Cushman Lab. Foram. Res., Contr., v. 4, p. 102, pl. 14, fig. 12. Range: early Paleocene through late Eocene (P1-P16) (Tjalsma and Lohmann, 1983; van Morkhoven and others, 1986). Ecology: During the Eocene the upper depth limit may have been as shallow as 500-600 m (Barr and Berggren, 1980; Tjalsma and Lohmann, 1983; van Morkhoven and others, 1986).

Bulimina tuxapamensis Cole, 1928, Bull. Amer. Paleo., v. 14, p. 212, pl. 32, fig. 23. Range: late Paleocene through early middle Miocene with doubtful occurrences in middle Miocene (P6a-N9, ? N10-13)(Tjalsma and Lohmann, 1983; van Morkhoven and others, 1986). Ecology: upper bathyal biofacies, 150-500 m (Ingle, 1980; van Morkhoven and others, 1986).

Buliminella curta Cushman, 1925, Cushman Lab. Foram. Res., Contr., v. 1, p. 33, pl. 5, fig. 13. Range: Oligocene, Zemorrian Stage, through Holocene (Finger, 1990). Ecology: outer neritic biofacies, 50-150 m (Ingle, 1980); low oxygen conditions (Blake, 1991).

Buliminella elegantissima d'Orbigny-Finger, 1990, p. 70-71, plate-figs. 1-8. Range: Oligocene, Zemorrian Stage through Holocene (Finger, 1990). Ecology: inner neritic biofacies, $\leq 50 \mathrm{~m}$ (Ingle, 1980; Smith, 1964).

Buliminella grata Parker and Bermudez, 1937, Jour. Paleo., v. 11, p. 515, pl. 59, fig. 6a-c. Range: early Paleocene through middle Miocene (P1-N12)(van Morkhoven and others, 1986). Ecology: bathyal species; maximum abundances occurred at depths of 1-2 km in Atlantic (Tjalsma and Lohmann, 1983; van Morkhoven and others, 1986).

Buliminella robertsi Howe and Ellis, 1939, Louisiana Dept. Conservation, Geol. Survey, Geol. Bull., no. 14, p. 63, pl. 8, figs. 32-33. Range: latest Paleocene through late Eocene (P6a-P15) (Tjalsma and Lohmann, 1983). Ecology: outer neritic biofacies, 50-150 m (Ingle, 1980).

Buliminella subfusiformis Cushman, 1925, Cushman Lab. Foram. Res., contr., v. 1, p. 33, pl. 5, fig. 12. Range: Oligocene, Zemorrian Stage through Holocene (Finger, 1990; Kleinpell, 1938). Ecology: upper bathyal biofacies, 150-500 m, associated with low oxygen conditions (Ingle, 1980).

Cancris malloryi Smith, 1957, p. 185, pl. 28, fiigs. 3a-c, 5a-c.

Cassidulina californica Cushman and Hughes, 1925, Cushman Lab. Foram. Res., Contr., v. 1, p. 12, pl. 2, fig. 1. Range: Miocene to Holocene (Finger, 1990). Ecology: upper bathyal biofacies but may be transitional between outer neritic and upper bathyal biofacies (Ingle, 1980).

Cassidulina crassipunctata Cushman and Hobson, 1925, Cushman Lab. Foram. Res., Contr., v. 11, p. 63, pl. 9, fig. 10. Range: late Eocene, Refugian Stage through Oligocene, Zemorrian Stage (P16P22)(Kleinpell, 1938).

Cassidulina panzana Kleinpell, 1938, p. 335, pl. 8, fig. 9. Range: early and middle Miocene, late Saucesian through late Luisian stages (Kleinpell, 1938).

Cassidulina pulchella d'Orbigny-Kleinpell, 1938, p. 335-336, pl. 10, fig. 9. Range: middle Miocene, Luisian through early Mohnian Stages (Finger, 1990; Kleinpell, 1938, 1980). Ecology: upper bathyal biofacies, 150-500 m(Ingle, 1980).

Cassidulina transluscens Cushman and Hughes, 1925, Cushman Lab. Foram. Res., Contr., v. 1, p. 15, pl. 2, fig. 5. Ecology: upper bathyal biofacies, but may be transitional between the outer shelf and upper bathyal biofacies (Ingle, 1980). 
Cassidulinoides cornuta $($ Cushman $)=$ Virgulina cornuta Cushman, 1913, U.S. Nat. Mus., Proceedings, v. 44, no. 1973, p. 637 pl. 1, fig. 1. Range: Pliocene to Holocene (Haller, 1980; White, 1956).

Caucasina schencki $($ Beck $)=$ Bulimina schencki Beck, 1943, Jour. Paleo., v. 17, p. 605, pl. 107, figs.

28, 33. Range: late Eocene, late Narizian through Refugian Stages (P15-P17) (McDougall, 1980, 2008). Ecology: outer neritic biofacies, 50-150 m (McDougall, 1980).

Ceratobulimina perplexa (Plummer)-Mallory, 1959, p. 228, pl. 19, fig. 14a-b; pl. 40, fig. 8a-c.

Chilostomella cylindroides Reuss-Mallory, 1959, p. 243, pl. 22, fig. 3. Ecology: upper bathyal biofacies, $150-500 \mathrm{~m}$ (Ingle, 1980).

Chilostomella czizeki Reuss, 1850, Kaiserliche Akad. Wiss. Wien, Math.-Nat. Classe, Denkschrift, v. 1, p. 380 , pl. 48 , figs. $13 \mathrm{a}-\mathrm{d}$.

Chilostomella oolina Schwager, 1878, Uff. Geol. (Reale Com. Geol. Italiana), Boll. Roma, Italia, v. 9, p. 527, pl. 1, fig. 16. Ecology: upper bathyal biofacies, 150-500 m (Ingle, 1980).

Chilostomella ovoidea Reuss-Kleinpell, 1938, p. 33, pl. 22, fig. 8; pl. 31, fig. 4. Ecology: upper middle bathyal biofacies in anaerobic bottom conditions (Ingle, 1980).

Chrysalongonium elongatum Cushman and Jarvis, 1934, Cushman Lab. Foram. Res., Contr., v. 10, p. 73, pl. 10, figs. 10-11.

Chrysalongonium laeve Cushman and Bermudez, 1936, Cushman Lab. Foram. Res., Contr., v. 12, p. 27, pl. 5, figs. 1, 2.

Chrysalongonium longiscatatum Cushman and Jarvis, 1934, Cushman Lab. Foram. Res., Contr., v. 10, p. 74, pl. 10, fig. 12 .

Chrysalongonium tenuicostatum Cushman and Bermudez, 1936, Cushman Lab. Foram. Res., Contr., v. 12, p. 27, pl. 5, figs. 3-5.

Cibicides americanus Cushman crassiseptus Cushman and Laiming, Jour. Paleo., v. 5, p. 119, pl. 14, fig. 7. Range: Oligocene through early Miocene, Zemorrian through early Saucesian Stages (P18-N4) (Kleinpell, 1938).

Cibicides beatus Martin, 1943, Stanford Univ. Pub. Geol. Sci., . 3, p. 31, pl. 8, figs. 6a-c.

Cibicides felix Martin, 1943, Stanford Univ. Pub. Geol. Sci., . 3, p. 31, pl. 8, figs. 7a-c. Range: late Ynezian through early Penutian Stages (Mallory, 1959).

Cibicides fletcheri Galloway and Wissler, 1927, Jour. Paleo., v. 1, p. 64, pl. 10, figs. 8-9. Ecology: UDL is in the inner neritic biofacies along the Eastern Pacific Margin (Ingle, 1980; Lankford and Phleger, 1973).

Cibicides floridanus (Cushman)-Kleinpell, 1938, p. 353-354.

Cibicides kernensis Cook in Mallory, 1959, p. 266, pl. 24, fig.2a-c.

Cibicides kleinpelli Smith, 1957, p. 193, pl. 29, fig. 9a-c.

Cibicides madrugaensis Cushman and Bermudez, 1948, Cushman Lab. Foram. Res., Contr., v. 24, p. 87, pl. 15, figs. 10-12.

Cibicides mckannai Galloway and Wissler, 1927, Jour. Paleo., v. 1, p. 65, pl. 10, figs. 5-6. Range: Middle Miocene to Holocene (Finger, 1990). Ecology: upper bathyal biofacies, 150-500 m (Ingle, 1980).

Cibicides ouachitaensis alhambrensis Smith, 1957, p. 194, pl. 32, figs. 1a-c, 2a-c, 5a-c.

Cibicides pachecoensis Smith, 1957, p. 194, pl. 3, figs. 8a-c, 11a-c. Range: Paleocene, Ynezian and Bulitian Stages however the Bulitian occurrence is questioned (Mallory, 1959; McDougall, unpublished).

Cibicides pseudoungerianus $($ Cushman $)=$ Truncatulina pseudoungeriana Cushman, 1922, U.S. Geol. Survey, Prof. Paper 129, p. 97, pl. 20, fig. 9. Range: middle Eocene to Oligocene (Douglas, 1973). 
Cibicidoides alleni (Plummer) = Truncatulina alleni Plummer, 1926, Texas Univ. Bull., no. 2644, p. 144, pl. 10, fig. 4. Range: Paleocene through early Eocene (P1 to P6b) (van Morkhoven and others, 1986). Ecology: middle and outer neritic biofacies, 50-150 m(van Morkhoven and others, 1986).

Cibicidoides cocoaensis $($ Cushman $)=$ Eponides cocoaensis Cushman, 1928, Cushman Lab. Foram. Res., Contr., v. 4, p. 73, pl. 10, fig. 2.

Cibicidoides coalingensis (Cushman and Hanna) = Anomalina coalingensis Cushman and Hanna, 1927, Calif. Acad. Sci. Proc., $4^{\text {th }}$ ser., v. 16, p. 221, pl. 14, figs. 10-12. Range: Eocene (P6b-P17) (McDougall, 2008). Ecology: outer neritic biofacies, 50-150 m (Ingle, 1980).

Cibicidoides dayi $($ White $)=$ Planulina dayi White, 1928, Jour. Paleo., v. 2, p. 300, pl. 4, figs. 3a-c. Range: Late Cretaceous (Campanian) through late Paleocene (P5) (van Morkhoven and others, 1986). Ecology: primarily a bathyal and abyssal species (van Morkhoven and others, 1986).

Cibicidoides eocaenus (Gumbel)—van Morkhoven and others, 1986, p. 256-263, pl. 86A, figs. 1-4; pl. 86B, figs. 1-2; pl. 86C figs. 1-3; pl. 86D, figs. 1-2. Range: early Eocene (P6b) through late Oligocene (P22) (van Morkhoven and others, 1986). Ecology: outer neritic to abyssal depths depending on form present (Ingle, 1980; van Morkhoven and others, 1986).

Cibicidoides eponidiformis (Martin) = Cibicides eponidiformis Martin, 1943, Stanford Univ. Pub. Geol. Sci., v. 3, p. 50, pl. 6, fig. 7a, b, c. Range: Paleocene, late Ynezian Stage through at least late Narizian Stage (Mallory, 1959).

Cibicidoides fortunatus (Martin) = Cibicides fortunatus Martin, 1943, Stanford Univ. Pub. Geol. Sci., v. 3, p. 31, pl. 8, fig. 5a-c. Range: early Eocene, Penutian Stage (P6b-P9) (McDougall, 2008). Ecology: upper bathyal biofacies, 150-500 m (Ingle, 1980).

Cibicidoides grimsdalei $($ Nuttall $)=$ Cibicides grimsdalei Nuttall, 1930, Jour. Paleo., v. 4, p. 291, pl. 25, figs. 7,8,11. Range: early Eocene through Miocene (P8-N4) (van Morkhoven and others, 1986). Ecology: lower bathyal biofacies, 1,500-2,000 m (Ingle, 1980; Tjalsma and Lohmann, 1983).

Cibicidoides hyphalus $($ Fisher) = Anomalinoides hyphalus Fisher, 1969, Paleo., v. 12, p. 197-198, tf.

31a-c. Range: Late Cretaceous (late Maastrichtian) through late Paleocene (P5) (van Morkhoven and others, 1986). Ecology: outer neritic through bathyal biofacies (van Morkhoven and others, 1986).

Cibicidoides laurisae (Mallory) $=$ Cibicides laurisae Mallory, 1959, p. 267, pl. 24, fig. 81a-c. Range: middle Eocene (P10) through late Oligocene (P22) (van Morkhoven and others, 1986).

Cibicidoides martinezensis Cushman and Barksdale, 1930, Stanford Univ. Pub. Geol. Sci., v. 1, p. 88, pl. 12, fig. 9a-c. Range: early Eocene, Bulitian Stage through middle Eocene, early Narizian Stage (CP9-CP14, P6b-P14) (Almgren and others, 1988; Mallory, 1959).

Cibicidoides praecursorius $($ Schwager $)=$ Cibicides praecursoria $($ Schwager $)$-Mallory, 1959, p. 269, pl. 32, fig. 10. Range: Paleocene, restricted to the Ynezian Stage (Mallory, 1959).

Cibicidoides praemundulus Berggren and Edwards in van Morkhoven and others, 1986, p. 264-266, pl. 87, figs. 1-2. Range: early Eocene through late Oligocene (P6b-P22). Ecology: primarily a lower bathyal and abyssal form, but occurs sporadically in middle bathyal sediments (van Morkhoven and others, 1986).

Cibicidoides subspiratus $($ Nuttall) = Cibicides subspiralis Nuttall, 1930, Jour. Paleo., v. 4, p. 292, pl. 25, figs. 9, 10. 14. Range: late early Eocene through late middle Eocene (P9-P13) (van Morkhoven and others, 1986). Cibicides fortunatus, which maybe a synonym occurs in the early Eocene (P6b-P8)(McDougall, unpublished). Ecology: bathyal and abyssal species (van Morkhoven and others, 1986). 
Cibicidoides velascoensis $($ Cushman $)=$ Anomalina velascoensis Cushman, 1925, Cushman Lab. Foram. Res., Contr., v. 1, p. 21, pl. 3, figs. 3a-c. Range: Late Cretaceous (Campanian) through Paleocene (P5) (van Morkhoven and others, 1986). Ecology: upper bathyal biofacies, 150-500 $\mathrm{m}$ (van Morkhoven and others, 1986).

Cibicidoides venzuelanus $($ Nuttall) = Cibicides venezuelanus Nuttall, 1935, Jour. Paleo., v. 9, p. 131, pl. 15, figs. 25-27.

Citharina plummoides $($ Plummer $)=$ Vaginulina plummoides Plummer, 1926, Texas Univ. Bull., no. 2644, p. 113, pl. 6, fig. 6.

Clavulina anglica $($ Cushman $)=$ Pseudoclavulina anglica Cushman 1936, Cushman Lab. Foram. Res., Spec. Pub., no. 6, p. 18, pl. 3, fig. 5. Range: early Paleocene through early Eocene (P1-early P9) (King, 1989; McDougall, 2008). Ecology: normal marine shelf (Murray, 1991).

Clavulinoides californicus (Mallory) = Clavulinoides californicus Mallory, 1959, p. 123, pl. 4, fig. 6a-b. Range: Paleocene through middle Eocene, early Ynezian through early Narizian Stages (Mallory, 1959). Ecology: outer neritic biofacies, 50-150 m (Ingle, 1980).

Clavulinoides midwayensis Cushman, 1936, Cushman Lab. Foram. Res., Spec. Pub., no. 6, p. 21, pl. 3, figs. 9, 15. Ecology: outer neritic biofacies, 50-150 m (Ingle, 1980; van Morkhoven and others, 1986).

Coleites recticulosus $($ Plummer $)=$ Pulvinulina reticulosa Plummer, 1926, Texas Univ. Bull., no. 2644, p. 152 , pl. 12, fig. 5 .

Concavella gyroidinaformis (Cushman and Goudkoff) $=$ Pulvinulinella gyroidinaformis Cushman and Goudkoff, 1938, Cushman Lab. Foram. Res., Contr., v. 14, p. 1, pl. 1, figs. 1-2. Range: middle and late Miocene, late Luisian through Mohnian Stages (Kleinpell, 1938; Pierce, unpublished, 1972; Finger, 1990). Ecology: upper middle bathyal biofacies, 500-1500 m; frequently associated with low oxygen conditions (Ingle, 1980; Blake, 1991).

Coryphostoma midwayensis $($ Cushman $)=$ Bolivina midwayensis Cushman, 1935, Cushman Lab. Foram. Res., Spec. Pub., v. 6, p. 50, pl. 7, fig. 12. Range: Late Cretaceous through Paleocene (P5) (van Morkhoven and others, 1986). Ecology: outer neritic biofacies, 50-150 m (van Morkhoven and others, 1986).

Cribrostomoides cretacea Cushman and Goudkoff, 1944, Cushman Lab. Foram. Res., Contr., v. 20, p. 54, pl. 9, fig. 4.

Cribrostomoides trinitatensis Cushman and Jarvis, 1928, Cushman Lab. Foram. Res., Contr., v. 4, p. 91, pl. 12, fig. 12a-b. Range: Late Cretaceous (Maastrichtian) through the late Paleocene, planktic foraminiferal zone P6a (Kaminski and others, 1988).

Cyclammina clarki (Hanna)= Nonionina clarki Hanna, 1923, Univ. Calif. Pub. Geol. Sci., v. 14, p. 324, pl. 59, fig. 2. Range: Oligocene, Zemorrian Stage through early Miocene, early Saucesian Stage (Kleinpell, 1938). Ecology: lower middle bathyal biofacies, 1,500-2,000 m (Ingle, 1980).

Cyclammina pacifica Beck, 1943, Jour. Paleo., v. 17, p. 591, pl. 98, figs. 2,3. Range: late Ulatisian through late Narizian stages (Mallory, 1959). Ecology: lower middle bathyal biofacies, 1,5002,000 m (Ingle, 1980).

Cyclammina samanica Berry, 1928, Eclog. Geol. Helv., v. 21, p. 393, tfs. 5a-c. Range: late Bulitian through late Narizian Stages (Mallory, 1959). Ecology: lower middle bathyal biofacies, 1,5002,000 m (Ingle, 1980).

Cyclammina simiensis Cushman and McMasters, 1936, Jour. Paleo., v. 10, p. 509, pl. 74, fig. 3a,b. Darbayella wilcoxensis Cushman and Garrett, 1939, Cushman Lab. Foram. Res., Contr., v. 15, p. 79, pl. 13, figs. 11-12. 
Dentalina basiplanata Cushman, 1938, Cushman Lab. Foram. Res., Contr. , v. 14, p. 38, pl. 6, figs. 1112.

Dentalina colei Cushman and Dusenbury, 1934, Cushman Lab. Foram, Res., Contr., v. 10, p. 54, pl. 7 , figs. 10-12.

Dentalina communis (d'Orbigny) -Mallory, 1959, p. 162, pl. 12, fig. 11; pl. 41, fig. 6.

Dentalina consobrina (d'Orbigny)—Mallory, 1959, p. 163, pl. 12, fig. 12; pl. 41, fig. 5.

Dentalina delicatula Cushman, 1938, Cushman Lab. Foram. Res., Contr., v. 14, p. 40, pl. 6, figs. 19-20.

Dentalina globulicauda Gumbel, 1868, K. Bayer. Akad. Wiss. Munchen, Math.-Physik. Cl., Abh., Munchen, Deutschland, Bd. 10, Abt. 2, p. 623, pl. 1, fig. 38.

Dentalina hexacostata Howe, 1939, Louisiana Geol. Survey Bull., v. 14, p. 44, pl. 5, fig. 13. Range: late Ulatisian through early Narizian Stages (Mallory, 1959).

Dentalina insulsa Cushman, 1947, Cushman Lab. Foram. Res., Contr., v. 23, p. 84, pl. 18, figs. 6-7.

Dentalina intorta d'Orbigny, 1846, Foraminiferes fossiles du bassin tertiaire de Vienne Gide et. Comp., p. 44 , pl. 1, figs. 50-51.

Dentalina jacksonensis (Cushman and Applin) = Nodosaria jacksonensis Cushman and Applin, 1926,

Amer. Assoc. Petr. Geol. Bull., v. 10, p. 170, pl. 7, figs. 14-16.

Dentalina pseudobliquestriata (Plummer) = Nodosaria pseudo-obliquestriata Plummer, 1926, Texas

Univ. Bull., no. 2644, p. 87, pl. 4, fig. 11.

Dentalina soluta Reuss, 1851, Deutsch. Geol. Ges., Zeitschr., Deutschland, Bd. 3, p. 60, pl. 3, fig. 4.

Dentalina spinosa d'Orbigny-Mallory, 1959, p. 167, pl. 12, fig. 26.

Discorbis baintoni Mallory, 1959, p. 228, pl. 19, fig. 16a-c. Range: Penutian through Ulatisian Stages (planktic foraminiferal zones P7 through P10, possibly P11) (Mallory, 1959). Ecology: inner neritic biofacies, $\leq 50 \mathrm{~m}$ (Ingle, 1980).

Dorothia bulletta (Carsey) = Gaudryina bulletta Carsey, 1926, Texas Univ. Bull, no. 2612, p. 28, pl. 4, fig. 4. Range: Cretaceous to early Penutian Stage with rare occurrences noted in the early Ulatisian and the Narizian Stages (Mallory, 1959; Sliter, 1968).

Dorothia principiensis Cushman and Bermudez, 1936, Cushman Lab. Foram. Res., Contr., v. 12, p. 57, pl. 10, figs. 3-4. Range: late Ynezian through late Narizian Stages (Mallory, 1959).

Dorothia retusa $($ Cushman $)=$ Gaudryina retusa Cushman, 1926, Amer. Assoc. Pet. Geol. Bull., v. 10, p. 588, pl. 16, fig 10a-b.

Eggerella elongata Blaisdell, 1965, Cushman Found. Foram. Res., Contr., v. 16, p. 27, pl. 2, figs. 1-3. Range: late Ulatisian through Refugian Stages (?P10, P12-P16) (Mallory, 1959; McDougall, 2008).

Eggerella subconica Parr, 1950, B.A.N.Z. Antarct. Res. Exped. 1929-31, Rept., Adelaide, v. 5, p. 281, pl. 5, fig. 22. Range: early Narizian through late Narizian Stages (Mallory, 1959). Ecology: upper middle bathyal biofacies, 500-1500 m (Ingle, 1980).

Ellipsoglandulina labiata (Schwager) = Glandulina labiata Schwager, 1866, Norara-Exped., Geol. Thril, v. 2, p. 237, pl. 6, fig. 77.

Ellipsoglandulina multicostata = Daucina multicostata Galloway and Morrey, 1929, Bull. Amer. Paleo., v. 15, p. 42, pl. 6, fig. 13.

Ellipsonodosaria alexanderi Cushman, 1936, Cushman Lab. Foram. Res., Contr., v. 12, p. 52, pl. 9, figs. 6-9.

Elphidium californicum Cook in Mallory, 1959, p. 184, pl. 15, fig. 10a,b; pl. 33, fig. 8a,b. Range: late Bulitian through late Ulatisian Stages (Mallory, 1959). Ecology: inner neritic biofacies, $\leq 50 \mathrm{~m}$ (Ingle, 1980). 
Epistominella bradyana $($ Cushman $)=$ Pulvinulinella bradyana Cushman, 1927, Bull, Scripps Inst. Ocean., Tech. Ser., v. 1, p. 165, pl. 5, figs. 11-13. Ecology: outer neritic biofacies, 50-150 m (Smith, 1964).

Epistominella capitanensis Cushman and Kleinpell = Pulvinulinella capitanensis Cushman and Kleinpell, 1938, Cushman Lab. Foram. Res., Contr., v. 10, p. 16, pl. 3, figs. 3a-c. Range: middle and late Miocene, late Luisian through early Mohnian Stages, and questionably into the Delmontian Stage (Kleinpell, 1938; Finger, 1990). Ecology: transitional between the upper bathyal and upper middle bathyal biofacies, 150-500 and 500-1500 m(Ingle, 1980).

Epistominella pacifica $($ Cushman $)=$ Pulvinulinella pacifica Cushman, 1927, Bull, Scripps Inst. Ocean., Tech. Ser., v. 1, p. 165, pl. 5, figs. 14, 15. Range: middle Miocene, Relizian Stage to Holocene (Kleinpell, 1938; Finger, 1990). Ecology: upper middle bathyal biofacies, 500-1500 m (Ingle, 1980).

Epistominella smithi $($ Stewart and Stewart $)=$ Pulvinulinella smithi Stewart and Stewart, 1930, Jour. Paleo., v. 4, p. 70, pl. 9, fig. 4. Range: Miocene to Pliocene (Finger, 1990). Ecology: transitional between upper bathyal and upper middle bathyal biofacies, 150-500 and 500-1500 m (Ingle, 1980); low oxygen conditions (Blake, 1991).

Epistominella subperuviana $($ Cushman $)=$ Epistominella relizensis Kleinpell, 1938, p. 329-330, pl. 10, figs. 10a-c. Range: Oligocene, late Saucesian Stage through Holocene (Kleinpell, 1938; Bandy and Arnal, 1969; Finger, 1990). Ecology: transitional between the outer neritic and upper bathyal biofacies (Ingle, 1980); associated with temperatures of $7-13{ }^{\circ} \mathrm{C}$, a salinity of $34.6 \%$ and an oxygen content of less than $1 \mathrm{ml} / \mathrm{l}$ (Resig, 1981).

Eponides lodoensis Martin, 1943, Stanford Univ. Pub. Geol. Sci., v. 3, p. 22, pl. 6, fig. 8a-c. Range: Bulitian through late Penutian Stages (Mallory, 1959).

Eponides lotus $($ Schwager $)=$ Pulvinulinella lotus Schwager, 1883, Paleontographica, v. 30, Pal. Theil., p. 132 , pt. 28 , fig. 9 .

Eponides mexicanus $($ Cushman $)=$ Pulvinulina mexicana Cushman, 1925, Amer. Assoc. Petr. Geol., Bull., v. 9, p. 300, pl. 7, figs. 7-8. Range: middle Eocene through early Oligocene (P7-P22) (Kleinpell, 1938; Mallory, 1959; McDougall, 2008) . Ecology: inner neritic biofacies, $\leq 50 \mathrm{~m}$ (Ingle, 1980).

Eponides plummerae Cushman, 1948, Cushman Lab. Foram. Res., Contr., v. 24, p. 44, pl. 8, fig. 9. Range: middle and late Paleocene (P3-P5)(Berggren and Aubert, 1976; McDougall, 2008). Eponides waltonensis Applin and Jordan, 1945, Jour. Paleo., v. 19, p. 142, pl. 19, fig. 5.

Fissurina alveolata (Brady) = Lagena alveolata Brady, 1884, Report on the Scientific Results of the Voyage of the H.M.S. Challenger during the years 1873-1876, Zoology, v. 9, p. 487, pl. 60, figs. $30,32$.

Fissurina marginata $($ Montagu $)=$ Vermiculum marginatum Montagu, 1803, Testacea Britannica or natural history of British Shells, marine, land and fresh-water, including the most minute, J.S. Hollis, Romsey, England, p. 524.

Fissurina orbignyana Sequenza, 1862, Deii terreni Terziarii del distretto di Messina; Parte II Descriptione dei foraminiferi monotalamici delle marine mioceniche del distretto de Messina $\mathrm{T}$. Capra, p. 66, pl. 2, figs. 25-26.

Frondicularia frankei Cushman, 1936, Cushman Lab. Foram Res., Contr. v. 12, p. 18, pl. 4, figs. 6-7. Range: late Paleocene, Ynezian Stage (P4)(Laiming, 1939; McDougall, 2008).

Fursenkoina bramletti (Galloway and Morrey) = Virgulina bramletti Galloway and Morrey, 1929, Bull. Amer. Paleo., v. 15, p. 37, pl. 5, fig. 14a,b. Range: late middle Eocene, early Narizian Stage through early Miocene, early Saucesian Stage (P12-N4) (Kleinpell, 1938; Mallory, 1959). 
Fursenkoina californiensis $($ Cushman $)=$ Virgulina californiensis Cushman, 1925, Cushman Lab. Foram. Res., Contr., v. 1, p. 32, pl. 5, fig. 11. Range: Miocene, Saucesian through early Delmontian Stages (Kleinpell, 1938). Ecology: upper bathyal biofacies, 150-500 m; low oxygen indicator (Ingle, 1980).

Fursenkoina californiensis grandis (Cushman and Kleinpell) = Virgulina californiensis Cushman grandis Cushman and Kleinpell, 1934, Cushman Lab. Foram. Res., Contr. , v. 10, p. 9, pl. 1, figs. 15-16. Range: middle and late Miocene, Mohnian through Delmontian stages with rare occurrences in the Luisian Stage (Kleinpell, 1938).

Fursenkoina californiensis ticensis (Cushman and Kleinpell) = Virgulina californiensis Cushman ticensis Cushman and Kleinpell, 1934, Cushman Lab. Foram. Res., Contr. , v. 10, p. 10, pl. 1, fig. 17. Range: middle Miocene, early Mohnian Stage (Kleinpell, 1938).

Gaudryina arenaria Galloway and Wissler, 1927, Jour. Paleo., v. 1, p. 68, pl. 11, fig. 5. Ecology: outer neritic biofacies, 50-150 m (Bandy, 1961; Ingle, 1980).

Gaudryina coalingensis $($ Cushman and Hanna) = Gaudryina jacksonensis coalingensis Cushman and Hanna, 1927, California Acad. Sci., Proc., ser. 4, v. 16, p. 212, pl. 13, fig. 7.

Gaudryina laevigata Franke, 1914, Geol. Ges., Zeitschr., A., Abh., Deutschland, Bd. 66, Heft 3, p. 431, pl. 27, figs. 1-2. Ecology: outer neritic biofacies (Ingle, 1980).

Gaudryina pachecoensis Smith (1957). Ecology: outer neritic biofacies, 50-150 m (Ingle, 1980).

Gaudryina pyramidata $($ Cushman $)=$ Gaudryina laevigata pyramidata Cushman, 1926, Amer. Assoc. Pet. Geol. Bull., v. 10, p. 587, pl. 16, fig. 8.

Glandulina laevigata (d'Orbigny) = Nodosaria (Glanduline) laevigata d'Orbigny, 1826, Tableau methodique de la classe des Cephalododes, Ann. Sci. Nat., Paris, France, ser. 1, tome 7, p. 252, pl. 10, figs. 1-3. Ecology: upper middle bathyal biofacies, 500-1500 m (Ingle, 1980).

Globobulimina galliheri $($ Kleinpell $)=$ Bulimina galliheri Kleinpell,1938, p. 253, pl. 17, figs. 2, 5 . Range: middle Miocene, late Luisian through early Mohnian Stages (Kleinpell, 1938). Ecology: upper middle bathyal biofacies, 500-1500 m (Ingle, 1980).

Globobulimina montereyana $($ Kleinpell $)=$ Bulimina montereyana Kleinpell, 1938 p. 254-255, pl. 12, fig. 13. Range: middle and late Miocene, Luisian through early Delmontian Stages (Kleinpell, 1938).

Globobulimina pacifica Cushman, 1927, Cushman Lab. Foram. Res., Contr., v. 3, p. 67, pl. 14, fig. 12. Range: middle and late Eocene, late Ulatisian through late Narizian Stages with rare occurrences in the late Bulitian Stage (Mallory, 1959). Ecology: transitional between outer neritic and upper bathyal biofacies associated with the shallow oxygen minimum zone in the upper bathyal biofacies (Ingle, 1980; Ingle and Keller, 1980).

Globobulimina pseudotorta $($ Cushman $)=$ Bulimina pseudotorta Cushman, 1926, Cushman Lab. Foram . Res., Contr., v. 2, p. 55, pl. 7, fig. 3. Range: middle Miocene, Relizian and Luisian Stages through Holocene (Kleinpell, 1938; Finger, 1990). Ecology: upper middle bathyal biofacies, 500-1500 m (Ingle, 1980).

Globocassidulina globosa $($ Hantken $)=$ Cassidulina globosa Hantken-Mallory, 1959, p. 226, pl. 33,

fig. 11a,b. Range: questionably first appears in the late Paleocene, (P4) and ranges through late Eocene, late Refugian Stage (Mallory, 1959; Tjalsma and Lohmann, 1983; McDougall, 1980, 2008). Ecology: outer neritic biofacies, 50-150 m (Ingle, 1980).

Globulina gibba d'Orbigny, 1826, Ann. Sci. Nat., v. 7, p. 266, no. 10, modeles no. 63.

Glomospira charoides-Kaminiski and others, 1988, p. 185, pl. 3, figs. 14-15. Range: Paleocene through early Eocene with questionable occurrences through the late Eocene (P4-P7,?P8- 
P15)(Mallory, 1959; Kaminski and others, 1988; McDougall, 2008). Ecology: lower bathyal biofacies, $\geq 2000 \mathrm{~m}$ (Ingle, 1980).

Gonatosphaera eocenica Mallory, 1959, p. 225, pl. 18, fig. 19. Range: early and middle Eocene, Penutian through early Ulatisian Stages (P7-P10)(Mallory, 1959; McDougall, 2008).

Guttulina irregularis d'Orbigny-Mallory, 1959, p. 177, pl. 14, fig. 13. Ecology: outer shelf biofacies, 50-150 m (Ingle, 1980).

Guttulina problema d'Orbigny, 1826, Ann. Sci. Nat., v. 7, p. 266. Ecology: outer shelf biofacies, 50-150 m (Ingle, 1980).

Gyroidina condoni $($ Cushman and Schenck $)=$ Eponides condoni Cushman and Schenck, 1928, Calif. Univ., Dept. Geol. Sci., Bull., v. 17, p. 313, pl. 44, figs. 6-7.

Gyroidina globosus $($ Hagenow $)=$ Gyroidinoides globosus $($ Hagenow $)$-van Morkhoven and others, 1986, p. 329-330, pl. 107, figs. 1-3. Range: Late Cretaceous (Campanian) through early Eocene (P6b) (van Morkhoven and others, 1986). Ecology: bathyal and abyssal (van Morkhoven and others, 1986).

Gyroidina obliquata Cushman and McMasters = Gyroidina orbicularis d'Orbigny obliquata Cushman and McMasters 1936, Jour. Paleo., v. 10, p. 514, pl. 76, fig. 4a-c.

Gyroidina octocamerata Cushman and Hanna = Gyroidina soldanii d'Orbigny octocamerata Cushman and Hanna-Mallory (1959). Ecology: lower bathyal biofacies, $\underline{1,500}-\underline{2,000} \mathrm{~m}$ (Ingle, 1980).

Gyroidina orbicularis d'Orbigny, 1826, Ann. Sci. Nat., v. 7, p. 278, modeles no. 13. Ecology: lower bathyal biofacies, $\underline{1,500}-\underline{2,000} \mathrm{~m}$ (Ingle, 1980).

Gyroidina planulata Cushman and Renz, 1941, Cushman Lab. Foram Res., Contr., v. 17, p. 23, pl. 4, fig. 1. Ecology: upper bathyal biofacies, 150-500 m (Ingle, 1980).

Gyroidina relizana Kleinpell, 1938, p. 315, pl. 10, fig. 11. Range: middle Miocene, late Relizian Stage (Kleinpell, 1938)

Gyroidina soldanii d'Orbigny, 1826, Ann. Sci. Nat., ser. 1, v. 7, p. 278. Ecology: lower bathyal biofacies, $\geq 2000 \mathrm{~m}$ (Ingle, 1980; Ingle and Keller, 1980).

Hanzawaia ammophila (Gumbel)—van Morkhoven and others, 1986, p. 168-171, pl. 56, figs. 1-3. Range: latest Paleocene, late Bulitian Stage through middle Miocene (P6a-N11) (Mallory, 1959; van Morkhoven and others, 1986). Ecology: outer neritic biofacies, 50-150 m (Ingle, 1980; van Morkhoven and others, 1986).

Hanzawaia blanpiedi $($ Toulmin $)=$ Cibicides blanpiedi Toulmin, 1941, Jour. Paleo., v. 15, p. 609, pl. 83, figs. 11-13. Ecology: inner neritic biofacies, $\leq 50 \mathrm{~m}$ (Ingle, 1980).

Hanzawaia illingi (Nuttall) = Truncatulina illingi Nuttall, 1928, Geol. Soc. London, Quart. Jour., v. 84, p. 99, pl. 7, figs. 11, 17; p. 99, tf. 5. Ecology: outer neritic biofacies, 50-150 m (Ingle, 1980).

Hanzawaia mauricensis (Howe and Roberts) = Cibicides mauricensis Howe and Roberts, 1939, Louisiana Dept. of Conservation, Geol. Survey Geol. Bull., no. 14, p. 87, pl. 13, figs. 4-5. Range: early Eocene, Penutian Stage, through middle Eocene, Ulatisian Stage (P7-P10) (Bandy, 1949; Mallory, 1959; McDougall, 2008).

Haplophragmoides eggeri Cushman, 1926, Amer. Assoc. Petr. Geol., Bull., v. 10, pl. 15, fig. 1. Range: Paleocene through middle Eocene, Ynezian through early Narizian Stages (Mallory, 1959).

Haplophragmoides excavatus Cushman and Waters, 1927, Cushman Lab. Foram. Res., Contr., v. 2, p. 82 , pl. 10, fig. 3a,b.

Haplophragmoides glabrus Cushman and Waters, 1927, Cushman Lab. Foram. Res., Contr., v. 2, p. 83, pl. 10, fig. 6a,b.

Haplophragmoides obliquicameratus Marks, 1951, Cushman Lab. Foram. Res., Contr., v. 2, p. 35, pl. 5, fig. 1a-c. 
Hoeglundina eocenica $($ Cushman and Hanna $)=$ Epistomina eocenica Cushman and Hanna, 1927, San Diego Soc. Nat. Hist., Trans, v. 5, p. 53, pl. 5, figs. 4-5. Ecology: upper bathyal biofacies, 150$500 \mathrm{~m}$ (Ingle, 1980).

Hormosina ovulum (Grzybowski) = Reophax ovulum Grzybowski, 1896, Akad. Umiejet. Krakowie, ser. V., 2, v. 30, p. 276, pl. 8, figs. 8, 9 .

Hyperammina elongata Brady-Mallory, 1959, p. 106, pl. 1, fig. 8; pl. 27, fig. 2.

Karreriella chapapotensis $($ Cole $)=$ Textularia chapatoensis Cole, 1928, Bull. Amer. Paleo., v. 14, p. 206, pl. 33, fig. 9. Range: early Eocene (P6b) into the Oligocene (Mallory, 1959; Tjalsma and Lohmann, 1983). Ecology: upper middle bathyal biofacies, 500-1500 m (Ingle, 1980).

Karreriella conversa Grzybowski) -Kaminski and Gradstein, 2005, p. 468-472, pl. 116, figs. 1-11.

Range: Late Cretaceous (Maastrichtian Stage) through at least early Eocene (P8) with questionable occurrences as young as middle Eocene (P11) (Gradstein and others, 1988; Kaminiski and others, 1988).

Karreriella elongata Mallory, 1959, p. 106, pl. 1, fig. 8; pl. 27, fig. 2. Range: early Eocene (P8) through Oligocene (Mallory, 1959; Tjalsma and Lohmann, 1983; McDougall, 2008). Ecology: lower middle bathyal biofacies, 1,500-2,000 m (Ingle, 1980).

Karreriella horrida Mjatliuk-Kaminski and Gradstein, 2005, 2005, p. 473-475, pl. 117, figs. 1-11. Range: late Paleocene through early Oligocene (Gradstein and others, 1988).

Lagena acuticosta Reuss-Mallory, 1959, p. 174, pl. 14, fig. 1a,b; pl. 28, fig. 10a,b; pl. 41, fig. 8a,b. Lagena becki Sullivan, 1962, Univ. Calif. Pub. Geol. Sci., v. 37, p. 266, pl. 10, figs. 16a-b.

Lagena costata (Williamson)—Mallory, 1959, p. 175, pl. 14, fig. 3a,b; pl. 41, fig. 7a,b.

Lagena gracilis Williamson, 1848, Ann. Mag. Nat. Hist., ser. 2, v. 1, p. 13, pl. 1, fig. 5.

Lagena hexagona (Williamson)-Mallory, 1959, p. 175, pl. 14, fig. 7.

Lagena laevis $($ Montagu $)=$ Vermiculum laeve Montagu, 1803, Testacea Britannica or Natural history of

British shells, marine, land and fresh-water, including the most minute, J.S. Hollis, Romsey,

England, p. 524.

Lagena paucicosta Franke = Lagena amphora Reuss paucicosta Franke-Smith, 1957, p. 169, pl. 23, fig. 9.

Lagena striata (d'Orbigny) = Oolina striata d'Orbigny, 1839, Voyage dans l'Amerique Meriodionale;

Foraminiferes, v. 5, pt. 5, p. 21, pl. 5, fig. 12.

Lagena vulgaris Williamson, 1858, On the recent Foraminifera of Great Britian, R. Soc. Lond., p. 3, pl. 1, figs. 5-5a.

Lenticulina altolimbata $($ Gumbel $)=$ Robulina alto-limbata Gumbel-Mallory, 1959, p. 133, pl. 6, fig. 16; pl. 27, fig. 11.

Lenticulina arcuatostriata $($ Hantken $)=$ Cristellaria $($ Robulina $)$ arcuato-striata Hantken, 1868, Magyarh. Foldt. Tars., Munk., Pest, Magyarorszag, kot. 4, p. 93, pl. 2, figs. 30a-c.

Lenticulina caritae Bermudez, 1949, Cushman Lab. Foram. Res., Spec. Pub., no. 25, p. 122, pl. 7, figs. 35-36.

Lenticulina carolinianus $($ Cushman $)=$ Robulus arcuatostriatus carolinianus Cushman, 1933, Cushman Lab. Foram. Res., Contr., v. 9, p. 4, pl. 1, fig. 9.

Lenticulina chambersi $($ Garrett $)=$ Robulus chambersi Garrett, 1939, Jour. Paleo., v. 13, p. 576, pl. 65, figs. 8-9.

Lenticulina convergens $($ Bornemann $)=$ Cristellaria convergens Bornemann, 1855, Deutsch. Geol. Ges., Zeitschr., Berlin, Deutschland, Bd. 7, Heft. 2, p. 327, pl. 13, figs. 16-17.

Lenticulina cultrata (Montfort) $=$ Robulus cultrata Montfort, 1808, Conchyliologie systematique et classification methodique des coquilles, v. 1, p. 215, tf. 
Lenticulina gyroscalpum $($ Stache $)=$ Cristellaria gyroscalpum Stache, 1865, Novara Exped. 1857-1859, Wien Osterrich, Geol. Theil, Bd. 1, Abt. 2, p. 243, pl. 23, fig. 22.

Lenticulina inornata (d'Orbigny) = Robulus inornata (d'Orbigny)-Mallory, 1959, p. 137, pl. 7, fig. 15, pl. 40 , fig. 5.

Lenticulina limbosus (Reuss) = Robulus limbosus (Reuss)—Mallory, 1959, p. 138, pl. 6, fig. 14.

Lenticulina limbosus hockleyensis (Cushman and Applin $)=$ Robulus limbosa hockleyensis Cushman and Applin-Mallory, 1959, p. 139, pl. 6, fig. 15a,b.

Lenticulina midwayensis (Plummer) = Cristellaria midwayensis Plummer, 1926, Texas Univ. Bull. , no. 2644, p. 95, pl. 13, fig. 5.

Lenticulina pseudocultratus $($ Cole $)=$ Robulus pseudocultratus Cole-Mallory, 1959, p. 140, pl. 7, fig. 10; pl. 27, fig. 10.

Lenticulina pseudomammiligera $($ Plummer $)=$ Cristellaria pseudo-mammiligera Plummer, 1926, Texas Univ. Bull., no. 2644, p. 98, pl. 7, fig. 11.

Lenticulina pseudovortex $($ Cole $)=$ Robulus pseudovortex Cole-Mallory, 1959, p. 141, pl. 7, figs. 2-3; pl. 27 , fig. 13.

Lenticulina rosettus $($ Gumbel $)=$ Rosalina rosetta Gumbel, 1870, K. Bayer Akad. Wiss. Math.-Phys. Abt., Abh, K12, v. 10, p. 642, pl. 1, fig. 73.

Lenticulina rotulata Lamarck-Mallory, 1959, p. 146, pl. 8, fig. 15.

Lenticulina simplex (d'Orbigny) = Robulus simplex (d'Orbigny) Kleinpell, 1938, p. 203, pl. 8, fig. 1.

Range: late Oligocene, late Zemorrian Stage through middle Miocene, early Relizian Stage (Kleinpell, 1938).

Lenticulina smileyi $($ Kleinpell $)=$ Robulus smileyi Kleinpell, 1938, p. 202-203, pl. 15, fig. 14a-b. Range: middle Miocene, late Relizian through late Luisian Stages (Kleinpell, 1938). Ecology: upper middle bathyal biofacies, 500-1500 m (Ingle, 1980).

Lenticulina terryi $($ Coryell and Embich $)=$ Robulus terryi Coryell and Embich-Mallory, 1959, p. 141, pl. 6, fig. 1.

Lenticulina theta Cole, 1927, Bull. Am. Paleo., v. 14, p. 14, pl. 1, fig. 17.

Lenticulina turbinatus (Plummer) = Cristellaria turbinatus Plummer, 1926, Texas Univ. Bull., no. 2644, p. 93, pl. 7, fig. 4.

Lenticulina ulatisensis $($ Boyd) $=$ Robulus ulatisensis Boyd in Mallory, 1959, p. 142, pl. 6, fig. 10a,b; pl. 40, fig. 4a,b. Range: late Penutian through early Narizian Stages (P9-P12) (Mallory, 1959; McDougall, 2008).

Lenticulina vortex $($ Fitchel and Moll $)=$ Robulus vortex (Fitchel and Moll)-Mallory, 1959, p. 142, pl. 7, fig. 1a,b.

Lenticulina welchi $($ Church $)=$ Robulus welchi Church, 1931, Calif. Dept. Nat. Res., Div. Mines, v. 27 , pl. C, figs. 13, 14. Range: middle and late Eocene, Ulatisian through late Narizian Stages (?P9P10, P11-P15) (Mallory, 1959; McDougall, 2008).

Lenticulina williamsoni (Reuss) = Cristellaria williamsoni Reuss, 862, K. Akad. Wiss., Wien, Math.Nat. Cl., Sitzber, Wien, Osterreich, Bd. 44, Abth. 1, p. 327, pl. 6, fig. 4.

Lituotuba lituiformis (Brady)-Mallory, 1959, p. 109, pl. 1, fig. 17. Range: Cretaceous through at least P8 (Mallory, 1959; Kaminiski and others, 1988; McDougall, 2008).

Loxostomoides applinae (Plummer) = Bolivina applinae Plummer, 1926, Texas Univ. Bull., no. 2644, pl. 4, fig. 1. Range: early Paleocene (P1) through late early Eocene (P6b); doubtful occurrence in early Eocene Zones P7 through P9 (van Morkhoven and others, 1986). Ecology: neritic biofacies, $\leq 150 \mathrm{~m}$ (Ingle, 1980; van Morkhoven and others, 1986).

Loxostomum delicatulum (Cushman)—Smith, 1957, p. 176-177, pl. 25, figs. 4a-b. 
Marginulina beali $($ Cushman $)=$ Cristellaria beali Cushman, 1925, Cushman Lab. Foram. Res., Contr., v. 1, p. 24-25, pl. 4, figs. 6-13. Range: middle Miocene, Relizian and Luisian Stages (Kleinpell, 1938). Ecology: outer neritic biofacies, $50-150$ m (Ingle, 1980).

Marginulina exima Neugeboren, 1851, Siebenb. Ver. Naturw. Hermannstadt, Verh. Mitt. Ungarn jahrg 2 , no. 8, p. 129.

Marginulina glabra d'Orbigny, 1826, Ann. Sci. Nat., p. 259, modele no. 55.

Marginulina munda Cushman 1938, Cushman Lab. Foram. Res., Contr., v. 14, p. 34, pl. 5, figs. 11, 12. Marginulina subbullata Hantken-Mallory, 1959, p. 151, pl. 9, figs. 13-15. Range: late Ynezian through late Narizian Stages (Mallory, 1959).

Marssonella oxycona (Reuss)-Mallory, 1959, p. 124, pl. 4, fig. 8a,b. Range: early Ynezian to early Penutian Stages, with questionable occurrence in the early Ulatisian Stage (Mallory, 1959).

Martinottiella eocenica (Cushman and Bermudez)-Mallory, 1959, p. 128, pl. 5, fig. 8a,b. Range: Eocene, early Penutian through late Narizian Stages (Mallory, 1959). Ecology: upper middle bathyal biofacies (Ingle, 1980).

Neoeponides hillebrandti Fisher, 1969, Paleo., v. 12, p. 197. Range: Paleocene (P1-P5) (Tjalsma and Lohmann, 1983). Range: Paleocene (P1-P5) (Tjalsma and Lohmann, 1983; McDougall, 2008). Ecology: Wide bathymetric range with most abundant occurrences at abyssal depths during the early Paleocene and bathyal depths during the late Paleocene (Tjalsma and Lohmann, 1983).

Neoflabellina jarvisi $($ Cushman $)=$ Flabellina jarvisis Cushman, 1935, Cushman Lab. Foram. Res., Contr. , v. 11, no. 4, p. 85, pl. 13, figs. 7-8. Range: Late Cretaceous (Maastrichtian Stage) through late Paleocene (P5) (van Morkhoven and others, 1986). Ecology: outer neritic and upper bathyal biofacies (van Morkhoven and others, 1986).

Nodosarella atlantisae hispidula $($ Cushman $)=$ Ellipsonodosaria atlantisae hispidula CushmanMallory, 1959, p. 221, pl. 19, fig. 2.

Nodosarella constricta Cushman and Bermudez, 1937, Cushman Lab. Foram. Res., Contr., v. 13, p. 18, pl. 2, figs. 4-7.

Nodosaria affinis Reuss-Cushman, 1951, U.S. Geol. Survey, Prof. Paper 232, p. 23, pl. 7, figs. 3-6. Nodosaria deliciae Martin, 1943, Stanford Univ. Pub. Geol. Sci., v. 3, p. 17, pl. 6, fig. 3. Range:

Eocene, late Bulitian through late Penutian Stages with rare occurrences in the early Narizian Stage (Mallory, 1959).

Nodosaria gyrata Mallory, 1959, p. 170, pl. 13, fig. 18. Range: Eocene, early Bulitian through late Narizian Stages (Mallory, 1959).

Nodosaria latejugata Gumbel-Mallory, 1959, p. 171, pl. 13, fig. 20; pl. 28, fig. 8; pl. 41, fig. 1. Range: Paleocene through middle Eocene, early Ynezian through late Ulatisian Stages (Mallory, 1959).

Nodosaria limbata d'Orbigny, 1840, Soc. Geol. France, Mem. Tome 4, no. 1, p. 12, pl. 1, fig. 1.

Nodosaria longiscata d'Orbigny, 1846, Foraminifers fossiles du bassin tertiaire de Vienna, (Autriche), Gide et Comp., Paris, France, p. 32, pl. 1, figs. 10, 12.

Nodosaria macneili Cushman-Mallory, 1959, p. 172, pl. 13, fig. 16. Range: late Bulitian Stage with rare occurrences in the late Ynezian Stage (Mallory, 1959).

Nodosaria pyrula d'Orbigny-Mallory, 1959, p. 172, pl. 13, fig. 19, pl. 41, fig. 2.

Nodosaria velascoensis Cushman-Mallory, 1959, p. 172, pl. 13, fig. 24. Range: Late Cretaceous

(Campanian Stage) through middle Eocene, early Narizian Stage (Mallory, 1959; Sliter, 1968). Nonion halkyardi Cushman, 1936, Cushman Lab. Foram. Res., Contr., v. 12, p. 63, pl. 12, fig. 1. Nonion inexcavatum $($ Cushman and Applin $)=$ Nonionina advena inexcavatum Cushman and Applin, 1926, Amer. Assoc. Pet. Geol., Bull., p. 182, pl. 10, figs. 18-19.

Nonionella ansata Cushman, 1946, U.S. Geol. Survey Prof. Paper, 206, p. 101, pl. 44, figs. 1a-c. 
Nonionella costifera $($ Cushman $)=$ Nonionina costifera Cushman, 1926, Cushman Lab. Foram. Res., Contr., v. 2, p. 90, pl. 13, figs. 2a-c. Range: Miocene to Holocene (Kleinpell, 1938; Finger, 1990). Ecology: inner neritic biofacies, $\leq 50 \mathrm{~m}$ (Ingle, 1980).

Nonionella jacksonensis Cushman, 1933, Cushman Lab. Foram. Res., Contr., v. 9, p. 10, pl. 1, fig. 23. Ecology: UDL is on the inner neritic biofacies, $\leq 50 \mathrm{~m}$ (Ingle, 1980).

Nonionella miocenica Cushman, 1926, Cushman Lab. Foram. Res., Contr., v. 1, p. 91, pl. 13, figs. 4a-c. Range: Oligocene to Holocene (Kleinpell, 1938; Finger, 1990). Ecology: inner neritic biofacies, $\leq 50 \mathrm{~m}$ (Ingle, 1980).

Nonionella montereyana Cushman and Galliher, 1934, Cushman Lab. Foram. Res., Contr., v. 10, p. 24, pl. 4, figs. 6a-b. Range: late Miocene, late Mohnian through early Delmontian Stages (Kleinpell, 1938). Ecology: outer neritic biofacies, 50-150 m (Ingle, 1980).

Nonionella multicamerata $($ Cushman and Kleinpell $)=$ Nonion pizarrensis Berry multicameratum Cushman and Kleinpell, 1934, Cushman Lab. Foram Res., Contr., v. 10, p. 4, pl. 1, figs. 10a-b. Range: late Miocene, Mohnian Stage (Kleinpell, 1938) and questionably in the Luisian Stage (Finger, 1990). Ecology: outer neritic biofacies, 50-150 m (Ingle, 1980).

Nonionella ovata Brotzen, 1948, Sver. Geol. Unders., Auh., Ser. C, no. 493, p. 68, pl. 10, figs. 15a-b.

Nonionella schencki Kleinpell, 1938,, p. 235-236, pl. 16, figs. 11a-b. Range: late Miocene, Mohnian through early Delmontian Stages (Kleinpell, 1938; Finger, 1990). Ecology: outer neritic biofacies, 50-150 m (Ingle, 1980).

Nuttaloides truempyi (Nuttall)= Eponides truempyi Nuttall, 1930, Jour. Paleo., v. 4, p. 287, pl. 24, figs. 9, 13, 14. Range: Late Cretaceous (Campanian Stage) through late Eocene (P17) (Mallory, 1959; van Morkhoven and others, 1986). Ecology: lower bathyal biofacies, 1,500-2,000 m (Ingle, 1980).

Oridorsalis tenera (Brady)_Kleinpell, 1938, p. 321-322.

Oridorsalis umbonatus (Reuss) -Finger, 1990, p. 48, pl. 9, figs. 16-18. Range: late Ynezian through late Narizian Stages (Mallory, 1959). Ecology: upper bathyal biofacies in the Paleogene and upper middle bathyal biofacies in the Neogene (Ingle, 1980).

Orthomorphina rohri (Cushman and Stainforth) = Nodogenerina rohri Cushman and Stainforth, 1945, Cushman Lab. Foram. Res., Spec. Pub., no. 14, pl. 39, pl. 5, fig. 26.

Osangularia mexicana $($ Cole $)=$ Pulvinulinella culter mexicana Cole, 1927, Bull. American Paleo., v. 14, p. 31, pl. 1, figs. 15-16. Range: early Eocene (P6b) into the Oligocene (Mallory, 1959; Tjalsma and Lohmann, 1983). Ecology: upper middle bathyal biofacies, 500-1500 m (Ingle, 1980).

Osangularia midwayana $($ Cushman and Todd $)=$ Truncatulina culter (Parker and Jones) midwayana Cushman and Todd, 1946, Cushman Lab. Foram. Res., Contr., v. 22, p. 63, pl. 11, fig. 12. Ecology: upper middle bathyal biofacies, 500-1500 m (Ingle, 1980).

Osangularia velascoensis $($ Cushman $)=$ Truncatulina velascoensis Cushman, 1925, Cushman Lab. Foram. Res., Contr., v. 1, p. 20, pl. 3, fig. 2. Range: Paleocene (P1c - P5) (van Morkhoven and others, 1986). Ecology: bathyal and abyssal species (van Morkhoven and others, 1986).

Palmula primitiva Cushman, 1939, Cushman Lab. Foram. Res., Contr., v. 15, p. 91, pl. 16, figs. 4-5. Range: Late Cretaceous through Paleocene (Cretaceous-P6a) (Mallory, 1959; Sliter, 1968; McDougall, 2008).

Planulina truncana (Gumbel)-Mallory, 1959, p. 148, pl. 9, fig. 8; pl. 27, fig. 17.

Plectina garzaensis Cushman and Siegfus, 1935, Cushman Lab. Foram. Res., Contr., v. 11, p. 92, pl. 14, figs. 3-4. Range: Eocene, early Ulatisian through late Narizian Stages with rare occurrences in the Penutian Stage (Mallory, 1959). 
Plectofrondicularia advena $($ Cushman $)=$ Frondicularia advena Cushman, 1923, U.S. Nat. Mus., Bull, no. 104, p. 141, pl. 20. figs. 1-2. Range: middle Miocene, Relizian Stage through Holocene (Kleinpell, 1938; Finger, 1990). Ecology: lower middle bathyal biofacies, 1,500-2,000 m (Cushman, 1922; Ingle, 1980).

Plectofrondicularia miocenica Cushman, 1926, Cushman Lab. Foram. Res., Contr., v. 2, p. 58, pl. 7,

figs. 10, 11; pl. 8, figs. 11, 12. Range: late Eocene, Refugian Stage through late Miocene,

Delmontian Stage (P17-N17) (Kleinpell, 1938; van Morkhoven and others, 1986; Finger, 1990;

McDougall, 2008). Ecology: lower middle bathyal biofacies, 1,500-2,000 m (Ingle, 1980).

Plectofrondicularia miocenica directa Cushman and Laiming, 1931, Jour. Paleo., v. 5, p. 105, pl. 11,

fig. 12. Range: early and middle Miocene, late Saucesian through early Relizian Stages

(Kleinpell, 1938, 1980).

Plectofrondicularia paucicostata Cushman and Jarvis, 1929, Cushman Lab. Foram. Res., Contr., v. 5, p.

10, pl. 2, figs. 11-12, 13. Range: middle Eocene (P12) through early Oligocene (P20) with

doubtful occurrence from early Eocene (P8) through middle Eocene (P11) (Mallory, 1959; van

Morkhoven and others, 1986). Ecology: middle bathyal to abyssal species (van Morkhoven and others, 1986).

Plectofrondicularia vaughani Cushman, 1927, Cushman Lab. Foram. Res., Contr., v. 3, pt. 2,, no. 41, p.

112, pl. 23, fig. 3. Range: early Oligocene (P19) through late Miocene (N17) (van Morkhoven

and others, 1986). Ecology: outer neritic biofacies, 50-150 m (van Morkhoven and others,

1986).

Pleurostomella acuta Hantken-Mallory, 1959, p. 218, pl. 29, fig 9a,b; pl. 37, fig. 7a-c. Range: late

Ynezian through late Narizian Stages (Mallory, 1959). Ecology: lower bathyal biofacies, 1,500-

2,000 m (Ingle, 1980).

Pleurostomella gredalensis Cook in Mallory, 1959, p. 218, pl. 18, fig. 15; pl. 35, fig. 3a,b.

Pleurostomella nuttalli Cushman and Siegfus, 1939, Cushman Lab. Foram. Res., Contr., v. 15, p. 29 , pl.

6, figs. 17, 18. Range: early Bulitian through early Narizian Stages (Mallory, 1959). Ecology:

lower bathyal biofacies, 1,500-2,000 m (Ingle, 1980).

Praeglobobulimina affinis (d'Orbigny) = Bulimina affinis d'Orbigny, 1839, Voyages dans l'Amerique

Meriodionale; Foraminiferes, v. 5, p. 105, pl. 2, figs. 25, 26. Ecology: upper middle bathyal biofacies, 150-500 m (Ingle, 1980).

Praeglobobulimina ovata (d'Orbigny) = Bulimina ovata d'Orbigny-Mallory, 1959, p. 195, pl. 16, fig.

4. Range: early Bulitian through late Narizian Stages (Mallory, 1959). Ecology: upper bathyal

biofacies, 150-500 m (Ingle, 1980).

Praeglobobulimina ovula (d'Orbigny) = Bulimina ovula d'Orbigny, 1838, Voyage dans l'Amerique

Meridonali, Foraminiferes, tome 5, p. 51, pl. 1, figs. 10-11. Range: Miocene, late Saucesian

through early Delmontian Stages with questionable occurrences in the Oligocene, Zemorrian

Stage (Kleinpell, 1938).

Praeglobobulimina pupoides (d'Orbigny) = Bulimina pupoides d'Orbigny-Mallory, 1959, p. 195, pl.

28, fig. 16a-c; pl. 36, fig. 17a-c. Range: early Penutian through late Narizian Stages (Mallory,

1959). Ecology: upper bathyal biofacies, 150-500 m (Ingle, 1980).

Praeglobobulimina pyrula (d'Orbigny) = Bulimina pyrula d'Orbigny, 1846, Foraminifers fossiles du

bassin tertiaire de Vienna, (Autriche), Gide et Comp., Paris, France, p. 184, pl. 11, figs. 9, 10.

Pseudoglandulina manifesta (Reuss)-Mallory, 1959, p. 174, pl. 13, fig. 26.

Pseudonodosaria conica (Neugeboren)-Mallory, 1959, p. 173, pl. 33, fig. 4, pl. 36, fig. 11a,b. Range:

late Ynezian through late Narizian Stages (Mallory, 1959). 
Pseudonodosaria inflata $($ Bornemann $)=$ Glandulina inflata Bornemann, 1855, Deutsch. Geol. Ges., Zeitschr., Berlin, v. 7, p. 320, pl. 12, figs. 6-7.

Pullenia eocenica Cushman and Siegfus, 1939, Cushman Lab. Foram. Res., Contr., v. 15, p. 31, pl. 7 , fig. 1a,b. Range: early Eocene (P9) through late Eocene (P17) with rare occurrences in the late Paleocene (P6a) (Tjalsma and Lohmann, 1983). Ecology: middle bathyal biofacies, 500-1500 m (Ingle, 1980).

Pullenia jarvis Cushman, 1936, Cushman Lab. Foram. Res., Spec. Pub., no. 6, p. 77, pl. 13, figs. 6a-b. Pullenia malkinae Coryell and Mossman, 1942, Jour. Paleo., v. 16, p. 234, pl. 36, figs. 3-4.

Pullenia miocenica Kleinpell, 1938, p. 338, pl. 14, fig. 6. Range: Oligocene to Miocene (Finger, 1980); most abundant in the middle Miocene (Relizian and Luisian Stages) of California (Kleinpell, 1938). Ecology: transitional between upper middle bathyal and lower middle bathyal biofacies (Ingle, 1980).

Pullenia quatriloba $($ Sequenza $)=$ Nonionina quatriloba Sequenza, 1880, R. Accad. Lincei, Rome, Cl. Sci. Fis., Mat., Nat., Riem., Roma, Italia, ser. 3, v. 6, p. 430, pl. 17, fig. 15.

Pullenia quinqueloba (Reuss)-Mallory, 1959, p. 246, pl. 34, fig. 1a,b. Range: late Ynezian through early Narizian Stage (Mallory, 1959). Ecology: upper middle bathyal biofacies, 500-1500 m (Ingle, 1980).

Pullenia salisburyi Stewart and Stewart, 1930, Jour. Paleo., v. 4, p. 72, pl. 8, fig. 2. Range: late Eocene, Narizian Stage into younger strata (Mallory, 1959; McDougall, 1980). Ecology: outer neritic biofacies, 50-150 m (Ingle, 1980).

Pyrulina cylindroides (Roemer) = Polymorphina cylindroides Roemer, 1853, Neues Jahrb. Min. Geogn. Geol. Petref.-Kund, p. 385, pl. 3, fig. 26a,b.

Quadrimorphina allomorphinoides (Reuss)—Mallory, 1959, p. 245, pl. 22, fig. 5a-c; pl. 34, fig. 2 a-c. Quinqueloculina josephina d'Orbigny, 1846, Foraminifers fossiles du bassin tertiaire de Vienna,

(Autriche), Gide et Comp., Paris, France, p. 297, pl. 19, figs. 25-27.

Quinqueloculina triangularis d'Orbigny-Smith, 1957, p. 155, pl. 19, fig. 7. Range: late Ulatisian Stage (Mallory, 1959). Ecology: inner neritic biofacies, $\leq 50 \mathrm{~m}$ (Ingle, 1980).

Reophax pilulifera Brady, 1883 Rep. Scientific Results Explor. Voyage HMS Challenger, Zool., v. 9, p. 292, pl. 30, figs. 18-20. Ecology: Bathyal to abyssal areas with coarse substrate (Kaminiski and Gradstein, 2005).

Reophax subfusiformis Earland, 1933, Foraminifera; Part II - South Georgia, Discovery Reports, Cambridge, England, v. 7, p. 74, pl. 2, figs. 16-19.

Rhabdammina eocenica Cushman and Hanna, 1927, Calif. Acad. Sci. Proc., 4th ser., v. 16, p. 209 , pl. 13, fig. 3. Ecology: upper middle bathyal biofacies, 1500-2000 m (Ingle, 1980).

Rosalina columbiensis $($ Cushman $)=$ Discorbis columbiensis Cushman, 1925, Cushman Lab. Foram .

Res., Contr., v. 1, p. 43, pl. 6, fig. 13. Ecology: inner neritic biofacies, $\leq 50 \mathrm{~m}$ (Ingle and Keller, 1980).

Saccammina complanata $($ Franke $)=$ Pelosina complanata Franke, 1912, K. Preuss. Geol. Lande Sanst. Bergakad., Berlin, v. 32, pl. 3, figs. 1a-b.

Saracenaria triangularis (d'Orbigny)—Smith, 1957 , p. 168, pl. 23, fig. 3.

Sigmoilina tenuis (Czjzek) = Quinqueloculina tenuis Czjzek, 1848, Haidinger's Nat. Wiss., Abh. 2, p. 149, pl. 13, figs. 31-34. Ecology: upper middle bathyal biofacies, 500-1500 m (Ingle, 1980).

Silicosigmoilina californica Cushman and Church, 1929, Calif. Acad. Sci. Proc., 4th ser., v. 18, p. 502, pl. 36, figs. 10-12. Range: Late Cretaceous (Campanian) through early Eocene (P9) with doubtful occurrences in middle Eocene zones P10 through P13 (Mallory, 1959; van Morkhoven 
and others, 1986; Almgren and others, 1988; McDougall, 2008). Ecology: common in bathyal and abyssal environments (van Morkhoven and others, 1986).

Siphogenerina branneri $($ Bagg) = Sagrina branneri Bagg, 1905, U.S. Geological Survey, Bull. 268, p. 40, pl. 7, fig. 4. Range: early and middle Miocene, late Saucesian through Luisian Stages and questionably in the Mohnian Stage (Kleinpell, 1938; Finger, 1990). Ecology: upper middle bathyal biofacies, 500-1500 m (Ingle, 1980).

Siphogenerina collomi Cushman, 1925, Cushman Lab. Foram. Res., Contr. , v. 1, p. 2, pl. 4, fig. 3. Range: middle Miocene, late Luisian Stage (Kleinpell, 1938).

Siphogenerina hughesi Cushman, 1925, Cushman Lab. Foram. Res., Contr. , v. 1, p. 36, pl. 7, fig. 4. Range: middle Miocene, Relizian through Luisian Stages (Kleinpell, 1938; Finger, 1990). Ecology: upper middle bathyal biofacies, 500-1500 m (Ingle, 1980).

Siphogenerina transversa Cushman = Siphogenerina raphanus transversus Cushman, 1918, U.S. Nat. Mus., Bull, no. 103, p. 64, pl. 22, fig. 8. Range: late Oligocene through middle Miocene, Zemorrian through late Saucesian Stages (N4-N11) (Kleinpell, 1938; van Morkhoven and others, 1986). Ecology: upper bathyal biofacies, 150-500 m (Ingle, 1980; van Morkhoven and others, 1986).

Siphonia jacksonensis Cushman and Applin, 1926, Amer. Assoc. Petr. Geol., Bull., v. 10, p. 180, pl. 9, figs. 20-23.

Siphonia wilcoxensis Cushman, 1927, U.S. Nat. Mus. Proc., v. 72, art. 20, p. 3, pl. 2, figs. 1-3. Range: early Ynezian to late Penutian Stages (Mallory, 1959).

Siphonodosaria gracillima (Cushman and Jarvis) = Ellipsonodosaria nuttalli Cushman and Jarvis gracillima Cushman and Jarvis, 1934, Cushman Lab. Foram. Res., Contr., v. 10, p. 72, pl. 10, fig. 7. Range: early Eocene (P9) to late Eocene (P15) and it is particularly abundant in P11 (Tjalsma and Lohmann, 1983. Ecology: upper middle bathyal biofacies, 500-1500 m (Tjalsma and Lohmann, 1983).

Spiroloculina lamposa Hussey, 1949, Jour. Paleo., v. 23, p. 121, pl. 26, fig. 6.

Spiroloculina texana Cushman and Ellisor, 1944, Cushman Lab. Foram. Res., Contr., v. 20, p. 51, pl. 8, figs. 14-15.

Spiroplectammina directa Cushman and Siegfus, 1939, Cushman Lab. Foram. Res., Contr., v. 15, p. 26, pl. 6, figs. 7-8. Range: Late Cretaceous through late middle Eocene (P14) (Gradstein and others, 1988; Kaminiski and others, 1988; McDougall, 2008). Ecology: lower upper bathyal biofacies in the Paleocene and in the upper part of the upper bathyal biofacies in the Eocene (van Morkhoven and others, 1986).

Spiroplectammina richardi Martin, 1943, Stanford Univ. Pub. Geol. Sci., v. 3, p. 14, pl. 5, fig. 3a,b. Range: late Paleocene, Ynezian Stage, through the middle Eocene, early Narizian Stage (P4P14) (Mallory, 1959; Almgren and others, 1988; McDougall, 2008). Ecology: outer neritic biofacies, 50-150 m (Ingle, 1980).

Spiroplectammina tejonensis Mallory, 1959, p. 118, pl. 3, fig. 6-8. Range: late Ulatisian through late Narizian Stages (Mallory, 1959).

Stensioina beccariformis $($ White $)=$ Rotalia beccariiformis White, 1928, Jour. Paleo., v. 2, p. 287, pl. 39, figs. 2-4. Range: Late Cretaceous (Santonian Stage) through Paleocene (P5) (van Morkhoven and others, 1986; McDougall, 2008). Ecology: bathyal and abyssal species (van Morkhoven and others, 1986).

Stichocassidulina thalmani Stone, 1946, Jour. Paleo, v. 20, p. 59-60, tfs. 1-2. Range; late Eocene, Narizian and Refugian Stages (Mallory, 1959; McDougall, 1980). 
Stilostomella adolphina (d'Orbigny) = Nodogenerina adolphina (d'Orbigny)—Mallory, 1959, p. 216, pl. 18, fig. 8; pl. 41, fig. 10. Ecology: lower middle bathyal biofacies, 1,500-2,000m (Ingle, 1980).

Stilostomella advena $($ Cushman and Siegfus) $=$ Nodosarella advena Cushman and Siegfus, 1939, Cushman Lab. Foram. Res., Contr., v. 15, p. 30, pl. 6, figs. 19, 20. Range: early Eocene, late Penutian Stage, through middle Eocene, early Narizian Stage (Mallory, 1959; McDougall, unpublished). Ecology: lower middle bathyal biofacies, 1,500-2,000 m (Ingle, 1980).

Stilostomella gracilis $($ Palmer and Bermudez $)=$ Ellipsonodosaria gracilis Palmer and Bermudez, 1936, Soc. Cubana Hist. Nat. Mem., v. 10, p. 296, pl. 18, figs. 18-19.

Stilostomella lepidula $($ Schwager $)=$ Nodogenerina lepidula (Schwager)-Mallory, 1959, p. 217, pl. 18, fig. 10. Ecology: lower middle bathyal biofacies, 1,500-2,000 m (Ingle, 1980).

Stilostomella paleocenica $($ Cushman and Todd $)=$ Nodosarella paleocenica Cushman and Todd, 1946, Cushman Lab. Foram. Res., Contr., v. 22, p. 60, pl. 10, fig. 23.

Tappanina selmensis (Cushman)—van Morkhoven and others, 1986, p. 332-334, pl. 108, fig. 1-3. Range: Late Cretaceous (Maastrichtian Stage) through early Eocene (P6b) (van Morkhoven and others, 1986). Ecology: outer neritic biofacies, 50-150 m (van Morkhoven and others, 1986).

Textularia adalta Cushman, 1926, Cushman Lab. Foram. Res., Contr., v. 2, p. 29, pl. 4, fig. 2. Range: early Ynezian through early Narizian Stages (Mallory, 1959).

Textularia conica d'Orbigny, 1939, in de La Sagra, Hist. Phys., Pol., Nat. Cuba, "Foraminiferes", p. 143, v. 8, pl. 1, figs. 19, 20. Ecology: inner neritic biofacies, $\leq 50 \mathrm{~m}$ (Bandy, 1961).

Textularia plummerae Lalicker, 1935, Cushman Lab. Foram. Res., Contr., v. 11, p. 50, pl. 6, fig. 10. Trifarina advena californica Mallory, 1959, p. 210-211, pl. 17, fig. 14a-d; pl. 29, fig. 6a-c; pl. 40, fig. 11a,b. Range: Bulitian through Ulatisian Stages (Mallory, 1959). Ecology: transitional between the outer neritic and the upper bathyal biofacies (Ingle, 1980).

Trifarina angulosa (Williamson) —Lankford and Phleger, 1973, Jour. Foram. Res., v. 3, p. 129, pl. 3, figs. 29, 30. Ecology: transitional between the outer neritic and upper bathyal biofacies (Ingle, 1980).

Trifarina wilcoxensis (Cushman and Ponton) $=$ Pseudouvigerina wilcoxensis Cushman and Ponton, 1932, Cushman Lab. Foram. Res., Contr., v. 8, p. 66, pl. 8, fig. 18. Range: early through middle Eocene, Penutian and Ulatisian Stages (P6b-P10) (Mallory, 1959; Almgren and others, 1988; McDougall, 2008). Ecology: outer neritic biofacies; transitional to upper bathyal biofacies (Ingle, 1980).

Tritaxilina colei Cushman and Siegfus, 1935, Cushman Lab. Foram. Res., Contr., v. 11, p. 92, pl. 14, figs. 5-6. Range: late Ynezian through late Narizian Stages (Mallory, 1959).

Tritaxia globulifera $($ ten Dam and Sigal $)=$ Pseudoclavulina globulifera ten Dam and Sigal, 1950, Cushman Found. Foram. Res., Contr., v. 1, p. 32, pl. 2, figs. 5-7. Range: Paleocene (P1 P6a)(Kaminiski and others, 1988). Ecology: lower middle bathyal biofacies, 1,500-2,000 m (Tjalsma and Lohmann, 1983; Kaminiski and others, 1988).

Trochammina globigeriniformis = Lituola globigeriniformis Parker and Jones, 1865, R. Soc. London Philos. Trans., v. 155, p. 407, pl. 15, figs. 46-47.

Trochamminoides conglobatulus $($ Brady) $=$ Trochammina conglobata Brady, 1884, Rept. Challenger Exped., England, Zool., pt. 22, v. 9, p. 341, pl. 40, figs. 8-9.

Trochamminoides contortus Mallory, 1959, p. 110, pl. 2, fig. 1a,b. Range: Eocene, late Bulitian through early Narizian Stages with questionable occurrences in the Paleocene, late Ynezian Stage (Mallory, 1959). 
Uvigerina alabamensis Cushman and Garrett, 1939, Cushman Lab. Foram. Res., Contr., p. 83, pl. 14, figs. 26, 27. Range: Penutian through early Narizian Stages (Mallory, 1959).

Uvigerina beccarii Fornasini-Kleinpell, 1938, p. 293, pl. 5, figs. 3, 4. Range: Oligocene and early Miocene, early Zemorrian through early Saucesian Stages (Kleinpell, 1938).

Uvigerina churchi Cushman and Siegfus, 1939, Cushman Lab. Foram. Res., Contr., v. 15, p. 29, pl. 6, fig. 16. Range: middle and late Eocene, Ulatisian and Narizian Stages (P10-P15)(Mallory, 1959; McDougall, 2008).

Uvigerina cocoaensis Cushman, 1925, Cushman Lab. Foram. Res., Contr., v. 1, p. 68, pl. 10, fig. 12. Range: middle Eocene through Oligocene (P9-P22) (Boersma, 1984), however documented occurrences in California are limited to the Refugian (P15-P17) (McDougall, 2008). Ecology: upper bathyal biofacies, 150-500 m (Boersma, 1984).

Uvigerina elongata Cole, 1927, Bull. Amer. Paleo., v. 14, p. 11, pl. 4, figs. 2-3. Range: middle Eocene (P9) through early Oligocene (P20) (Boersma, 1984). Ecology: outer neritic biofacies, 150-500 m (Boersma, 1984).

Uvigerina gardnerae Cushman, 1926, Amer. Assoc. Petr. Geol. Bull., v. 10, p. 175,pl. 8, figs. 16-17. Uvigerina garzaensis Cushman and Siegfus, 1939, Cushman Lab. Foram. Res., Contr., v. 15, p. 28 , pl. 6, fig. 15a,b. Range: middle through late Eocene, Ulatisian through Refugian Stages (P10-P17) (Mallory, 1959; McDougall, 2008). Ecology: lower bathyal biofacies, 1,500-2,000 m (Ingle, 1980).

Uvigerina gesteri Barbat and von Estorff, 1933, Jour. Paleo., v. 7, p. 171, pl. 23, figs. 7, 18. Range: restricted to the lower Zemorrian Stages (Kleinpell, 1980).

Uvigerina hispida Schwager — von Morkhoven and others, 1986, p. 62-64, pl. 20, figs. 1-4. Range: middle Miocene (N5) to Holocene (Boersma, 1984; van Morkhoven and others, 1986). Ecology: lower middle bathyal biofacies, 1,500-2,000 m (Ingle, 1980; Ingle and Keller, 1980).

Uvigerina joaquinensis Kleinpell, 1938, p. 296, pl. 17, figs. 6, 10, 11. Range: middle Miocene, late Luisian Stage (Kleinpell, 1938).

Uvigerina laimingi Smith, 1957, p. 177-178, pl. 26, fig. 4a-c, 8a-c. Range: late Paleocene, Ynezian Stage (P4)(Mallory, 1959; McDougall, unpublished).

Uvigerina lodoensis miriamae Mallory, 1959, p. 209, pl. 17, figs. 8-9; not pl. 40, fig. 9. Range: early Eocene, late Penutian through early Ulatisian Stages (P6b-P9, ?P10)(Mallory, 1959; McDougall, 2008). Ecology: shallow shelf depths, frequently in warmer regions and associated with glauconite (Boersma, 1984).

Uvigerina rustica Cushman and Edwards, 1938, Cushman Lab. Foram. Res., Contr., v. 14, p. 83, pl. 14, fig. 6. Range: late Eocene through the Miocene (P15-N17) (Boersma, 1984). Ecology: lower middle bathyal biofacies, 1,500-2,000 m (Pflum and Frerichs, 1976).

Uvigerina subperegrina Cushman and Kleinpell, 1934, Cushman Lab. Foram. Res., Contr., v. 10, p. 12, pl. 2, figs. 9-11. Range: late Miocene, Mohnian through late Delmontian stages (Kleinpell, 1938). Ecology: upper bathyal biofacies, 150-500 m (Ingle, 1980); low oxygen indicator (Blake, 1991).

Uvigerinella californica Cushman, 1926, Cushman Lab. Foram. Res., Contr., v. 2, p. 58, pl. 8, figs. 2ab, 5. Range: late Oligocene, late Zemorrian Stage through middle Miocene, Luisian Stage (Kleinpell, 1938). Ecology: upper bathyal biofacies, 150-500 m (Ingle, 1980).

Uvigerinella obesa Cushman, 1926, Cushman Lab. Foram. Res., Contr., v. 2, p. 59, pl. 8, figs. $3 a-c, 7$. Range: early and middle Miocene, Saucesian through Relizian stages (Kleinpell, 1938). 
Uvigerinella obesa impolita Cushman and Laiming, 1931, Jour. Paleo., v. 5, p. 11, pl. 12, fig. 11a,b; p. 88. Range: Oligocene, Zemorrian Stage through early Miocene, Saucesian Stage (Kleinpell, 1938).

Vaginulinopsis asperuliformis $($ Nuttall $)=$ Cristellaria asperuliformis Nuttall, 1930, Jour. Paleo., v. 4, p. 282, pl. 23, figs. 9, 10. Range: early and middle Eocene, Penutian through early Narizian Stages (P6b-P14) (Mallory, 1959; Almgren and others, 1988; McDougall, 2008). Ecology: outer neritic biofacies, 50-150 m (Ingle, 1980).

Vaginulinopsis earlandi (Plummer) = Cristellaria earlandi Plummer, 1927, Texas Univ. Bull., no. 2644, p. 103, pl. 7, fig. 10.

Vaginulinopsis longiformis (Plummer) $=$ Cristellaria longiforma Plummer, 1927, Texas Univ. Bull., no. 2644, p. 102, pl. 13, fig. 4. Range: Paleocene (Cushman, 1951).

Vaginulinopsis nudicostata $($ Cushman and Hanna $)=$ Cristellaria mexicana nudicostata Cushman and Hanna, 1927, Calif. Acad. Sci., Proc., ser. 4, v. 16, p. 216, pl. 14, fig. 2.Range: middle Eocene, early Ulatisian through early Narizian Stages (P9-P10) (Mallory, 1959; Almgren and others, 1988; McDougall, 2008).

Vaginulinopsis saundersi $($ Hanna and Hanna $)=$ Cristellaria saundersi Hanna and Hanna, 1924, Univ . Washington Pub. Geol. Sci., v. 1, p. 61, pl. 13, figs. 5, 6, 15. Range: early Eocene, late Penutian through early Ulatisian Stages with questionable occurrences in the late Paleocene, Ynezian Stage and middle Eocene, Ulatisian Stage (?P4-P6, P7-P9, ?P10-P16)(Almgren and others, 1988; McDougall, 2008).

Vaginulinopsis tuberculata $($ Plummer $)=$ Cristellaria subaculeata tuberculata Plummer, 1927, Texas Univ. Bull, no. 2644, p. 101, pl. 7, fig. 2; pl. 14, fig. 1. Range: Paleocene (Cushman, 1951). Vaginulinopsis vacavillensis $($ Hanna) = Cristellaria vacavillensis Hanna, 1923, Univ. Calif. Pub. Geol. Sci., v. 14, no. 9, p. 324, pl. 59, fig. 4. Range: early and middle Eocene, Penutian through late Ulatisian Stages (P8-P10) (Mallory, 1959; Almgren and others, 1988; McDougall, 2008).

Vaginulinopsis verruculosa Martin - McDougall (2008). Range: early Eocene, Penutian Stage (P7-P9) (Mallory, 1959; Almgren and others, 1988; McDougall, 2008).

Valvulineria araucana (d'Orbigny)—Kleinpell, 1938, p. 307-308. Range: late Miocene, early Mohnian Stage to Holocene (Kleinpell, 1938). Ecology: upper middle bathyal biofacies, 500-1500 m (Ingle, 1980).

Valvulineria californica Cushman, 1925, Cushman Lab. Foram. Res., Contr. , v. 2, p. 60, pl. 9, figs. 1ac. Range: middle Miocene, Luisian Stage (Kleinpell, 1938; Finger, 1990). Ecology: upper middle bathyal biofacies, 500-1500 m (Ingle, 1980).

Valvulineria jacksonensis welcomensis Mallory, 1959, p. 231, pl. 20, figs. 3a-c, 5a-c. Range: $V$. jacksonensis welcomensis ranges from early Narizian through Refugian Stage (Mallory, 1959; McDougall, 1980).

Valvulineria martinezensis Smith, 1957, p. 180, pl. 27, figs. 1, 4.

Valvulineria miocenica Cushman, 1926, Cushman Lab. Foram. Res., Contr. , v. 2, p. 61, pl. 8, figs. 9, 10; pl. 9, figs. 3a-c. Range: middle Miocene, Luisian Stage (Kleinpell, 1938). Ecology: outer neritic biofacies, 50-150 m (Ingle, 1980).

Valvulineria tumeyensis Cushman and Simonson, 1944, Jour. Paleo., v. 18, p. 201, pl. 33, figs. $13,14$. Range: early Narizian through Refugian Stages (P13-P17) (Mallory, 1959; Donnelly, 1976; McDougall, 1980, 2008).

Valvulineria williami Kleinpell, 1938, p. 315, pl. 7, figs. 14a-c. Range: early and middle Miocene, late Saucesian to late Relizian Stages (Kleinpell, 1938). 
Valvulineria wilcoxensis Cushman and Ponton, 1932, Cushman Lab. Foram. Res., Contr. v. 8, p. 70, pl. 9, figs. 6a-c. Range: Valvulineria wilcoxensis ranges from the Bulitian through the early Ulatisian Stages with a questionable occurrence in the late Ynezian (Mallory, 1959).

Virgulinella pertusa (Reuss) = Virgulina pertusa Reuss, 1861, Beitrage sur Kenntniss der tertiaren Foraminiferen-Fauna. K. Akad. Wiss. Wien, Math.-Naturw. Cl., sitzber., Wien, Osterreich. Bd. 42, Heft 24 (Jahrg. 1860), p. 362, pl. 2, fig. 16. Range: late Oligocene and Miocene (Revets, 1991; McDougall, 1995).

Verneuilina triangulata Cook in Mallory, 1959, p. 120, pl. 4, fig. 1a-c; pl. 33, fig. 1a,b. Range: restricted to the early Penutian Stage (Mallory, 1959).

Vulvulina curta Cushman and Siegfus, 1935, Cushman Lab. Foram. Res., Contr., v. 11, p. 91, pl. 14, figs. 1, 2. Range: late Ynezian through late Narizian stages (Mallory, 1959). Ecology: lower middle bathyal biofacies, 1,500-2,000 m (Ingle, 1980). 